UNIVERSIDAD POLITÉCNICA DE MADRID

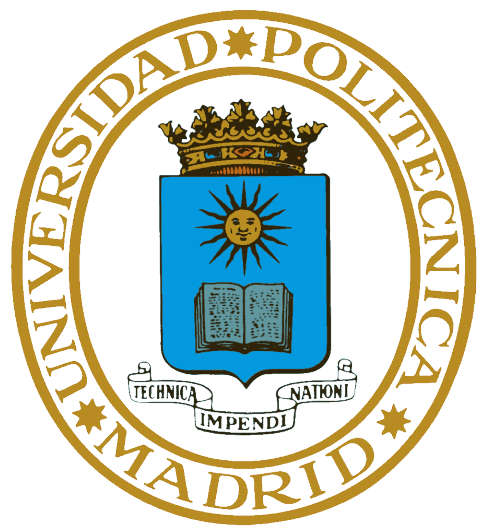

ESCUELA TÉCNICA SUPERIOR DE INGENIEROS INFORMÁTICOS

\title{
ON THE USE OF QUASIORDERS IN FORMAL LANGUAGE THEORY
}

\author{
PH.D THESIS
}

\section{Pedro Valero}

Double Degree in Computer Science and Mathematics 
Copyright $\odot 2020$ by Pedro Valero 
DEPARTAMENTAMENTO DE LENGUAJES Y SISTEMAS INFORMÁTICOS E INGENIERIA DE SOFTWARE

ESCUELA TÉCNICA SUPERIOR DE INGENIEROS INFORMÁTICOS

\section{ON THE USE OF QUASIORDERS IN FORMAL LANGUAGE THEORY}

SUBMITTED IN PARTIAL FULFILLMENT OF THE REQUIREMENTS FOR THE DEGREE OF:

Doctor of Philosophy in Software, Systems and Computing

Author: Pedro Valero

Double Degree in Computer Science and Mathematics

Advisor: Dr. Pierre Ganty

Ph.D. in Computer Science

May 2020

Thesis Committee:

Prof. Javier Esparza, Technische Universität München, Germany

Prof. Manuel Hermenegildo, Instituto IMDEA Software, Spain

Prof. Ricardo Peña, Universidad Complutense de Madrid, Spain

Prof. Samir Genaim, Universidad Complutense de Madrid, Spain

Prof. Parosh Aziz Abdulla, Uppsala Universitet, Sweden 



\section{Abstract of the Dissertation}

In this thesis we use quasiorders on words to offer a new perspective on two wellstudied problems from Formal Language Theory: deciding language inclusion and manipulating the finite automata representations of regular languages.

First, we present a generic quasiorder-based framework that, when instantiated with different quasiorders, yields different algorithms (some of them new) for deciding language inclusion. We then instantiate this framework to devise an efficient algorithm for searching with regular expressions on grammar-compressed text. Finally, define a framework of quasiorder-based automata constructions to offer a new perspective on residual automata.

\section{The Language Inclusion Problem}

First, we study the language inclusion problem $L_{1} \subseteq L_{2}$ where $L_{1}$ is regular or context-free and $L_{2}$ is regular. Our approach relies on checking whether an overapproximation of $L_{1}$, obtained by successively over-approximating the Kleene iterates of its least fixpoint characterization, is included in $L_{2}$. We show that a language inclusion problem is decidable whenever the over-approximating function satisfies a completeness condition (i.e. its loss of precision causes no false alarm) and prevents infinite ascending chains (i.e. it guarantees termination of least fixpoint computations).

Such over-approximation of $L_{1}$ can be defined using quasiorder relations on words where the over-approximation gives the language of all words "greater than or equal to" a given input word for that quasiorder. We put forward a range of quasiorders that allow us to systematically design decision procedures for dif- 
ferent language inclusion problems such as regular languages into regular languages or into trace sets of one-counter nets and context-free languages into regular languages.

Some of the obtained inclusion checking procedures correspond to wellknown algorithms like the so-called antichains algorithms. On the other hand, our quasiorder-based framework allows us to derive an equivalent greatest fixpoint language inclusion check which relies on quotients of languages and which, to the best of our knowledge, was not previously known.

\section{Searching on Compressed Text}

Secondly, we instantiate our quasiorder-based framework for the scenario in which $L_{1}$ consists on a single word generated by a context-free grammar and $L_{2}$ is the regular language generated by an automaton. The resulting algorithm can be used for deciding whether a grammar-compressed text contains a match for a regular expression.

We then extend this algorithm in order to count the number of lines in the uncompressed text that contain a match for the regular expression. We show that this extension runs in time linear in the size of the compressed data, which might be exponentially smaller than the uncompressed text.

Furthermore, we propose efficient data structures that yield optimal complexity bounds and an implementation -zearch-that outperforms the state of the art, offering up to $40 \%$ speedup with respect to highly optimized implementations of the decompress and search approach.

\section{Residual Finite-State Automata}

Finally, we present a framework of finite-state automata constructions based on quasiorders over words to provide new insights on residual finite-state automata (RFA for short).

We present a new residualization operation and show that the residual equivalent of the double-reversal method holds, i.e. our residualization operation applied to a co-residual automaton generating the language $L$ yields the canonical RFA for $L$. We then present a generalization of the double-reversal method for RFAs along the lines of the one for deterministic automata.

Moreover, we use our quasiorder-based framework to offer a new perspective on $\mathrm{NL}^{*}$, an on-line learning algorithm for RFAs.

We conclude that quasiorders are fundamental to residual automata in the same way congruences are fundamental for deterministic automata. 


\section{Resumen de la Tesis Doctoral}

En esta tesis, usamos preórdenes para dar un nuevo enfoque a dos problemas fundamentales en Teoría de Lenguajes Formales: decidir la inclusión entre lenguajes y manipular la representación de lenguajes regulares como autómatas finitos.

En primer lugar, presentamos un esquema que, dado un preorden que satisface ciertos requisitos, nos permite derivar de manera sistemática algoritmos de decisión para la inclusión entre diferentes tipos de lenguajes. Partiendo de este esquema desarrollamos un algoritmo de búsqueda con expresiones regulares en textos comprimidos mediante gramáticas. Por último, presentamos una serie de autómatas, cuya definición depende de un preorden, que nos permite ofrecer un nuevo enfoque sobre la clase de autómatas residuales.

\section{El Problema de la Inclusión de Lenguajes}

En primer lugar, estudiamos el problema de decidir $L_{1} \subseteq L_{2}$, donde $L_{1}$ es un lenguaje independiente de contexto y $L_{2}$ es un lenguaje regular. Para resolver este problema, sobre-aproximamos los sucesivos pasos de la iteración de punto fijo que define el lenguaje $L_{1}$. Con ello, obtenemos una sobre-aproximación de $L_{1} \mathrm{y}$ comprobamos si está incluida en el lenguaje $L_{2}$. Esta técnica funciona siempre y cuando la sobre-aproximación sea completa (es decir, la imprecisión de la aproximación no produzca falsas alarmas) y evite cadenas infinitas ascendentes (es decir, garantice que la iteración de punto fijo termina).

Para definir una sobre-aproximación que cumple estas condiciones, usamos un preorden. De este modo, la aproximación del lenguaje $L_{1}$ contiene todas las palabras "mayores o iguales que" alguna palabra de $L_{1}$. En concreto, definimos 
una serie de preórdenes que nos permiten derivar, de manera sistemática, algoritmos de decisión para diferentes problemas de inclusión de lenguajes como la inclusión entre lenguajes regulares o la inclusión de lenguajes independientes de contexto en lenguajes regulares.

Algunos de los algoritmos obtenidos mediante esta técnica coinciden con algoritmos bien conocidos como los llamados antichains algorithms. Por otro lado, nuestra técnica también nos permite derivar algoritmos de punto fijo que, hasta donde sabemos, no han sido descritos anteriormente.

\section{Búsqueda en textos comprimidos}

En segundo lugar, aplicamos nuestro algoritmo de decisión de inclusión entre lenguajes al problema $L_{1} \subseteq L_{2}$, donde $L_{1}$ es un lenguaje descrito por una gramática que genera una única palabra y $L_{2}$ es un lenguaje regular definido por un autómata o expresión regular. De esta manera, obtenemos un algoritmo que nos permite decidir si un texto comprimido mediante una gramática contiene, o no, una coincidencia de una expresión regular dada.

Posteriormente, modificamos este algoritmo para contar las líneas del texto comprimido que contienen coincidencias de la expresión regular. De este modo, obtenemos un algoritmo que opera en tiempo linear respecto del tamaño del texto comprimido el cual, por definición, puede ser exponencialmente más pequeño que el texto original.

Además, describimos las estructuras de datos necesarias para que nuestro algoritmo opere en tiempo óptimo y presentamos una implementación -zearchque resulta hasta un $40 \%$ más rápida que las las mejores implementaciones del método estándar de descompresión y búsqueda.

\section{Autómatas Residuales}

Finalmente presentamos una serie de autómatas parametrizados por preórdenes que nos permiten mejorar nuestra compresión de la clase de autómatas residuales (abreviados como RFA).

Estos autómatas parametrizados nos permiten definir una nueva operación de residualization y demostrar que el método de double-reversal funciona para RFAs, es decir, residualizar un autómata cuyo reverso es residual da lugar al canonical RFA (un RFA de tamaño mínimo). Tras esto, generalizamos este método de forma similar a su generalización para el caso de autómatas deterministas. Por último, damos un nuevo enfoque a $\mathrm{NL}^{*}$, un algoritmo de aprendizaje de RFAs.

Como conclusión, encontramos que los preórdenes juegan el mismo papel para los autómatas residuales que las congruencias para los deterministas. 
To my parents and my wife, for their endless love and support 



\section{ACKNOWLEDGMents}

Tras un proyecto tan largo e intenso como un doctorado, la lista de personas a las que quiero dar las gracias es muy extensa. En general, quiero dar las gracias a todas aquellas personas que, de un modo $\mathrm{u}$ otro, han formado parte de mi vida durante estos últimos años. En las siguientes lineas trataré de nombrarlos a todos, aunque seguramente me deje nombres en el tintero.

En primer lugar, quiero dar las gracias a Pierre quien comenzó siendo mi director de tesis y a quien a día de hoy considero un amigo. Pierre, gracias por darme la oportunidad de realizar mis primeras prácticas en IMDEA y por ayudarme a realizar mi primera estancia fuera de casa. Aquella experiencia me hizo descubrir que quería hacer un doctorado y fue tu interés y confianza en mi lo que me llevó a hacerlo en IMDEA. Gracias por guiarme con paciencia y apoyarme en mis decisiones durante estos 4 años, especialmente en mi interés por realizar estancias para conocer gente y lugares. Gracias a eso tuve el placer de trabajar con Rupak en Kaiserslatuern, con Javier en Munich y con Yann en San Francisco. Gracias también a ellos tres, y a los compañeros que tuve en esos viajes, en especial a Isa, Harry, Filip, Dmitry, Rayna, Marko, Bimba, Nick y Felix, por hacer de mis visitas grandes experiencias llenas de buenos recuerdos.

Quiero dar las gracias, también, a todo el personal del Instituto IMDEA Software. Ha sido un placer llevar a cabo mi trabajo rodeado de grandes profesionales en todos los ámbitos. Gracias Paloma, Álvaro, Felipe, Miguel, Isabel, Kyveli, Joaquín, Germán, Platón y Srdjan, entre otros, por ser los artífices de tantos buenos recuerdos. Especialmente, quiero agradecer a Ignacio su humor, su ayuda prestada durante estos últimos años y su paciencia al leer múltiples versiones de la introducción de este trabajo. Gracias por ser ese amigo del despacho 
de al lado al que ir a molestar siempre que quería comentar alguna idea, por tonta que fuera.

Elena, creo que ha sido una experiencia estupenda haber compartido mis años de universidad y de doctorado con una amiga como tú. He disfrutado muchísimo de todas las ocasiones en que hemos podido trabajar juntos y creo que hacíamos un equipo estupendo.

A mis profesores de bachillerato Soraya y Mario. Con vosotros entendí que estudiar era mucho más que aprobar un examen y me hicisteis disfrutar aprendiendo. Despertasteis en mi la pasión por aprender y por afrontar nuevos retos y fue esa pasión la que me llevó a estudiar el Doble Grado de Matemáticas con Informática y a realizar posteriormente un doctorado.

A mi familia, que recientemente creció en número, por el mero hecho de estar ahí. Gracias en especial a mi prima la Dra. Ana por ser la pionera, la primera investigadora y Dra. en la familia, que me ahorró el esfuerzo de explicar a todos cómo funciona el mundo de la investigación en que nos movemos.

A mis amigos de siempre y a los más recientes. Gracias por tantos buenos momentos, por visitarme cuando estaba fuera y por los viajes y planes que aún quedan por hacer. Creo firmemente que haber sido feliz en mi vida personal ha sido una pieza clave de mis éxitos profesionales. Quiero dar las gracias por ello a Alberto, Carlos, David, Rubén, Antonio, Victor, Eduardo, Álvaro, Guillermo, Cristina, Lara e Irene, entre muchos otros.

A mis padres, gracias por hacerme ser quien soy y por apoyarme siempre aún sin terminar de entender la aventura en la que me embarcaba al iniciar el doctorado. Gracias a vosotros he tenido una vida llena de facilidades, que me ha permitido centrarme siempre en mis estudios y mi trabajo. Cada uno de mis logros es resultado de vuestro esfuerzo.

Por último, quiero dar las gracias mi mujer. Jimena, gracias por apoyarme durante este tiempo, por acompañarme en mis viajes siempre que fue posible y por soportar la distancia cuando no. Gracias, en definitiva, por estar ahí. 


\section{Contents}

1 Introduction 1

1.1 The Contributions of This Dissertation . . . . . . . . . . 3

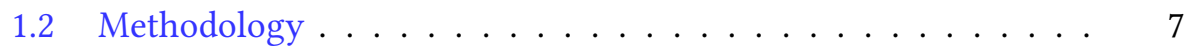

1.2.1 Quasiorders for Deciding Language Inclusion . . . . . 8

1.2.2 Quasiorders for Searching on Compressed Text . . . . 10

1.2.3 Quasiorders for Building Residual Automata . . . . . . . 10

2 State of the Art $\quad 13$

2.1 The Language Inclusion Problem . . . . . . . . . . . . . . . 13

2.1 .1 Antichains Algorithms . . . . . . . . . . . . 14

2.1.2 Solving Language Inclusion through Abstractions . . . . 16

2.2 Searching on Compressed Text . . . . . . . . . . . . 17

2.3 Building Residual Automata _. . . . . . . . . . . . . . . 19

3 Background $\quad 21$

3.1 Words and Languages . . . . . . . . . . . . . . . . . . . . . . . . 21

3.2 Finite-state Automata . . . . . . . . . . . . . . . . . 22

3.3 Context-free Grammars . . . . . . . . . . . . . . . . . . 26

3.4 Quasiorders . . . . . . . . . . . . . . . . 27

3.5 Kleene Iterates . . . . . . . . . . . . . . . . . . . 30

3.6 Closures and Galois Connections . . . . . . . . . . . . . 31

3.7 Complexity Notation . . . . . . . . . . . . . . . . 34 
4 Deciding Language Inclusion $\quad 35$

4.1 Inclusion Check by Complete Abstractions . . . . . . . . . . . 37

4.2 An Algorithmic Framework for Language Inclusion . . . . . . . 38

4.2.1 Languages as Fixed Points . . . . . . . . . . . . . 38

4.2.2 Abstract Inclusion Check using Closures . . . . . . . . . 40

4.2.2.1 Right Concatenation ........... 41

4.2.3 Solving the Abstract Inclusion Check . . . . . . . . . . 43

4.2.3.1 Using Finite Languages . . . . . . . . . . . . . 43

4.2.3.2 Using Galois Connections . . . . . . . . . . . 46

4.3 Instantiating the Framework . . . . . . . . . . . . . . 48

4.3.1 Word-based Abstractions . . . . . . . . . . 48

4.3.1.1 Right Concatenation .......... 51

4.3.2 Nerode Quasiorders ............... 51

4.3.2.1 On the Complexity of Nerode's quasiorders . 53

4.3.3 State-based Quasiorders ............. . 54

4.3.3.1 Inclusion in Regular Languages. . . . . . . . . 54

4.3.3.2 Simulation-based Quasiorders. . . . . . . 56

4.3.4 Inclusion in Traces of One-Counter Nets. . . . . . . . 58

4.4 A Novel Perspective on the Antichain Algorithm . . . . . . . . . 61

4.4.1 Relationship to the Antichains Algorithm . . . . . . . . 64

4.5 Inclusion for Context Free Languages . . . . . . . . . . . . . . 69

4.5.1 Extending the Framework to CFGs . . . . . . . . . . 70

4.5.2 Solving the Abstract Inclusion Check using Finite Languages . . . . . . . . . . . . . . 73

4.5.3 Solving the Abstract Inclusion Check using Galois Connections ................... 75

4.5.4 Instantiating the Framework . . . . . . . . . . 76

4.5.4.1 Myhill and State-based Quasiorders . . . . . 78

4.5.5 A Systematic Approach to the Antichain Algorithm . . . 82

4.6 An Equivalent Greatest Fixpoint Algorithm . . . . . . . . . . . . 86

5 Searching on Compressed Text $\quad 91$

5.1 Finding the Matches . . . . . . . . . . . . . . . . . 94

5.2 Counting Algorithm . . . . . . . . . . . . . 96

5.2.1 Data Structures . . . . . . . . . . . . . . . 99

5.3 Implementation . . . . . . . . . . . . . . . . 100

5.4 Empirical Evaluation . . . . . . . . . . . . . . 101

5.4.1 Tools .................... 101

5.4.2 Files and Regular Expressions . . . . . . . . . . . . 103

5.4.3 Analysis of the Results. . . . . . . . . . . . . 104 
5.5 Fine-Grained Analysis of the Implementation . . . . . . . . . . 106

5.5.1 Processing the Axiom Rule. . . . . . . . . . . . . . . 106

5.5.2 Number of Operations Performed by the Algorithm . . . 107

5.6 Fine-Grained Complexity . . . . . . . . . . . . . . . . . . 108

5.6.1 Complexity of Searching on Compressed Text . . . . . . 109

5.6.2 Complexity of Our Implementation . . . . . . . . . . . 111

6 Building Residual Automata $\quad 115$

6.1 Automata Constructions from Quasiorders . . . . . . . . . . 117

6.1.1 On the Size of $\mathrm{H}^{r}\left(\leqslant^{r}, L\right)$ and $\mathrm{H}^{\ell}\left(\leqslant^{\ell}, L\right) \ldots \ldots \ldots$

6.2 Language-based Quasiorders and their Approximation using NFAs 124

6.2.1 Automata Constructions . . . . . . . . . . . . . . 127

6.3 Double-Reversal Method for Building the Canonical RFA . . . . 133

6.3.1 Double-reversal Method . . . . . . . . . . . . . . 134

6.3.2 Generalization of the Double-reversal Method . . . . . . 135

6.3.2.1 Co-atoms and co-rests . . . . . . . . . 136

6.3.2.2 Tamm's Generalization of the Double-reversal Method for RFAs . . . . . . . . . . . 136

6.4 Learning Residual Automata . . . . . . . . . . . . . . . . . 137

6.4.1 The NL* Algorithm [Bollig et al. 2009] . . . . . . . . . . 138

6.4.2 The $\mathrm{NL}^{\leqslant}$Algorithm . . . . . . . . . . . . . . . . . 139

7 Future Work $\quad 145$

7.1 The Language Inclusion Problem . . . . . . . . . . . . . . 145

7.1.1 Language Inclusion Through Alternating Automata . . . 146

7.2 The Complexity of Searching on Compressed Text . . . . . . . . 149

7.3 The Performance of Residualization . . . . . . . . . . . . . 151

7.3.1 Reducing RFAs with Simulations . . . . . . . . . . . 151

8 Conclusions 153

$\begin{array}{ll}\text { Bibliography } & 159\end{array}$

$\begin{array}{ll}\text { Alphabetical Index } & 171\end{array}$ 



\section{List OF Figures}

1.1 Automata accepting the set of binary encodings of numbers divisible by 4 (top left), divisible by two (top right) and the product of these two automata (bottom). . . . . . . . . . . . .

1.2 Minimal DFA (left) and NFA (right) accepting the words in the alphabet $\{0,1\}$ of length 6 that contains two 1's separated by two symbols. For clarity, we use colors red, blue and black for transitions with labels " 0 ", " 1 " and " 0,1 ", respectively. . . . . . . . . .

1.3 Illustration of our quasiorder-based approach for deciding the language inclusion problems $L_{1} \subseteq L_{2}$ and $L_{3} \subseteq L_{2}$. . . . . . . .

1.4 The image on the left shows the principals induced by a quasiorder. Each arrow of the form $\rho(x) \stackrel{a}{\rightarrow} \rho(y)$ indicates that $\rho(x) a \subseteq \rho(y)$. For clarity, we show on the right the automaton resulting from the relation between the principals. . . . . . . 11

3.1 An NFA $\mathcal{N}$ with $\Sigma=\{a, b\}$ and $\mathcal{L}(\mathcal{N})=\Sigma^{*} a \Sigma a \Sigma^{*} \ldots \ldots 23$

3.2 DFA $\mathcal{N}^{D}$ obtained by determinizing the NFA from Figure 3.1. . $\quad 24$

3.3 RFA $\mathcal{N}^{\text {res }}$ obtained when residualizing to the NFA from Figure 3.1. 26

$4.1 \quad$ An NFA $\mathcal{N}$ with $\mathcal{L}(\mathcal{N})=\left(a+\left(b^{+} a\right)\right)^{*} \ldots \ldots 39$

4.2 Two automata $\mathcal{N}_{1}$ (left) and $\mathcal{N}_{2}$ (right) generating the regular languages $\mathcal{L}\left(\mathcal{N}_{1}\right)=a^{*}(a+b+c)$ and $\mathcal{L}\left(\mathcal{N}_{2}\right)=a^{*}\left(a(a+b)^{*} a+a^{+} c+\right.$ $a b+b b) \ldots \ldots \ldots \ldots 53$

4.3 A finite automaton $\mathcal{N}$ with $\mathcal{L}(\mathcal{N})=\left(b+a b^{*} a\right)(a+b)^{*}$. . . . . 79 
5.1 List of grammar rules (left) generating the string "ab\$a $\$ \mathrm{bab} \$ \mathrm{a}$ $\$ \mathrm{~b}$ ” (and no other) as evidenced by the parse tree (right). . . . .

5.2 NFAs $\mathcal{N}^{\prime}$ (left) and $\mathcal{N}$ (right) on $\Sigma=\{a, b, \$\}$ with $\mathcal{L}\left(\mathcal{N}^{\prime}\right)=$ $\{a b, b b\}$ and $\mathcal{L}(\mathcal{N})=\Sigma^{*} \cdot \mathcal{L}\left(\mathcal{N}^{\prime}\right) \cdot \Sigma^{*} \ldots \ldots \ldots \ldots$

5.3 Data structures enabling nearly optimal running time for Algorithm CountLines. The image shows the contents of $\mathcal{M}$ after processing rule $X_{i} \rightarrow \alpha_{i} \beta_{i}$ and the contents of $\mathcal{K}$ after processing $X_{\ell} \rightarrow \alpha_{\ell} \beta_{\ell}$ with $\beta_{\ell}=X_{i} \ldots \ldots \ldots \ldots \ldots$

5.4 The first graph shows the time required to report the number of lines in a log file matching a regular expression. All tools are fed with the same regular expression. The decompress and search approach is implemented in parallel i.e. searching on the output uncompressed text as it is recovered by the decompressor. As a reference, we show the time required for decompressing the file with different tools (horizontal lines). The second graph is the cactus plot corresponding the the data from the first graph. In this case, we observe that zearch is faster than any other tool, except grep. . . . . . . . . . . . . . . . .

5.5 Average running time required to report the number of lines matching a regular expression in a file and time required for decompression. Colors indicate whether the tool performs the search on the uncompressed text (blue); the compressed text (black); the output of the decompressor (green); or decompresses the file without searching (red). . . . . . . . . . . . . . . 104

5.6 DFA for the regular expression " $a+b+b+a+c+"=" a+b b+a+c+" . \quad . \quad 112$

5.7 DFA for the regular expression " $\mathrm{a} * \mathrm{~b} * \mathrm{~b} * \mathrm{a} * \mathrm{c} *$ " $=$ " $\mathrm{a} * \mathrm{~b} * \mathrm{a} * \mathrm{c} *$ ". . . 113

5.8 DFA for the regular expression " $(\mathrm{a} \mid \mathrm{b})(\mathrm{a} \mid \mathrm{c})(\mathrm{b} \mid \mathrm{c})(\mathrm{a} \mid \mathrm{c})$ ”. . . 113

6.1 Relations between the constructions $\operatorname{Res}^{\ell}, \operatorname{Res}^{r}, \mathrm{Can}^{\ell}$ and $\mathrm{Can}^{r}$. Note that constructions $\mathrm{Can}^{r}$ and $\mathrm{Can}^{\ell}$ are applied to the language generated by the automaton in the origin of the labeled arrow while constructions $\operatorname{Res}^{r}$ and $\operatorname{Res}^{\ell}$ are applied directly to the automaton. . . . . . . . . . . . . . .

6.2 Form left to right: an NFA $\mathcal{N}$ and the RFAs $\mathcal{N}^{\text {res }}$ and $\operatorname{Res}^{r}(\mathcal{N})$. We omit the empty states for clarity. . . . . . . . . . . .

7.1 Alternating automaton $\mathcal{A}$ generating the language $\mathcal{L}(\mathcal{A})=a(a+$ $b)^{*} \ldots \ldots \ldots \ldots \ldots \ldots \ldots \ldots$ 
7.2 From left to right, grammars built by the compression algorithms sequitur [Nevill-Manning and Witten 1997], repair [Larsson and Moffat 1999] and LZW [Welch 1984] for "xabcdbcabcdbcy". . 150

8.1 Summary of the existing results about the generalized doublereversal method for building the minimal DFA (first row) and the canonical RFA (second row) for a given language. The results on the first column are based on the notion of atoms of a language while the results on the second column are based on quasiorders. 



\section{LIST OF TABLES}

4.1 Summary of the quasiorders that should be used within our framework, i.e. using Theorem 4.2.11, to derive the different antichains algorithms that are (explicitly or implicitly) given by Wulf et al. [2006]. Each cell of the form $f(u) \subseteq f(v)$ is the definition of the quasiorder $u \leqslant v \stackrel{\text { def }}{=} f(u) \subseteq f(v)$ that should be used to derive the antichains algorithm given by the column for solving the language inclusion given by the row. . . . . . . . . .

5.1 Sizes (in $\mathrm{MB}$ ) of the compressed files and (de)compression times (in seconds). Maximum compression levels enabled. (Blue = best; bold black $=$ second best; red $=$ worst $). \ldots \ldots \ldots \ldots$

5.2 Sizes (in KB) of the compressed files and (de)compression times (in seconds). Maximum compression levels enabled. (Blue = best; bold black $=$ second best; red $=$ worst). . . . . . . . . . . 105

5.3 Time (ms) required to report the number of lines matching a regular expression in the $500 \mathrm{MB}$ large contrived file. (Blue = fastest; bold black $=$ second fastest; red $=$ slowest $). \ldots \ldots \ldots$. . . .

5.4 Analysis of the values $\tilde{s}_{\ell}$ and $s_{(\sigma)_{i}}$ obtained when considering different regular expressions to search Subtitles (100 MB uncompressed long). The fifth column of the fourth row indicates that when considering the expression " $I$ * you", for $75 \%$ of the grammar rules we have $\tilde{s}_{\ell} \leq 13$ while $s^{3}=729 \ldots \ldots \ldots$. . . 107 



\section{List of Publications}

This thesis comprises the following four papers for which I am the main author. The first two have been published in top peer-reviewed academic conferences while the last two have recently been submitted and have not been published yet:

1. Pedro Valero and Dr. Pierre Ganty

Regular Expression Search on Compressed Text

Published in Data Compression Conference, March 2019.

2. Pedro Valero, Dr. Pierre Ganty and Prof. Francesco Ranzato

Language Inclusion Algorithms as Complete Abstract Interpretations

Published in Static Analysis Symposium, October 2019.

3. Pedro Valero, Dr. Pierre Ganty and Elena Gutiérrez

A Quasiorder-based Perspective on Residual Automata

Submitted to the International Symposium on Mathematical Foundations of Computer Science, May 2020.

4. Pedro Valero, Dr. Pierre Ganty and Prof. Francesco Ranzato Complete Abstractcions for Checking Language Inclusion Submitted to Transactions on Computational Logic, May 2020.

Using the techniques presented in first of the above mentioned papers, I developed a tool for searching with regular expressions in compressed text. The implementation is available on-line at https://github.com/pevalme/zearch. 
I have also contributed to the following papers which are not part of this thesis.

1. Elena Gutiérrez, Pedro Valero and Dr. Pierre Ganty

A Congruence-based Perspective on Automata Minimization Algorithms

Published in International Symposium on Mathematical Foundations of Computer Science, August 2019

2. Pedro Valero, Dr. Pierre Ganty and Boris Köpf

A Language-theoretic View on Network Protocols

Published in Automated Technology for Verification and Analysis, October 2017 


\section{INTRODUCTION}

Formal languages, i.e. languages for which we have a finite formal description, are used to model possibly infinite sets so that their finite descriptions can be used to reason about these sets. As a consequence, Formal Language Theory, i.e. the study of formal languages and the techniques for manipulating their finite representations, finds applications in several domains in computer science.

For example, the possibly infinite set of assignments that satisfy a given formula in some logic can be seen as a formal language whose finite description is the formula itself. In some logics, the set of values that satisfy any formula is regular and, therefore, it can be described by means of a finite-state automaton (automaton for short). When this is the case, it is possible to reason in that logic by manipulating automata as shown in Example 1.1.

Example 1.1. Consider the formulas $f_{2}:$ " $x \bmod 2=0 "$ and $f_{4}:$ : $x \bmod 4=0$ ". Next we show how to reason about the formula $f_{42}:$ : $f_{4} \wedge f_{2}$ " by means of automata.

A binary sequence " $x$ " encodes a number divisible by 4 iff the last two digits are 0 's. Similarly, " $x$ " encodes a number divisible by 2 iff the last digit is 0 . Therefore, the automata $A_{4}$ and $A_{2}$ from Figure 1.1 accept the binary encodings of numbers " $x$ " that satisfy the formulas $f_{4}$ : " $x \bmod 4=0$ " and $f_{2}:$ : $x \bmod 2=0$ ", respectively.

Since the numbers satisfying the formula $f_{42}$ are, by definition, the ones satisfying both $f_{4}$ and $f_{2}$, the automaton for $f_{42}$ is $A_{42}=A_{2} \times A_{4}$, shown in Figure 1.1, which recognizes exactly the encodings accepted by both $A_{2}$ and $A_{4}$. Therefore, there exists a number satisfying $f_{42}$ iff the language accepted by $A_{42}$ is not empty.

On the other hand, since the automaton $A_{4}$ accepts a language that is included in the one of $A_{2}$, we conclude that the encodings satisfying $f_{4}$ also satisfy $f_{2}$. Therefore, the automaton for $A_{4}$ is equivalent to, i.e. it accepts the same language as, the automaton $A_{42}$ and both are automata for $f_{42}$. 


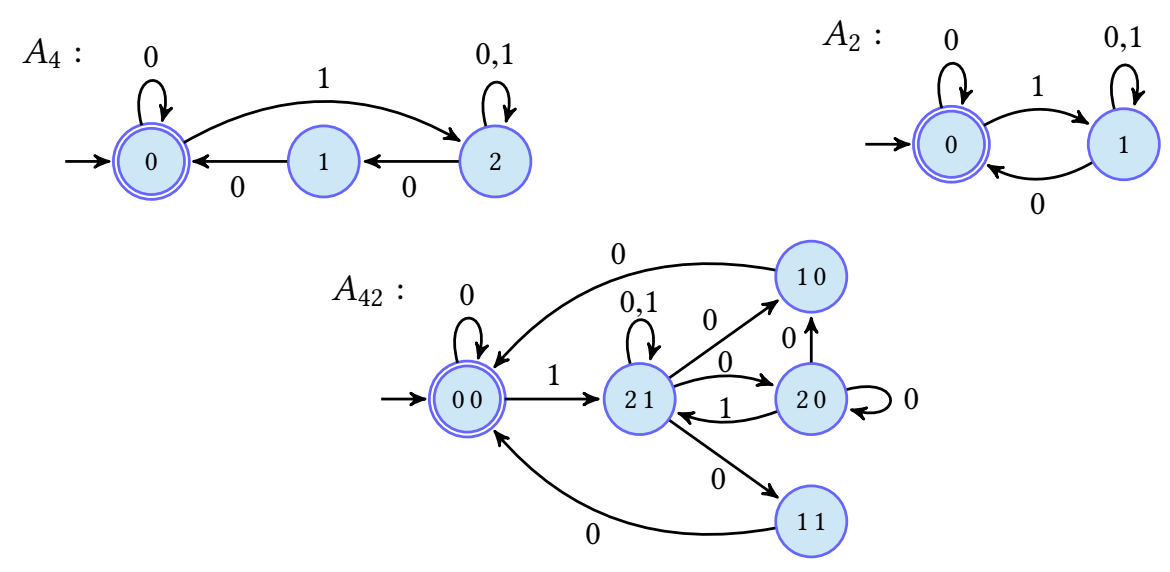

Figure 1.1: Automata accepting the set of binary encodings of numbers divisible by 4 (top left), divisible by two (top right) and the product of these two automata (bottom).

This idea led to the development of automata-based decision procedures for logical theories such as Presburger arithmetic [Wolper and Boigelot 1995] and the Weak Second-order theory of One or Two Successors (WS1S/WS2S) [Henriksen et al. 1995; Klarlund 1999] among others [Allouche et al. 2003; Schaeffer 2013].

A similar idea is used in regular model checking [To and Libkin 2008; Abdulla 2012; Clarke et al. 2018], where formal languages are used to describe the possibly infinite sets of states that a system might reach during its execution.

A different use of formal languages in computer science is the lossless compression of textual data [Charikar et al. 2005; Hucke et al. 2016]. In this scenario the data is seen as a language consisting of a single word and its finite formal description as a grammar is seen as a succinct representation of the language it generates. As the following example evidences, the grammar might be exponentially smaller than the data.

Example 1.2. Let $k$ be an integer greater than 1 and let $\mathcal{G}$ be the grammar with the set of variables $\left\{X_{i} \mid 0 \leq i \leq k\right\}$, alphabet $\{a\}$, axiom $X_{k}$ and the set of rules $\left\{X_{i} \rightarrow X_{i-1} X_{i-1} \mid 1 \leq i \leq k\right\} \cup\left\{X_{0} \rightarrow a\right\}$.

Clearly, $\mathcal{G}$ has size linear in $k$ and produces the word $a^{2^{k}}$. Therefore, the grammar is exponentially smaller than the word it generates.

The idea of using grammars to compress textual data has led to the development of several grammar-based compression algorithms [Ziv and Lempel 1978; Nevill-Manning and Witten 1997; Larsson and Moffat 1999]. These algorithms offer some advantages with respect to other classes of compression techniques, such as the ones based on the well-known LZ77 algorithm [Ziv and Lempel 1977], 
in terms of the structure of the compressed representation of the data (which is a grammar). In particular, they allow us to analyze the uncompressed text, i.e. the language, by looking at the compressed data, i.e. the grammar [Lohrey 2012].

\subsection{The Contributions of This Dissertation}

In this dissertation we focus on three problems from Formal Language Theory: deciding language inclusion, searching on grammar-compressed text and building residual automata. As we describe next, these are well-studied and important problems in computer science for which there are still challenges to overcome.

\section{The Language Inclusion Problem}

In the first two scenarios described before, i.e. automata-based decision procedures and regular model checking, the language inclusion problem, i.e. deciding whether the language inclusion $L_{1} \subseteq L_{2}$ holds, is a fundamental operation.

For instance, in Example 1.1, deciding the language inclusion between the languages generated by automata $A_{4}$ and $A_{2}$ allows us to infer that all values satisfying $f_{4}$ also satisfy $f_{2}$. Similarly, in the context of regular model checking, we can define a possibly infinite set of "good" states that the system should never leave and solve a language inclusion problem to decide whether the system is confined to the set of good states.

As a consequence, the language inclusion problem is a fundamental and classical problem in computer science [Hopcroft and Ullman 1979, Chapter 11]. In particular, language inclusion problems of the form $L_{1} \subseteq L_{2}$, where both $L_{1}$ and $L_{2}$ are regular languages, appear naturally in different scenarios as the ones previously described.

The standard approach for solving such problems consists on reducing them to emptiness problems using the fact that $L_{1} \subseteq L_{2} \Leftrightarrow L_{1} \cap L_{2}^{c}=\varnothing$. However, algorithms implementing this approach suffer from a worst case exponential blowup when computing $L_{2}^{c}$ since it requires determinizing the automaton for $L_{2}$. The state of the art approach to overcome this limitation is to keep the computation of the automaton for $L_{2}^{c}$ implicit, thus preventing the exponential blowup for many instances of the problem.

For instance, Wulf et al. [2006] developed an algorithm for deciding language inclusion between regular languages that uses antichains, i.e. sets of incomparable elements, to reduce the blowup resulting from building the complement of a given automaton. Their work was later improved by Abdulla et al. [2010] and Bonchi and Pous [2013] who used simulations between the states of the automata to further reduce the blowup associated to the complementation step. Then, 
Holík and Meyer [2015] adapted the use of antichains to decide the inclusion of context-free languages into regular ones.

However, even though these algorithms have a common foundation, i.e. they all reduce the language inclusion problem to an emptiness one through complementation and use antichains to keep the complementation implicit, the relation between them is not well understood. This is evidenced by the fact that the generalization by Holík and Meyer [2015] of the antichains algorithm of Wulf et al. [2006] was obtained by rephrasing the inclusion problem as a data flow analysis problem over a relational domain.

Our Contribution. We use quasiorders, i.e. reflexive and transitive relations, to define a framework from which we systematically derive algorithms for deciding language inclusion such as the ones of Wulf et al. [2006] and Holík and Meyer [2015]. Indeed, we show that these two algorithms are conceptually equivalent and correspond to two instantiations of our framework using different quasiorders. Moreover, by using a quasiorder based on simulations between the states of an automata, we derive an improved antichains algorithm that partially matches the one of Abdulla et al. [2010].

Furthermore, our framework goes beyond inclusion into regular languages and allows us to derive an algorithm for deciding the language inclusion $L_{1} \subseteq L_{2}$ when $L_{1}$ is regular and $L_{2}$ is the set of traces of a one counter net, i.e. an automaton equipped with a counter that cannot test for 0 . Finally, we also derive a novel algorithm for deciding inclusion between regular languages.

\section{Searching on Compressed Text}

The growing amount of information handled by modern systems demands for efficient techniques both for compression, to reduce the storage cost, and for regular expression searching, to speed up querying.

Therefore, the problem of searching on compressed text is of practical interest as evidenced by the fact that state of the art tools for searching with regular expressions, such as grep ${ }^{1}$ and ripgrep $^{2}$, provide a method for searching on compressed files by decompressing them on-the-fly.

Due to the high performance of state of the art compressors such as zstd and $1 z 4^{4}$, the performance of searching on the decompressed data as it is recovered by the decompressor is comparable with that of searching on the un-

\footnotetext{
${ }^{1}$ https://www.gnu.org/software/grep/manual/grep.html.

${ }^{2}$ https://github.com/BurntSushi/ripgrep.

${ }^{3}$ https://github.com/facebook/zstd

${ }^{4}$ https://github.com/lz4/lz4
} 
compressed data. Therefore, the parallel decompress-and-search approach is the state of the art for searching on compressed text.

However, when using a grammar-based compression technique it is possible to manipulate the compressed data, i.e. the grammar, to analyze the uncompress data, i.e. the language generated by the grammar. Intuitively, this means that the information about repetitions in the text present in its compressed version can be used to enhance the search. Therefore, searching on grammar-compressed text could be even faster than searching on the uncompressed text.

This idea is exploited by multiple algorithms that perform certain operations directly on grammar-compressed text, i.e. without having to recover the uncompressed data, such as finding given words [Navarro and Tarhio 2005], finding words that match a given regular expression [Navarro 2003; Bille et al. 2009] or finding approximate matches [Navarro 2001].

Nevertheless, the implementations of Navarro [2003] and Navarro and Tarhio [2005] (to the best of our knowledge, the only existing tools for searching on compressed text) are not faster than the state of the art decompress and search approach. Partly, this due to the fact that these algorithms only apply to data compressed with one specific grammar-based compressor, namely LZ78 [Ziv and Lempel 1978], which, as shown by Hucke et al. [2016], cannot achieve exponential compression ratios ${ }^{5}$.

Our Contribution. We improve this situation by rephrasing the problem of searching on compressed text as a language inclusion problem between a contextfree language (the text) and a regular one (the expression). Then, we instantiate our quasiorder-based framework for solving language inclusion and adapt it to the specifics of this scenario, where the context-free grammar generates a single word: the uncompressed text. The resulting algorithm is not restricted to any class of grammar-based compressors and it reports the number of lines in the text containing a match for a given expression in time linear with respect to the size of the compressed data.

We implement this algorithm in a tool -zearch ${ }^{6}-$ for searching with regular expressions in grammar-compressed text. The experiments evidence that compression can be used to enhance the search and, therefore, the performance of zearch improves with the compression ratio of the data. Indeed, our tool is as fast as searching on the uncompressed data when the data is well-compressible, i.e. it results in compression ratio above 13 , which occurs, for instance, when considering automatically generated $\log$ files.

\footnotetext{
${ }^{5}$ The compression ratio for a file of size $T$ compressed into size $t$ is $T / t$.

${ }^{6}$ https://github.com/pevalme/zearch
} 


\section{Building Residual Automata}

Clearly, the problem of finding a concise representation of a regular language is also a fundamental problem in computer science.

There exists two main classes of automata representations for regular languages, both having the same expressive power: non-deterministic (NFA for short) and deterministic (DFA for short) automata. While DFAs are simpler to manipulate than NFAs ${ }^{7}$ they are, in the worst case, exponentially larger.

Example 1.3. The minimal DFA for the set of words of length $2 n+2$ with two 1's separated by $n$ symbols has size exponential in $n$ since any DFA for that language must have one state for each of the $2^{n}$ possible prefixes of length $n$. Figure 1.2 shows the minimal DFA and an exponentially smaller NFA for the case $n=2$.
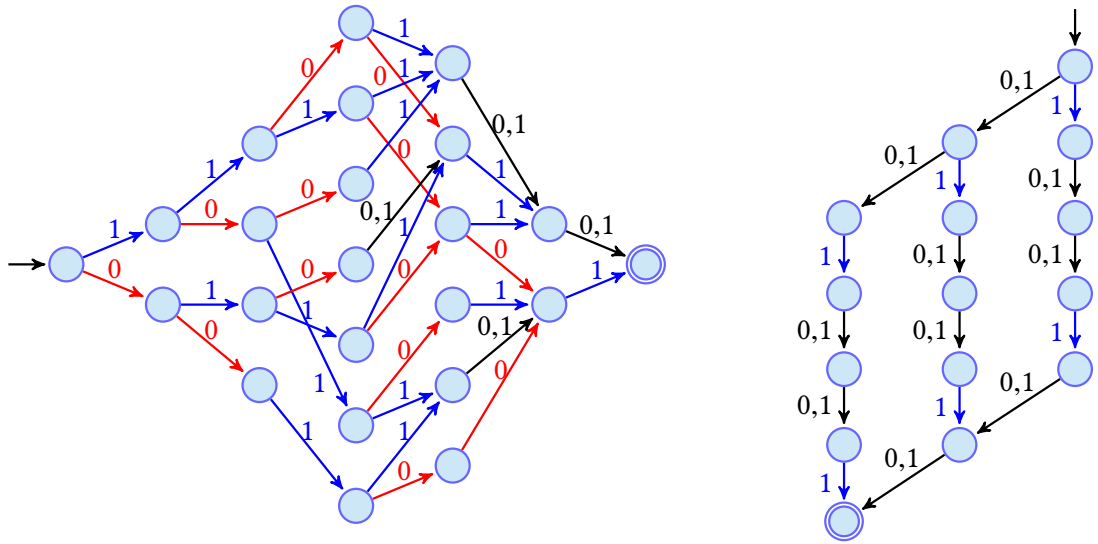

Figure 1.2: Minimal DFA (left) and NFA (right) accepting the words in the alphabet $\{0,1\}$ of length 6 that contains two 1's separated by two symbols. For clarity, we use colors red, blue and black for transitions with labels "0", "1" and "0,1", respectively.

Therefore, algorithms relying on determinized automata, such as the standard algorithm for building the complement of an NFA, do not scale despite the existence of different techniques for reducing the size of DFAs [Hopcroft 1971; Moore 1956] and for building DFAs of minimal size [Sakarovitch 2009; Adámek et al. 2012; Brzozowski and Tamm 2014].

This has led to the introduction of residual automata [Denis et al. 2001; 2002] (RFA for short) as a generalization of DFAs that breaks determinism in favor of conciseness of the representation. Therefore, RFAs are easier to manipulate

\footnotetext{
${ }^{7}$ For instance, in order to build the complement of a DFA it suffices to switch final and non-final states while complementing an NFA requires determinizing it.
} 
than NFAs (there exists a canonical minimal RFA for every regular language, which makes learning easier) and more concise than DFAs (both automata from Figure 1.2 are RFAs). These properties make RFAs specially appealing in certain domains such us Grammatical Inference [Denis et al. 2004; Bollig et al. 2009].

There exists a clear relationship between RFAs and DFAs as evidenced by the similarities between the residualization and determinization operations and the fact that a straightforward modification of the double-reversal method for building minimal DFAs yields a method for building minimal RFAs. However, the connection between these two formalisms is not fully understood as evidenced by the fact that the relation between the generalization of the double-reversal methods for DFAs [Brzozowski and Tamm 2014] and RFAs [Tamm 2015] is not immediate.

Our Contribution. We present a framework of quasiorder-based automata constructions that yield residual and co-residual automata. We find that one of these constructions defines a residualization operation that produces smaller automata than the one of Denis et al. [2002] and for which the double-reversal method holds: residualizing a co-residual automaton yields the canonical RFA. Moreover, we derive a generalization of this double-reversal method for RFAs, along the lines of the one of Brzozowski and Tamm [2014] for DFAs that is more general than the one of Tamm [2015].

Incidentally, we also evidence the connection between the generalized doublereversal method for RFAs of Tamm [2015] and the one of Brzozowski and Tamm [2014] for DFAs. Finally, we offer a new perspective of the NL* algorithm of Bollig et al. [2009] for learning RFAs as an algorithm that iteratively refines a quasiorder and uses our automata constructions to build RFAs.

\subsection{Methodology}

The contributions of this thesis, described in the previous section, are the result of using monotone well-quasiorders, i.e. quasiorders that satisfy certain properties with respect to concatenation of words and for which there is no infinite decreasing sequence of elements, as building blocks for tackling problems from Formal Language Theory.

Monotone well-quasiorders have proven useful for reasoning about formal languages from a theoretical perspective (see the survey of D'Alessandro and Varricchio [2008]). For instance, Ehrenfeucht et al. [1983] showed that a language is regular iff it is closed for a monotone well-quasiorder and de Luca and Varricchio [1994] extended this result by showing that a language is regular iff it is closed for a left monotone and for a right monotone well-quasiorders. On the 
other hand, Kunc [2005] used well-quasiorders to show that all maximal solutions of certain systems of inequalities on languages are regular.

Our work evidences that monotone well-quasiorders also have practical applications by placing them at the core of some well-known algorithms.

\section{Monotone Well-Quasiorders}

Quasiorders are binary relations that are reflexive, i.e. every word is related to itself, and transitive, i.e. if a word " $\mathrm{u}$ " is related to " $\mathrm{v}$ " which is related to "w" then " $\mathrm{u}$ " is related to " $\mathrm{w}$ ".

Intuitively, we use quasiorders to group words that behave "similarly" (in a certain way) with respect to a given regular language. This naturally leads to the use of monotone quasiorders so that "similarity" between words is preserved by concatenation, i.e. when concatenating two "similar" words with the same letter the resulting words remain "similar".

Example 1.4. Consider the length quasiorder, which says that " $u$ " is related to " $v$ " iff $|u| \leq|v|$ where $|u|$ denotes the length of a word " $u$ ".

It is straightforward to check that this is a monotone quasiorder since

(i) $|u| \leq|u|$ for every word $u$, hence it is reflexive;

(ii) if $|u| \leq|v|$ and $|v| \leq|w|$ then $|u| \leq|w|$, hence it is transitive;

(iii) if $|u| \leq|v|$ then $|u a| \leq|v a|$ for every letter a, hence it is monotone.

The most basic sets of words that can be formed by using a quasiorder are the so called principals, i.e. sets of words that are related to a single one which we refer to as the generating word of the principal. For example, given the length quasiorder, the principal with generating word "u" is the set of all words "w" with $|\mathrm{u}| \leq|\mathrm{w}|$.

Finally, when considering well-quasiorders we find that the union of the principals of any (possibly infinite) set of words coincides with the union of the principals of a finite subset of words. For instance, the quasiorder from Example 1.4 is a monotone well-quasiorder since the union of the principals of any infinite set of words coincides with the principal of the shortest word in the set.

Next, we offer a high-level description on how we use monotone well-quasiorders and their induced principals in each of the contributions of this thesis.

\subsubsection{Quasiorders for Deciding Language Inclusion}

Consider the language inclusion problem $L_{1} \subseteq L_{2}$ where $L_{1}$ is context-free and $L_{2}$ is regular. The principals of a given monotone well-quasiorder can be used to compute an over-approximation of $L_{1}$ that consists of a finite number of elements. 
If the quasiorder is such that a principal is included in $L_{2}$ iff its generating word is in $L_{2}$, then we can reduce the language inclusion problem $L_{1} \subseteq L_{2}$ to the simpler problem of deciding a finite number of membership queries for $L_{2}$. To do that it suffices to compute the over-approximation of $L_{1}$ and check membership in $L_{2}$ for the generating words of the finitely many principals that form the overapproximation. This approach is illustrated in Figure 1.3.

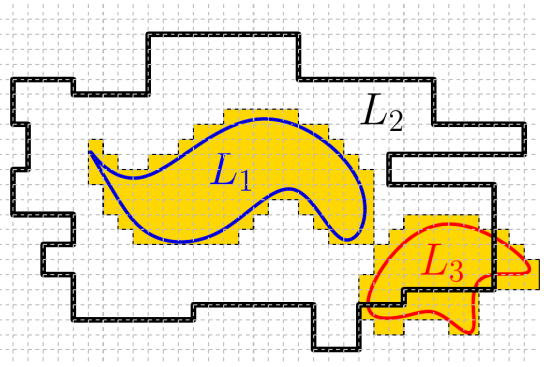

Given a monotone well-quasiorder whose principals are the dashed squares shown on the image on the left, we compute overapproximations (colored areas) of the languages $L_{1}$ and $L_{3}$. Since $L_{2}$ is a union of principals, the over-approximation of a language is included in $L_{2}$ iff the language is included in $L_{2}$. Therefore, we find that $L_{1} \subseteq L_{2}$ but $L_{3} \nsubseteq L_{2}$.

Figure 1.3: Illustration of our quasiorder-based approach for deciding the language inclusion problems $L_{1} \subseteq L_{2}$ and $L_{3} \subseteq L_{2}$.

In order to compute the over-approximation of $L_{1}$ we successively overapproximate the Kleene iterates of its least fixpoint characterization. The following example shows the language equations for a context-free language and the first steps of the Kleene iteration, which converges to the least fixpoint of the equations.

Example 1.5. Consider the language equations $\{X=a X \cup Y a \cup b Y, Y=a\}$, whose Kleene iterates converge to their least fixpoint:

$$
\left\{\begin{array} { l } 
{ X = \varnothing } \\
{ Y = \varnothing }
\end{array} \Rightarrow \left\{\begin{array} { l } 
{ X = \varnothing } \\
{ Y = \{ a \} }
\end{array} \Rightarrow \left\{\begin{array} { l } 
{ X = \{ a a , b a \} } \\
{ Y = \{ a \} }
\end{array} \Rightarrow \ldots \Rightarrow \left\{\begin{array}{l}
X=a^{*}(a a \mid b a) \\
Y=\{a\}
\end{array}\right.\right.\right.\right.
$$

This approach for solving language inclusion problems is studied in Chapter 4 . In that chapter we present a quasiorder-based framework which, by instantiating it with different monotone well-quasiorders, allows us to systematically derive well-known decision procedures for different language inclusion problems such as the antichains algorithms of Wulf et al. [2006] and Holík and Meyer [2015].

Moreover, by switching from least fixpoint equations for computing the overapproximation of $L_{1}$ to greatest fixpoint equations, we are able to obtain a novel algorithm for deciding language inclusion between regular languages. 


\subsubsection{Quasiorders for Searching on Compressed Text}

Searching with a regular expression in a grammar-compressed text ${ }^{8}$ amounts to deciding whether the language generated by a grammar, which consists of a single word, is included in a regular language. Therefore, we can apply the quasiorder-based framework described in the previous section, i.e. we can compute an over-approximation of the language generated by the grammar and check inclusion of the over-approximation into the regular language.

However this approach would only indicate whether there is a subsequence in the text that matches the expression and it would not produce enough information to count the matches let alone recover them.

In order to report the exact lines ${ }^{9}$ that contain a match (either count them or recover the actual lines), we need to compute some extra information for each variable of the grammar, beyond the over-approximation of the generated language. Indeed, we need to compute the following information regarding the language generated by each variable, which consists of a single word $^{10}, w$ :

(i) The number of lines that contain a match.

(ii) Whether there is a "new line" symbol in $w$.

(iii) Whether the prefix of $w$ contains a match.

(iv) Whether the suffix of $w$ contains a match.

This quasiorder-based approach is presented in Chapter 5 where we show that the above mentioned extra information for each variable of the grammar is trivially computed for the terminals and then propagated through all the variables until the axiom. Furthermore, Chapter 5 includes a detailed description of the implementation and evaluation of the resulting algorithm which, as the experiments show, outperforms the state of the art.

\subsubsection{Quasiorders for Building Residual Automata}

It is well-known that the construction of the minimal DFA for a language is related to the use of congruences, i.e. symmetric monotone quasiorders [Büchi 1989; Khoussainov and Nerode 2001].

\footnotetext{
${ }^{8}$ By "searching" we mean finding subsequences of the uncompressed text that match a regular expression, i.e. that are included in a given regular language.

${ }^{9} \mathrm{We}$ use the standard definition of line as a sequence of characters delimited by "new line" symbols.

${ }^{10}$ Recall that, in the context of grammar-based compression, the grammar is a compressed representation of a text, hence it generates a single word: the text. As a consequence, each variable of the grammar generates a single word.
} 
Recently, Ganty et al. [2019] generalized this idea and offered a congruencebased perspective on minimization algorithms for DFAs. Intuitively, they build automata by using the principals induced by congruences as states and define the transitions according to inclusions between the principals and the sets obtained by concatenating them with letters. When the congruence has finite index then it induces a finite number of principals and, therefore, the resulting automata have finitely many states. Figure 1.4 illustrates this automata construction.
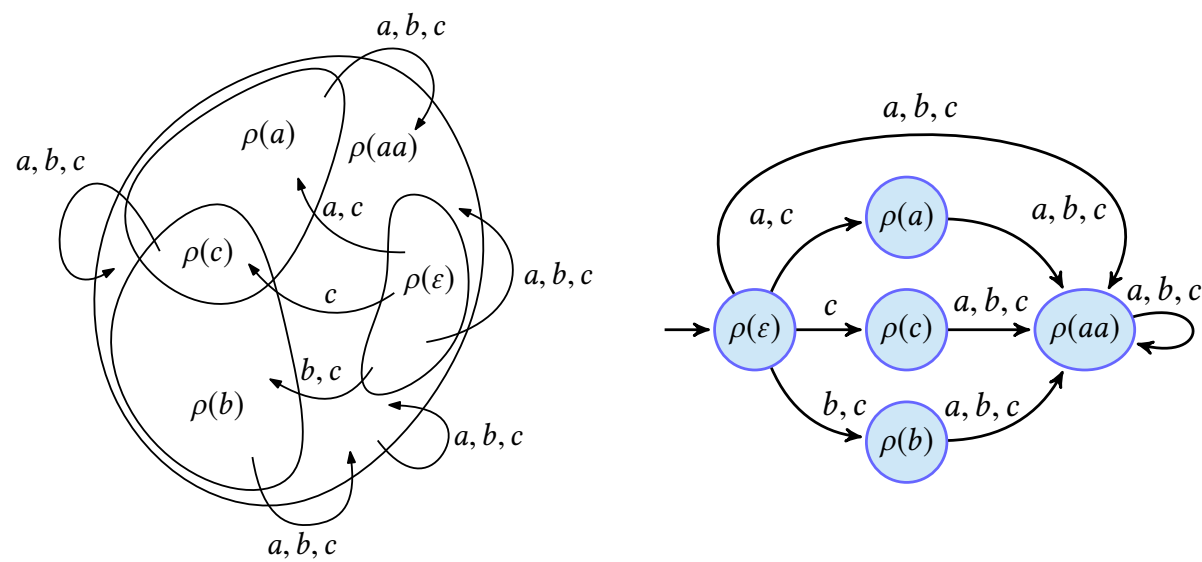

Figure 1.4: The image on the left shows the principals induced by a quasiorder. Each arrow of the form $\rho(x) \stackrel{a}{\rightarrow} \rho(y)$ indicates that $\rho(x) a \subseteq \rho(y)$. For clarity, we show on the right the automaton resulting from the relation between the principals.

Let $\rho(u)$ denote the principal for a word $u$. The monotonicity of congruences ensures that every set $\rho(u) a$ is included in a principal $\rho(v)$ and, since congruences are symmetric, the principals induced by a congruence are disjoint and, therefore, the resulting automata is deterministic. By switching from congruences to quasiorders we obtain possibly overlapping principals which enables non-determinism and allows us to obtain residual automata which, recall, are a generalization of DFAs. Clearly, the principals shown in Figure 1.4 correspond to a quasiorder rather than a congruence since they are not disjoint.

This quasiorder-based perspective on RFAs is presented in Chapter 6 where we define quasiorder-based automata constructions that yield RFAs or co-RFAs, depending on the properties of the input quasiorder. Moreover, given two comparable quasiorders, our automata construction instantiated with the coarser quasiorder yields a smaller automaton. This is to be expected since a coarser quasiorder induces fewer principals which, recall, are the states of the automata.

As a consequence, building the canonical minimal RFA for a given language amounts to instantiating our automata construction with the coarsest quasi- 
order that satisfies certain requirements. Interestingly, building the minimal DFA amounts to instantiating the framework of Ganty et al. [2019] with the coarsest congruence that satisfies the same requirements. As we shall see in Chapter 6, the congruence and the quasiorder used for building the minimal DFA and RFA, respectively, are closely related.

We conclude that monotone quasiorders are fundamental for RFAs as congruences are fundamental for DFAs, which evidences the relationship between these two classes of automata. 


\section{State of The Art}

In this dissertation, we present two quasiorder-based frameworks that allow us to systematically derive algorithms for solving different language inclusion problems and manipulating residual automata, respectively. Moreover, we show that our algorithms for deciding language inclusion can be adapted for searching on compressed text.

Our theoretical framework allows us to devise some novel algorithms and offer new insights on existing ones. Therefore, most of the works related to ours are briefly discussed within the following chapters, when explaining them within our quasiorder-based perspective. This is the case, specially, in Chapters 4 and 6 .

However, we present in this chapter a detailed description of some previous works in order to provide an overview of the state of the art for these problems before writing this Ph.D. Thesis.

\subsection{The Language Inclusion Problem}

Consider the language inclusion problem $L_{1} \subseteq L_{2}$. When the underlying representations of $L_{1}$ and $L_{2}$ are regular expressions, one can check language inclusion using some rewriting techniques [Antimirov 1995; Keil and Thiemann 2014], thus avoiding the translation of the regular expression into an equivalent automaton.

On the other hand, when the languages are given through finite automata, a well known and standard method to solve the language inclusion problem is to reduce it to a disjointness problem via the construction of the language complement: $L_{1} \subseteq L_{2}$ iff $L_{1} \cap L_{2}^{c}=\varnothing$. The bottleneck of this approach is the language complementation since it involves a determinization step which entails a worst case exponential blowup. 
In order to alleviate this bottleneck, Wulf et al. [2006] put forward a breakthrough result where complementation was sidestepped by a lazy construction of the determinized NFA, which provided a huge performance gain in practice. Their algorithm, deemed the antichains algorithm, was subsequently enhanced with simulation relations by Abdulla et al. [2010]. The current state of the art for solving the language inclusion problem between regular languages is the bisimulation up-to approach proposed by Bonchi and Pous [2013], of which the antichains algorithm and their enhancement with simulations can be viewed as particular cases.

\subsubsection{Antichains Algorithms}

The antichains algorithm of Wulf et al. [2006] was originally designed as an algorithm for solving the universality problem for regular languages, i.e. deciding whether $\Sigma^{*} \subseteq L$ holds when $L$ is regular.

Before the introduction of this algorithm, the standard approach for deciding universality of a regular language given its automaton was to determinize the automaton and check whether all states are final. The antichains algorithm improved this situation by keeping the determinization step implicit.

In their work, Wulf et al. [2006] also adapted their antichains algorithm for solving the language inclusion problem $L_{1} \subseteq L_{2}$ when both $L_{1}$ and $L_{2}$ are regular. Next, we describe this antichains algorithm for solving language inclusion.

Consider the inclusion problem $L_{1} \subseteq L_{2}$ and let $\mathcal{N}_{1}$ and $\mathcal{N}_{2}$ be finite-state automata generating the languages $L_{1}$ and $L_{2}$ respectively. The intuition behind the antichains algorithm is to compute, for each state $q$ of $\mathcal{N}_{1}$, the set $S_{q}$ of sets of states of $\mathcal{N}_{2}$ from which no final state of $\mathcal{N}_{2}$ is reachable by reading words generated from $q$ in $\mathcal{N}_{1} .{ }^{1}$ Clearly, the inclusion $L_{1} \subseteq L_{2}$ holds iff none of the sets of states computed for the initial states of $\mathcal{N}_{1}$ contain some initial state of $\mathcal{N}_{2}$.

In order to prevent the computation of all possible subsets of $\mathcal{N}_{2}$ from which the final states are non-reachable, which would be equivalent to determinizing $\mathcal{N}_{2}$, the antichains algorithm ensures that the set $S_{q}$ for each state $q$ in $\mathcal{N}_{1}$ is an antichain, i.e. $\forall s, s^{\prime} \in S_{q}, s \nsubseteq s^{\prime} \wedge s^{\prime} \nsubseteq S$. The idea behind the use of antichains is that, given two sets of states of $\mathcal{N}_{2}$, namely $s$ and $s^{\prime}$, if $s \subseteq s^{\prime}$ then if no final state of $\mathcal{N}_{2}$ is reachable from $s^{\prime}$ by reading words in a certain set then the same holds for $s$. Therefore, discarding the set $s$ and keeping the set $s^{\prime}$ preserves the correctness of the algorithm. The resulting algorithm is refer to as the backward antichains algorithm.

\footnotetext{
${ }^{1}$ Note that this is equivalent to to finding states of the complement of the determinized version of $\mathcal{N}_{2}$ from which a final state is reachable by reading a word generated from $q$ in $\mathcal{N}_{1}$.
} 
Furthermore, Wulf et al. [2006] also defined a dual of the antichains algorithm described above. In this case, the algorithm computes the set $\widetilde{S}_{q}$ of sets of states of $\mathcal{N}_{2}$ reachable from an initial state by reading a word generated from $q$ in $\mathcal{N}_{1}$. In this case, the inclusion $L_{1} \subseteq L_{2}$ holds iff for every initial state $q$ of $\mathcal{N}_{1}$, all the sets in $\widetilde{S}_{q}$ contain a final state. Again, by ensuring that $\widetilde{S}_{q}$ is an antichain, we can reduce the number of sets of states of $\mathcal{N}_{2}$ that need to be computed since, whenever $s \subseteq s^{\prime}$, if a final state is reachable from $s$ by a word in a given language, the same holds for $s^{\prime}$ and, therefore, it is possible to discard $s^{\prime}$. The resulting algorithm is refer to as the forward antichains algorithm.

The proof of the correctness of the antichains algorithm, as presented by Wulf et al. [2006], heavily depends on the automata representation of the languages. We believe that our quasiorder-based framework, presented in Chapter 4, offers a better understanding on the antichains algorithm and its correctness proof by offering a new explanation of the algorithm from a language perspective.

\section{Improvements on the Antichains Algorithm}

The antichains algorithm of Wulf et al. [2006] was later improved by Abdulla et al. [2010], who used simulations (between states and between sets of states) for reducing the amount of sets of states considered by the algorithm.

In particular, they found that, for the forward antichains algorithm, there is no need to add the set $s$ of states of $\mathcal{N}_{2}$ to the set $\widetilde{S}_{q}$ for a certain state $q$ of $\mathcal{N}_{1}$ if there exists a state $q^{\prime}$ of $\mathcal{N}_{1}$ such that $q$ simulates $q^{\prime}$ and whose associated set $\widetilde{S}_{q^{\prime}}$ contains a set $s^{\prime}$ that simulates $s$. The idea behind this approach is that simulation is a sufficient condition for language inclusion to hold, i.e. if the set of states $s^{\prime}$ simulates the set $s$ then the language generated from $s^{\prime}$ is a subset of the language generated from $s$.

As we show in Chapter 4, this improvement on the antichains algorithm can be partially accommodated by our quasiorder-based framework by using simulations in the definition of the quasiorder. By doing so, the resulting algorithm matches the behavior of the one of Abdulla et al. [2010] when $q=q^{\prime}$.

On the other hand, Bonchi and Pous [2013] defined a new type of relation between sets of states, denoted bisimulation up to congruence, and used it to define a new algorithm for deciding language equivalence between sets of states of a given automaton.

Intuitively, bisimulations up to congruence are enhanced bisimulations (and, therefore, if they relate two sets of states then both sets generate the same language) that might relate sets of states that are not explicitly related by the underlying bisimulation but are related by its implicit congruence closure. Since $L_{1} \subseteq L_{2} \Leftrightarrow L_{1} \cup L_{2}=L_{2}$, the algorithm of Bonchi and Pous [2013] can be used to 
decide the inclusion $L_{1} \subseteq L_{2}$ by considering the union automaton $\mathcal{N}_{1} \cup \mathcal{N}_{2}$ and checking whether the bisimulations up to congruence holds between the union of the initial states of $\mathcal{N}_{1}$ and $\mathcal{N}_{2}$, which generate $L_{1} \cup L_{2}$, and the initial states of $\mathcal{N}_{2}$, which generate $L_{2}$.

Finally, Holík and Meyer [2015] used antichains to solve the language inclusion problem $L_{1} \subseteq L_{2}$ when $L_{1}$ is a context-free language and $L_{2}$ is regular. To do that, they reduced the language inclusion problem to a data flow analysis one. This allowed them to rephrase the language inclusion problem as an inclusion problem between sets of relations on the states of the automaton. Then, they applied the antichains principle to reduce the number of relations that need to be manipulated.

As we show in Chapter 4, our quasiorder-based framework for deciding the language inclusion $L_{1} \subseteq L_{2}$ also applies to the case in which $L_{1}$ is a context-free grammar. Indeed, when $L_{1}$ is regular we instantiate our framework with left or right monotone quasiorders and obtain the antichains algorithm of Wulf et al. [2006] and its variants, among other algorithms. Similarly, when $L_{1}$ is contextfree, we use a left and right monotone quasiorders and obtain the antichains algorithm of Holík and Meyer [2015], among others.

Therefore, our framework allows us to offer a more direct presentation of the antichains algorithm for grammars of Holík and Meyer [2015] as an straightforward extension of the antichains algorithm for regular languages.

\subsubsection{Solving Language Inclusion through Abstractions}

Our approach draws inspiration from the work of Hofmann and Chen [2014], who considered the language inclusion problem on infinite words $L_{1} \subseteq L_{2}$ where $L_{1}$ is represented by a Büchi automata and $L_{2}$ is regular.

They defined a language inclusion algorithm based on fixpoint computations and a language abstraction based on an equivalence relation between states of the underlying automata representation. Although the equivalence relation is folklore (you find it in several textbooks on language theory [Khoussainov and Nerode 2001; Sakarovitch 2009]), Hofmann and Chen [2014] were the first, to the best of our knowledge, to use it as an abstraction and, in particular, as a complete domain in abstract interpretation.

As we show in Chapter 4, our framework for solving the language inclusion problem also relies on computing the language abstraction of a fixpoint computation. However, we focus on languages on finite words and generalize the language abstractions by relaxing their equivalence relations to quasiorders. Moreover, by considering quasiorders instead of equivalences, we are able to generalize the fixed point-based approach to check $L_{1} \subseteq L_{2}$ when $L_{2}$ is non-regular. 


\subsection{Searching on Compressed Text}

The problem of searching with regular expressions on grammar-compressed text has been extensively studied for the last decades. Results in this topic can be divided in two main groups:

a) Characterization of the problem's complexity from a theoretical point of view [Plandowski and Rytter 1999; Markey and Schnoebelen 2004; Abboud et al. 2017].

b) Development of algorithms and data structures to efficiently solve different versions of the problem such as pattern matching [Navarro and Tarhio 2005; de Moura et al. 1998; Mäkinen and Navarro 2006], approximate pattern matching [Bille et al. 2009; Kärkkäinen et al. 2003], multipattern matching [Kida et al. 1998; Gawrychowski 2014], regular expression matching [Navarro 2003; Bille et al. 2009] and subsequence matching [Bille et al. 2014].

To characterize the complexity of search problems on grammar-compressed text it is common to use straight line programs (grammars generating a single string) to represent the output of the compression. Straight line programs are a natural model for algorithms such as LZ78 [Ziv and Lempel 1978], LZW [Welch 1984], Recursive Pairing [Larsson and Moffat 1999] or Sequitur [Nevill-Manning and Witten 1997] and, as proven by Rytter [2004], polynomially equivalent to LZ77 [Ziv and Lempel 1977].

However, algorithms for searching with regular expressions on grammarcompressed text are typically designed for a particular compression scheme [Navarro and Tarhio 2005; Navarro 2003; Bille et al. 2009].

The first algorithm to solve this problem is due to Navarro [2003] and it is defined for LZ78/LZW compressed text. His algorithm reports all positions in the uncompressed text at which a substring that matches the expression ends and exhibits $O\left(2^{s}+s \cdot T+\mathrm{occ} \cdot s \cdot \log s\right)$ worst case time complexity using $O\left(2^{s}+t \cdot s\right)$ space, where "occ" is the number of occurrences, $s$ is the size of the expression and $T$ is the length of the text compressed to size $t$. To the best of our knowledge this is the only algorithm for regular expression searching on compressed text that has been implemented and evaluated in practice.

Bille et al. [2009] improved the result of Navarro by defining a relationship between the time and space required to perform regular expression searching on compressed text. They defined a data structure of size $o(t)$ to represent LZ78 compressed texts and an algorithm that, given a parameter $\tau$, finds all occurrences of a regular expression in a LZ78 compressed text in $O(t \cdot s \cdot(s+\tau)+$ occ $\cdot s \cdot \log s)$ time using $O\left(t \cdot s^{2} / \tau+t \cdot s\right)$ space. To the best of our knowledge, no implementation 
of this algorithm was carried out.

We tackle the problem of searching in grammar-compressed text by using our algorithms for deciding language inclusion adapted to efficiently handle straight line programs and enhanced with additional information that is computed for each variable of the grammar in order to find the exact matches.

Our approach, presented in Chapter 5, differs from the previous ones in the generality of its definition since, by working on straight line programs, our algorithm and its complexity analysis apply to any grammar-based compression scheme. This is a major improvement since, as shown by Hucke et al. [2016], the LZ78 representation of a text of length $T$ has size $t=\Theta\left((T / \log (T))^{2 / 3}\right)$ while its representation as a straight line program has size $t=\Omega(\log (T) /(\log \log (T)))$ and $t=O\left((T / \log (T))^{2 / 3}\right)$. Therefore, our approach allows us to handle much more concise representations of the data.

Moreover, the definition of "occurrence" used in previous works, i.e. positions in the uncompressed text from which we can read a substring matching the expression, is of limited practical interest. As an evidence, state of the art tools for regular expression searching, such as grep or ripgrep, define an occurrence as a line of text containing a match of the expression and so do us.

As a consequence, our algorithm reports the number of occurrences of a fixed regular expression in a compressed text in $O(t)$ time while previous algorithms require $O(T)$ since occ $=O(T)$. Even when there are no matches (occ $=0$ ), so previous approaches operate in $O(t)$ time, the result of Hucke et al. [2016] shows that our algorithm behaves potentially better than the others.

\section{Deciding the Existence of a Match}

It is worth to remark that the problem of deciding language inclusion between the languages generated by a straight line program and an automaton has been studied before. In particular Plandowski and Rytter [1999] reduced this problem to a series of matrix multiplications, showing that it can be solved in $O\left(t \cdot s^{3}\right)$ time $(O(t \cdot s)$ for deterministic automata) where $t$ is the size of the grammar and $s$ is the size of the automaton. Note that this problem corresponds to deciding whether a grammar-compressed text contains a match for a given regular expression.

On the other hand, Esparza et al. [2000] defined an algorithm to solve a number of decision problems involving automata and context-free grammars which, when restricted to grammars generating a single word, results in a particular implementation of Plandowsky's approach. Indeed, this implementation coincides with our Algorithm SLPINCS, presented in Chapter 5 as a straightforward adaptation of the algorithm presented in Chapter 4 for deciding the inclusion of a context-free language into a regular one. 


\subsection{Building Residual Automata}

Residual automata (RFA for short) were first introduced by Denis et al. [2000; 2001; 2002]. We deliberatively use the notation RFA for residual automata, instead of the standard RFSA, in order to be consistent with the notation used in this thesis for deterministic (DFA) and non-deterministic (NFA) automata.

When introducing RFAs, Denis et al. [2000] also defined an algorithm for residualizing an automaton, which is an adaptation of the well-known subset construction used for determinization. Moreover, they showed that there exists a unique canonical RFA, which is minimal in number of states, for every regular language. Finally, they showed that the residual-equivalent of the doublereversal method holds, i.e. residualizing an automaton $\mathcal{N}$ whose reverse is residual yields the canonical RFA for the language generated by $\mathcal{N}$.

Later, Tamm [2015] generalized the double-reversal method for RFAs by giving a sufficient and necessary condition that guarantees that the residualization operation defined by Denis et al. [2002] yields the canonical RFA. This generalization comes in the same lines as that of Brzozowski and Tamm [2014] for the double-reversal method for DFAs.

In Chapter 6, we present a quasiorder-based framework of automata constructions inspired by the work of Ganty et al. [2019], who defined a framework of automata constructions based on equivalences over words to provide new insights on the relation between well-known methods for computing the minimal deterministic automaton of a language. Intuitively, the shift from equivalences to quasiorders allows us to move from deterministic automata to residual ones.

In their work, Ganty et al. [2019] used congruences, i.e. monotone equivalences, over words that induce finite partitions over $\Sigma^{*}$. Then, they used wellknown automata constructions that yield automata generating a given language $L$ [Büchi 1989; Khoussainov and Nerode 2001] to derive new automata constructions parametrized by a congruence. As a result, when using the Nerode's congruence for $L$, their automata construction yields the minimal DFA for $L$ [Büchi 1989; Khoussainov and Nerode 2001] while, when using the so-called automatabased equivalence relative to an NFA their construction yields the determinized version of the input NFA. They also obtained counterpart automata constructions that yield, respectively, the minimal co-deterministic and a co-deterministic automaton for the language.

The relation between the automata constructions resulting from the Nerode's and the automata-based congruences allowed them to relate determinization and minimization operations. Finally, they re-formulated the generalization of the double-reversal method presented by Brzozowski and Tamm [2014], which gives a sufficient and necessary condition that guarantees that determinizing an NFA 
yields the minimal DFA for the language generated by the NFA.

Our quasiorder-based framework allows us to extend the work of Ganty et al. [2019] and devise automata constructions that result in residual automata. Moreover, we derive a residual-equivalent of the generalized double-reversal method from Brzozowski and Tamm [2014] that is more general than the one presented by Tamm [2015]. 


\section{BACKGROUND}

In this section we introduce all the concepts and notation that will be used throughout the rest of the thesis.

\subsection{Words and Languages}

Let $\Sigma$ be a finite nonempty alphabet of symbols. A string or word $w$ is a finite sequence of symbols of $\Sigma$ where the empty sequence is denoted $\varepsilon$. We denote $w^{R}$ the reverse of $w$ and use $|w|$ to denote the length of $w$ that we abbreviate to $\dagger$ when $w$ is clear from the context. We define $(w)_{i}$ as the $i$-th symbol of $w$ if $1 \leq i \leq \dagger$ and $\varepsilon$ otherwise. Similarly, $(w)_{i, j}$ denotes the substring, also called factor, of $w$ between the $i$-th and the $j$-th symbols, both included. Clearly, $w=(w)_{1, \uparrow}$.

We write $\Sigma^{*}$ to denote the set of all finite words on $\Sigma$ and write $\wp(S)$ to denote the set of all subsets of $S$, i.e. $\wp(S) \stackrel{\text { def }}{=}\left\{S^{\prime} \mid S^{\prime} \subseteq S\right\}$. Given a language $L \in \wp\left(\Sigma^{*}\right)$, $L^{R} \stackrel{\text { def }}{=}\left\{w^{R} \mid w \in L\right\}$ denotes the reverse of $L$ while $L^{c} \stackrel{\text { def }}{=}\left\{w \in \Sigma^{*} \mid w \notin L\right\}$ denotes its complement. Concatenation in $\Sigma^{*}$ is simply denoted by juxtaposition, both for concatenating words $u v$, languages $L_{1} L_{2}$ and words with languages such as $u L v$. We sometimes use the symbol - to refer explicitly to a concatenation operation.

Definition (Quotient). Let $L \subseteq \Sigma^{*}$ and $u \in \Sigma^{*}$. The left quotient of $L$ by the word $u$ is the set of suffixes of the word $u$ in $L$, i.e.

$$
u^{-1} L \stackrel{\text { def }}{=}\left\{w \in \Sigma^{*} \mid u w \in L\right\} .
$$

Similarly, the right quotient of $L$ by the word $u$ is the set of all prefixes of $u$ in $L$, i.e.

$$
L u^{-1} \stackrel{\text { def }}{=}\left\{w \in \Sigma^{*} \mid w u \in L\right\} .
$$


Finally, we lift the notions of left and right quotients by a word to sets $S \subseteq \Sigma^{*}$ as:

$$
S^{-1} L \stackrel{\text { def }}{=}\left\{w \in \Sigma^{*} \mid \forall s \in S, s w \in L\right\} \text { and } L S^{-1} \stackrel{\text { def }}{=}\left\{w \in \Sigma^{*} \mid \forall s \in S, w s \in L\right\}
$$

Note that the definition of quotient by a set is unconventional as it uses the universal quantifier instead of existential. We use this definition since it guarantees that the quotient by a set is the adjoint of concatenation, i.e.

$$
X Y \subseteq L \Leftrightarrow Y \subseteq X^{-1} L \Leftrightarrow X \subseteq L Y^{-1} .
$$

Definition (Composite and Prime Quotients). A left (resp. right) quotient $u^{-1} L$ is composite iff it is the union of all the left (resp. right) quotients that it strictly contains, i.e.

$$
\left.u^{-1} L=\bigcup_{x \in \Sigma^{*}, x^{-1} L \subseteq u^{-1} L} x^{-1} L \quad \text { (resp. } L u^{-1}=\bigcup_{x \in \Sigma^{*}, L x^{-1} \subsetneq L u^{-1}} L x^{-1}\right) .
$$

When a quotient is not composite, we say it is prime.

\subsection{Finite-state Automata}

Throughout this dissertation we consider three different classes of automata: non-deterministic, deterministic and residual. Next, we define these classes of automata and introduce some basic notions related them.

\section{Non-Deterministic Finite-State Automata}

Definition (NFA). A non-deterministic finite-state automaton (NFA for short) is a tuple $\mathcal{N}=\langle Q, \Sigma, \delta, I, F\rangle$ where $\Sigma$ is the alphabet, $Q$ is the finite set of states, $I \subseteq Q$ is the subset of initial states, $F \subseteq Q$ is the subset of final states, and $\delta: Q \times \Sigma \rightarrow \wp(Q)$ is the transition relation.

We sometimes use the notation $q \stackrel{a}{\rightarrow} q^{\prime}$ to denote that $q^{\prime} \in \delta(q, a)$. If $u \in \Sigma^{*}$ and $q, q^{\prime} \in Q$ then $q \stackrel{u}{\sim} q^{\prime}$ means that the state $q^{\prime}$ is reachable from $q$ by following the string $u$. Formally, by induction on the length of $u \in \Sigma^{*}$ :

(i) if $u=\epsilon$ then $q \stackrel{\epsilon}{\sim} q^{\prime}$ iff $q=q^{\prime}$;

(ii) if $u=a v$ with $a \in \Sigma, v \in \Sigma^{*}$ then $q \stackrel{a v}{\sim} q^{\prime}$ iff $\exists q^{\prime \prime} \in \delta(q, a), q^{\prime \prime} \stackrel{v}{\sim} q^{\prime}$.

The language generated by an NFA $\mathcal{N}$, often referred to as the language accepted by $\mathcal{N}$ is $\mathcal{L}(\mathcal{N}) \stackrel{\text { def }}{=}\left\{u \in \Sigma^{*} \mid \exists q_{i} \in I, \exists q_{f} \in F, q_{i} \stackrel{u}{\rightarrow} q_{f}\right\}$. We define the successors and the predecessors of a set $S \subseteq Q$ by a word $w \in \Sigma^{*}$ as:

$$
\operatorname{post}_{w}^{\mathcal{N}}(S) \stackrel{\text { def }}{=}\left\{q \in Q \mid \exists q^{\prime} \in S, q^{\prime} \stackrel{w}{\sim} u\right\}
$$




$$
\operatorname{pre}_{w}^{\mathcal{N}}(S) \stackrel{\text { def }}{=}\left\{q \in Q \mid \exists q^{\prime} \in S, q \stackrel{w}{\rightarrow} q^{\prime}\right\}
$$

In general, we omit the automaton $\mathcal{N}$ from the superscript when it is clear from the context. Figure 3.1 shows an example of an NFA.

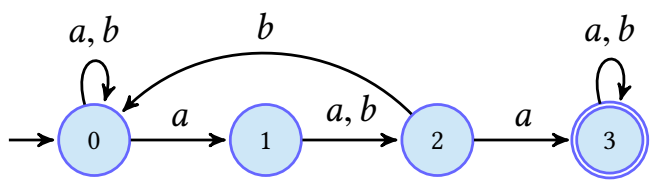

Figure 3.1: An NFA $\mathcal{N}$ with $\Sigma=\{a, b\}$ and $\mathcal{L}(\mathcal{N})=\Sigma^{*} a \Sigma a \Sigma^{*}$.

Given $S, T \subseteq Q$, define

$$
\left.W_{S, T}^{\mathcal{N}} \stackrel{\text { def }}{=}\left\{w \in \Sigma^{*} \mid \exists q \in S, q^{\prime} \in T, q \stackrel{w}{\sim} q^{\prime}\right)\right\}
$$

When $S$ or $T$ are singletons, we abuse of notation and write $W_{q, T}^{\mathcal{N}}, W_{S, q^{\prime}}^{\mathcal{N}}$ or even $W_{q, q^{\prime}}^{\mathcal{N}}$. In particular, when $S=\{q\}$ and $T=F$, we say that $W_{q, F}^{\mathcal{N}}$ is the right language of $q$. Likewise, when $S=I$ and $T=\{q\}$, we say that $W_{I, q}^{\mathcal{N}}$ is the left language of $q$. We say that a state $q$ is unreachable iff $W_{I, q}^{\mathcal{N}}=\varnothing$ and we say that $q$ is empty iff $W_{q, F}^{\mathcal{N}}=\varnothing$. Finally, note that

$$
\mathcal{L}(\mathcal{N})=\bigcup_{q \in I} W_{q, F}^{\mathcal{N}}=\bigcup_{q \in F} W_{I, q}^{\mathcal{N}}=W_{I, F}^{\mathcal{N}}
$$

Definition (Sub-automaton). Let $\mathcal{N}=\langle Q, \Sigma, \delta, I, F\rangle$ be an NFA. A sub-automaton of $\mathcal{N}$ is an $N F A \mathcal{N}^{\prime}=\left\langle Q^{\prime}, \Sigma, \delta^{\prime}, I^{\prime}, F^{\prime}\right\rangle$ for which $Q^{\prime} \subseteq Q, F^{\prime} \subseteq F, I^{\prime} \subseteq I$ and for every $q, q^{\prime} \in Q$ and $a \in \Sigma$ we have that $q^{\prime} \in \delta^{\prime}(q, a) \Rightarrow q^{\prime} \in \delta(q, a)$.

Clearly, if $\mathcal{N}^{\prime}$ is a sub-automaton of $\mathcal{N}$ then $\mathcal{L}\left(\mathcal{N}^{\prime}\right) \subseteq \mathcal{L}(\mathcal{N})$.

Definition (Reverse Automaton). Let $\mathcal{N}=\langle Q, \Sigma, \delta, I, F\rangle$ be an NFA. The reverse of $\mathcal{N}$ is the $N F A \mathcal{N}^{R} \stackrel{\text { def }}{=}\left\langle Q, \Sigma, \delta^{R}, F, I\right\rangle$ where for every $q, q^{\prime} \in Q$ and $a \in \Sigma$ we have that $q \in \delta^{R}\left(q^{\prime}, a\right)$ iff $q^{\prime} \in \delta(q, a)$.

It is straightforward to check that $\mathcal{L}(\mathcal{N})^{R}=\mathcal{L}\left(\mathcal{N}^{R}\right)$.

\section{Deterministic Finite-State Automata}

Definition (DFA and co-DFA). A deterministic finite-state automaton (DFA for short) is an NFA such that $I=\left\{q_{0}\right\}$ and, for every state $q \in Q$ and every symbol $a \in \Sigma$, there exists at most one state $q^{\prime} \in Q$ such that $\delta(q, a)=q^{\prime}$. 
A co-deterministic finite-state automaton (co-DFA for short) is an NFA $\mathcal{N}$ such that $\mathcal{N}^{R}$ is deterministic.

Definition (Subset Construction). Let $\mathcal{N}=\langle Q, \Sigma, \delta, I, F\rangle$ be an NFA. The subset construction builds a DFA $\mathcal{N}^{D} \stackrel{\text { def }}{=}\left\langle Q^{D}, \Sigma, \delta^{D}, I^{D}, F^{D}\right\rangle$ where

$$
\begin{aligned}
Q^{D} & \stackrel{\text { def }}{=}\left\{\operatorname{post}_{u}^{\mathcal{N}}(I) \mid u \in \Sigma^{*}\right\} \\
I^{D} & \stackrel{\text { def }}{=}\{I\} \\
F^{D} & \stackrel{\text { def }}{=}\{S \in \wp(Q) \mid S \cap F \neq \varnothing\} \\
\delta^{D}(S, a) & \stackrel{\text { def }}{=}\left\{q^{\prime} \mid \exists q \in S, q^{\prime} \in \delta(q, a)\right\} \text { for every } S \in Q \text { and } a \in \Sigma
\end{aligned}
$$

Given an NFA $\mathcal{N}$, we denote by $\mathcal{N}^{D}$ the DFA that results from applying the subset construction to $\mathcal{N}$ where only subsets that are reachable from the initial states of $\mathcal{N}^{D}$ are used. As shown by Hopcroft et al. [2001], $\mathcal{L}\left(\mathcal{N}^{D}\right)=\mathcal{L}(\mathcal{N})$ for every automaton $\mathcal{N}$. Figure 3.2 shows the DFA obtained when applying the subset construction to the NFA from Figure 3.1.

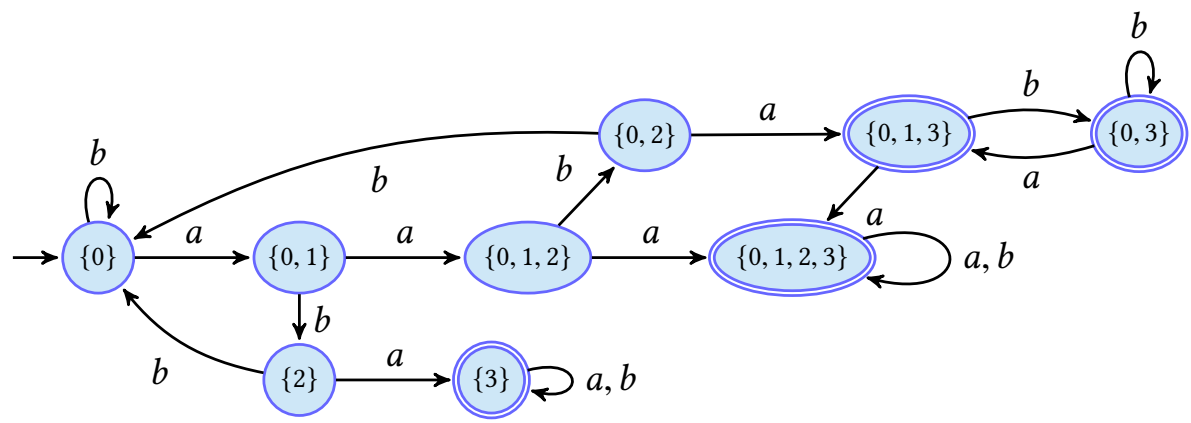

Figure 3.2: DFA $\mathcal{N}^{D}$ obtained by determinizing the NFA from Figure 3.1.

A DFA for the language $\mathcal{L}(\mathcal{N})$ is minimal, denoted by $\mathcal{N}^{D M}$, if it has no unreachable states and no two states have the same right language. For instance, the DFA from Figure 3.2 is not minimal since the states $\{0,1,3\},\{0,3\},\{0,1,2,3\}$ and $\{3\}$ all have the same right language. The minimal DFA for a regular language is unique modulo isomorphism and is determined by the right quotients of the generated language.

Definition (Minimal DFA). Let $L$ be a regular language. The minimal DFA for $L$ is the DFA $\mathcal{D} \stackrel{\text { def }}{=}\left\langle Q^{D}, \Sigma, \delta^{D}, I^{D}, F^{D}\right\rangle$ where

$$
Q^{D} \stackrel{\text { def }}{=}\left\{u^{-1} L \mid u \in \Sigma^{*}\right\}
$$




$$
\begin{aligned}
I^{D} & \stackrel{\text { def }}{=}\left\{u^{-1} L \in Q \mid u^{-1} L \subseteq L\right\} \\
F^{D} & \stackrel{\text { def }}{=}\left\{u^{-1} L \in Q \mid \varepsilon \in u^{-1} L\right\} \\
\delta^{D}\left(u^{-1} L, a\right) & \stackrel{\text { def }}{=}\left\{v^{-1} L \in Q \mid v^{-1} L=a^{-1}\left(u^{-1} L\right)\right\} \text { for every } u^{-1} L \in Q \text { and } a \in \Sigma
\end{aligned}
$$

\section{Residual Finite-State Automata}

Definition (RFA and co-RFA). A residual finite-state automaton (RFA for short) is an NFA such that the right language of each state is a left quotient of the language generated by the automaton.

A co-residual automaton (co-RFA for short) is an NFA $\mathcal{N}$ such that $\mathcal{N}^{R}$ is residual, i.e. the left language of each state is a right quotient of the language generated by the automaton.

Formally, an RFA is an NFA $\mathcal{N}=\langle Q, \Sigma, \delta, I, F\rangle$ satisfying

$$
\forall q \in Q, \exists u \in \Sigma^{*}, W_{q, F}=u^{-1} \mathcal{L}(\mathcal{N}) .
$$

Similarly, $\mathcal{N}$ is a co-RFA iff it satisfies

$$
\forall q \in Q, \exists u \in \Sigma^{*}, W_{I, q}=L u^{-1} .
$$

The right quotients of the form $u^{-1} L$, where $L \subseteq \Sigma^{*}$ is a language and $u \in \Sigma^{*}$, are also known as residuals, which gives name to RFAs. We say $u \in \Sigma^{*}$ is a characterizing word for $q \in Q$ iff $W_{q, F}^{\mathcal{N}}=u^{-1} \mathcal{L}(\mathcal{N})$ and we say $\mathcal{N}$ is consistent iff each state $q$ is reachable by a characterizing word for $q$. Moreover, $\mathcal{N}$ is strongly consistent iff every state $q$ is reachable by every characterizing word of $q$.

Similarly to the case of DFAs, there exists a residualization operation [Denis et al. 2002] that, given an NFA $\mathcal{N}$, builds an RFA $\mathcal{N}^{\text {res }}$ such that $\mathcal{L}\left(\mathcal{N}^{\text {res }}\right)=\mathcal{L}(\mathcal{N})$. This construction can be seen as a determinization followed by the removal of coverable states and the addition of new transitions. We say that the set $\operatorname{post}_{u}^{\mathcal{N}}(I)$ is coverable iff

$$
\operatorname{post}_{u}^{\mathcal{N}}(I)=\bigcup_{x \in \Sigma^{*}, \operatorname{post}_{x}^{\mathcal{N}}(I) \subseteq \operatorname{post}_{u}^{\mathcal{N}}(I)} \operatorname{post}_{x}^{\mathcal{N}}(I)
$$

Definition (Residualization). Let $\mathcal{N}=\langle Q, \Sigma, \delta, I, F\rangle$ be an NFA. Then the residualization operation builds the $R F A \mathcal{N}^{\text {res }} \stackrel{\text { def }}{=}\langle\widetilde{Q}, \Sigma, \widetilde{\delta}, \widetilde{I}, \widetilde{F}\rangle$ with

$$
\begin{aligned}
\widetilde{Q} & \stackrel{\text { def }}{=}\left\{\operatorname{post}_{u}^{\mathcal{N}}(I) \mid u \in \Sigma^{*} \wedge \operatorname{post}_{u}^{\mathcal{N}}(I) \text { is not coverable }\right\} \\
\widetilde{I} & \stackrel{\text { def }}{=}\{S \in \widetilde{Q} \mid S \subseteq I\} \\
\widetilde{F} & \stackrel{\text { def }}{=}\{S \in \widetilde{Q} \mid S \cap F \neq \varnothing\} \\
\widetilde{\delta}(S, a) & =\left\{S^{\prime} \in \widetilde{Q} \mid S^{\prime} \subseteq \delta(S, a)\right\} \text { for every } S \in \widetilde{Q} \text { and } a \in \Sigma
\end{aligned}
$$


Figure 3.3 shows the RFA obtained by applying the residualization operation to the NFA from Figure 3.1.

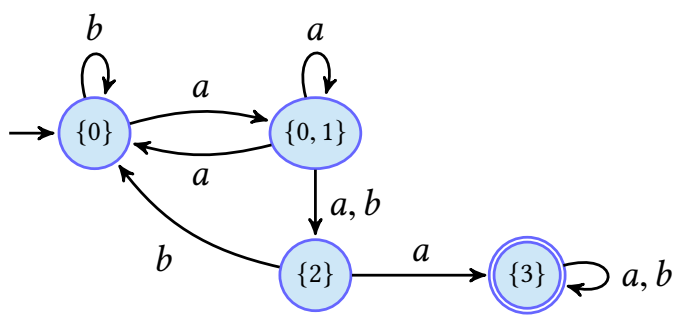

Figure 3.3: $R F A \mathcal{N}^{\text {res }}$ obtained when residualizing to the NFA from Figure 3.1.

Similarly, to the case of DFAs, there exists an RFA for every regular language that is minimal in the number of states and is unique modulo isomorphism: the canonical RFA.

Definition (Canonical RFA). Let $L$ be a regular language. The canonical RFA for $L$ is the RFA $C \stackrel{\text { def }}{=}\left\langle Q^{C}, \Sigma, \delta^{C}, I^{C}, F^{C}\right\rangle$ with

$$
\begin{aligned}
Q^{C} & \stackrel{\text { def }}{=}\left\{u^{-1} L \mid u \in \Sigma^{*}, u^{-1} L \text { is prime }\right\} \\
I^{C} & \stackrel{\text { def }}{=}\left\{u^{-1} L \in Q \mid u^{-1} L \subseteq L\right\} \\
F^{C} & \stackrel{\text { def }}{=}\left\{u^{-1} L \in Q \mid \varepsilon \in u^{-1} L\right\} \\
\delta^{C}\left(u^{-1} L, a\right) & \stackrel{\text { def }}{=}\left\{v^{-1} L \in Q \mid v^{-1} L \subseteq a^{-1}\left(u^{-1} L\right)\right\} \text { for every } u^{-1} L \in Q \text { and } a \in \Sigma
\end{aligned}
$$

The canonical RFA is a strongly consistent RFA and it is the minimal (in number of states) RFA such that $\mathcal{L}(C)=L$ [Denis et al. 2002]. Moreover, by definition, the canonical RFA has the maximal number of transitions.

Finally, it is straightforward to check that any DFA $\mathcal{D}$ is also an RFA since $W_{q, F}^{\mathcal{D}}=u^{-1} L$ for all $u \in W_{I, q}^{\mathcal{D}}$. Therefore, we have the following relations between these classes of automata:

$$
\mathrm{DFA} \subsetneq \mathrm{RFA} \subsetneq \mathrm{NFA}
$$

\subsection{Context-free Grammars}

Definition (CFG). A context-free grammar (grammar or CFG for short) is a tuple $\mathcal{G} \stackrel{\text { def }}{=}\langle\mathcal{V}, \Sigma, P\rangle$ where $\mathcal{V}=\left\{X_{0}, \ldots, X_{n}\right\}$ is a finite set of variables including the start symbol $X_{0}$ (also denoted axiom), $\Sigma$ is a finite alphabet of terminals and $P$ is the set of rules $X_{i} \rightarrow \beta$ where $\beta \in(\mathcal{V} \cup \Sigma)^{*}$ 
In the following we assume, for simplicity and without loss of generality, that grammars are always given in Chomsky Normal Form (CNF) [Chomsky 1959], that is, every rule $X_{i} \rightarrow \beta \in P$ is such that $\beta \in(\mathcal{V} \times \mathcal{V}) \cup \Sigma \cup\{\epsilon\}$ and if $\beta=\epsilon$ then $i=0$. We also assume that for all $X_{i} \in \mathcal{V}$ there exists a rule $X_{i} \rightarrow \beta \in P$, otherwise $X_{i}$ can be safely removed from $\mathcal{V}$.

Given two strings $w, w^{\prime} \in(\mathcal{V} \cup \Sigma)^{*}$ we write $w \Rightarrow w^{\prime}$ iff there exists two strings $u, v \in(\mathcal{V} \cup \Sigma)^{*}$ and a grammar rule $X \rightarrow \beta \in P$ such that $w=u X v$ and $w^{\prime}=u \beta v$. We denote by $\Rightarrow^{*}$ the reflexive-transitive closure of $\Rightarrow$.

The language generated by a $\mathcal{G}$ is $\mathcal{L}(\mathcal{G}) \stackrel{\text { def }}{=}\left\{w \in \Sigma^{*} \mid X_{0} \Rightarrow^{*} w\right\}$.

\section{Straight-line Programs}

In the context of grammar-based compression we are interested in straight line programs, i.e. grammars generating exactly one word.

Definition (SLP). A straight line program (SLP for short), is a $C F G \mathcal{P}=\langle\mathcal{V}, \Sigma, P\rangle$ where the set of rules is of the form

$$
P \stackrel{\text { def }}{=}\left\{X_{i} \rightarrow \alpha_{i} \beta_{i}|1 \leqslant i \leqslant| V \mid, \alpha_{i}, \beta_{i} \in\left(\Sigma \cup\left\{X_{1}, \ldots, X_{i-1}\right\}\right\} .\right.
$$

We refer to $X_{|V|} \rightarrow \alpha_{|V|} \beta_{|V|}$ as the axiom rule.

It is straightforward to check that the language generated by an SLP consists of a single string $w \in \Sigma^{*}$ and, by definition, $|w|>1$. Since $\mathcal{L}(P)=\{w\}$ we identify $w$ with $\mathcal{L}(P)$.

\subsection{Quasiorders}

Let $f: X \rightarrow Y$ be a function between sets and let $S \in \wp(X)$. We denote the image of $f$ on $S$ by $f(S) \stackrel{\text { def }}{=}\{f(x) \in Y \mid x \in S\}$. The composition of two functions $f$ and $g$ is denoted both by $f g$ and $f \circ g$.

A quasiordered set (qoset for short) is a tuple $\langle D, \leqslant\rangle$ such that $\leqslant$ is a quasiorder (qo for short) relation on $D$, i.e. a reflexive and transitive binary relation. Given a qoset $\langle D, \leqslant\rangle$ we denote by $\sim_{D}$ the equivalence relation induced by $\leqslant$ :

$$
d \sim_{D} d^{\prime} \stackrel{\text { def }}{\Leftrightarrow} d \leqslant d^{\prime} \wedge d^{\prime} \leqslant d, \quad \text { for all } d, d^{\prime} \in D .
$$

Moreover, given a qo $\leqslant$ we denote its strict version by $<$ :

$$
u<v \stackrel{\text { def }}{\Leftrightarrow} u \leqslant v \wedge v \nless u .
$$


We say that a qoset satisfies the ascending (resp. descending) chain condition (ACC, resp. DCC) if there is no countably infinite sequence of distinct elements $\left\{x_{i}\right\}_{i \in \mathbb{N}}$ such that, for all $i \in \mathbb{N}, x_{i} \leqslant x_{i+1}$ (resp. $x_{i+1} \leqslant x_{i}$ ). If a qoset satisfies the ACC (resp. DCC) we say it is ACC (resp. DCC).

Definition (Closure and Principals). Let $\leqslant$ be a quasiorder on $\Sigma^{*}$ and let $S \subseteq \Sigma^{*}$. The closure of $S$ is the set

$$
\rho_{\leqslant}(S) \stackrel{\text { def }}{=}\left\{w \in \Sigma^{*} \mid \exists x \in S, x \leqslant w\right\} .
$$

We say $\rho_{\leqslant}(S)$ is a principal if $S$ is a singleton. In that case, we abuse of notation and write $\rho_{\leqslant}(u)$ instead of $\rho_{\leqslant}(\{u\})$.

Given two quasiorders $\leqslant$ and $\leqslant^{\prime}$ we say that $\leqslant$ is finer than $\leqslant^{\prime}$ (or $\leqslant^{\prime}$ is coarser than $\leqslant$ ) and write $\leqslant \subseteq \leqslant^{\prime}$ iff $\rho_{\leqslant}(S) \subseteq \rho_{\leqslant^{\prime}}(S)$ for every set $S \subseteq \Sigma^{*}$.

Definition (Left and Right Quasiorders). Let $\leqslant$ be a quasiorder. We say $\leqslant i$ ig right monotone (or equivalently, $\leqslant$ is a right quasiorder), and denote it $b y \leqslant{ }^{r}$, iff

$$
u \leqslant^{r} v \Rightarrow u a \leqslant^{r} v a, \quad \text { for all } u, v \in \Sigma^{*} \text { and } a \in \Sigma .
$$

Similarly, we say $\leqslant$ is a left quasiorder, and denote it $b y \leqslant^{\ell}$, iff

$$
u \leqslant^{\ell} v \Rightarrow a u \leqslant^{\ell} a v, \quad \text { for all } u, v \in \Sigma^{*} \text { and } a \in \Sigma
$$

A qoset $\langle D, \leqslant\rangle$ is a partially ordered set (poset for short) when $\leqslant$ is antisymmetric. A subset $X \subseteq D$ of a poset is directed iff $X$ is nonempty and every pair of elements in $X$ has an upper bound in $X$.

Definition (Least Upper Bound). Let $\langle D, \leqslant\rangle$ be a partially ordered set and let $x, y \in$ $D$. The least upper bound of $x$ and $y$ is the element $z \in D$ such that

$$
x \leqslant z \wedge y \leqslant z \wedge(\forall d \in D,(x \leqslant d \wedge y \leqslant d) \Rightarrow z \leqslant d) .
$$

Definition (Greatest Lower Bound). Let $\langle D, \leqslant\rangle$ be a partially ordered set and let $x, y \in D$. The greatest lower bound of $x$ and $y$ is the element $z \in D$ such that

$$
z \leqslant x \wedge z \leqslant y \wedge(\forall d \in D,(d \leqslant x \wedge d \leqslant y) \Rightarrow d \leqslant z) .
$$

A poset $\langle D, \leqslant\rangle$ is a directed-complete partial order (CPO for short) iff it has the least upper bound (lub for short) of all its directed subsets. A poset is a joinsemilattice iff it has the lub of all its nonempty finite subsets (therefore binary lubs are enough). A poset is a complete lattice iff it has the lub of all its arbitrary (possibly empty) subsets; in this case, let us recall that it also has the greatest lower bound (glb for short) of all its arbitrary subsets. 


\section{Well-quasiorders}

Definition (Antichain). Let $\langle D, \leqslant\rangle$ be a qoset. A subset $X \subseteq D$ is an antichain iff any two distinct elements in $X$ are incomparable.

We denote the set of antichains of a qoset $\langle D, \leqslant\rangle$ by

$$
\mathrm{AC}_{\langle D, \leqslant\rangle} \stackrel{\text { def }}{=}\{X \subseteq D \mid X \text { is an antichain }\} .
$$

Definition (Well-quasiorder). Let $\langle D, \leqslant\rangle$ be a quasiordered set. We say it is a wellquasiordered set (wqoset for short), and $\leqslant$ is a well-quasiorder (wqo for short), iff for every countably infinite sequence of elements $\left\{x_{i}\right\}_{i \in \mathbb{N}}$ there exist $i, j \in \mathbb{N}$ such that $i<j$ and $x_{i} \leqslant x_{j}$.

Equivalently, we say $\langle D, \leqslant\rangle$ is a well-quasiordered set iff $D$ is DCC and D has no infinite antichain.

For every qoset $\langle D, \leqslant\rangle$, we shift the quasiorder $\leqslant$ to a binary relation $\sqsubseteq \leqslant$ on the powerset as follows. Given $X, Y \in \wp(D)$,

$$
X \sqsubseteq \leqslant \stackrel{\text { def }}{\Longleftrightarrow} \forall x \in X, \exists y \in Y, y \leqslant x .
$$

When the quasiorder is clear from the context, we drop the subindex and write simply $\sqsubseteq$. Given a qoset $\langle D, \leqslant\rangle$, we define the set of minimal elements of a subset $X \subseteq D$ :

$$
\min _{\leqslant}(X) \stackrel{\text { def }}{=}\{x \in X \mid \forall y \in X, y \leqslant x \Rightarrow y=x\} .
$$

Definition (Minor). Let $\langle D, \leqslant\rangle$ be a qoset. A minor of a subset $X \subseteq D$, denoted by $\lfloor X\rfloor$, is a subset of the minimal elements of $X$ w.r.t. $\leqslant$, i.e. $\lfloor X\rfloor \subseteq \min _{\leqslant}(X)$, such that $X \sqsubseteq\lfloor X\rfloor$ holds.

Clearly, a minor $\lfloor X\rfloor$ of some set $X$ is always an antichain.

Let us recall that every subset $X$ of a wqoset $\langle D, \leqslant\rangle$ has at least one minor set, all minor sets of $X$ are finite, $\lfloor\{x\}\rfloor=\{x\},\lfloor\varnothing\rfloor=\varnothing$, and if $\langle D, \leqslant\rangle$ is additionally a poset then there exists exactly one minor set of $X$. It turns out that $\left\langle\mathrm{AC}_{\langle D, \leqslant\rangle}, \sqsubseteq\right\rangle$ is a qoset which is ACC if $\langle D, \leqslant\rangle$ is a wqoset and is a poset if $\langle D, \leqslant\rangle$ is a poset.

\section{Nerode Quasiorders}

Definition (Nerode's Quasiorders). Let $L \subseteq \Sigma^{*}$ be a language. The left and right Nerode's quasiorders on $\Sigma^{*}$ are, respectively

$$
u \leqslant_{L}^{\ell} v \stackrel{\text { def }}{\Leftrightarrow} L u^{-1} \subseteq L v^{-1}, \quad u \leqslant_{L}^{r} v \stackrel{\text { def }}{\Leftrightarrow} u^{-1} L \subseteq v^{-1} L
$$


As shown by de Luca and Varricchio [1994], $\leqslant_{L}^{\ell}$ and $\leqslant_{L}^{r}$ are, respectively, left and right monotone and, if $L$ is regular then both $\leqslant_{L}^{\ell}$ and $\leqslant_{L}^{r}$ are wqos [de Luca and Varricchio 1994, Theorem 2.4].

Furthermore, de Luca and Varricchio [1994] showed that $\leqslant_{L}^{\ell}$ is maximum in the set of all left monotone quasiorders $\leqslant^{\ell}$ that satisfy $\rho_{\leqslant \ell}(L)=L$. Therefore, for every left quasiorder $\leqslant^{\ell}$, if $\rho_{\leqslant \ell}(L)=L$ then $x \leqslant^{\ell} y \Rightarrow x \leqslant_{L}^{\ell} y$. Similarly holds for right quasiorders and the right Nerode quasiorder.

\subsection{Kleene Iterates}

Let $\langle X, \leqslant\rangle$ be a qoset and $f: X \rightarrow X$ be a function. The function $f$ is monotone iff $x \leqslant y$ implies $f(x) \leqslant f(y)$. Given $b \in X$, the trace of values of the variable $x \in X$ computed by the following iterative procedure:

$$
\operatorname{KLEEnE}(f, b) \stackrel{\text { def }}{=}\left\{\begin{array}{l}
x:=b ; \\
\text { while } f(x) \neq x \text { do } x:=f(x) ; \\
\text { return } x ;
\end{array}\right.
$$

provides the possibly infinite sequence of so-called Kleene iterates of the function $f$ starting from the basis $b$.

Whenever $\langle X, \leqslant\rangle$ is an ACC (resp. DCC) CPO, $b \leqslant f(b)$ (resp. $f(b) \leqslant b$ ) and $f$ is monotone then, by Knaster-Tarski-Kleene fixpoint theorem, $\operatorname{KLeEne}(f, b)$ terminates and returns the least (resp. greatest) fixpoint of the function $f$ which is greater (resp. lower) than or equal to $b$. In particular, if $\perp_{X}$ (resp. $T_{X}$ ) is the least (resp. greatest) element of $X$ then $\operatorname{Kueene}\left(f, \perp_{X}\right)\left(\operatorname{resp} . \operatorname{KueEne}\left(f, \top_{X}\right)\right)$ computes the sequence of Kleene iterates that finitely converges to the least (resp. greatest) fixpoint of $f$, denoted by $\operatorname{lfp}(f)(\operatorname{resp} \cdot \operatorname{gfp}(f))$.

TheOrem 3.5.1. Let $\langle X, \leqslant\rangle$ be an ACC CPO and let $f: X \rightarrow X$ be a monotone function. Then $\operatorname{KLEENE}\left(f, \perp_{X}\right)$ terminates and returns the least fixpoint of $f$.

Proof. To simplify the notation, we use $\perp$ to denote the least element of $X, \perp_{X}$. Next, we show by induction that $f^{n}(\perp) \leqslant f^{n+1}(\perp)$ for all $n \geq 0$.

- Base case: The relation $\perp \leqslant f(\perp)$ holds since $\perp$ is the least element in $X$.

- Inductive step: Assume $f^{n}(\perp) \leqslant f^{n+1}(\perp)$ for some value $n$. Then, since $f$ is a monotone function, we have that that $f^{n+1}(\perp) \leqslant f^{n+2}(\perp)$.

We conclude that $f^{n}(\perp) \leqslant f^{n+1}(\perp)$ holds for all $n \geq 0$. Since the qoset $\langle X, \leqslant\rangle$ is an ACC, there is no infinite sequence of ascending elements and, as a consequence, $\operatorname{KLEENE}(f, \perp)$ terminates and returns a fixpoint of function $f$.

Next, we show that if $f^{n}(\perp)=f^{n+1}(\perp)$ for some $n$ then $f^{n}(\perp)=\operatorname{lfp}(f)$. 
To do that, we show that $f^{i}(\perp) \leqslant p$ for every $i \geq 0$ and for every fixpoint $p$ of $f$. Therefore, the fixpoint $f^{n}(\perp)$ is below (for the quasiorder $\leqslant$ ) than any other fixpoint, hence $f^{n}(\perp)$ is the least fixpoint of $f$, i.e. $f^{n}(\perp)=\operatorname{lfp}(f)$.

Again, we proceed by induction in $n$. Let $p$ be a fixpoint of $f$, i.e. $f(p)=p$.

- Base case: The relation $\perp \leqslant p$ trivially holds by definition of $\perp$.

- Inductive step: Assume $f^{n}(\perp) \leqslant p$ for some value $n$. Then, since $f$ is a monotone function, we have that that $f^{n+1}(\perp) \leqslant f(p)=p$, where the last equality follows from the fact that $p$ is a fixpoint.

Clearly, $f^{n}(\perp) \leqslant p$ for all $n \geq 0$ and for all fixpoint $p$ of $f$. We conclude that $\operatorname{KLEENE}(f, \perp)=\operatorname{lfp}(f)$.

For the sake of clarity, we overload the notation and use the same symbol for a function/relation and its componentwise (i.e. pointwise) extension on product domains. For instance, if $f: X \rightarrow Y$ then $f$ also denotes the standard product function $f: X^{n} \rightarrow Y^{n}$ defined by $\lambda\left\langle x_{1}, \ldots, x_{n}\right\rangle \in X^{n} .\left\langle f\left(x_{1}\right), \ldots, f\left(x_{n}\right)\right\rangle$. A vector $\vec{Y}$ in some product domain $D^{|S|}$ is also denoted by $\left\langle Y_{i}\right\rangle_{i \in S}$ and, for some $i \in S$, $\vec{Y}_{i}$ denotes its component $Y_{i}$.

\subsection{Closures and Galois Connections}

We conclude this chapter by recalling some basic notions on closure operators and Galois Connections commonly used in abstract interpretation (see, e.g., [Cousot and Cousot 1979; Miné 2017]).

Closure operators and Galois Connections are equivalent notions [Cousot 1978] and, therefore, they are both used for defining the notion of approximation in abstract interpretation, where closure operators allow us to define and reason on abstract domains independently of a specific representation which is required by Galois Connections.

Definition (Upper Closure Operator). Let $\left\langle C, \leqslant_{C}, \vee, \wedge\right\rangle$ be a complete lattice, where $\vee$ and $\wedge$ denote, respectively, the lub and glb. An upper closure operator, or simply closure, on $\left\langle C, \leqslant_{C}\right\rangle$ is a function $\rho: C \rightarrow C$ which is:

(i) monotone, i.e. $x \leqslant_{C} y \Rightarrow \rho(x) \leqslant_{C} \rho(y)$ for all $x, y \in C$;

(ii) idempotent, i.e. $\rho(\rho(x))=\rho(x)$ for all $x \in C$, and

(iii) extensive, i.e. $x \leqslant_{C} \rho(x)$ for all $x \in C$.

The set of all upper closed operators on $C$ is denoted by uco $(C)$. We often write $c \in \rho(C)$, or simply $c \in \rho$, to denote that there exists $c^{\prime} \in C$ such that 
$c=\rho\left(c^{\prime}\right)$, and recall that this happens iff $\rho(c)=c$. If $\rho \in \operatorname{uco}(C)$ then for all $c_{1} \in C, c_{2} \in \rho$ and $X \subseteq C$, it turns out that:

$$
\begin{aligned}
& c_{1} \leqslant_{C} c_{2} \Leftrightarrow \rho\left(c_{1}\right) \leqslant_{C} \rho\left(c_{2}\right) \Leftrightarrow \rho\left(c_{1}\right) \leqslant_{C} c_{2} \\
& \rho(\vee X)=\rho(\vee \rho(X)) \text { and } \wedge \rho(X)=\rho(\wedge \rho(X)) .
\end{aligned}
$$

In abstract interpretation, a closure operator $\rho \in \operatorname{uco}(C)$ on a concrete domain $C$ plays the role of abstraction function for objects of $C$. Given two closures $\rho, \rho^{\prime} \in \operatorname{uco}(C), \rho$ is a coarser abstraction than $\rho^{\prime}$ (or, equivalently, $\rho^{\prime}$ is a more precise abstraction than $\rho$ ) iff the image of $\rho$ is a subset of the image of $\rho^{\prime}$, i.e. $\rho(C) \subseteq \rho^{\prime}(C)$, and this happens iff for any $x \in C, \rho^{\prime}(x) \leqslant_{C} \rho(x)$.

Definition (Galois Connection). A Galois Connection (GC for short) or adjunction between two posets $\left\langle C, \leqslant_{C}\right\rangle$ (a concrete domain) and $\left\langle A, \leqslant_{A}\right\rangle$ (an abstract domain) consists of two monotone functions $\alpha: C \rightarrow A$ and $\gamma: A \rightarrow C$ such that

$$
\alpha(c) \leqslant_{A} a \Leftrightarrow c \leqslant_{C} \gamma(a), \quad \text { for all } a \in A, c \in C .
$$

A Galois Connection is denoted by $\left\langle C, \leqslant_{C}\right\rangle \underset{\alpha}{\stackrel{\gamma}{\leftrightarrows}}\left\langle A, \leqslant_{A}\right\rangle$.

LEмmA 3.6.1. Let $\left\langle C, \leqslant_{C}\right\rangle \underset{\alpha}{\stackrel{\gamma}{\leftrightarrows}}\left\langle A, \leqslant_{A}\right\rangle$ be a GC. The following properties hold:

(a) $x \leqslant C \gamma \circ \alpha(x)$ and $\alpha \circ \gamma(y) \leqslant A y$.

(b) $\alpha$ and $\gamma$ are monotonic functions.

(c) $\alpha=\alpha \circ \gamma \circ \alpha$ and $\gamma=\gamma \circ \alpha \circ \gamma$.

Proof.

(a) Since $\leqslant_{A}$ is reflexive, we have that for all $x \in A \alpha(x) \leqslant_{A} \alpha(x)$ holds and, by definition of GC, $\alpha(x) \leqslant_{A} \alpha(x) \Leftrightarrow x \leqslant_{C} \gamma(\alpha(x))$. Therefore, $x \leqslant_{C} \gamma(\alpha(x))$.

Similarly, since $\alpha(\gamma(y)) \leqslant_{A} y \Leftrightarrow \gamma(y) \leqslant_{C} \gamma(y)$ and $\gamma(y) \leqslant_{C} \gamma(y)$, we conclude that $\alpha(\gamma(y)) \leqslant A y$.

(b) Let $c, c^{\prime} \in C$ be such that $c \leqslant_{C} c^{\prime}$. Then, by Lemma 3.6.1 (a), we have that $c^{\prime} \leqslant_{C} \gamma\left(\alpha\left(c^{\prime}\right)\right)$ and, by definition of GC, $c \leqslant_{C} \gamma\left(\alpha\left(c^{\prime}\right)\right) \Rightarrow \alpha(c) \leqslant_{A} \alpha\left(c^{\prime}\right)$.

Similarly, let $a, a^{\prime} \in A$ be such that $a \leqslant_{A} a^{\prime}$. Then, by Lemma 3.6.1 (a), we have that $\alpha(\gamma(a)) \leqslant_{A} a^{\prime}$, hence $\alpha\left(\gamma(a) \leqslant_{A} a^{\prime} \Rightarrow \gamma(a) \leqslant_{A} \gamma\left(a^{\prime}\right)\right.$.

(c) Let $c \in C$. By Lemma 3.6.1 (a), $c \leqslant_{C} \gamma(\alpha(c))$ which, by Lemma 3.6.1 (b), implies that $\alpha(c) \leqslant_{A} \alpha(\gamma(\alpha(c)))$. Moreover, since $\gamma(\alpha(c)) \leqslant_{C} \gamma(\alpha(c))$, it follows from the definition of GC that $\alpha(\gamma(\alpha(c))) \leqslant_{A} \alpha(c)$. Therefore $\alpha(\gamma(\alpha(c)))=\alpha(c)$.

Similarly, let $a \in A$. By Lemma 3.6.1 (a) and (b), $\gamma(\alpha(\gamma(a))) \leqslant_{A} \gamma(a)$ 
and, since $\alpha(\gamma(a)) \leqslant_{C} \alpha(\gamma(a))$, it follows from the definition of GC that $\gamma(a) \leqslant_{A} \gamma(\alpha(\gamma(a)))$. Therefore $\gamma(a)=\gamma(\alpha(\gamma(a)))$.

The function $\alpha$ is called the left-adjoint of $\gamma$, and, dually, $\gamma$ is called the rightadjoint of $\alpha$. This terminology is justified by the fact that if a function $\alpha: C \rightarrow A$ admits a right-adjoint $\gamma: A \rightarrow C$ then this is unique (and this dually holds for left-adjoints).

It turns out that, in a GC, $\gamma$ is always co-additive, i.e. it preserves arbitrary glb's, while $\alpha$ is always additive, i.e. it preserves arbitrary lub's. Moreover, an additive function $\alpha: C \rightarrow A$ uniquely determines its right-adjoint by

$$
\gamma \stackrel{\text { def }}{=} \lambda a . \bigvee_{C}\{c \in C \mid \alpha(c) \leqslant A a\}
$$

Dually, a co-additive function $\gamma: A \rightarrow C$ uniquely determines its left-adjoint by

$$
\alpha \stackrel{\text { def }}{=} \lambda c . \bigwedge_{A}\left\{a \in A \mid c \leqslant_{C} \gamma(a)\right\}
$$

We conclude this chapter with the following lemma, which is folklore in abstract interpretation yet we provide a proof for the sake of completeness.

LEMMA 3.6.2. Let $\left\langle C, \leqslant_{C}\right\rangle \underset{\alpha}{\stackrel{\gamma}{\leftrightarrows}}\left\langle A, \leqslant_{A}\right\rangle$ be a GC between complete lattices and $f: C \rightarrow C$ be a monotone function. Then, $\gamma(\operatorname{lfp}(\alpha f \gamma))=\operatorname{lfp}(\gamma \alpha f)$.

Proof. Let us first show that $\gamma(\operatorname{lfp}(\alpha f \gamma)) \geqslant_{C} \operatorname{lfp}(\gamma \alpha f)$ :

$$
\begin{aligned}
\gamma(\operatorname{lfp}(\alpha f \gamma)) \leqslant_{C} \gamma(\operatorname{lfp}(\alpha f \gamma)) \Leftrightarrow & {[\text { Since } g(\operatorname{lfp}(g))=\operatorname{lfp}(g)] } \\
\gamma \alpha f(\gamma(\operatorname{lfp}(\alpha f \gamma))) \leqslant_{C} \gamma(\operatorname{lfp}(\alpha f \gamma)) \Rightarrow & {[\text { Since } g(x) \leqslant x \Rightarrow \operatorname{lfp}(g) \leqslant x] } \\
\operatorname{lfp}(\gamma \alpha f) \leqslant_{C} \gamma(\operatorname{lfp}(\alpha f \gamma)) &
\end{aligned}
$$

Then, let us prove that $\gamma(\operatorname{lfp}(\alpha f \gamma)) \leqslant_{C} \operatorname{lfp}(\gamma \alpha f)$ :

$$
\begin{array}{rll}
\operatorname{lfp}(\gamma \alpha f) \leqslant_{C} \operatorname{lfp}(\gamma \alpha f) & \Leftrightarrow & {[\text { Since } g(\operatorname{lfp}(g))=\operatorname{lfp}(g)]} \\
\gamma \alpha f(\operatorname{lfp}(\gamma \alpha f)) \leqslant_{C} \operatorname{lfp}(\gamma \alpha f) & \Rightarrow & {[\text { Since } \alpha \text { is monotone] }} \\
\alpha \gamma \alpha f(\operatorname{lfp}(\gamma \alpha f)) \leqslant_{A} \alpha(\operatorname{lfp}(\gamma \alpha f)) \Leftrightarrow & {[\text { Since } \alpha \gamma \alpha=\alpha \text { in GCs] }} \\
\alpha f(\operatorname{lfp}(\gamma \alpha f)) \leqslant_{A} \alpha(\operatorname{lfp}(\gamma \alpha f)) \Leftrightarrow & {[\text { Since } g(\operatorname{lfp}(g))=\operatorname{lfp}(g)]} \\
\alpha f \gamma(\alpha(\operatorname{lfp}(\gamma \alpha f))) \leqslant_{A} \alpha(\operatorname{lfp}(\gamma \alpha f)) \Rightarrow & {[\text { Since } g(x) \leqslant x \Rightarrow \operatorname{lfp}(g) \leqslant x]} \\
\operatorname{lfp}(\alpha f \gamma) \leqslant_{A} \alpha(\operatorname{lfp}(\gamma \alpha f)) \Rightarrow & {[\text { Since } \gamma \text { is monotone }]}
\end{array}
$$




$$
\begin{gathered}
\gamma(\operatorname{lfp}(\alpha f \gamma)) \leqslant_{C} \gamma \alpha(\operatorname{lfp}(\gamma \alpha f)) \Leftrightarrow \quad[\text { Since } g(\operatorname{lfp}(g))=\operatorname{lfp}(g)] \\
\gamma(\operatorname{lfp}(\alpha f \gamma)) \leqslant_{C} \operatorname{lfp}(\gamma \alpha f)
\end{gathered}
$$

\subsection{Complexity Notation}

In this thesis we analyze the time and space complexity of some algorithms and constructions. To do that, we use the standard small-O, big-O and big-Omega notation to compare functions. Next, we define these notations for the shake of completeness, where, given a real number $k$, we write $|k|$ to denote its absolute value.

Definition (Small-O, Big-O, Big-Omega). Let $f$ and $g$ be two functions on the real numbers. Then

$$
\begin{aligned}
& f(n)=o(g(n)) \stackrel{\text { def }}{\Leftrightarrow} \forall k>0, \exists n_{0}, \forall n>n_{0}, f(n) \leq k \cdot g(n) \stackrel{\text { def }}{\Leftrightarrow} \lim _{n \rightarrow \infty} \frac{f(n)}{g(n)}=0 \\
& f(n)=O(g(n)) \stackrel{\text { def }}{\Leftrightarrow} \exists k>0, \exists n_{0}, \forall n>n_{0}, f(n) \leq k \cdot g(n) \stackrel{\text { def }}{\Leftrightarrow} \limsup _{n \rightarrow \infty} \frac{f(n)}{g(n)}<\infty \\
& f(n)=\Omega(g(n)) \stackrel{\text { def }}{\Leftrightarrow} \exists k>0, \exists n_{0}, \forall n>n_{0}, f(n) \geq k \cdot g(n) \stackrel{\text { def }}{\Leftrightarrow} \liminf _{n \rightarrow \infty} \frac{f(n)}{g(n)}>0
\end{aligned}
$$

Intuitively, $f(n)=o(g(n))$ indicates that $f$ is asymptotically dominated by $g$; $f(n)=O(g(n))$ indicates that $f$ is asymptotically bounded above by $g$ and $f(n)=\Omega(g(n))$ indicates that $f$ is asymptotically bounded below by $g$.

These notations allow us to simplify the complexity analysis by removing all components of low impact in a complexity function. For instance, let the number of operations performed by an algorithm on an input of size $n$ be given by a function $f(n)$ that satisfies

$$
n^{2}+n \cdot \log n+k \leq f(n) \leq n^{3}+n^{2}+\log n+k^{\prime},
$$

where $k$ and $k^{\prime}$ are constants. Since, by definition, $n^{2}=o\left(n^{3}\right), \log (n)=o\left(n^{3}\right)$ and $k^{\prime}=o\left(n^{3}\right)$ we find that the components $n^{2}, \log n$ and $k^{\prime}$ have low impact in the behavior of the upper bound of $f(n)$ for large values of $n$. Similarly, the components $n \cdot \log (n)$ and $k$ have low impact in the lower bound of $f(n)$ for large values of $n$. Therefore, we find that $O(f(n))=O\left(n^{3}+n^{2}+\log n+k^{\prime}\right)=O\left(n^{3}\right)$ and $\Omega(f(n))=\Omega\left(n^{2}+n \cdot \log n+k\right)=\Omega\left(n^{2}\right)$. Intuitively, this means that for large values of the parameter $n$ the function $f(n)$ is below $n^{3}$ and above $n^{2}$. 


\section{DECIDING LANGUAgE INCLUSION}

In this chapter, we present a quasiorder-based framework for deciding language inclusion which is a fundamental and classical problem [Hopcroft and Ullman 1979, Chapter 11] with applications to different areas of computer science.

The basic idea of our approach for solving a language inclusion problem $L_{1} \subseteq L_{2}$ is to leverage Cousot and Cousot's abstract interpretation [Cousot and Cousot 1977; 1979] for checking the inclusion of an over-approximation (i.e. a superset) of $L_{1}$ into $L_{2}$. This idea draws inspiration from the work of Hofmann and Chen [2014], who used abstract interpretation to decide language inclusion between languages of infinite words.

Assuming that $L_{1}$ is specified as least fixpoint of an equation system on $\wp\left(\Sigma^{*}\right)$, an over-approximation of $L_{1}$ is obtained by applying an over-approximating abstraction function for sets of words $\rho: \wp\left(\Sigma^{*}\right) \rightarrow \wp\left(\Sigma^{*}\right)$ at each step of the Kleene iterates converging to the least fixpoint $L_{1}$. This abstraction map $\rho$ is an upper closure operator which is used in standard abstract interpretation for approximating an input language by adding words (possibly none) to it.

This abstract interpretation-based approach provides an abstract inclusion check $\rho\left(L_{1}\right) \subseteq L_{2}$ which is always sound by construction because $L_{1} \subseteq \rho\left(L_{1}\right)$. We then give conditions on $\rho$ which ensure a complete abstract inclusion check, namely, the answer to $\rho\left(L_{1}\right) \subseteq L_{2}$ is always exact (no "false alarms" in abstract interpretation terminology). These conditions are: (i) $\rho\left(L_{2}\right)=L_{2}$ and (ii) $\rho$ is a complete abstraction for symbol concatenation $\lambda X \in \wp\left(\Sigma^{*}\right)$. $a X$, for all $a \in \Sigma$, according to the standard notion of completeness in abstract interpretation [Cousot and Cousot 1977; Giacobazzi et al. 2000; Ranzato 2013]. This approach leads us to design in Section 4.2 two general algorithmic frameworks for language inclusion problems which are parameterized by an underlying language abstraction 
(see Theorems 4.2.10 and 4.2.11). Intuitively, the first of these frameworks allows us to decide the inclusion $L_{1} \subseteq L_{2}$ by manipulating finite sets of words, even if the languages $L_{1}$ and $L_{2}$ are infinite. On the other hand, the second framework allows us to decide the inclusion by working on an abstract domain.

We then focus on over-approximating abstractions $\rho$ which are induced by a quasiorder relation $\leqslant$ on words in $\Sigma^{*}$. Here, a language $L$ is over-approximated by adding all the words which are "greater than or equal to" some word of $L$ for $\leqslant$. This allows us to instantiate the above conditions (i) and (ii) for having a complete abstract inclusion check in terms of the quasiorder $\leqslant$. Termination, which corresponds to having finitely many Kleene iterates in the fixpoint computations, is guaranteed by requiring that the relation $\leqslant$ is a well-quasiorder.

We define quasiorders satisfying the above conditions which are directly derived from the standard Nerode equivalence relations on words. These quasiorders have been first investigated by Ehrenfeucht et al. [1983] and have been later generalized and extended by de Luca and Varricchio [1994; 2011]. In particular, drawing from a result by de Luca and Varricchio [1994], we show that the language abstractions induced by the Nerode's quasiorders are the most general ones (thus, intuitively optimal) which fit in our algorithmic framework for checking language inclusion.

While these quasiorder abstractions do not depend on some language representation (e.g., some class of automata), we provide quasiorders which instead exploit an underlying language representation given by a finite automaton. In particular, by selecting suitable well-quasiorders for the class of language inclusion problems at hand, we are able to systematically derive decision procedures for the inclusion problem $L_{1} \subseteq L_{2}$ when: (i) both $L_{1}$ and $L_{2}$ are regular, (ii) $L_{1}$ is regular and $L_{2}$ is the trace language of a one-counter net and (iii) $L_{1}$ is contextfree and $L_{2}$ is regular.

These decision procedures that we systematically derive here by instantiating our framework are then related to existing language inclusion checking algorithms. We study in detail the case where both languages $L_{1}$ and $L_{2}$ are regular and represented by finite-state automata. When our decision procedure for $L_{1} \subseteq L_{2}$ is derived from a well-quasiorder on $\Sigma^{*}$ by exploiting the automatonbased representation of $L_{2}$, it turns out that we obtain the well-known "antichains algorithm" by Wulf et al. [2006]. Also, by including a simulation relation in the definition of the well-quasiorder we derive a decision procedure that partially matches the language inclusion algorithm by Abdulla et al. [2010], and in turn also that by Bonchi and Pous [2013]. For the case in which $L_{1}$ is regular and $L_{2}$ is the set of traces of a one-counter net we derive an alternative proof for the decidability of the language inclusion problem [Jancar et al. 1999]. Moreover, for the 
case in which $L_{1}$ is context-free and $L_{2}$ is regular, we derive a decision procedure that matches the "antichains algorithm" for context-free languages presented by Holík and Meyer [2015].

Finally, we leverage a standard duality result [Cousot 2000] and put forward a greatest fixpoint approach (instead of the above least fixpoint-based procedures) for the case where both $L_{1}$ and $L_{2}$ are regular languages. In this case, we exploit the properties of the over-approximating abstraction induced by the quasiorder in order to show that the Kleene iterates of this greatest fixpoint computation are finitely many. Interestingly, the Kleene iterates of the greatest fixpoint are finitely many whether you apply the over-approximating abstraction or not, which we show by relying on so-called forward complete abstract interpretations [Giacobazzi and Quintarelli 2001].

\subsection{Inclusion Check by Complete Abstractions}

The language inclusion problem consists in checking whether $L_{1} \subseteq L_{2}$ holds where $L_{1}$ and $L_{2}$ are two languages over a common alphabet $\Sigma$. In this section, we show how complete abstractions $\rho$ of $\wp\left(\Sigma^{*}\right)$ can be used to compute an overapproximation $\rho\left(L_{1}\right)$ of $L_{1}$ such that $\rho\left(L_{1}\right) \subseteq L_{2} \Leftrightarrow L_{1} \subseteq L_{2}$.

Closure-based abstract interpretation can be applied to solve a generic inclusion problem by leveraging backward complete abstractions [Cousot and Cousot 1977; 1979; Giacobazzi et al. 2000; Ranzato 2013]. An upper closure $\rho \in \operatorname{uco}(C)$ is called backward complete for a concrete monotone function $f: C \rightarrow C$ when $\rho f=\rho f \rho$ holds. Since $\rho f(c) \leq_{C} \rho f \rho(c)$ always holds for all $c \in C$, the intuition is that backward completeness models an ideal situation where no loss of precision is accumulated in the computations of $\rho f$ when its concrete input objects $c$ are over-approximated by $\rho(c)$. It is well known [Cousot and Cousot 1979] that backward completeness implies completeness of least fixpoints, namely

$$
\rho f=\rho f \rho \Rightarrow \rho(\operatorname{lfp}(f))=\operatorname{lfp}(\rho f)=\operatorname{lfp}(\rho f \rho)
$$

provided that these least fixpoints exist (this is the case, for instance, when $C$ is a CPO). Theorem 4.1.1 states how a concrete inclusion check lfp $(f) \leq_{C} c_{2}$ can be equivalently performed in a backward complete abstraction $\rho$ when $c_{2} \in \rho$.

THEOREM 4.1.1. If $C$ is a $C P O, f: C \rightarrow C$ is monotone, $\rho \in \operatorname{uco}(C)$ is backward complete for $f$ and $c_{2} \in \rho$, then

$$
\operatorname{lfp}(f) \leq_{C} c_{2} \Leftrightarrow \operatorname{lfp}(\rho f) \leq_{C} c_{2} .
$$

In particular, if $\left\langle C, \leq_{C}\right\rangle$ is ACC then the Kleene iterates of $\operatorname{lfp}(\rho f)$ are finitely many. 
Proof. First, we show that $\operatorname{lfp}(f) \leq_{C} c_{2} \Leftrightarrow \operatorname{lfp}(\rho f) \leq_{C} c_{2}$.

$$
\begin{array}{rll}
\operatorname{lfp}(f) \leq_{C} c_{2} \Leftrightarrow & {\left[\text { Since } c_{2} \in \rho\right]} \\
\operatorname{lfp}(f) \leq_{C} \rho\left(c_{2}\right) \Leftrightarrow & {[\text { Since } x \leq \rho(y) \Leftrightarrow \rho(x) \leq \rho(y)]} \\
\rho(\operatorname{lfp}(f)) \leq_{C} \rho\left(c_{2}\right) \Leftrightarrow & {[\text { By Equation }(4.1)]} \\
\operatorname{lfp}(\rho f) \leq_{C} \rho\left(c_{2}\right) \Leftrightarrow & {\left[\text { Since } c_{2} \in \rho\right]} \\
\operatorname{lfp}(\rho f) \leq_{C} c_{2} & &
\end{array}
$$

It remains to prove that the Kleene iterates of $\operatorname{lfp}(\rho f)$ are finitely many. Observe that, since $\rho$ and $f$ are monotone and $\perp_{C} \rho f(\perp)$, we have that

$$
(\rho f)^{n}(\perp) \leq_{C}(\rho f)^{n+1}(\perp) \text { for all } n \geq 1 .
$$

If $\left\langle C, \leq_{C}\right\rangle$ is ACC then, by definition, there are no infinite ascending chains, hence the sequence of Kleene iterates

$$
\perp \leq_{C} \rho f(\perp) \leq_{C}(\rho f)^{2}(\perp) \leq_{C} \ldots \leq_{C}(\rho f)^{n}(\perp)
$$

converges in finitely many steps.

In the following, we will apply this general abstraction scheme to a number of different language inclusion problems, by designing inclusion algorithms which rely on several different backward complete abstractions of $\wp\left(\Sigma^{*}\right)$.

\subsection{An Algorithmic Framework for Language Inclusion}

\subsubsection{Languages as Fixed Points}

Let $\mathcal{N}=\langle Q, \Sigma, \delta, I, F\rangle$ be an NFA. Recall that the language accepted by $\mathcal{N}$ is given by $\mathcal{L}(\mathcal{N}) \stackrel{\text { def }}{=} W_{I, F}^{\mathcal{N}}$ and, therefore,

$$
\mathcal{L}(\mathcal{N})=\cup_{q \in I} W_{q, F}^{\mathcal{N}}=\bigcup_{q \in F} W_{I, q}^{\mathcal{N}}
$$

where, as usual, $\bigcup \varnothing=\varnothing$.

Let us recall how to define the language accepted by an automaton as a solution of a set of equations [Schützenberger 1963]. To do that, given a generic boolean predicate $p(x)$ (typically a membership predicate) on some set and two generic sets $T$ and $F$, we define the following parametric choice function:

$$
\psi_{F}^{T}(p(x)) \stackrel{\text { def }}{=}\left\{\begin{array}{ll}
T & \text { if } p(x) \text { holds } \\
F & \text { otherwise }
\end{array} .\right.
$$


The NFA $\mathcal{N}$ induces the following set of equations, where the $X_{q}$ 's are variables of type $X_{q} \in \wp\left(\Sigma^{*}\right)$ and are indexed by states $q \in Q$ :

$$
\operatorname{Eqn}(\mathcal{N}) \stackrel{\text { def }}{=}\left\{X_{q}=\psi_{\varnothing}^{\{\epsilon\}}(q \in F) \cup \bigcup_{a \in \Sigma, q^{\prime} \in \delta(q, a)} a X_{q^{\prime}} \mid q \in Q\right\}
$$

Thus, the functions in the right-hand side of the equations in $\operatorname{Eqn}(\mathcal{N})$ have type $\wp\left(\Sigma^{*}\right)^{|Q|} \rightarrow \wp\left(\Sigma^{*}\right)$. Since $\left\langle\wp\left(\Sigma^{*}\right)^{|Q|}, \subseteq\right\rangle$ is a (product) complete lattice (because $\left\langle\wp\left(\Sigma^{*}\right), \subseteq\right\rangle$ is a complete lattice) and all the right-hand side functions in $\operatorname{Eqn}(\mathcal{N})$ are clearly monotone, the least solution $\left\langle Y_{q}\right\rangle_{q \in Q} \in \wp\left(\Sigma^{*}\right)^{|Q|}$ of $\operatorname{Eqn}(\mathcal{N})$ does exist and it is easy to check that for every $q \in Q, Y_{q}=W_{q, F}^{\mathcal{N}}$ holds, hence, by Equation (4.2), $\mathcal{L}(\mathcal{N})=\bigcup_{q_{i} \in I} Y_{q_{i}}$.

It is worth noticing that, by relying on right concatenations rather than left ones $a X_{q^{\prime}}$ used in $\operatorname{Eqn}(\mathcal{N})$, one could also define a set of symmetric equations whose least solution coincides with $\left\langle W_{I, q}^{\mathcal{N}}\right\rangle_{q \in Q}$ instead of $\left\langle W_{q, F}^{\mathcal{N}}\right\rangle_{q \in Q}$.

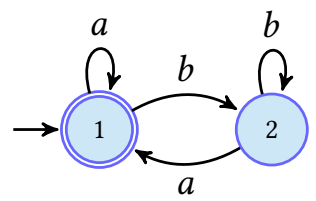

Figure 4.1: An NFA $\mathcal{N}$ with $\mathcal{L}(\mathcal{N})=\left(a+\left(b^{+} a\right)\right)^{*}$.

Example 4.2.1. Let us consider the automaton $\mathcal{N}$ in Figure 4.1. The set of equations induced by $\mathcal{N}$ are as follows:

$$
\operatorname{Eqn}(\mathcal{N})=\left\{\begin{array}{l}
X_{1}=\{\epsilon\} \cup a X_{1} \cup b X_{2} \\
X_{2}=\varnothing \cup a X_{1} \cup b X_{2}
\end{array}\right.
$$

It is convenient to state the equations in $\operatorname{Eqn}(\mathcal{N})$ by exploiting an "initial" vector $\overrightarrow{\boldsymbol{\epsilon}}^{F} \in \wp\left(\Sigma^{*}\right)^{|Q|}$ and a predecessor function $\operatorname{Pre}_{\mathcal{N}}: \wp\left(\Sigma^{*}\right)^{|Q|} \rightarrow \wp\left(\Sigma^{*}\right)^{|Q|} \mathrm{de}-$ fined as follows:

$$
\overrightarrow{\boldsymbol{\epsilon}} F \stackrel{\text { def }}{=}\left\langle\psi_{\varnothing}^{\{\epsilon\}}(q \in F)\right\rangle_{q \in Q}, \quad \operatorname{Pre}_{\mathcal{N}}\left(\left\langle X_{q}\right\rangle_{q \in Q}\right) \stackrel{\text { def }}{=}\left\langle\bigcup_{a \in \Sigma, q^{\prime} \in \delta(q, a)} a X_{q^{\prime}}\right\rangle_{q \in Q} .
$$

The intuition for the function $\operatorname{Pre}_{\mathcal{N}}$ is that given the language $W_{q^{\prime}, F}^{\mathcal{N}}$ and a transition $q^{\prime} \in \delta(q, a)$, we have that $a W_{q^{\prime}, F}^{\mathcal{N}} \subseteq W_{q, F}^{\mathcal{N}}$ holds, i.e. given a subset $X_{q}^{\prime}$ of the language generated by $\mathcal{N}$ from some state $q^{\prime}$, the function Pre $\mathcal{N}_{\mathcal{N}}$ computes a subset $X_{q}$ of the language generated by $\mathcal{N}$ for its predecessor state $q$.

Since $\epsilon \in W_{q, F}^{\mathcal{N}}$ for all $q \in F$, the least fixpoint computation can start from the vector $\overrightarrow{\boldsymbol{\epsilon}}^{F}$ and iteratively apply Pre $_{\mathcal{N}}$. Therefore

$$
\left\langle W_{q, F}^{\mathcal{N}}\right\rangle_{q \in Q}=\operatorname{lfp}\left(\lambda \overrightarrow{\boldsymbol{X}} \cdot \overrightarrow{\boldsymbol{\epsilon}}^{F} \cup \operatorname{Pre}_{\mathcal{N}}(\vec{X})\right)
$$


Together with Equation (4.2), it follows that $\mathcal{L}(\mathcal{N})$ equals the union of the component languages of the vector $\operatorname{lfp}\left(\lambda \vec{X} . \vec{\epsilon}^{F} \cup \operatorname{Pre}_{\mathcal{N}}(\vec{X})\right)$ indexed by the initial states in $I$.

Example 4.2.2 (Continuation of Example 4.2.1). The fixpoint characterization of the vector $\left\langle W_{q, F}^{\mathcal{N}}\right\rangle_{q \in Q}$ is:

$$
\left(\begin{array}{c}
W_{q_{1}, q_{1}}^{\mathcal{N}} \\
W_{q_{2}, q_{1}}^{\mathcal{N}}
\end{array}\right)=\operatorname{lfp}\left(\lambda\left(\begin{array}{c}
X_{1} \\
X_{2}
\end{array}\right) \cdot\left(\begin{array}{c}
\{\epsilon\} \cup a X_{1} \cup b X_{2} \\
\varnothing \cup a X_{1} \cup b X_{2}
\end{array}\right)\right)=\left(\begin{array}{c}
\left(a+\left(b^{+} a\right)\right)^{*} \\
(a+b)^{*} a
\end{array}\right) \cdot \diamond
$$

\section{Fixpoint-based Inclusion Check}

Consider the language inclusion problem $L_{1} \subseteq L_{2}$, where $L_{1}=\mathcal{L}(\mathcal{N})$ for some NFA $\mathcal{N}=\langle Q, \Sigma, \delta, I, F\rangle$. The language $L_{2}$ can be formalized as a vector in $\wp\left(\Sigma^{*}\right)^{|Q|}$ as follows:

$$
\overrightarrow{\mathbf{L}_{2}} \stackrel{\text { def }}{=}\left\langle\psi_{\Sigma^{*}}^{L_{2}}(q \in I)\right\rangle_{q \in Q}
$$

whose components indexed by initial states are $L_{2}$ and those indexed by noninitial states are $\Sigma^{*}$. Then, as a consequence of Equations (4.2), (4.4) and (4.5), we have that

$$
\mathcal{L}(\mathcal{N}) \subseteq L_{2} \Leftrightarrow \operatorname{lfp}\left(\lambda \vec{X} \cdot \overrightarrow{\boldsymbol{\epsilon}}^{F} \cup \operatorname{Pre}_{\mathcal{N}}(\vec{X})\right) \subseteq{\overrightarrow{L_{2}^{I}}}^{I}
$$

\subsubsection{Abstract Inclusion Check using Closures}

In what follows we will apply Theorem 4.1.1 for solving the language inclusion problem where: $C=\left\langle\wp\left(\Sigma^{*}\right)^{|Q|}, \subseteq\right\rangle, f=\lambda \overrightarrow{\boldsymbol{X}} \cdot \overrightarrow{\boldsymbol{\epsilon}}^{F} \cup \operatorname{Pre}_{\mathcal{N}}(\overrightarrow{\boldsymbol{X}})$ and $\rho \in \operatorname{uco}\left(\wp\left(\Sigma^{*}\right)\right)$, so that $\rho \in \operatorname{uco}\left(\wp\left(\Sigma^{*}\right)^{|Q|}\right)$.

THEOREM 4.2.3. Let $\rho \in \operatorname{uco}\left(\wp\left(\Sigma^{*}\right)\right)$ be backward complete for $\lambda X \in \wp\left(\Sigma^{*}\right)$. aX for all $a \in \Sigma$ and let $\mathcal{N}=\langle Q, \Sigma, \delta, I, F\rangle$ be an NFA. Then the extension of $\rho$ to vectors, $\rho \in$ uco $\left(\wp\left(\Sigma^{*}\right)^{|Q|}\right)$, is backward complete for $\operatorname{Pre}_{\mathcal{N}}$ and $\lambda \overrightarrow{\boldsymbol{X}} \cdot \overrightarrow{\boldsymbol{\epsilon}}^{F} \cup \operatorname{Pre}_{\mathcal{N}}(\vec{X})$.

Proof. First, it turns out that:

$$
\begin{aligned}
\rho\left(\operatorname{Pre}_{\mathcal{N}}\left(\left\langle X_{q}\right\rangle_{q \in Q}\right)\right)= & {\left[\text { By definition of Pre } \operatorname{Pr}_{\mathcal{N}}\right] } \\
\rho\left(\bigcup_{a \in \Sigma, q^{\prime} \in \delta(q, a)} a X_{q^{\prime}}\right)= & {[\text { By Equation }(3.2)] } \\
\rho\left(\bigcup_{a \in \Sigma, q^{\prime} \in \delta(q, a)} \rho\left(a X_{q^{\prime}}\right)\right)= & {[\text { By backward completeness of } \rho \text { for } \lambda X . a X] } \\
\rho\left(\bigcup_{a \in \Sigma, q^{\prime} \in \delta(q, a)} \rho\left(a \rho\left(X_{q^{\prime}}\right)\right)\right)= & {[\text { By Equation }(3.2)] } \\
\rho\left(\bigcup_{a \in \Sigma, q^{\prime} \in \delta(q, a)} a \rho\left(X_{q^{\prime}}\right)\right)= & {\left[\text { By definition of Pre } \operatorname{Pr}_{\mathcal{N}}\right] } \\
\rho\left(\operatorname{Pre}_{\mathcal{N}}\left(\rho\left(\left\langle X_{q}\right\rangle_{q \in Q}\right)\right)\right) &
\end{aligned}
$$


Next, we show backward completeness of $\rho$ for $\lambda \vec{X} \cdot \overrightarrow{\boldsymbol{\epsilon}}^{F} \cup \operatorname{Pre}_{\mathcal{N}}(\vec{X})$ :

$$
\begin{aligned}
\rho\left(\overrightarrow{\boldsymbol{\epsilon}}^{F} \cup \operatorname{Pre}_{\mathcal{N}}(\rho(\overrightarrow{\boldsymbol{X}}))\right)= & {[\text { By Equation }(3.2)] } \\
\rho\left(\rho\left(\overrightarrow{\boldsymbol{\epsilon}}^{F}\right) \cup \rho\left(\operatorname{Pre}_{\mathcal{N}}(\rho(\overrightarrow{\boldsymbol{X}}))\right)\right)= & {[\text { By backward completeness of } \rho \text { for Pre }} \\
\rho & \text { ] } \\
\rho\left(\rho\left(\overrightarrow{\boldsymbol{\epsilon}}^{F}\right) \cup \rho\left(\operatorname{Pre}_{\mathcal{N}}(\overrightarrow{\boldsymbol{X}})\right)\right)= & {[\text { By Equation }(3.2)] } \\
\rho\left(\overrightarrow{\boldsymbol{\epsilon}}^{F} \cup \operatorname{Pre}_{\mathcal{N}}(\overrightarrow{\boldsymbol{X}})\right) . &
\end{aligned}
$$

Then, by Equation (4.1), we obtain the following result.

COROLLARY 4.2.4. If $\rho \in \operatorname{uco}\left(\wp\left(\Sigma^{*}\right)\right)$ is backward complete for $\lambda X \in \wp\left(\Sigma^{*}\right)$. aX for all $a \in \sum$ then

$$
\rho\left(\operatorname{lfp}\left(\lambda \overrightarrow{\boldsymbol{X}} \cdot \overrightarrow{\boldsymbol{\epsilon}}^{F} \cup \operatorname{Pre}_{\mathcal{N}}(\overrightarrow{\boldsymbol{X}})\right)\right)=\operatorname{lfp}\left(\lambda \overrightarrow{\boldsymbol{X}} \cdot \rho\left(\overrightarrow{\boldsymbol{\epsilon}}^{F} \cup \operatorname{Pre}_{\mathcal{N}}(\overrightarrow{\boldsymbol{X}})\right)\right)
$$

Note that if $\rho$ is backward complete for $\lambda X . a X$ for all $a \in \sum$ and $L_{2} \in \rho$ then, as a consequence of Theorem 4.1.1 and Corollary 4.2.4, we find that Equivalence (4.6) becomes

$$
\mathcal{L}(\mathcal{N}) \subseteq L_{2} \Leftrightarrow \operatorname{lfp}\left(\lambda \overrightarrow{\boldsymbol{X}} \cdot \rho\left(\overrightarrow{\boldsymbol{\epsilon}}^{F} \cup \operatorname{Pre}_{\mathcal{N}}(\vec{X})\right)\right) \subseteq{\overrightarrow{L_{2}}}^{I}
$$

\subsubsection{Right Concatenation}

Let us consider the symmetric case of right concatenation. Recall that, given an NFA $\mathcal{N}=\langle Q, \Sigma, \delta, I, F\rangle$, we have that

$$
W_{I, q}^{\mathcal{N}}=\psi_{\varnothing}^{\{\epsilon\}}(q \in I) \cup \bigcup_{a \in \Sigma, a \in W_{q^{\prime}, q}^{\mathcal{N}}} W_{I, q^{\prime}}^{\mathcal{N}} a
$$

Correspondingly, we can define a set of fixpoint equations on $\wp\left(\Sigma^{*}\right)$ which is based on right concatenation and is symmetric to Equation (4.3):

$$
\operatorname{Eqn}^{\mathrm{r}}(\mathcal{N}) \stackrel{\text { def }}{=}\left\{X_{q}=\psi_{\varnothing}^{\{\epsilon\}}(q \in I) \cup \bigcup_{a \in \Sigma, q \in \delta\left(q^{\prime}, a\right)} X_{q^{\prime}} a \mid q \in Q\right\}
$$

In this case, if $\vec{Y}=\left\langle Y_{q}\right\rangle_{q \in Q}$ is the least fixpoint solution of $\operatorname{Eqn}^{\mathrm{r}}(\mathcal{N})$ then we have that $Y_{q}=W_{I, q}^{\mathcal{N}}$ for every $q \in Q$. Also, by defining $\overrightarrow{\boldsymbol{\epsilon}}^{I} \in \wp\left(\Sigma^{*}\right)^{|Q|}$ and Post $_{\mathcal{N}}: \wp\left(\Sigma^{*}\right)^{|Q|} \rightarrow \wp\left(\Sigma^{*}\right)^{|Q|}$ as follows:

$$
\overrightarrow{\boldsymbol{\epsilon}} \stackrel{\text { def }}{=}\left\langle\psi_{\varnothing}^{\{\epsilon\}}(q \in I)\right\rangle_{q \in Q}, \quad \operatorname{Post}_{\mathcal{N}}\left(\left\langle X_{q}\right\rangle_{q \in Q}\right) \stackrel{\text { def }}{=}\left\langle\bigcup_{a \in \Sigma, q \in \delta\left(q^{\prime}, a\right)} X_{q^{\prime}} a\right\rangle_{q \in Q},
$$

we have that

$$
\left\langle W_{I, q}\right\rangle_{q \in Q}=\operatorname{lfp}\left(\lambda \vec{X} \cdot \vec{\epsilon}^{I} \cup \operatorname{Post}_{\mathcal{N}}(\vec{X})\right)
$$


Since, by Equation (4.2), we have that $\mathcal{L}(\mathcal{N})=\bigcup_{q \in F} W_{I, q}$, it follows that $\mathcal{L}(\mathcal{N})$ is the union of the component languages of the vector $\operatorname{lfp}\left(\lambda \vec{X} \cdot \vec{\epsilon}^{I} \cup \operatorname{Post}_{\mathcal{N}}(\vec{X})\right)$ indexed by the final states in $F$.

Example 4.2.5. Consider again the NFA $\mathcal{N}$ in Figure 4.1. The set of right equations for $\mathcal{N}$ is:

$$
\operatorname{Eqn}^{\mathrm{r}}(\mathcal{N})=\left\{\begin{array}{l}
X_{1}=\{\epsilon\} \cup X_{1} a \cup X_{2} a \\
X_{2}=\varnothing \cup X_{1} b \cup X_{2} b
\end{array}\right.
$$

so that

$$
\left(\begin{array}{c}
W_{q_{1}, q_{1}} \\
W_{q_{1}, q_{2}}
\end{array}\right)=\operatorname{lfp}\left(\lambda\left(\begin{array}{c}
X_{1} \\
X_{2}
\end{array}\right) \cdot\left(\begin{array}{c}
\{\epsilon\} \cup X_{1} a \cup X_{2} a \\
\varnothing \cup X_{1} b \cup X_{2} b
\end{array}\right)\right)=\left(\begin{array}{c}
\left(a+\left(b^{+} a\right)\right)^{*} \\
a^{*} b\left(b+a^{+} b\right)^{*}
\end{array}\right) \cdot \diamond
$$

Finally, given a language inclusion problem $\mathcal{L}(\mathcal{N}) \subseteq L_{2}$, the language $L_{2}$ can be formalized as the vector

$$
\overrightarrow{\mathbf{L}_{2}} \stackrel{\text { def }}{=}\left\langle\psi_{\Sigma^{*}}^{L_{2}}(q \in F)\right\rangle_{q \in Q} \in \wp\left(\Sigma^{*}\right)^{|Q|},
$$

so that, by Equation (4.8), it turns out that

$$
\mathcal{L}(\mathcal{N}) \subseteq L_{2} \Leftrightarrow \operatorname{lfp}\left(\lambda \vec{X} \cdot \overrightarrow{\boldsymbol{\epsilon}}^{I} \cup \operatorname{Post}_{\mathcal{N}}(\vec{X})\right) \subseteq{\overrightarrow{L_{2}}}^{F}
$$

We therefore have the following symmetric version of Theorem 4.2 .3 for right concatenation.

TheOREM 4.2.6. Let $\rho \in \operatorname{uco}\left(\wp\left(\Sigma^{*}\right)\right)$ be backward complete for $\lambda X \in \wp\left(\Sigma^{*}\right)$.Xa for all $a \in \Sigma$ and let $\mathcal{N}=\langle Q, \Sigma, \delta, I, F\rangle$ be an NFA. Then the extension of $\rho$ to vectors, $\rho \in \operatorname{uco}\left(\wp\left(\Sigma^{*}\right)^{|Q|}\right)$, is backward complete for $\operatorname{Post}_{\mathcal{N}}(\vec{X})$ and $\lambda \vec{X} . \overrightarrow{\boldsymbol{\epsilon}}^{I} \cup \operatorname{Post}_{\mathcal{N}}(\vec{X})$.

Proof. First, it turns out that:

$$
\begin{aligned}
& \left.\rho\left(\operatorname{Post}_{\mathcal{N}}\left(\left\langle X_{q}\right\rangle_{q \in Q}\right)\right)=\quad \text { [By definition of } \text { Post }_{\mathcal{N}}\right] \\
& \rho\left(\bigcup_{a \in \Sigma, q \in \delta\left(q^{\prime}, a\right)} X_{q^{\prime}} a\right)=\quad \text { [By Equation (3.2)] } \\
& \rho\left(\bigcup_{a \in \Sigma, q \in \delta\left(q^{\prime}, a\right)} \rho\left(X_{q^{\prime}} a\right)\right)=\quad \text { By backward completeness of } \rho \text { for } \lambda X . X a \text { ] } \\
& \rho\left(\bigcup_{a \in \Sigma, q \in \delta\left(q^{\prime}, a\right)} \rho\left(\rho\left(X_{q^{\prime}}\right) a\right)\right)=\text { [By Equation (3.2)] } \\
& \left.\rho\left(\bigcup_{a \in \Sigma, q \in \delta\left(q^{\prime}, a\right)} \rho\left(X_{q^{\prime}}\right) a\right)=\quad \text { [By definition of } \text { Post }_{\mathcal{N}}\right] \\
& \rho\left(\operatorname{Post}_{\mathcal{N}}\left(\rho\left(\left\langle X_{q}\right\rangle_{q \in Q}\right)\right)\right) \text {. }
\end{aligned}
$$

Next, we show backward completeness of $\rho$ for $\lambda \vec{X} . \vec{\epsilon}^{I} \cup \operatorname{Post}_{\mathcal{N}}(\vec{X})$ :

$$
\rho\left(\overrightarrow{\boldsymbol{\epsilon}}^{I} \cup \operatorname{Post}_{\mathcal{N}}(\rho(\overrightarrow{\boldsymbol{X}}))\right)=\quad[\text { By Equation (3.2)] }
$$




$$
\begin{aligned}
& \left.\rho\left(\rho\left(\overrightarrow{\boldsymbol{\epsilon}}^{I}\right) \cup \rho\left(\operatorname{Post}_{\mathcal{N}}(\rho(\overrightarrow{\boldsymbol{X}}))\right)\right)=\quad \text { [By backward completeness of } \rho \text { for } \text { Post }_{\mathcal{N}}\right] \\
& \left.\rho\left(\rho\left(\overrightarrow{\boldsymbol{\epsilon}}^{I}\right) \cup \rho\left(\operatorname{Post}_{\mathcal{N}}(\vec{X})\right)\right)=\quad \text { [By Equation }(3.2)\right] \\
& \rho\left(\overrightarrow{\boldsymbol{\epsilon}}^{I} \cup \operatorname{Post}_{\mathcal{N}}(\vec{X})\right) \text {. }
\end{aligned}
$$

\subsubsection{Solving the Abstract Inclusion Check}

In this section we present two techniques for solving the language inclusion problem $\mathcal{L}(\mathcal{N}) \subseteq L_{2}$ by relying on Equivalence (4.7).

The first of these techniques leads to algorithms for solving the inclusion problem by using finite languages. Intuitively, given a closure $\rho$, we show that it is possible to work on the domain $\left\langle\left\{\rho(S) \mid S \in \wp\left(\Sigma^{*}\right)\right\}, \subseteq\right\rangle$ while considering only languages $S$ that are finite.

On the other hand, we present a second technique that relies on the use of Galois Connections in order to solve the language inclusion problem in a different domain. This technique allows us to decide the inclusion $\mathcal{L}(\mathcal{N}) \subseteq L_{2}$ by manipulating the underlying automata representation of the language $L_{2}$.

\subsubsection{Using Finite Languages}

The following result shows that the successive steps of the fixpoint iteration for $\operatorname{lfp}\left(\rho\left(\overrightarrow{\boldsymbol{\epsilon}}^{F} \cup \operatorname{Pre}_{\mathcal{N}}(\overrightarrow{\boldsymbol{X}})\right)\right)$ can be replicated by iterating on a function $f$, instead of $\rho\left(\overrightarrow{\boldsymbol{\epsilon}}^{F} \cup \operatorname{Pre}_{\mathcal{N}}(\overrightarrow{\boldsymbol{X}})\right)$, and then abstracting the result, provided that $f$ meets a set of requirements.

LEMMA 4.2.7. Let $\mathcal{N}=\langle Q, \Sigma, \delta, I, F\rangle$ be an NFA, let $\rho \in \operatorname{uco}\left(\Sigma^{*}\right)$ be backward complete for $\lambda X \in \wp\left(\Sigma^{*}\right)$. aX for all $a \in \Sigma$ and let $f: \wp\left(\Sigma^{*}\right)^{|Q|} \rightarrow \wp\left(\Sigma^{*}\right)^{|Q|}$ be a function such that $\rho\left(\overrightarrow{\boldsymbol{\epsilon}}^{F} \cup \operatorname{Pre}_{\mathcal{N}}(\vec{X})\right)=\rho(f(\vec{X}))$. Then, for all $0 \leq n$,

$$
\left(\rho\left(\overrightarrow{\boldsymbol{\epsilon}}^{F} \cup \operatorname{Pre}_{\mathcal{N}}(\overrightarrow{\boldsymbol{X}})\right)^{n}=\rho\left(f^{n}(\overrightarrow{\boldsymbol{X}})\right) .\right.
$$

Proof. We proceed by induction in $n$.

- Base case: Let $n=0$. Then $f^{0}(\overrightarrow{\boldsymbol{X}})=\left(\rho\left(\overrightarrow{\boldsymbol{\epsilon}}^{F} \cup \operatorname{Pre}_{\mathcal{N}}(\overrightarrow{\boldsymbol{X}})\right)^{0}=\overrightarrow{\boldsymbol{\varnothing}}\right.$.

- Inductive step: Assume that $\rho\left(f^{n}(\overrightarrow{\boldsymbol{X}})\right)=\left(\rho\left(\overrightarrow{\boldsymbol{\epsilon}}^{F} \cup \operatorname{Pre}_{\mathcal{N}}(\overrightarrow{\boldsymbol{X}})\right)^{n}\right.$ holds for some value $n \geq 0$. To simplify the notation, let $\mathcal{P}(\vec{X})=\overrightarrow{\boldsymbol{\epsilon}}^{F} \cup \operatorname{Pre}_{\mathcal{N}}(\vec{X})$ so that $\rho f^{n}=(\rho \mathcal{P})^{n}$. Then

$$
\begin{aligned}
\rho f^{n+1}(\vec{X})= & {\left[\text { Since } f^{n+1}=f^{n} f\right] } \\
\rho f^{n} f(\vec{X})= & {[\text { By Inductive Hypothesis] }}
\end{aligned}
$$




$$
\begin{aligned}
(\rho \mathcal{P})^{n} f(\vec{X})= & {[\text { By Theorem 4.2.3, } \rho \text { is bw. complete for } \mathcal{P}] } \\
(\rho \mathcal{P})^{n} \rho f(\vec{X})= & {[\text { Since } \rho f=\rho \mathcal{P}] } \\
(\rho \mathcal{P})^{n} \rho \mathcal{P}(\vec{X})= & {\left[\text { Since }(\rho \mathcal{P})^{n+1}=(\rho \mathcal{P})^{n} \rho \mathcal{P}\right] } \\
(\rho \mathcal{P})^{n+1}(\vec{X}) &
\end{aligned}
$$

We conclude that $\left(\rho\left(\overrightarrow{\boldsymbol{\epsilon}}^{F} \cup \operatorname{Pre}_{\mathcal{N}}(\overrightarrow{\boldsymbol{X}})\right)^{n}=\rho\left(f^{n}(\overrightarrow{\boldsymbol{X}})\right)\right.$ for all $0 \leq n$.

Lemma 4.2.7 shows that the iterates of $\operatorname{lfp}\left(\rho\left(\overrightarrow{\boldsymbol{\epsilon}}^{F} \cup \operatorname{Pre}_{\mathcal{N}}(\overrightarrow{\boldsymbol{X}})\right)\right)$ can be computed by abstracting the iterates of a function $f$, which might manipulate only finite languages. Moreover, its straightforward to check that Lemma 4.2.7 remains valid when considering a different function $f$ at each step of the iteration as long as all the considered functions satisfy the requirements.

To simplify the notation, given a set of functions $\mathcal{F}$ and a function $f$, we write $\mathcal{F} f$ to denote the composition of one arbitrary function from $\mathcal{F}$ with $f$. Similarly, $f \mathcal{F}$ denotes the composition of $f$ with an arbitrary function from $\mathcal{F}$. Finally, we write $\mathcal{F}^{2}=f$, for instance, to indicate that any composition of two functions in $\mathcal{F}$ equals $f$.

Corollary 4.2.8. Let $\mathcal{N}=\langle Q, \Sigma, \delta, I, F\rangle$ be an NFA, let $\rho \in \operatorname{uco}\left(\Sigma^{*}\right)$ be backward complete for $\lambda X \in \wp\left(\Sigma^{*}\right)$.aX for all $a \in \Sigma$ and let $\mathcal{F}$ be a set of functions such that every function $f \in \mathcal{F}$ is of the form $f: \wp\left(\Sigma^{*}\right)^{|Q|} \rightarrow \wp\left(\Sigma^{*}\right)^{|Q|}$ and satisfies $\rho\left(\overrightarrow{\boldsymbol{\epsilon}}^{F} \cup \operatorname{Pre}_{\mathcal{N}}(\overrightarrow{\boldsymbol{X}})\right)=\rho(f(\overrightarrow{\boldsymbol{X}}))$. Then, for all $0 \leq n$,

$$
\left(\rho\left(\overrightarrow{\boldsymbol{\epsilon}}^{F} \cup \operatorname{Pre}_{\mathcal{N}}(\vec{X})\right)^{n}=\rho\left(\mathcal{F}^{n}(\vec{X})\right) .\right.
$$

Observe that, in particular, Corollary 4.2.8 holds when considering the set $\mathcal{F}=\{f\}$ with $f=\overrightarrow{\boldsymbol{\epsilon}}^{F} \cup \operatorname{Pre}_{\mathcal{N}}(\vec{X})$. Intuitively, this means that we can compute the least fixpoint for $\rho\left(\overrightarrow{\boldsymbol{\epsilon}}^{F} \cup \operatorname{Pre}_{\mathcal{N}}(\overrightarrow{\boldsymbol{X}})\right)$ by iterating on $\overrightarrow{\boldsymbol{\epsilon}}^{F} \cup \operatorname{Pre}_{\mathcal{N}}(\overrightarrow{\boldsymbol{X}})$ until we reach an abstract fixpoint, i.e. the abstraction of two consecutive steps coincide.

The idea of recursively applying a function $f$ until its abstraction reaches a fixpoint is captured by the following definition of the abstract Kleene procedure:

$$
\widehat{\operatorname{KLEENE}}(\operatorname{AbsEq}, f, b) \stackrel{\text { def }}{=}\left\{\begin{array}{l}
x:=b ; \\
\text { while } \neg \operatorname{AbsEq}(f(x), x) \text { do } x:=f(x) ;, \\
\text { return } x ;
\end{array},\right.
$$

where $\operatorname{AbsEq}(x, y)$ is a function that returns true iff the abstraction of $x$ and $y$ coincide, i.e. $\rho(x)=\rho(y)$. Clearly, $\overline{\operatorname{KLEENE}}(i d, f, b)=\operatorname{KLeEne}(f, b)$ where $i d(x, y)$ returns true iff $x=y$. For simplicity, we abuse of notation and write 
$\overline{\operatorname{KLEENE}}(\mathrm{AbsEq}, \mathcal{F}, b)$ to denote the abstract $\overline{\text { KLEENE }}$ iteration where, at each step, an arbitrary function from the set $\mathcal{F}$ is applied.

As the following lemma shows, whenever the domain $\left\langle\left\{\rho(S) \mid S \in \wp\left(\Sigma^{*}\right)\right\}, \subseteq\right\rangle$ is ACC and the abstraction $\rho$ is backward complete for all the functions in the set $\mathcal{F}$, i.e. $\rho \mathcal{F}=\rho \mathcal{F} \rho$, the procedure $\overline{\operatorname{KLEENE}}(\mathrm{AbsEq}, \mathcal{F}, b)$ can be used to compute $\operatorname{lfp}\left(\rho\left(\overrightarrow{\boldsymbol{\epsilon}}^{F} \cup \operatorname{Pre}_{\mathcal{N}}(\overrightarrow{\boldsymbol{X}})\right)\right)$.

LemMA 4.2.9. Let $\mathcal{N}=\langle Q, \Sigma, \delta, I, F\rangle$ be an NFA, let $\rho \in \operatorname{uco}\left(\Sigma^{*}\right)$ be backward complete for $\lambda X \in \wp\left(\Sigma^{*}\right)$. aX for all a $\in \sum$ such that $\left\langle\left\{\rho(S) \mid S \in \wp\left(\Sigma^{*}\right)\right\}, \subseteq\right\rangle$ is an ACC CPO. Let $\mathcal{F}$ be a set of monotone functions such that every $f \in \mathcal{F}$ is of the form $f: \wp\left(\Sigma^{*}\right)^{|Q|} \rightarrow \wp\left(\Sigma^{*}\right)^{|Q|}$ and satisfies $\rho\left(\overrightarrow{\boldsymbol{\epsilon}}^{F} \cup \operatorname{Pre}_{\mathcal{N}}(\vec{X})\right)=\rho(f(\vec{X}))$. Then,

$$
\operatorname{lfp}\left(\rho\left(\overrightarrow{\boldsymbol{\epsilon}}^{F} \cup \operatorname{Pre}_{\mathcal{N}}(\overrightarrow{\boldsymbol{X}})\right)\right)=\rho(\overline{\operatorname{KLEENE}}(\operatorname{AbsEq}, \mathcal{F}, \overrightarrow{\boldsymbol{\varnothing}})) .
$$

Moreover, the iterates of $\operatorname{KLEENE}\left(\rho\left(\overrightarrow{\boldsymbol{\epsilon}}^{F} \cup \operatorname{Pre}_{\mathcal{N}}(\vec{X})\right), \vec{\varnothing}\right)$ coincide in lockstep with the abstraction of the iterates of $\overline{\operatorname{KLEENE}}(\mathrm{AbsEq}, \mathcal{F}, \vec{\varnothing})$.

Proof. Since $\left\langle\left\{\rho(S) \mid S \in \wp\left(\Sigma^{*}\right)\right\}, \subseteq\right\rangle$ is an ACC CPO, by Theorem 3.5.1, we have that

$$
\operatorname{lfp}\left(\rho\left(\overrightarrow{\boldsymbol{\epsilon}}^{F} \cup \operatorname{Pre}_{\mathcal{N}}(\overrightarrow{\boldsymbol{X}})\right)\right)=\operatorname{KLEENE}\left(\rho\left(\overrightarrow{\boldsymbol{\epsilon}}^{F} \cup \operatorname{Pre}_{\mathcal{N}}(\overrightarrow{\boldsymbol{X}})\right), \vec{\varnothing}\right)
$$

On the other hand, by Corollary 4.2.8, the iterates of the above Kleene iteration coincide in lockstep with the abstraction of the iterates of $\overline{\operatorname{KLEENE}}(\mathrm{AbsEq}, \mathcal{F}, \vec{\varnothing})$ and, therefore,

$$
\operatorname{KLEENe}\left(\rho\left(\overrightarrow{\boldsymbol{\epsilon}}^{F} \cup \operatorname{Pre}_{\mathcal{N}}(\vec{X})\right)=\rho(\widehat{\operatorname{KLEENE}}(\operatorname{AbsEq}, \mathcal{F}, \vec{\varnothing}))\right.
$$

As a consequence,

$$
\operatorname{lfp}\left(\rho\left(\overrightarrow{\boldsymbol{\epsilon}}^{F} \cup \operatorname{Pre}_{\mathcal{N}}(\overrightarrow{\boldsymbol{X}})\right)\right)=\rho(\widehat{\operatorname{KLEENE}}(\operatorname{AbsEq}, \mathcal{F}, \vec{\varnothing})) .
$$

The following result relies on the $\overline{\text { KLEENE }}$ procedure to design an algorithm that solves the language inclusion problem $\mathcal{L}(\mathcal{N}) \subseteq L_{2}$ whenever the abstraction $\rho$ and the set of functions $\mathcal{F}$ satisfy a list of requirements in terms of backward completeness and computability.

TheOrem 4.2.10. Let $\mathcal{N}=\langle Q, \Sigma, \delta, I, F\rangle$ be an NFA, let $L_{2}$ be a regular language, let $\rho \in \operatorname{uco}\left(\Sigma^{*}\right)$ and let $\mathcal{F}$ be a set of functions. Assume that the following properties hold:

(i) The abstraction $\rho$ is backward complete for $\lambda X \in \wp\left(\Sigma^{*}\right)$. aX for all $a \in \Sigma$ and satisfies $\rho\left(L_{2}\right)=L_{2}$. 
(ii) The set $\left\langle\left\{\rho(S) \mid S \in \wp\left(\Sigma^{*}\right)\right\}, \subseteq\right\rangle$ is an ACC CPO.

(iii) Every function $f$ in the set $\mathcal{F}$ is of the form $f: \wp\left(\Sigma^{*}\right)^{|Q|} \rightarrow \wp\left(\Sigma^{*}\right)^{|Q|}$, it is computable and satisfies $\rho\left(\overrightarrow{\boldsymbol{\epsilon}}^{F} \cup \operatorname{Pre}_{\mathcal{N}}(\overrightarrow{\boldsymbol{X}})\right)=\rho(f(\overrightarrow{\boldsymbol{X}}))$.

(iv) There is an algorithm, say $\operatorname{AbsEq}^{\sharp}(\vec{X}, \vec{Y})$, which decides the abstraction equivalence $\rho(\vec{X})=\rho(\vec{Y})$, for all $\vec{X}, \vec{Y} \in \wp\left(\Sigma^{*}\right)^{|Q|}$.

(v) There is an algorithm, say $\operatorname{Incl}^{\sharp}(\vec{X})$, which decides the inclusion $\rho(\vec{X}) \subseteq \overrightarrow{\mathbf{L}_{2} I}$, for all $\vec{X} \in \wp\left(\Sigma^{*}\right)^{|Q|}$.

Then, the following is an algorithm which decides whether $\mathcal{L}(\mathcal{N}) \subseteq L_{2}$ :

$\left\langle Y_{q}\right\rangle_{q \in Q}:=\overline{\operatorname{KLEENE}}\left(\mathrm{AbsEq}^{\sharp}, \mathcal{F}, \vec{\varnothing}\right)$;

return Incl ${ }^{\sharp}\left(\left\langle Y_{q}\right\rangle_{q \in Q}\right)$;

Proof. It follows from hypotheses (i), (ii) and (iii), by Lemma 4.2.9, that

$$
\operatorname{lfp}\left(\rho\left(\overrightarrow{\boldsymbol{\epsilon}}^{F} \cup \operatorname{Pre}_{\mathcal{N}}(\overrightarrow{\boldsymbol{X}})\right)\right)=\rho(\overline{\operatorname{KLEENE}}(\operatorname{AbsEq}, \mathcal{F}, \vec{\varnothing}))
$$

The function AbsEq can be replaced by function $\mathrm{AbsEq}^{\sharp}$ due to hypothesis (iv). Moreover, by Equivalence (4.7), which holds by hypothesis (i), and Equation (4.9) we have that

$$
\mathcal{L}(\mathcal{N}) \subseteq L_{2} \Leftrightarrow \rho\left(\widehat{\operatorname{KLEENE}}\left(\operatorname{AbsEq}^{\sharp}, \mathcal{F}, \vec{\varnothing}\right)\right) \subseteq \overrightarrow{\boldsymbol{L}_{2}^{I}} .
$$

Finally, hypotheses (iv) and (v) guarantee, respectively, the decidability of the inclusion $\rho \mathcal{F}(X) \subseteq \rho(X)$ performed at each step of the KLEENE iteration and the decidability of the inclusion of the abstraction of the lfp in $\overrightarrow{\boldsymbol{L}_{2} I}$.

Note that Theorem 4.2.10 can also be stated in a symmetric version for right concatenation similarly to Theorem 4.2.6.

\subsubsection{Using Galois Connections}

The next result reformulates Equivalence (4.7) by using Galois Connections rather than closures, and shows how to design an algorithm that solves a language inclusion problem $\mathcal{L}(\mathcal{N}) \subseteq L_{2}$ on an abstraction $D$ of the concrete domain $\left\langle\wp\left(\Sigma^{*}\right), \subseteq\right\rangle$ whenever $D$ satisfies a list of requirements related to backward completeness and computability.

THEOREM 4.2.11. Let $\mathcal{N}=\langle Q, \Sigma, \delta, I, F\rangle$ be an NFA and $L_{2}$ be a language over $\Sigma$. Let $\left\langle\wp\left(\Sigma^{*}\right), \subseteq\right\rangle \underset{\alpha}{\stackrel{\gamma}{\leftrightarrows}}\left\langle D, \leq_{D}\right\rangle$ be a GC where $\left\langle D, \leq_{D}\right\rangle$ is a poset. Assume that the following properties hold: 
(i) $L_{2} \in \gamma(D)$ and for every $a \in \sum$ and $X \in \wp\left(\Sigma^{*}\right), \gamma \alpha(a X)=\gamma \alpha(a \gamma \alpha(X))$.

(ii) $\left(D, \leq_{D}, \sqcup, \perp_{D}\right)$ is an effective domain, meaning that: $\left(D, \leq_{D}, \sqcup, \perp_{D}\right)$ is an ACC join-semilattice with bottom $\perp_{D}$, every element of $D$ has a finite representation, the binary relation $\leq_{D}$ is decidable and the binary lub $\sqcup$ is computable.

(iii) There is an algorithm, say $\operatorname{Pre}^{\sharp}\left(\vec{X}^{\sharp}\right)$, which computes $\alpha\left(\operatorname{Pre} \mathcal{N}\left(\gamma\left(\vec{X}^{\sharp}\right)\right)\right)$, for all $\vec{X}^{\sharp} \in \alpha\left(\wp\left(\Sigma^{*}\right)\right)|Q|$.

(iv) There is an algorithm, say $\epsilon^{\#}$, which computes $\alpha\left(\overrightarrow{\boldsymbol{\epsilon}}^{F}\right)$.

(v) There is an algorithm, say $\operatorname{Incl} \mathbb{l}^{\sharp}\left(\vec{X}^{\sharp}\right)$, which decides the abstract inclusion $\vec{X}^{\sharp} \leq_{D} \alpha\left(\overrightarrow{L_{2}^{I}}\right)$, for all $\vec{X}^{\sharp} \in \alpha\left(\wp\left(\Sigma^{*}\right)\right)^{|Q|}$.

Then, the following is an algorithm which decides whether $\mathcal{L}(\mathcal{N}) \subseteq L_{2}$ :

$$
\left\langle Y_{q}^{\sharp}\right\rangle_{q \in Q}:=\operatorname{KLEENE}\left(\lambda \vec{X}^{\sharp}, \epsilon^{\sharp} \sqcup \operatorname{Pre}^{\sharp}\left(\vec{X}^{\sharp}\right), \overrightarrow{\perp_{D}}\right) ;
$$

return $\operatorname{Incl}^{\sharp}\left(\left\langle Y_{q}^{\sharp}\right\rangle_{q \in Q}\right)$;

Proof. Let $\rho \stackrel{\text { def }}{=} \gamma \alpha \in \operatorname{uco}\left(\wp\left(\Sigma^{*}\right)\right)$, so that hypothesis (i) can be stated as $L_{2} \in \rho$ and $\rho(a X)=\rho(a \rho(X))$. It turns out that:

$$
\begin{array}{rlrl}
\mathcal{L}(\mathcal{N}) \subseteq L_{2} & \Leftrightarrow & \text { [By Equivalence (4.7)] } \\
\operatorname{lfp}\left(\lambda \overrightarrow{\boldsymbol{X}} \cdot \rho\left(\overrightarrow{\boldsymbol{\epsilon}}^{F} \cup \operatorname{Pre}_{\mathcal{N}}(\overrightarrow{\boldsymbol{X}})\right)\right) \subseteq{\overrightarrow{\boldsymbol{L}_{2}}}^{I} \Leftrightarrow & \text { [By Lemma 3.6.2] } \\
\gamma\left(\operatorname{lfp}\left(\lambda \overrightarrow{\boldsymbol{X}}^{\sharp} \cdot \alpha\left(\overrightarrow{\boldsymbol{\epsilon}}^{F} \cup \operatorname{Pre}_{\mathcal{N}}\left(\gamma\left(\overrightarrow{\boldsymbol{X}}^{\sharp}\right)\right)\right)\right) \subseteq{\overrightarrow{\boldsymbol{L}_{2}^{I}}}^{I} \Leftrightarrow\right. & \text { [By GC] } \\
\gamma\left(\operatorname{lfp}\left(\lambda \overrightarrow{\boldsymbol{X}}^{\sharp} \cdot \alpha\left(\overrightarrow{\boldsymbol{\epsilon}}^{F}\right) \sqcup \alpha\left(\operatorname{Pre}_{\mathcal{N}}\left(\gamma\left(\overrightarrow{\boldsymbol{X}}^{\sharp}\right)\right)\right)\right) \subseteq{\overrightarrow{\boldsymbol{L}_{2}^{I}}}^{I} \Leftrightarrow\right. & \text { [By GC since } \left.L_{2} \in \gamma(D)\right] \\
\operatorname{lfp}\left(\lambda \overrightarrow{\boldsymbol{X}}^{\sharp} \cdot \alpha\left(\overrightarrow{\boldsymbol{\epsilon}}^{F}\right) \sqcup \alpha\left(\operatorname{Pre}_{\mathcal{N}}\left(\gamma\left(\overrightarrow{\boldsymbol{X}}^{\sharp}\right)\right)\right)\right) \leq_{D} \alpha\left({\overrightarrow{\boldsymbol{L}_{2}^{I}}}^{I}\right) & &
\end{array}
$$

By hypotheses (ii), (iii) and (iv), $\operatorname{KLEENE}\left(\lambda \vec{X}^{\sharp} \cdot \epsilon^{\sharp} \sqcup \operatorname{Pre}^{\sharp}\left(\vec{X}^{\sharp}\right), \overrightarrow{\perp_{D}}\right)$ is an algorithm computing the least fixpoint $\operatorname{lfp}\left(\lambda \overrightarrow{\boldsymbol{X}}^{\sharp} \cdot \alpha\left(\overrightarrow{\boldsymbol{\epsilon}}^{F}\right) \sqcup \alpha\left(\operatorname{Pre}_{\mathcal{N}}\left(\gamma\left(\overrightarrow{\boldsymbol{X}}^{\sharp}\right)\right)\right)\right)$. In particular, the hypotheses (ii), (iii) and (iv) ensure that the Kleene iterates of $\lambda \overrightarrow{X^{\sharp}} . \epsilon^{\sharp} \sqcup \operatorname{Pre}{ }^{\sharp}\left(\vec{X}^{\sharp}\right)$ starting from $\overrightarrow{\perp_{D}}$ are in $\alpha\left(\wp\left(\Sigma^{*}\right)\right)^{|Q|}$, computable and finitely many and that it is decidable whether the iterates have reached the fixpoint.

Finally, hypothesis (v) ensures the decidability of the $\leq_{D}$-inclusion check of this least fixpoint in $\alpha\left(\overrightarrow{L_{2}}\right)$.

It is also worth noticing that, analogously to what has been done in Theorem 4.2.6, the above Theorem 4.2 .11 can be also stated in a symmetric version for right (rather than left) concatenation. 


\subsection{Instantiating the Framework}

We instantiate the general algorithmic framework of Section 4.2 to the class of closure operators induced by quasiorder relations on words.

\subsubsection{Word-based Abstractions}

Let $\leqslant \subseteq \Sigma^{*} \times \Sigma^{*}$ be a quasiorder relation on words in $\Sigma^{*}$. Recall that the corresponding closure operator $\rho_{\leqslant} \in \operatorname{uco}\left(\wp\left(\Sigma^{*}\right)\right)$ is defined as follows:

$$
\rho_{\leqslant}(X) \stackrel{\text { def }}{=}\left\{v \in \Sigma^{*} \mid \exists u \in X, u \leqslant v\right\} .
$$

Thus, $\rho_{\leqslant}(X)$ is the $\leqslant$-upward closure of $X$ and it is easy to check that $\rho_{\leqslant}$is indeed a closure on the complete lattice $\left\langle\wp\left(\Sigma^{*}\right), \subseteq\right\rangle$.

As described in Chapter 3, the quasiorder $\leqslant$ is left-monotone (resp. rightmonotone) iff

$$
\forall x_{1}, x_{2} \in \Sigma^{*}, \forall a \in \Sigma, x_{1} \leqslant x_{2} \Rightarrow a x_{1} \leqslant a x_{2} \quad\left(\text { resp. } x_{1} a \leqslant x_{2} a\right)
$$

In fact, if $x_{1} \leqslant x_{2}$ then Equation (4.11) implies that for all $y \in \Sigma^{*}, y x_{1} \leqslant y x_{2}$ since, by induction on the length $|y| \in \mathbb{N}$, we have that:

(i) if $y=\epsilon$ then $y x_{1} \leqslant y x_{2}$;

(ii) if $y=a v$ with $a \in \Sigma, v \in \Sigma^{*}$ then, by inductive hypothesis, $v x_{1} \leqslant v x_{2}$, so that by (4.11), $y x_{1}=a v x_{1} \leqslant a v x_{2}=y x_{2}$

Definition 4.3.1 ( $L$-Consistent Quasiorder). Let $L \in \wp\left(\Sigma^{*}\right)$. A quasiorder $\leqslant_{L}$ on $\Sigma^{*}$ is called left (resp. right) L-consistent iff

(a) $\leqslant_{L} \cap(L \times \neg L)=\varnothing$;

(b) $\leqslant_{L}$ is left-monotone (resp. right-monotone).

Also, $\leqslant_{L}$ is called $L$-consistent when it is both left and right $L$-consistent.

As the following lemma shows, it turns out that a quasiorder is $L$-consistent iff it induces a closure which includes $L$ in its image and it is backward complete for concatenation.

LеммA 4.3.2. Let $L \in \wp\left(\Sigma^{*}\right)$ and $\leqslant_{L}$ be a quasiorder on $\Sigma^{*}$. Then, $\leqslant_{L}$ is a left (resp. right) $L$-consistent quasiorder on $\Sigma^{*}$ if and only if

(a) $\rho_{\leqslant_{L}}(L)=L$, and

(b) $\rho_{\leqslant_{L}}$ is backward complete for $\lambda X . a X$ (resp. $\lambda X$.Xa) for all $a \in \Sigma$. 
Proof. We consider the left case, the right case is symmetric.

(a) The inclusion $L \subseteq \rho_{\leqslant_{L}}(L)$ always holds because $\rho_{\leqslant_{L}}$ is an upper closure. For the reverse inclusion we have that

$$
\begin{array}{r}
\left.\rho_{\leqslant_{L}}(L) \subseteq L \Leftrightarrow \quad \text { [By definition of } \rho_{\leqslant_{L}}(L)\right] \\
\forall v \in \Sigma^{*},\left(\exists u \in L, u \leqslant_{L} v\right) \Rightarrow v \in L \Leftrightarrow \\
\leqslant_{L} \cap(L \times \neg L)=\varnothing .
\end{array}
$$

Thus, $\rho_{\leqslant L}(L)=L$ iff condition (a) of Definition 4.3.1 holds.

(b) We first prove that if $\leqslant_{L}$ is left-monotone then for all $X \in \wp\left(\Sigma^{*}\right)$ we have that $\rho_{\leqslant_{L}}(a X)=\rho_{\leqslant_{L}}\left(a \rho_{\leqslant_{L}}(X)\right)$ for all $a \in \Sigma$.

Monotonicity of concatenation together with monotonicity and extensivity of $\rho_{\leqslant_{L}}$ imply that the inclusion $\rho_{\leqslant_{L}}(a X) \subseteq \rho_{\leqslant_{L}}\left(a \rho_{\leqslant_{L}}(X)\right)$ holds. For the reverse inclusion, we have that:

$$
\begin{aligned}
& \rho_{\leqslant_{L}}\left(a \rho_{\leqslant_{L}}(X)\right)=\text { [By definition of } \rho_{\leqslant_{L}} \text { ] } \\
& \rho_{\leqslant_{L}}\left(\left\{a y \mid \exists x \in X, x \leqslant_{L} y\right\}\right)=\text { [By definition of } \rho_{\leqslant_{L}} \text { ] } \\
& \left\{z \mid \exists x \in X, y \in \Sigma^{*}, x \leqslant_{L} y \wedge a y \leqslant_{L} z\right\} \subseteq \text { [By monotonicity of } \leqslant_{L} \text { ] } \\
& \left\{z \mid \exists x \in X, y \in \Sigma^{*}, a x \leqslant_{L} \text { ay } \wedge \text { ay } \leqslant_{L} z\right\}=\text { [By transitivity of } \leqslant_{L} \text { ] } \\
& \left\{z \mid \exists x \in X, a x \leqslant_{L} z\right\}=\text { [By definition of } \rho_{\leqslant_{L}} \text { ] } \\
& \rho_{\leqslant}(a X) \text {. }
\end{aligned}
$$

Next, we show that if $\rho_{\leqslant_{L}}(a X)=\rho_{\leqslant_{L}}\left(a \rho_{\leqslant_{L}}(X)\right)$ for all $X \in \wp\left(\Sigma^{*}\right)$ and $a \in \Sigma$ then $\leqslant_{L}$ is left-monotone.

Let $x_{1}, x_{2} \in \Sigma^{*}$ and $a \in \sum$. If $x_{1} \leqslant_{L} x_{2}$ then $\left\{x_{2}\right\} \subseteq \rho_{\leqslant_{L}}\left(\left\{x_{1}\right\}\right)$, hence $a\left\{x_{2}\right\} \subseteq a \rho_{\leqslant_{L}}\left(\left\{x_{1}\right\}\right)$. Then, by applying the monotone function $\rho_{\leqslant_{L}}$ we have that $\rho_{\leqslant_{L}}\left(a\left\{x_{2}\right\}\right) \subseteq \rho_{\leqslant_{L}}\left(a \rho_{\leqslant_{L}}\left(\left\{x_{1}\right\}\right)\right)$, so that, by backward completeness, $\rho_{\leqslant_{L}}\left(a\left\{x_{2}\right\}\right) \subseteq \rho_{\leqslant_{L}}\left(a\left\{x_{1}\right\}\right)$. Therefore, $a\left\{x_{2}\right\} \subseteq \rho_{\leqslant_{L}}\left(a\left\{x_{1}\right\}\right)$, namely, $a x_{1} \leqslant_{L} a x_{2}$. By (4.11), this shows that $\leqslant_{L}$ is left-monotone.

Since $\rho_{\leqslant}\left(\overrightarrow{\boldsymbol{\epsilon}}^{F} \cup \operatorname{Pre}_{\mathcal{N}}(\vec{X})\right)=\rho_{\leqslant}\left(\left\lfloor\overrightarrow{\boldsymbol{\epsilon}}^{F} \cup \operatorname{Pre}_{\mathcal{N}}(\overrightarrow{\boldsymbol{X}})\right\rfloor\right)$ for every quasiorder, it follows from Lemma 4.3.2 that we can apply Theorem 4.2.10 with the abstraction $\rho_{\leqslant_{L}}$ induced by a left $L_{2}$-consistent well-quasiorder and $\mathcal{F}=\left\lfloor\vec{\epsilon}^{F} \cup \operatorname{Pre}_{\mathcal{N}}(\vec{X})\right\rfloor$ interpreted as the set of functions of the form $f_{i}=\left\lfloor\overrightarrow{\boldsymbol{\epsilon}}^{F} \cup \operatorname{Pre}_{\mathcal{N}}(\vec{X})\right\rfloor_{i}$ where each $\lfloor\cdot\rfloor_{i}$ is a function mapping each set $X \in \wp\left(\Sigma^{*}\right)$ into a minor $\lfloor X\rfloor_{i}$. Intuitively, this means that we can manipulate $\leqslant$-upward closed sets in $\wp\left(\Sigma^{*}\right)$ using their finite minors, as already shown by Abdulla et al. [1996].

As a consequence, we obtain Algorithm FAINCW which, given a left $L_{2}$ - 
consistent well-quasiorder, solves the language inclusion problem $\mathcal{L}(\mathcal{N}) \subseteq L_{2}$ for any automaton $\mathcal{N}$. The algorithm is called "word-based" because the vector $\left\langle Y_{q}\right\rangle_{q \in Q}$ consists of finite sets of words in $\Sigma^{*}$.

FAINcW: Word-based algorithm for $\mathcal{L}(\mathcal{N}) \subseteq L_{2}$

Data: NFA $\mathcal{N}=\langle Q, \Sigma, \delta, I, F\rangle$; decision procedure for $u \in L_{2}$; decidable left $L_{2}$-consistent wqo $\leqslant_{L_{2}}^{\ell}$.

$1\left\langle Y_{q}\right\rangle_{q \in Q}:=\overline{\operatorname{KLEENE}}\left(\sqsubseteq_{L_{L_{2}}^{\ell}} \cap\left(\sqsubseteq_{\leqslant_{L_{2}}^{\ell}}\right)^{-1}, \lambda \vec{X} \cdot\left\lfloor\overrightarrow{\boldsymbol{\epsilon}}^{F} \cup \operatorname{Pre}_{\mathcal{N}}(\vec{X})\right\rfloor, \vec{\varnothing}\right) ;$

2 forall $q \in I$ do

3 forall $u \in Y_{q}$ do

$4 \quad$ if $u \notin L_{2}$ then return false;

5 return true;

Theorem 4.3.3. Let $\mathcal{N}=\langle Q, \Sigma, \delta, I, F\rangle$ be an NFA and let $L_{2} \in \wp\left(\Sigma^{*}\right)$ be a language such that: (i) membership in $L_{2}$ is decidable; (ii) there exists a decidable left $L_{2}$-consistent wqo on $\Sigma^{*}$. Then, Algorithm FAINCW decides the inclusion problem $\mathcal{L}(\mathcal{N}) \subseteq L_{2}$.

Proof. Let $\leqslant_{L_{2}}^{\ell}$ be a decidable left $L_{2}$-consistent well-quasiorder on $\Sigma^{*}$. Then, we check that hypothesis (i)-(v) of Theorem 4.2.10 are satisfied.

(a) It follows from hypothesis (ii) and Lemma 4.3.2 that $\leqslant_{L_{2}}^{\ell}$ is backward complete for left concatenation and satisfies $\rho_{\leqslant_{L_{2}}^{\ell}}\left(L_{2}\right)=L_{2}$.

(b) Since $\leqslant_{L_{2}}^{\ell}$ is a wqo, then $\left\langle\left\{\rho_{\leqslant_{L_{2}}^{\ell}}(S) \mid S \in \wp\left(\Sigma^{*}\right)\right\}\right.$, $\left.\subseteq\right\rangle$ is an ACC CPO.

(c) Let $\left\lfloor\overrightarrow{\boldsymbol{\epsilon}}^{F} \cup \operatorname{Pre}_{\mathcal{N}}(\vec{X})\right\rfloor$ be the set of functions $f_{i}$ each of which maps each set $X \in \wp\left(\Sigma^{*}\right)$ into a minor of $\overrightarrow{\boldsymbol{\epsilon}}^{F} \cup \operatorname{Pre}_{\mathcal{N}}(\vec{X})$. Since $\rho_{\leqslant_{L_{2}}^{\ell}}(X)=\rho_{\leqslant_{L_{2}}^{\ell}}(\lfloor X\rfloor)$ for all $X \in \wp\left(\Sigma^{*}\right)^{|Q|}$, we have that all functions $f_{i}$ satisfy

$$
\rho_{\leqslant_{L_{2}}^{\ell}}\left(\overrightarrow{\boldsymbol{\epsilon}}^{F} \cup \operatorname{Pre}_{\mathcal{N}}(\overrightarrow{\boldsymbol{X}})\right)=\rho_{\leqslant_{L_{2}}^{\ell}}\left(f_{i}(\overrightarrow{\boldsymbol{X}})\right)
$$

(d) The equality $\rho_{\leqslant_{L_{2}}^{\ell}}\left(S_{1}\right)=\rho_{\leqslant_{L_{2}}^{\ell}}\left(S_{2}\right)$ is decidable for every $S_{1}, S_{2} \in \wp\left(\Sigma^{*}\right)^{|Q|}$ since

$$
\rho_{\leqslant_{L_{2}}^{\ell}}\left(S_{1}\right)=\rho_{\leqslant_{L_{2}}^{\ell}}\left(S_{2}\right) \Leftrightarrow S_{1} \sqsubseteq_{\leqslant_{L_{2}}^{\ell}} S_{2} \wedge S_{2} \sqsubseteq_{\leqslant_{L_{2}}^{\ell}} S_{1}
$$

and, by hypothesis (ii), $\leqslant_{L_{2}}^{\ell}$ is decidable.

(e) Since $\left.\overrightarrow{\boldsymbol{L}_{2}} I=\left\langle\psi_{\Sigma^{*}}^{L_{2}}(q \in I)\right\rangle_{q \in Q}\right)$, the inclusion trivially holds for all components $Y_{q}$ with $q \notin I$. Therefore, it suffices to check whether $Y_{q} \subseteq L_{2}$ 
holds for $q \in I$ which, since $Y_{q}=\lfloor S\rfloor$ with $S \in \wp\left(\Sigma^{*}\right)$, can be decided by performing finitely many membership tests as done by lines $2-5$ of Algorithm FAIncW. By hypothesis (i), this check is decidable.

\subsubsection{Right Concatenation}

Following Section 4.2.2.1, a symmetric version, called FAINCWr, of Algorithm FAINcW and of Theorem 4.3.3 for right $L_{2}$-consistent wqos can be easily derived as follows.

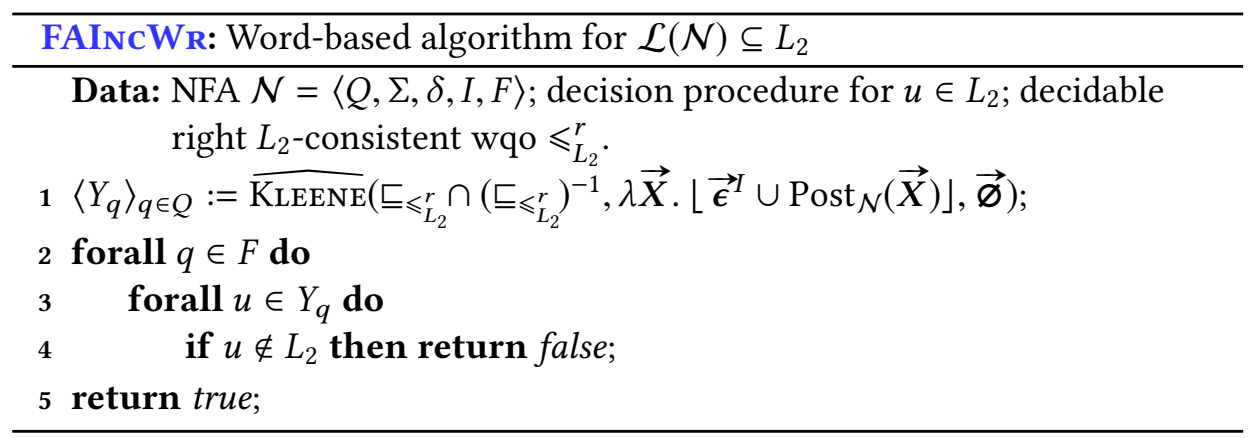

THEOREM 4.3.4. Let $\mathcal{N}=\langle Q, \Sigma, \delta, I, F\rangle$ be an NFA and let $L_{2} \in \wp\left(\Sigma^{*}\right)$ be a language such that (i) membership in $L_{2}$ is decidable; (ii) there exists a decidable right $L_{2}$-consistent wqo on $\Sigma^{*}$. Then, Algorithm FAINCWR decides the inclusion problem $\mathcal{L}(\mathcal{N}) \subseteq L_{2}$.

In the following, we will consider different quasiorders on $\Sigma^{*}$ and we will show that they fulfill the requirements of Theorem 4.3.3, so that they yield algorithms for solving a language inclusion problem.

\subsubsection{Nerode Quasiorders}

Recall from Chapter 3 that the left and right Nerode's quasiorders on $\Sigma^{*}$ are defined in the standard way:

$$
u \leqslant_{L}^{\ell} v \stackrel{\text { def }}{\Leftrightarrow} L u^{-1} \subseteq L v^{-1}, \quad u \leqslant_{L}^{r} v \stackrel{\text { def }}{\Leftrightarrow} u^{-1} L \subseteq v^{-1} L .
$$

The following result shows that Nerode's quasiorders are the weakest (i.e. greatest w.r.t. set inclusion of binary relations) $L_{2}$-consistent quasiorders for which the algorithm FAINcW can be instantiated to decide a language inclusion $\mathcal{L}(\mathcal{N}) \subseteq L_{2}$. 
LEMMA 4.3.5. Let $L \in \wp\left(\Sigma^{*}\right)$. Then

(a) $\leqslant_{L}^{\ell}$ and $\leqslant_{L}^{r}$ are, respectively, left and right $L$-consistent quasiorders. If $L$ is regular then, additionally, $\leqslant_{L}^{\ell}$ and $\leqslant_{L}^{r}$ are, respectively, decidable wqos.

(b) Let $\leqslant^{\ell}$ (resp. $\leqslant^{r}$ ) be a left (resp. right) L-consistent quasiorder on $\Sigma^{*}$. Then $\rho_{\leqslant_{L}^{\ell}} \subseteq \rho_{\leqslant \ell}\left(\right.$ resp. $\left.\rho_{\leqslant_{L}^{r}} \subseteq \rho_{\leqslant r}\right)$.

\section{Proof.}

(a) As explained in Chapter 3, de Luca and Varricchio [1994, Section 2] show that $\leqslant_{L}^{\ell}$ and $\leqslant_{L}^{r}$ are, respectively, left and right monotone quasiorders.

On the other hand, note that given $u \in L$ and $v \notin L$ we have that $\epsilon \in L u^{-1}$ and $\epsilon \in u^{-1} L$ while $\epsilon \notin L v^{-1}$ and $\epsilon \notin v^{-1} L$. Hence, $\leqslant_{L}^{\ell}$ (resp. $\leqslant_{L}^{r}$ ) is a left (resp. right) $L$-consistent quasiorder.

Finally, if $L$ is regular then both relations are clearly decidable.

(b) We consider the left case (the right case is symmetric).

As shown by de Luca and Varricchio [1994, Section 2, point 4], $\leqslant_{L}^{\ell}$ is maximum in the set of all the left $L$-consistent quasiorders, i.e. every left $L$-consistent quasiorder $\leqslant^{\ell}$ is such that $x \leqslant^{\ell} y \Rightarrow x \leqslant_{L}^{\ell} y$. As a consequence, $\rho_{\leqslant \ell}(X) \subseteq \rho_{\leqslant_{L}^{\ell}}(X)$ holds for all $X \in \wp\left(\Sigma^{*}\right)$, namely, $\leqslant^{\ell} \subseteq \leqslant_{L}^{\ell}$.

We then derive a first instantiation of Theorem 4.3.3. Because membership is decidable for regular languages $L_{2}$, Lemma 4.3 .5 (a) for $\leqslant_{L_{2}}^{\ell}$ implies that the hypotheses (i) and (ii) of Theorem 4.3.3 are satisfied, so that Algorithm FAIncW instantiated to $\leqslant_{L_{2}}^{\ell}$ decides the inclusion $\mathcal{L}(\mathcal{N}) \subseteq L_{2}$ when $L_{2}$ is regular.

Furthermore, under these hypotheses, Lemma 4.3.5 (b) shows that $\leqslant_{L_{2}}^{\ell}$ is the weakest (i.e. greatest for set inclusion) left $L_{2}$-consistent quasiorder for which the algorithm FAINcW can be instantiated for deciding the inclusion $\mathcal{L}(\mathcal{N}) \subseteq L_{2}$.

Example 4.3.6. We illustrate the use of the left Nerode's quasiorder in the algorithm FAINCW for solving the language inclusion $\mathcal{L}\left(\mathcal{N}_{1}\right) \subseteq \mathcal{L}\left(\mathcal{N}_{2}\right)$, where $\mathcal{N}_{1}$ and $\mathcal{N}_{2}$ are the automata shown in Figure 4.2. The equations for $\mathcal{N}_{1}$ are as follows:

$$
\operatorname{Eqn}\left(\mathcal{N}_{1}\right)=\left\{\begin{array}{l}
X_{1}=\varnothing \cup a X_{1} \cup a X_{2} \cup b X_{2} \cup c X_{2} \\
X_{2}=\{\epsilon\}
\end{array} .\right.
$$

We have the following quotients (among others) for $L=\mathcal{L}\left(\mathcal{N}_{2}\right)$.

$$
\begin{array}{rlrl}
L \epsilon^{-1} & =a^{*}\left(a(a+b)^{*} a+a^{+} c+a b+b b\right) & & L b^{-1}=a^{*}(a+b) \\
L a^{-1} & =a^{*} a(a+b)^{*} & L c^{-1}=a^{*} a^{+} \\
L w^{-1} & =a^{*} \text { iff } w \in\left(a(a+b)^{*} a+a c+a b+b b\right) & &
\end{array}
$$



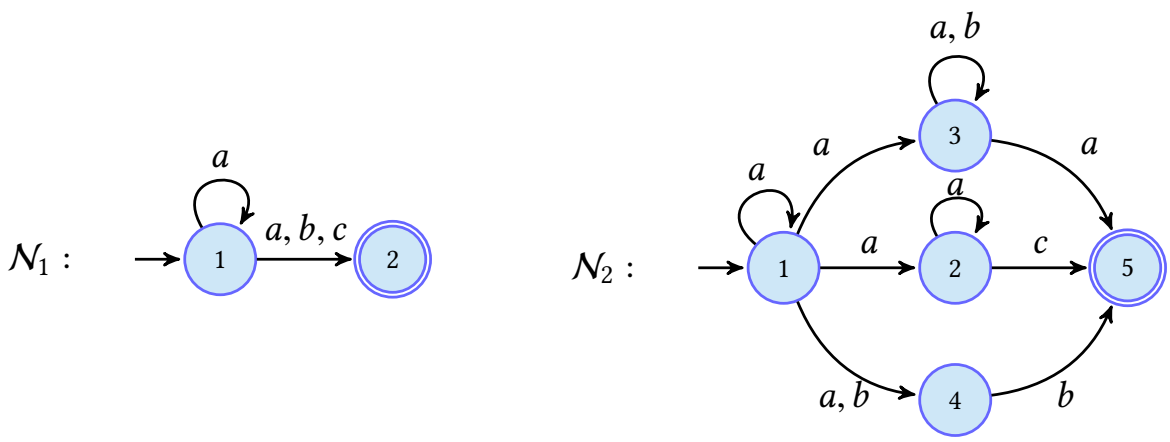

Figure 4.2: Two automata $\mathcal{N}_{1}$ (left) and $\mathcal{N}_{2}$ (right) generating the regular languages $\mathcal{L}\left(\mathcal{N}_{1}\right)=a^{*}(a+b+c)$ and $\mathcal{L}\left(\mathcal{N}_{2}\right)=a^{*}\left(a(a+b)^{*} a+a^{+} c+a b+b b\right)$.

It is straightforward to check that, among others, the following relations hold between different alphabet symbols: $b \leqslant_{L}^{\ell} a, c \leqslant_{L}^{\ell}$ a and $c \leqslant_{L}^{\ell} b$. Then, let us show the computation of the Kleene iterates performed by Algorithm FAINCW.

$$
\begin{aligned}
\vec{Y}^{(0)} & =\vec{\varnothing} \\
\vec{Y}^{(1)} & =\overrightarrow{\boldsymbol{\epsilon}}^{F}=\langle\varnothing,\{\epsilon\}\rangle \\
\vec{Y}^{(2)} & =\left\lfloor\overrightarrow{\boldsymbol{\epsilon}}^{F}\right\rfloor \sqcup\left\lfloor\operatorname{Pre}_{\mathcal{N}_{1}}\left(\vec{Y}^{(1)}\right)\right\rfloor=\langle\varnothing,\{\epsilon\}\rangle \sqcup\langle\lfloor\varnothing \cup a \varnothing \cup a\{\epsilon\} \cup b\{\epsilon\} \cup c\{\epsilon\}\rfloor,\lfloor\{\epsilon\}\rfloor\rangle \\
& =\langle\lfloor\{a, b, c\}\rfloor,\lfloor\{\epsilon\}\rfloor\rangle=\langle\{c\},\{\epsilon\}\rangle \\
\vec{Y}^{(3)} & =\left\lfloor\overrightarrow{\boldsymbol{\epsilon}}^{F}\right\rfloor \sqcup\left\lfloor\operatorname{Pre}_{\mathcal{N}_{1}}\left(\vec{Y}^{(2)}\right)\right\rfloor=\langle\varnothing,\{\epsilon\}\rangle \sqcup\langle\lfloor\varnothing \cup a\{c\} \cup a\{\epsilon\} \cup b\{\epsilon\} \cup c\{\epsilon\}\rfloor,\lfloor\{\epsilon\}\rfloor\rangle \\
& =\langle\lfloor\{a c, a, b, c\}\rfloor,\lfloor\{\epsilon\}\rfloor\rangle=\langle\{c\},\{\epsilon\}\rangle
\end{aligned}
$$

The least fixpoint is thus $\vec{Y}=\langle\{c\},\{\epsilon\}\rangle$. Since $c \in \vec{Y}_{1}$ and $c \notin \mathcal{L}\left(\mathcal{N}_{2}\right)$, Algorithm FAINCW concludes that the language inclusion $\mathcal{L}\left(\mathcal{N}_{1}\right) \subseteq \mathcal{L}\left(\mathcal{N}_{2}\right)$ does not hold. $\diamond$

\subsubsection{On the Complexity of Nerode's quasiorders}

For the inclusion problem between languages generated by finite automata, deciding the (left or right) Nerode's quasiorder can be easily shown to be as hard as the language inclusion problem, which is PSPACE-complete. In fact, given the automata $\left.\mathcal{N}_{1}=\left\langle Q_{1}, \delta_{1}, I_{1}, F_{1}, \Sigma\right)\right\rangle$ and $\mathcal{N}_{2}=\left\langle Q_{2}, \delta_{2}, I_{2}, F_{2}, \Sigma\right\rangle$, one can define the union automaton $\mathcal{N}_{3} \stackrel{\text { def }}{=}\left\langle Q_{1} \cup Q_{2} \cup\left\{q^{l}\right\}, \delta_{3},\left\{q^{l}\right\}, F_{1} \cup F_{2}\right\rangle$ where $\delta_{3}$ maps $\left(q^{l}, a\right)$ to $I_{1},\left(q^{l}, b\right)$ to $I_{2}$ and behaves like $\delta_{1}$ or $\delta_{2}$ elsewhere. Then, it turns out that

$$
a \leqslant_{{ }^{r}\left(\mathcal{N}_{3}\right)} b \Leftrightarrow a^{-1} \mathcal{L}\left(\mathcal{N}_{3}\right) \subseteq b^{-1} \mathcal{L}\left(\mathcal{N}_{3}\right) \Leftrightarrow \mathcal{L}\left(\mathcal{N}_{1}\right) \subseteq \mathcal{L}\left(\mathcal{N}_{2}\right) .
$$

It follows that deciding the right Nerode's quasiorder $\leqslant_{\mathcal{L}\left(N_{3}\right)}^{r}$ is as hard as deciding $\mathcal{L}\left(\mathcal{N}_{1}\right) \subseteq \mathcal{L}\left(\mathcal{N}_{2}\right)$. 
Also, for the inclusion problem of a language generated by an automaton within the trace set of a one-counter net (see Section 4.3.4), the right Nerode's quasiorder is a right language-consistent well-quasiorder but it turns out to be undecidable (see Lemma 4.3.13).

\subsubsection{State-based Quasiorders}

Consider the inclusion problem $\mathcal{L}\left(\mathcal{N}_{1}\right) \subseteq \mathcal{L}\left(\mathcal{N}_{2}\right)$ where $\mathcal{N}_{1}$ and $\mathcal{N}_{2}$ are NFAs. In the following, we study a class of well-quasiorders based on $\mathcal{N}_{2}$, called statebased quasiorders. These quasiorders are strictly stronger (i.e. lower w.r.t. set inclusion of binary relations) than the Nerode's quasiorders and sidestep the untractability or undecidability of Nerode's quasiorders yet allowing to define an algorithm solving the language inclusion problem.

\subsubsection{Inclusion in Regular Languages.}

We define the quasiorders $\leqslant_{\mathcal{N}}^{\ell}$ and $\leqslant_{\mathcal{N}}^{r}$ induced by an NFA $\mathcal{N}=\langle Q, \Sigma, \delta, I, F\rangle$ as follows:

$$
u \leqslant_{\mathcal{N}}^{\ell} v \stackrel{\text { def }}{\Leftrightarrow} \operatorname{pre}_{u}^{\mathcal{N}}(F) \subseteq \operatorname{pre}_{v}^{\mathcal{N}}(F), \quad u \leqslant_{\mathcal{N}}^{r} v \stackrel{\text { def }}{\Leftrightarrow} \operatorname{post}_{u}^{\mathcal{N}}(I) \subseteq \operatorname{post}_{v}^{\mathcal{N}}(I) .
$$

The superscripts in $\leqslant_{\mathcal{N}}^{\ell}$ and $\leqslant_{\mathcal{N}}^{r}$ stand, respectively, for left/right because they are, respectively, left and right well-quasiorders as the following result shows.

LЕМмА 4.3.7. The relations $\leqslant_{\mathcal{N}}^{\ell}$ and $\leqslant_{\mathcal{N}}^{r}$ are, respectively, decidable left and right $\mathcal{L}(\mathcal{N})$-consistent wqos.

Proof. Since, for every $u \in \Sigma^{*}, \operatorname{pre}_{u}^{\mathcal{N}}(F)$ is a computable subset of a the finite set of states of $\mathcal{N}$, it turns out that $\leqslant_{N}^{\ell}$ is a decidable wqo. Let us check that $\leqslant_{\mathcal{N}}^{\ell}$ is left $\mathcal{L}(\mathcal{N})$-consistent according to Definition 4.3.1 (a)-(b).

(a) Let $u \in \mathcal{L}(\mathcal{N})$ and $v \notin \mathcal{L}(\mathcal{N})$. We have that $\operatorname{pre}_{u}^{\mathcal{N}}(F)$ contains some initial state while $\operatorname{pre}_{v}^{\mathcal{N}}(F)$ does not, hence $u \not_{\mathcal{N}}^{\ell} v$. Therefore, $\leqslant_{\mathcal{N}}^{\ell} \cap\left(L \times L^{c}\right)=\varnothing$.

(b) Let us check that $\leqslant_{\mathcal{N}}^{\ell}$ is left monotone. Observe that $\operatorname{pre}_{x}^{\mathcal{N}}$ is a monotone function and that

$$
\operatorname{pre}_{u v}^{\mathcal{N}}=\operatorname{pre}_{u}^{\mathcal{N}} \circ \operatorname{pre}_{v}^{\mathcal{N}} .
$$

Therefore, for all $x_{1}, x_{2} \in \Sigma^{*}$ and $a \in \Sigma$,

$$
\begin{aligned}
x_{1} \leqslant_{\mathcal{N}}^{\ell} x_{2} \Rightarrow & {\left[\text { By definition of } \leqslant_{\mathcal{N}}^{\ell}\right] } \\
\operatorname{pre}_{x_{1}}^{\mathcal{N}}(F) \subseteq \operatorname{pre}_{x_{2}}^{\mathcal{N}}(F) \Rightarrow & {\left[\text { Since pre }{ }_{a}^{\mathcal{N}} \text { is monotone }\right] }
\end{aligned}
$$




$$
\begin{array}{rc}
\operatorname{pre}_{a}^{\mathcal{N}}\left(\operatorname{pre}_{x_{1}}^{\mathcal{N}}(F)\right) \subseteq \operatorname{pre}_{a}^{\mathcal{N}}\left(\operatorname{pre}_{x_{2}}^{\mathcal{N}}(F)\right) \Leftrightarrow & {[\text { By Equation (4.13)] }} \\
\operatorname{pre}_{a x_{1}}^{\mathcal{N}}(F) \subseteq \operatorname{pre}_{a x_{2}}^{\mathcal{N}}(F) \Leftrightarrow & {\left[\text { By definition of } \leqslant_{\mathcal{N}}^{\ell}\right]} \\
a x_{1} \leqslant_{\mathcal{N}}^{\ell} a x_{2} &
\end{array}
$$

The proof that $\leq_{\mathcal{N}}^{r}$ is a decidable right $\mathcal{L}(\mathcal{N})$-consistent quasiorder is symmetric.

As a consequence, Theorem 4.3.3 applies to the wqo $\leqslant_{\mathcal{N}_{2}}^{\ell}$ (and $\leqslant_{\mathcal{N}_{2}}^{r}$ ), so that one can instantiate Algorithm FAINCW with $\leqslant_{\mathcal{N}_{2}}^{\ell}$ for deciding $\mathcal{L}\left(\mathcal{N}_{1}\right) \subseteq \mathcal{L}\left(\mathcal{N}_{2}\right)$.

Turning back to the left Nerode wqo $\leqslant_{\mathcal{L}\left(\mathcal{N}_{2}\right)}^{\ell}$, it turns out that:

$$
u \leqslant_{\mathcal{L}\left(\mathcal{N}_{2}\right)}^{\ell} v \Leftrightarrow \mathcal{L}\left(\mathcal{N}_{2}\right) u^{-1} \subseteq \mathcal{L}\left(\mathcal{N}_{2}\right) v^{-1} \Leftrightarrow W_{I, \operatorname{pre}_{u}^{\mathcal{N}_{2}}(F)} \subseteq W_{I, \operatorname{pre}_{v}^{\mathcal{N}_{2}}(F)} .
$$

Since $\operatorname{pre}_{u}^{\mathcal{N}_{2}}(F) \subseteq \operatorname{pre}_{v}^{\mathcal{N}_{2}}(F) \Rightarrow W_{I, \operatorname{pre}_{u}^{\mathcal{N}_{2}}(F)} \subseteq W_{I, \operatorname{pre}_{v}^{\mathcal{N}_{2}}(F)}$, it follows that

$$
u \leqslant_{\mathcal{N}_{2}}^{\ell} v \Rightarrow u \leqslant_{\mathcal{L}\left(\mathcal{N}_{2}\right)}^{\ell} v
$$

Example 4.3.8. We illustrate the left state-based quasiorder by using it to solve the language inclusion $\mathcal{L}\left(\mathcal{N}_{1}\right) \subseteq \mathcal{L}\left(\mathcal{N}_{2}\right)$ from Example 4.3.6. We have, among others, the following set of predecessors of $F_{\mathcal{N}_{2}}$ :

$$
\begin{array}{llll}
\operatorname{pre}_{\epsilon}^{\mathcal{N}_{2}}\left(F_{\mathcal{N}_{2}}\right)=\{5\} & \operatorname{pre}_{a}^{\mathcal{N}_{2}}\left(F_{\mathcal{N}_{2}}\right)=\{3\} & \operatorname{pre}_{b}^{\mathcal{N}_{2}}\left(F_{\mathcal{N}_{2}}\right)=\{4\} & \operatorname{pre}_{c}^{\mathcal{N}_{2}}\left(F_{\mathcal{N}_{2}}\right)=\{2\} \\
\operatorname{pre}_{a a}^{\mathcal{N}_{2}}\left(F_{\mathcal{N}_{2}}\right)=\{1,3\} & \operatorname{pre}_{a b}^{\mathcal{N}_{2}}\left(F_{\mathcal{N}_{2}}\right)=\{1\} & \operatorname{pre}_{a c}^{\mathcal{N}_{2}}\left(F_{\mathcal{N}_{2}}\right)=\{1,2\} & \operatorname{pre}_{a a b}^{\mathcal{N}_{2}}\left(F_{\mathcal{N}_{2}}\right)=\{1\}
\end{array}
$$

Recall from Example 4.3.6 that, for the Nerode's quasiorder, we have that $b \leqslant_{\mathcal{L}\left(\mathcal{N}_{2}\right)}^{\ell}$ a and $c \leqslant_{\mathcal{L}\left(\mathcal{N}_{2}\right)}^{\ell}$ a while none of these relations hold for $\leqslant_{\mathcal{N}_{2}}^{\ell}$. Let us next show the Kleene iterates computed by Algorithm FAINCW when using $\leqslant_{\mathcal{N}_{2}}^{\ell}$.

$$
\begin{aligned}
\vec{Y}^{(0)} & =\vec{\varnothing} \\
\vec{Y}^{(1)} & =\overrightarrow{\boldsymbol{\epsilon}}^{F}=\langle\varnothing,\{\epsilon\}\rangle \\
\vec{Y}^{(2)} & =\left\lfloor\overrightarrow{\boldsymbol{\epsilon}}^{F}\right\rfloor \sqcup\left\lfloor\operatorname{Pre}_{\mathcal{N}_{1}}\left(\vec{Y}^{(1)}\right)\right\rfloor=\langle\lfloor\{a, b, c\}\rfloor,\lfloor\{\epsilon\}\rfloor\rangle=\langle\{a, b, c\},\{\epsilon\}\rangle \\
\vec{Y}^{(3)} & =\left\lfloor\overrightarrow{\boldsymbol{\epsilon}}^{F}\right\rfloor \sqcup\left\lfloor\operatorname{Pre}_{\mathcal{N}_{1}}\left(\vec{Y}^{(2)}\right)\right\rfloor=\langle\lfloor\{a a, a b, a c, a, b, c\}\rfloor,\lfloor\{\epsilon\}\rfloor\rangle=\langle\{a b, a, b, c\},\{\epsilon\}\rangle \\
\vec{Y}^{(4)} & =\left\lfloor\overrightarrow{\boldsymbol{\epsilon}}^{F}\right\rfloor \sqcup\left\lfloor\operatorname{Pre}_{\mathcal{N}_{1}}\left(\vec{Y}^{(3)}\right)\right\rfloor=\langle\lfloor\{a a b, a a, a b, a c, a, b, c\}\rfloor,\lfloor\{\epsilon\}\rfloor\rangle \\
& =\langle\{a b, a, b, c\},\{\epsilon\}\rangle
\end{aligned}
$$

The least fixpoint is therefore $\vec{Y}=\langle\{a b, a, b, c\},\{\epsilon\}\rangle$. Since $c \in \vec{Y}_{0}$ and $c \notin \mathcal{L}\left(\mathcal{N}_{2}\right)$, Algorithm FAINCW concludes that the inclusion $\mathcal{L}\left(\mathcal{N}_{1}\right) \subseteq \mathcal{L}\left(\mathcal{N}_{2}\right)$ does not hold. $\diamond$ 


\subsubsection{Simulation-based Quasiorders.}

Recall that a simulation on an NFA $\mathcal{N}=\langle Q, \Sigma, \delta, I, F\rangle$ is a binary relation on the states of $\mathcal{N}$, i.e. $\leq \subseteq Q \times Q$, such that for all $p, q \in Q$ if $p \leq q$ then the following two conditions hold:

(i) if $p \in F$ then $q \in F$;

(ii) for every transition $p \stackrel{a}{\rightarrow} p^{\prime}$, there exists a transition $q \stackrel{a}{\rightarrow} q^{\prime}$ such that $p^{\prime} \leq q^{\prime}$.

It is well known that simulation implies language inclusion, i.e. if $\leq$ is a simulation on $\mathcal{N}$ then

$$
q \leq q^{\prime} \Rightarrow W_{q, F}^{\mathcal{N}} \subseteq W_{q^{\prime}, F}^{\mathcal{N}} .
$$

A relation $\leq \subseteq Q \times Q$ can be lifted in the standard universal-existential way to a relation $\leq^{\forall \exists} \subseteq \wp(Q) \times \wp(Q)$ on sets of states as follows:

$$
X \leq^{\forall \exists} Y \stackrel{\text { def }}{\Leftrightarrow} \forall x \in X, \exists y \in Y, x \leq y .
$$

In particular, if $\leq$ is a qo then $\leq^{\forall \exists}$ is a qo as well. Also, if $\leq$ is a simulation relation then its lifting $\leq^{\forall \exists}$ is such that $X \leq^{\forall \exists} Y \Rightarrow W_{X, F}^{\mathcal{N}} \subseteq W_{Y, F}^{\mathcal{N}}$ holds. This suggests us to define a right simulation-based quasiorder $\leq_{\mathcal{N}}^{r}$ on $\Sigma^{*}$ induced by a simulation $\leq$ on $\mathcal{N}$ as follows:

$$
u \leq_{\mathcal{N}}^{r} v \stackrel{\text { def }}{\Leftrightarrow} \operatorname{post}_{u}^{\mathcal{N}}(I) \leq^{\forall \exists} \operatorname{post}_{v}^{\mathcal{N}}(I) .
$$

LемMA 4.3.9. Let $\mathcal{N}$ be an NFA and let $\leq$ be a simulation on $\mathcal{N}$. Then the right simulation-based quasiorder $\leq_{\mathcal{N}}^{r}$ is a decidable right $\mathcal{L}(\mathcal{N})$-consistent well-quasiorder.

Proof. Since, for every $u \in \Sigma^{*}, \operatorname{post}_{u}^{\mathcal{N}}(F)$ is a computable subset of a the finite set of states of $\mathcal{N}$, it turns out that $\leq_{\mathcal{N}}^{r}$ is a decidable wqo. Next, we show that $\leq_{\mathcal{N}}^{r}$ is right $\mathcal{L}(\mathcal{N})$-consistent according to Definition 4.3.1 (a)-(b).

(a) Let $u \in \mathcal{L}(\mathcal{N})$ and $v \notin \mathcal{L}(\mathcal{N})$. We have that $\operatorname{post}_{u}^{\mathcal{N}}(I)$ contains some final state while $\operatorname{post}_{v}^{\mathcal{N}}(I)$ does not. Let $q \in \operatorname{post}_{u}^{\mathcal{N}}(I) \cap F$. We have that $q \leq_{\mathcal{N}}^{r} q^{\prime}$ for no $q^{\prime} \in \operatorname{post}_{v}^{\mathcal{N}}(I)$ since, by simulation, this would imply $q^{\prime} \in \operatorname{post}_{v}^{\mathcal{N}}(I) \cap F$, which contradicts the fact that $F \cap \operatorname{post}_{v}^{\mathcal{N}}(I)=\varnothing$. We conclude that $u \npreceq_{\mathcal{N}}^{r} v$, hence $\leq_{\mathcal{N}}^{r} \cap\left(L \times L^{c}\right)=\varnothing$.

(b) Next we show that $\leq_{\mathcal{N}}^{r}$ is right monotone. By Equation (4.11), we check that for all $u, v \in \Sigma^{*}$ and $a \in \Sigma, u \leq_{\mathcal{N}}^{r} v \Rightarrow u a \leq_{\mathcal{N}}^{r} v a$ :

$$
\begin{aligned}
u \leq_{\mathcal{N}}^{r} v & \Leftrightarrow\left[\text { By def. of } \leq_{\mathcal{N}}^{r}\right] \\
\operatorname{post}_{u}^{\mathcal{N}}(I) \leq^{\forall \exists} \operatorname{post}_{v}^{\mathcal{N}}(I) & \Leftrightarrow\left[\text { By def. of } \leq^{\forall \exists}\right] \\
\forall x \in \operatorname{post}_{u}^{\mathcal{N}}(I), \exists y \in \operatorname{post}_{v}^{\mathcal{N}}(I), x \leq y & \Rightarrow[\text { By def. of } \leq]
\end{aligned}
$$




$$
\begin{array}{r}
\forall x \stackrel{a}{\rightarrow} x^{\prime}, x \in \operatorname{post}_{u}^{\mathcal{N}}(I), \exists y \stackrel{a}{\rightarrow} y^{\prime}, y \in \operatorname{post}_{v}^{\mathcal{N}}(u), x^{\prime} \leq y^{\prime} \Leftrightarrow \\
\quad\left[\text { Since post }{ }_{a}^{\mathcal{N}} \circ \operatorname{post}_{u}^{\mathcal{N}}=\text { post }_{u a}^{\mathcal{N}}(I)\right] \\
\forall x^{\prime} \in \operatorname{post}_{u a}^{\mathcal{N}}(I), \exists y^{\prime} \in \operatorname{post}_{v a}^{\mathcal{N}}(I), x^{\prime} \leq y^{\prime} \Leftrightarrow\left[\text { By def. of } \leq^{\forall \exists}\right. \text { ] } \\
\operatorname{post}_{u a}^{\mathcal{N}}(I) \leq^{\forall \exists} \text { post }_{v a}^{\mathcal{N}}(I) \Leftrightarrow \text { [By def. of } \leq_{\mathcal{N}}^{r} \text { ] } \\
u a \leq_{\mathcal{N}}^{r} v a .
\end{array}
$$

Thus, once again, Theorem 4.3.4 applies to $\preceq_{\mathcal{N}_{2}}^{r}$ and this allows us to instantiate the algorithm FAINCWR to the quasiorder $\leq_{\mathcal{N}_{2}}^{r}$ for deciding the inclusion $\mathcal{L}\left(\mathcal{N}_{1}\right) \subseteq \mathcal{L}\left(\mathcal{N}_{2}\right)$.

On the other hand, note that it is possible to define a left simulation $\preceq_{R}^{\forall \exists}$ on an automaton $\mathcal{N}$ by applying $\leq^{\forall \exists}$ on the reverse of $\mathcal{N}$. This left simulation induces a left simulation-based quasiorder on $\Sigma^{*}$ as follows:

$$
u \preceq_{\mathcal{N}}^{l} v \stackrel{\text { def }}{\Leftrightarrow} \operatorname{pre}_{u}^{\mathcal{N}}(F) \preceq_{R}^{\forall \exists} \operatorname{pre}_{v}^{\mathcal{N}}(F)
$$

It is straightforward to check that Theorem 4.3.3 applies to $\leq_{\mathcal{N}_{2}}^{\ell}$ and, therefore, we can instantiate Algorithm FAINcW for deciding $\mathcal{L}\left(\mathcal{N}_{1}\right) \subseteq \mathcal{L}\left(\mathcal{N}_{2}\right)$.

Example 4.3.10. Finally, let us illustrate the use of the left simulation-based quasiorder to solve the language inclusion $\mathcal{L}\left(\mathcal{N}_{1}\right) \subseteq \mathcal{L}\left(\mathcal{N}_{2}\right)$ of Example 4.3.6. For the set $F_{\mathcal{N}_{2}}$ of final states $\mathcal{N}_{2}$ we have the same set of predecessors computed in Example 4.3.8 and, among others, the following left simulations between these sets (For clarity, we omit the argument of the function pre, which is always $F_{\mathcal{N}_{2}}$ ):

$$
\begin{array}{ll}
\operatorname{pre}_{c}^{\mathcal{N}_{2}}()=\{2\} \preceq_{R}^{\forall \exists}\{3\}=\operatorname{pre}_{a}^{\mathcal{N}_{2}}() & \operatorname{pre}_{b}^{\mathcal{N}_{2}}()=\{4\} \not_{R}^{\forall \exists}\{3\}=\operatorname{pre}_{a}^{\mathcal{N}_{2}}() \\
\operatorname{pre}_{a c}^{\mathcal{N}_{2}}()=\{1\} \preceq_{R}^{\forall \exists}\{4\}=\operatorname{pre}_{b}^{\mathcal{N}_{2}}() & \operatorname{pre}_{a c}^{\mathcal{N}_{2}}()=\{1\} \not_{R}^{\forall \exists}\{2\}=\operatorname{pre}_{c}^{\mathcal{N}_{2}}()
\end{array}
$$

As expected, the simulation-based quasiorder lies in between the Nerode and the state-based quasiorders. As shown in Examples 4.3.6 and 4.3.8, we have $b \leqslant_{\mathcal{L}\left(N_{2}\right)}^{\ell}$, $c \leqslant_{\mathcal{L}\left(\mathcal{N}_{2}\right)}^{\ell} a, b \Varangle_{\mathcal{N}_{2}}^{\ell}$ a and $c \Varangle_{\mathcal{N}_{2}}^{\ell}$ a while $c \leq_{\mathcal{N}_{2}}^{\ell}$ a, but $b \Varangle_{\mathcal{N}_{2}}^{\ell}$ a.

Let us show the computation of the Kleene iterates performed by Algorithm FAINCW when using the quasiorder $\leq_{\mathcal{N}_{2}}^{\ell}$.

$$
\begin{aligned}
& \vec{Y}^{(0)}=\vec{\varnothing} \\
& \vec{Y}^{(1)}=\overrightarrow{\boldsymbol{\epsilon}}^{F}=\langle\varnothing,\{\epsilon\}\rangle \\
& \vec{Y}^{(2)}=\left\lfloor\overrightarrow{\boldsymbol{\epsilon}}^{F}\right\rfloor \sqcup\left\lfloor\operatorname{Pre}_{\mathcal{N}_{1}}\left(\vec{Y}^{(1)}\right)\right\rfloor=\langle\lfloor\{a, b, c\}\rfloor,\lfloor\{\varepsilon\}\rfloor\rangle=\langle\{c\},\{\varepsilon\}\rangle \\
& \vec{Y}^{(3)}=\left\lfloor\overrightarrow{\boldsymbol{\epsilon}}^{F}\right\rfloor \sqcup\left\lfloor\operatorname{Pre}_{\mathcal{N}_{1}}\left(\vec{Y}^{(2)}\right)\right\rfloor=\langle\lfloor\{a c, a, b, c\}\rfloor,\lfloor\{\varepsilon\}\rfloor\rangle=\langle\{c\},\{\varepsilon\}\rangle
\end{aligned}
$$

The least fixpoint is therefore $\vec{Y}=\langle\{c\},\{\varepsilon\}\rangle$. Since $c \in \vec{Y}_{0}$ and $c \notin \mathcal{L}\left(\mathcal{N}_{2}\right)$, Algorithm FAINCW concludes that the inclusion $\mathcal{L}\left(\mathcal{N}_{1}\right) \subseteq \mathcal{L}\left(\mathcal{N}_{2}\right)$ does not hold. $\diamond$ 


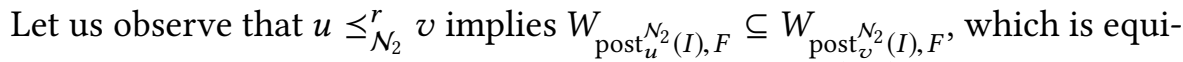
valent to the right Nerode's quasiorder $u \leqslant^{r}{ }_{\mathcal{L}\left(\mathcal{N}_{2}\right)} v$ for $\mathcal{L}\left(\mathcal{N}_{2}\right)$. Furthermore, for the state-based quasiorder defined in (4.12), we have that $u \leqslant_{\mathcal{N}_{2}}^{r} v \Rightarrow u \leq_{\mathcal{N}_{2}}^{r} v$ trivially holds.

Summing up, given an NFA $\mathcal{N}$ with $\mathcal{L}(\mathcal{N})=L$, the following containments relate the state-based, simulation-based and Nerode's quasiorders:

$$
\leqslant_{\mathcal{N}}^{r} \subseteq \leq_{\mathcal{N}}^{r} \subseteq \leqslant_{L}^{r}, \quad \leqslant_{\mathcal{N}}^{\ell} \subseteq \leq_{\mathcal{N}}^{\ell} \subseteq \leqslant_{L}^{\ell} .
$$

Recall that these are decidable $\mathcal{L}\left(\mathcal{N}_{2}\right)$-consistent well-quasiorders so that $\mathrm{Al}$ gorithm FAINcW can be instantiated for each of them for deciding an inclusion $\mathcal{L}\left(\mathcal{N}_{1}\right) \subseteq \mathcal{L}\left(\mathcal{N}_{2}\right)$. Examples 4.3.6, 4.3.8 and 4.3.10 show how the algorithm behaves for each of the three quasiorders considered in this section. Despite their simplicity, the examples evidence the differences in the behavior of the algorithm when considering the different quasiorders. In particular, we observe that the fixpoint computation for $\leqslant_{\mathcal{L}\left(\mathcal{N}_{2}\right)}^{r}$ coincides with the one for $\leq_{\mathcal{N}_{2}}^{r}$ which, as expected, converge faster than the one for $\leqslant_{\mathcal{N}_{2}}^{r}$.

As shown by de Luca and Varricchio [1994], $\leqslant_{\mathcal{L}\left(\mathcal{N}_{2}\right)}^{r}$ is the coarsest wellquasiorder for which Algorithm FAIncW works (i.e. Theorem 4.3.3 holds), hence its corresponding fixpoint computation exhibits optimal behavior in terms of the number of closed sets considered. However, Nerode's quasiorder is not practical since it requires checking language inclusion, which is the PSPACE-complete problem we are trying to solve, in order to decide whether two words are related. Therefore, the coincidence of the fixpoint computations for $\leqslant_{\mathcal{L}\left(\mathcal{N}_{2}\right)}^{r}$ and $\leq_{\mathcal{N}_{2}}^{r}$ is of special interest since it evidences that Algorithm FAINCW might exhibit optimal behavior while using a "simpler" well-quasiorder such as $\leq_{N_{2}}^{r}$, which is a polynomial under-approximation of $\leqslant_{\mathcal{L}\left(\mathcal{N}_{2}\right)}^{r}$.

\subsubsection{Inclusion in Traces of One-Counter Nets.}

We show that our framework can be instantiated to systematically derive an algorithm for deciding the inclusion $\mathcal{L}(\mathcal{N}) \subseteq L_{2}$ where $L_{2}$ is the trace set of a one-counter net. This is accomplished by defining a decidable $L_{2}$-consistent quasiorder so that Theorem 4.3.3 can be applied.

Intuitively, a one-counter net is an NFA endowed with a nonnegative integer counter which can be incremented, decremented or left unchanged by a transition.

Definition (One-Counter Net). A One-Counter Net (OCN) [Hofman and Totzke 2018] is a tuple $O=\langle Q, \Sigma, \delta\rangle$ where $Q$ is a finite set of states, $\Sigma$ is an alphabet and $\delta \subseteq Q \times \Sigma \times\{-1,0,1\} \times Q$ is a set of transitions. 
A configuration of an $O C N O=\langle Q, \Sigma, \delta\rangle$ is a pair $q n$ consisting of a state $q \in Q$ and a value $n \in \mathbb{N}$ for the counter. Given two configurations of an OCN, $q n, q^{\prime} n^{\prime} \in Q \times \mathbb{N}$, we write $q n \stackrel{a}{\rightarrow} q^{\prime} n^{\prime}$ and call it an $a$-step (or simply step) if there exists a transition $\left(q, a, d, q^{\prime}\right) \in \delta$ such that $n^{\prime}=n+d$. Given $q n \in Q \times \mathbb{N}$, the trace set $T(q n) \subseteq \Sigma^{*}$ of an OCN is defined as follows:

$$
\begin{aligned}
& T(q n) \stackrel{\text { def }}{=}\left\{u \in \Sigma^{*} \mid Z_{u}^{q n} \neq \varnothing\right\} \quad \text { where } \\
& Z_{u}^{q n} \stackrel{\text { def }}{=}\left\{q_{k} n_{k} \in Q \times \mathbb{N} \mid q n=q_{0} n_{0} \stackrel{a_{1}}{\longrightarrow} q_{1} n_{1} \stackrel{a_{2}}{\longrightarrow} \cdots \stackrel{a_{k}}{\longrightarrow} q_{k} n_{k}, a_{1} \cdots a_{k}=u\right\} .
\end{aligned}
$$

Observe that $Z_{\epsilon}^{q n}=\{q n\}$ and $Z_{u}^{q n}$ is a finite set for every word $u \in \Sigma^{*}$.

Let us consider the poset $\left\langle\mathbb{N}_{\perp} \stackrel{\text { def }}{=} \mathbb{N} \cup\{\perp\}, \leq_{\mathbb{N}_{\perp}}\right\rangle$ where $\perp \leq_{\mathbb{N}_{\perp}} n$ holds for all $n \in \mathbb{N}_{\perp}$, while for all $n, n^{\prime} \in \mathbb{N}, n \leq_{\mathbb{N}_{\perp}} n^{\prime}$ is the standard ordering relation between numbers. For a finite set of states $S \subseteq Q \times \mathbb{N}$ define the so-called macro state $M_{S}: Q \rightarrow \mathbb{N}_{\perp}$ as follows:

$$
M_{S}(q) \stackrel{\text { def }}{=} \max \{n \in \mathbb{N} \mid q n \in S\},
$$

where $\max \varnothing \stackrel{\text { def }}{=} \perp$. Define the following quasiorder on $\Sigma^{*}$ :

$$
u \leq_{q n}^{r} v \stackrel{\text { def }}{\Leftrightarrow} \forall q \in Q, M_{Z_{u}^{q n}}(q) \leq_{\mathbb{N}_{\perp}} M_{Z_{v}^{q n}}(q) .
$$

LЕMмA 4.3.11. Let $O$ be an OCN. For any configuration $q$ of $O, \leq_{q n}^{r}$ is a right $T(q n)$-consistent decidable well-quasiorder.

Proof. It follows from Dickson's Lemma [Sakarovitch 2009, Section II.7.1.2] that $\leq_{q n}^{r}$ is a wqo. Next, we show that $\leq_{q n}^{r}$ is $T(q n)$-consistent according to Definition 4.3.1 (a)-(b).

(a) Since $Z_{u}^{q n}$ and $Z_{v}^{q n}$ are finite sets, we have that the macro state functions $M_{Z_{u}^{q n}}$ and $M_{Z_{v}^{q n}}$ are computable, hence the relation $\leq_{q n}^{r}$ is decidable. Let $u \in T(q n)$ and $v \notin T(q n)$. Then $M_{Z_{u}^{q n}}\left(q^{\prime}\right) \neq \perp$ for some $q^{\prime} \in Q$ and $M_{Z_{v}^{q n}}\left(q^{\prime}\right)=\perp$ since $Z_{v}^{q n}=\varnothing$. It follows that $u{\underset{一}{q n}}_{q n}^{r} v$ and, therefore, $\leq_{q n}^{r} \cap\left(T(q n) \times(T(q n))^{c}\right)=\varnothing$.

(b) Next we show that $u \leq_{q n}^{r} v$ implies $u a \leq_{q n}^{r} v a$ for all $a \in \Sigma$, since, by Equation (4.11), this is equivalent to the fact that $\leq_{q n}^{r}$ is right monotone. We proceed by contradiction.

Assume that $u \leq_{q n}^{r} v$ and $\exists q^{\prime} \in Q, M_{Z_{u a}^{q n}}\left(q^{\prime}\right){\mathbb{N}_{\perp}}_{\mathbb{N}} M_{Z_{v a}^{q n}}\left(q^{\prime}\right)$. Then we have that $m_{1} \stackrel{\text { def }}{=} \max \left\{n \mid p n \in Z_{u a}^{q n}\right\} \mathbb{Z}_{\mathbb{N}_{\perp}} m_{2} \stackrel{\text { def }}{=} \max \left\{n \mid p n \in Z_{v a}^{q n}\right\}$, which implies, since $m_{1} \neq \perp$, that $m_{1}, m_{2} \in \mathbb{N}$ and $m_{1}>m_{2}$.

On the other hand, for all $\left(q, a, d, q^{\prime}\right) \in \delta$ we have $q^{\prime}\left(m_{1}-d\right) \in Z_{u}^{q n}$ and $q^{\prime}\left(m_{2}-d\right) \in Z_{v}^{q n}$. 
Observe that $\max \left\{n \mid p n \in Z_{u}^{q n}\right\}=m_{1}-d$ since otherwise we would that have $\max \left\{n \mid p n \in Z_{u}^{q n}\right\}+d>m_{1}$ which contradicts the definition of $m_{1}$. Similarly, $\max \left\{n \mid p n \in Z_{v}^{q n}\right\}=m_{2}-d$.

Since $m_{1}>m_{2}$ we have that $m_{1}-d>m_{2}-d$ and, as a consequence, $\max \left\{n \mid p n \in Z_{u}^{q n}\right\}>\max \left\{n \mid p n \in Z_{v}^{q n}\right\}$, which contradicts $u \leq_{q n}^{r} v$.

Thus, as a consequence of Theorem 4.3.3, Lemma 4.3.11 and the decidability of membership $u \in T(q n)$, the following known decidability result for language inclusion of regular languages into traces of OCNs [Jancar et al. 1999, Theorem $3.2]$ is systematically derived within our framework.

COROLlARY 4.3.12. Let $\mathcal{N}$ be an NFA and $O$ be an OCN. For any configuration qn of $O$, the language inclusion $\mathcal{L}(\mathcal{N}) \subseteq T(q n)$ is decidable.

Moreover, the following result closes a conjecture made by de Luca and Varricchio [1994, Section 6].

LEMMA 4.3.13. Let $O$ be an OCN. Then the right Nerode's quasiorder $\leqslant_{T(q n)}^{r}$ is an undecidable well-quasiorder.

Proof. Recall that $\leqslant_{T(q n)}^{r}$ is maximum in the set of all right $T(q n)$-consistent quasiorders [de Luca and Varricchio 1994, Section 2, point 4]. As a consequence, $u \leqslant_{q n}^{r} v \Rightarrow u \leqslant_{T(q n)}^{r} v$, for all $u, v \in \Sigma^{*}$. By Lemma 4.3.11, $\leqslant_{q n}^{r}$ is a wqo, so that $\leqslant_{T(q n)}^{r}$ is a wqo as well. Undecidability of $\leqslant_{T(q n)}^{r}$ follows from the undecidability of the trace inclusion problem for nondeterministic OCNs [Hofman et al. 2013, Theorem 20] since given the OCNs $O_{1}=\left(Q_{1}, \Sigma, \delta_{1}\right)$ and $O_{2}=\left(Q_{2}, \Sigma, \delta_{2}\right)$, we can define the union OCN $O_{3} \stackrel{\text { def }}{=}\left(Q_{1} \cup Q_{2} \cup\{q\}, \Sigma, \delta_{3}\right)$ where $\delta_{3}$ maps $(q, a, 0)$ to $q_{1} \in Q_{1},(q, b, 0)$ to $q_{2} \in Q_{2}$ and behaves like $\delta_{1}$ or $\delta_{2}$ elsewhere. Then, it turns out that

$$
a \leqslant_{T_{3}(q n)}^{r} b \Leftrightarrow a^{-1} T_{3}\left(q_{1} n\right) \subseteq b^{-1} T_{3}\left(q_{2} n\right) \Leftrightarrow T_{1}\left(q_{1} n\right) \subseteq T_{2}\left(q_{2} n\right) .
$$

Therefore, deciding the right Nerode's quasiorder $\leqslant_{T_{3}(q n)}^{r}$ is as hard as deciding $T_{1}\left(q_{1} n\right) \subseteq T_{2}\left(q_{2} n\right)$.

It is worth to remark that, by Lemma 4.3.5 (a), the left and right Nerode's quasiorders $\leqslant_{T(q n)}^{\ell}$ and $\leqslant_{T(q n)}^{r}$ are $T(q n)$-consistent. However, the left Nerode's quasiorder does not need to be a wqo, otherwise $T(q n)$ would be regular.

We conclude this section by conjecturing that our framework could be instantiated for extending Corollary 4.3.12 to traces of Petri Nets, a result which is already known to be true [Jancar et al. 1999]. 


\subsection{A Novel Perspective on the Antichain Algorithm}

Let $\mathcal{N}_{1}=\left\langle Q_{1}, \delta_{1}, I_{1}, F_{1}, \Sigma\right\rangle$ and $\mathcal{N}_{2}=\left\langle Q_{2}, \delta_{2}, I_{2}, F_{2}, \Sigma\right\rangle$ be two NFAs and consider the state-based left $\mathcal{L}\left(\mathcal{N}_{2}\right)$-consistent wqo $\leqslant_{N_{2}}^{\ell}$ defined by Equivalence (4.12). Theorem 4.3.3 shows that Algorithm FAINcW decides the language inclusion $\mathcal{L}\left(\mathcal{N}_{1}\right) \subseteq \mathcal{L}\left(\mathcal{N}_{2}\right)$ by manipulating finite sets of words.

Since $u \leqslant_{\mathcal{N}_{2}}^{\ell} v \Leftrightarrow \operatorname{pre}_{u}^{\mathcal{N}_{2}}\left(F_{2}\right) \subseteq \operatorname{pre}_{v}^{\mathcal{N}_{2}}\left(F_{2}\right)$, we could equivalently consider the set of states $\operatorname{pre}_{u}^{\mathcal{N}_{2}}\left(F_{2}\right) \in \wp\left(Q_{2}\right)$ rather than a word $u \in \Sigma^{*}$. This observation suggests the design of an algorithm analogous to FAINcW but computing on the poset $\left\langle\mathrm{AC}_{\left\langle\wp\left(Q_{2}\right), \subseteq\right\rangle}, \sqsubseteq\right\rangle$ of antichains of sets of states of the complete lattice $\left\langle\wp\left(Q_{2}\right), \subseteq\right\rangle$.

In order to do this, the poset $\left\langle\mathrm{AC}_{\left\langle\wp\left(Q_{2}\right), \subseteq\right\rangle}\right.$, $\rangle$ is viewed as an abstraction of the poset $\left\langle\wp\left(\Sigma^{*}\right), \subseteq\right\rangle$ by using the abstraction and concretization functions $\alpha: \wp\left(\Sigma^{*}\right) \rightarrow \mathrm{AC}_{\left\langle\wp\left(Q_{2}\right), \subseteq\right\rangle}$ and $\gamma: \mathrm{AC}_{\left\langle\wp\left(Q_{2}\right), \subseteq\right\rangle} \rightarrow \wp\left(\Sigma^{*}\right)$ and using the abstract function $\operatorname{Pre}_{\mathcal{N}_{1}}^{\mathcal{N}_{2}}:\left(\mathrm{AC}_{\left\langle\wp\left(Q_{2}\right), \subseteq\right\rangle}\right)^{\left|Q_{1}\right|} \rightarrow\left(\mathrm{AC}_{\left\langle\wp\left(Q_{2}\right), \subseteq\right\rangle}\right)^{\left|Q_{1}\right|}$ defined as follows:

$$
\begin{aligned}
& \alpha(X) \stackrel{\text { def }}{=}\left\lfloor\left\{\operatorname{pre}_{u}^{\mathcal{N}_{2}}\left(F_{2}\right) \in \wp\left(Q_{2}\right) \mid u \in X\right\}\right\rfloor, \\
& \gamma(Y) \stackrel{\text { def }}{=}\left\{v \in \Sigma^{*} \mid \exists u \in \Sigma^{*}, \operatorname{pre}_{u}^{\mathcal{N}_{2}}\left(F_{2}\right) \in Y \wedge \operatorname{pre}_{u}^{\mathcal{N}_{2}}\left(F_{2}\right) \subseteq \operatorname{pre}_{v}^{\mathcal{N}_{2}}\left(F_{2}\right)\right\}, \\
& \operatorname{Pre}_{\mathcal{N}_{1}}^{\mathcal{N}_{2}}\left(\left\langle X_{q}\right\rangle_{q \in Q_{1}}\right) \stackrel{\text { def }}{=}\left\langle\left\lfloor\left\{\operatorname{pre}_{a}^{\mathcal{N}_{2}}(S) \in \wp\left(Q_{2}\right) \mid \exists a \in \Sigma, q^{\prime} \in Q_{1},\right.\right.\right. \\
& \left.\left.\left.\qquad q^{\prime} \in \delta_{1}(q, a) \wedge S \in X_{q^{\prime}}\right\}\right\rfloor\right\rangle_{q \in Q_{1}} .
\end{aligned}
$$

Observe that the functions $\alpha$ and $\operatorname{Pre}_{\mathcal{N}_{1}}^{\mathcal{N}_{2}}$ are well-defined because minors are antichains.

LEMMA 4.4.1. The following properties hold:
(a) $\left\langle\wp\left(\Sigma^{*}\right), \subseteq\right\rangle \underset{\alpha}{\stackrel{\gamma}{\leftrightarrows}}\left\langle\mathrm{AC}_{\left\langle\wp\left(Q_{2}\right), \subseteq\right\rangle}\right.$, $\left.\rangle\right\rangle$ is a $G C$.
(b) $\gamma \circ \alpha=\rho_{\leqslant_{N_{2}}^{\ell}}$.
(c) For all $\vec{X} \in \alpha\left(\wp\left(\Sigma^{*}\right)\right)^{\left|Q_{1}\right|}, \operatorname{Pre}_{\mathcal{N}_{1}}^{\mathcal{N}_{2}}(\vec{X})=\alpha \circ \operatorname{Pre}_{\mathcal{N}_{1}} \circ \gamma(\vec{X})$.

\section{Proof.}

(a) Let us first observe that $\alpha$ and $\gamma$ are well-defined. First, $\alpha(X)$ is an antichain of $\left\langle\wp\left(Q_{2}\right), \subseteq\right\rangle$ since it is a minor for the well-quasiorder $\subseteq$ and, therefore, it is finite. On the other hand, $\gamma(Y)$ is clearly an element of $\left\langle\wp\left(\Sigma^{*}\right), \subseteq\right\rangle$ by definition.

Then, for all $X \in \wp\left(\Sigma^{*}\right)$ and $Y \in \mathrm{AC}_{\left\langle\wp\left(Q_{2}\right), \subseteq\right\rangle}$, it turns out that:

$$
\alpha(X) \sqsubseteq Y \Leftrightarrow \quad[\text { By definition of } \sqsubseteq]
$$


$\forall z \in \alpha(X), \exists y \in Y, y \subseteq z \Leftrightarrow \quad$ [By definition of $\alpha$ and L·J]

$\forall v \in X, \exists y \in Y, y \subseteq \operatorname{pre}_{v}^{\mathcal{N}_{2}}\left(F_{2}\right) \Leftrightarrow \quad$ [By definition of $\gamma$ ]

$\forall v \in X, v \in \gamma(Y) \Leftrightarrow \quad[$ By definition of $\subseteq$ ]

$$
X \subseteq \gamma(Y) .
$$

(b) For all $X \in \wp\left(\Sigma^{*}\right)$ we have that

$$
\begin{array}{rlrl}
\gamma(\alpha(X))= & {[\text { By definition of } \alpha, \gamma]} \\
\left\{v \in \Sigma^{*} \mid \exists u \in \Sigma^{*}, \operatorname{pre}_{u}^{\mathcal{N}_{2}}\left(F_{2}\right) \in\left\lfloor\left\{\operatorname{pre}_{w}^{\mathcal{N}_{2}}\left(F_{2}\right) \mid w\right.\right.\right. & \left.\in X\}\rfloor \wedge \operatorname{pre}_{u}^{\mathcal{N}_{2}}\left(F_{2}\right) \subseteq \operatorname{pre}_{v}^{\mathcal{N}_{2}}\left(F_{2}\right)\right\} \\
= & {[\text { By definition of minor] }} \\
\left\{v \in \Sigma^{*} \mid \exists u \in X, \operatorname{pre}_{u}^{\mathcal{N}_{2}}\left(F_{2}\right) \subseteq \operatorname{pre}_{v}^{\mathcal{N}_{2}}\left(F_{2}\right)\right\}= & {\left[\text { By definition of } \leqslant_{\mathcal{N}_{2}}^{\ell}\right]} \\
\left\{v \in \Sigma^{*} \mid \exists u \in X, u \leqslant_{\mathcal{N}_{2}}^{\ell} v\right\}= & {\left[\text { By definition of } \rho_{\leqslant_{N_{2}}^{\ell}}\right]} \\
\rho_{\leqslant_{N_{2}}^{\ell}}(X) & &
\end{array}
$$

(c) For all $\vec{X} \in \alpha\left(\wp\left(\Sigma^{*}\right)\right)^{\left|Q_{1}\right|}$ we have that

$$
\begin{aligned}
& \alpha\left(\operatorname{Pre}_{\mathcal{N}_{1}}(\gamma(\vec{X}))\right)= \\
& \text { [By definition of } \operatorname{Pre}_{\mathcal{N}_{1}} \text { ] } \\
& \left\langle\alpha\left(\bigcup_{a \in \Sigma, q \rightarrow N_{1} q^{\prime}} \operatorname{a\gamma }\left(\vec{X}_{q^{\prime}}\right)\right)\right\rangle_{q \in Q_{1}}= \\
& \text { [By definition of } \alpha \text { ] } \\
& \left\langle\left\lfloor\left\{\operatorname{pre}_{u}^{\mathcal{N}_{2}}\left(F_{2}\right) \mid u \in \bigcup_{a \in \Sigma, q{\stackrel{a}{N_{1}}}^{\prime}} \operatorname{ar}\left(\vec{X}_{q^{\prime}}\right)\right\rfloor\right\rangle_{q \in Q_{1}}=\right. \\
& {\left[\text { By pre }{ }_{a v}^{\mathcal{N}_{2}}=\operatorname{pre}_{a}^{\mathcal{N}_{2} \circ \operatorname{pre}_{v}^{\mathcal{N}_{2}}}\right. \text { ] }} \\
& \left\langle\left\lfloor\left\{\operatorname{pre}_{a}^{\mathcal{N}_{2}}\left(\left\{\operatorname{pre}_{u}^{\mathcal{N}_{2}}\left(F_{2}\right) \mid u \in \bigcup_{q \rightarrow{ }_{N_{1}}{ }^{\prime}} \gamma\left(\vec{X}_{q^{\prime}}\right)\right\}\right) \mid a \in \Sigma\right\}\right\rfloor\right\rangle_{q \in Q_{1}}= \\
& \left\langle\left\lfloor\left\{\operatorname{pre}_{a}^{\mathcal{N}_{2}}(S) \mid a \in \Sigma, q \stackrel{a}{\rightarrow} \mathcal{N}_{1} q^{\prime}, S \in\left\{\operatorname{pre}_{u}^{\mathcal{N}_{2}}\left(F_{2}\right) \mid u \in \gamma\left(\vec{X}_{q^{\prime}}\right)\right\}\right\}\right\rfloor\right\rangle_{q \in Q_{1}}= \\
& {\left[\text { By }\left\lfloor\operatorname{pre}_{a}^{\mathcal{N}_{2}}(X)\right\rfloor=\left\lfloor\operatorname{pre}_{a}^{\mathcal{N}_{2}}(\lfloor X\rfloor)\right\rfloor\right]} \\
& \left.\left\langle\left\lfloor\operatorname{pre}_{a}^{\mathcal{N}_{2}}(S) \mid a \in \Sigma, q \stackrel{a}{\rightarrow} \mathcal{N}_{1} q^{\prime}, S \in\left\lfloor\left\{\operatorname{pre}_{u}^{\mathcal{N}_{2}}\left(F_{2}\right) \mid u \in \gamma\left(\vec{X}_{q^{\prime}}\right)\right\}\right\rfloor\right\}\right\rfloor\right\rangle_{q \in Q_{1}}= \\
& \left.\left.\left\langle\operatorname{Lpre}_{a}^{\mathcal{N}_{2}}(S)\right| a \in \Sigma, q \stackrel{a}{\rightarrow} \mathcal{N}_{1} q^{\prime}, S \in \alpha\left(\gamma\left(\vec{X}_{q^{\prime}}\right)\right)\right\rfloor\right\rangle_{q \in Q_{1}}= \\
& \text { [Since } \vec{X} \in \alpha, \alpha\left(\gamma\left(\vec{X}_{q^{\prime}}\right)\right)=\vec{X}_{q^{\prime}} \text { ] }
\end{aligned}
$$




$$
\begin{gathered}
\left\langle\left\lfloor\left\{\operatorname{pre}_{a}^{\mathcal{N}_{2}}(S) \mid a \in \sum, q \stackrel{a}{\rightarrow} \mathcal{N}_{1} q^{\prime}, S \in \vec{X}_{q^{\prime}}\right\}\right\rfloor\right\rangle_{q \in Q_{1}}= \\
{\left[\text { By definition of Pre } \operatorname{Pr}_{\mathcal{N}_{1}}^{\mathcal{N}_{2}}\right]} \\
\operatorname{Pre}_{\mathcal{N}_{1}}^{\mathcal{N}_{2}}(\vec{X})
\end{gathered}
$$

It follows from Lemma 4.4.1 that the GC $\left\langle\wp\left(\Sigma^{*}\right), \subseteq\right\rangle \stackrel{\gamma}{\stackrel{\alpha}{\leftrightarrows}}\left\langle\mathrm{AC}_{\left\langle\wp\left(Q_{2}\right), \subseteq\right\rangle}, \sqsubseteq\right\rangle$ and the abstract function Pre ${ }_{\mathcal{N}_{1}}^{\mathcal{N}_{2}}$ satisfy the hypotheses (i)-(iv) of Theorem 4.2.11. Thus, in order to obtain an algorithm for deciding $\mathcal{L}\left(\mathcal{N}_{1}\right) \subseteq \mathcal{L}\left(\mathcal{N}_{2}\right)$ it remains to show that requirement (v) of Theorem 4.2.11 holds, i.e. there is an algorithm to decide whether $\vec{Y} \sqsubseteq \alpha\left(\overrightarrow{\boldsymbol{L}_{2} I_{2}}\right)$ for every $\vec{Y} \in \alpha\left(\wp\left(\Sigma^{*}\right)\right)^{\left|Q_{1}\right|}$. In order to do that, we first provide some intuitions on how the resulting algorithm works.

First, observe that the Kleene iterates of the function $\lambda \vec{X} \cdot \alpha\left(\overrightarrow{\boldsymbol{\epsilon}}^{F_{1}}\right) \sqcup \operatorname{Pre}_{\mathcal{N}_{1}}^{\mathcal{N}_{2}}(\overrightarrow{\boldsymbol{X}})$ of Theorem 4.2.11 are vectors of antichains in $\left\langle\mathrm{AC}_{\left\langle\wp\left(Q_{2}\right), \subseteq\right\rangle}\right.$, $\rangle$, where each component is indexed by some $q \in Q_{1}$ and represents (through its minor set) a set of sets of states that are predecessors of $F_{2}$ in $\mathcal{N}_{2}$ by a word $u$ generated by $\mathcal{N}_{1}$ from that state $q$, i.e. $\operatorname{pre}_{u}^{\mathcal{N}_{2}}\left(F_{2}\right)$ with $u \in W_{q, F_{1}}^{\mathcal{N}_{1}}$. Since $\epsilon \in W_{q, F_{1}}^{\mathcal{N}_{1}}$ for all $q \in F_{1}$ and pre $_{\epsilon}^{\mathcal{N}_{2}}\left(F_{2}\right)=F_{2}$ the iterations of the procedure KLEENE begin with the initial vector $\alpha\left(\overrightarrow{\boldsymbol{\epsilon}}^{F_{1}}\right)=\left\langle\psi_{\varnothing}^{F_{2}}\left(q \in F_{1}\right)\right\rangle_{q \in Q_{1}}$.

On the other hand, note that by taking the minor of each vector component, we are considering smaller sets which still preserve the relation $\sqsubseteq$ since

$$
A \sqsubseteq B \Leftrightarrow\lfloor A\rfloor \sqsubseteq B \Leftrightarrow A \sqsubseteq\lfloor B\rfloor \Leftrightarrow\lfloor A\rfloor \sqsubseteq\lfloor B\rfloor .
$$

Let $\left\langle Y_{q}\right\rangle_{q \in Q_{1}}$ be the fixpoint computed by the KLEENE procedure. It turns out that, for each component $q \in Q_{1}, Y_{q}=\left\lfloor\left\{\operatorname{pre}_{u}^{\mathcal{N}_{2}}\left(F_{2}\right) \mid u \in W_{q, F_{1}}^{\mathcal{N}_{1}}\right\}\right\rfloor$ holds. Whenever the inclusion $\mathcal{L}\left(\mathcal{N}_{1}\right) \subseteq \mathcal{L}\left(\mathcal{N}_{2}\right)$ holds, all the sets of states in $Y_{q}$ for some initial state $q \in I_{1}$ are predecessors of $F_{2}$ in $\mathcal{N}_{2}$ by words in $\mathcal{L}\left(\mathcal{N}_{2}\right)$, so that they all contain at least one initial state in $I_{2}$. As a result, we obtain Algorithm FAINCS, that is, a "state-based" inclusion algorithm for deciding $\mathcal{L}\left(\mathcal{N}_{1}\right) \subseteq \mathcal{L}\left(\mathcal{N}_{2}\right)$.

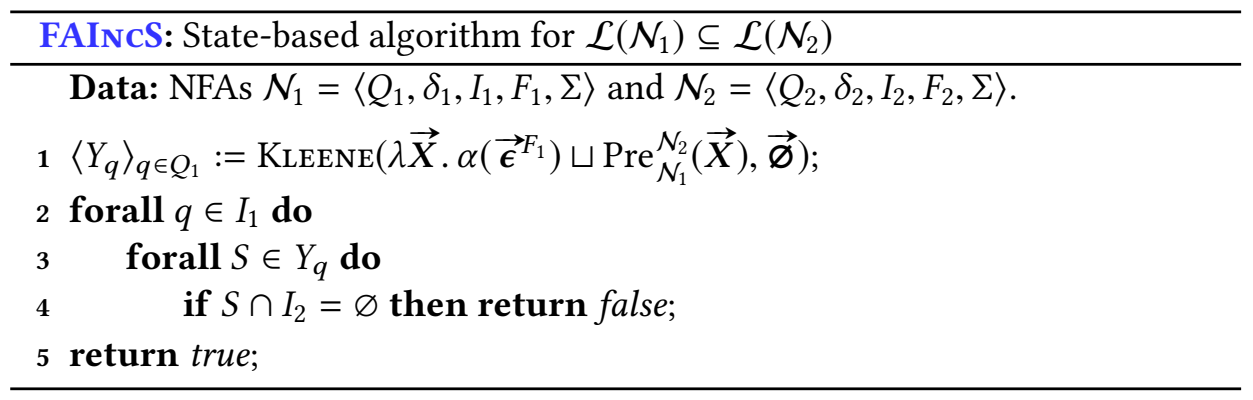


THEOREM 4.4.2. Let $\mathcal{N}_{1}, \mathcal{N}_{2}$ be NFAs. The algorithm FAINCS decides the inclusion $\mathcal{L}\left(\mathcal{N}_{1}\right) \subseteq \mathcal{L}\left(\mathcal{N}_{2}\right)$.

Proof. We show that all the conditions (i)-(v) of Theorem 4.2.11 are satisfied for the abstract domain $\left\langle D, \leqslant_{D}\right\rangle=\left\langle\mathrm{AC}_{\left\langle\wp\left(Q_{2}\right), \subseteq\right\rangle}\right.$, $\left.\sqsubseteq\right\rangle$ as defined by the Galois Connection of Lemma 4.4.1 (a).

(a) Since, by Lemma 4.4.1 (b), $\rho_{\leqslant_{N_{2}}^{\ell}}(X)=\gamma(\alpha(X))$ it follows from Lemmas 4.3.2 and 4.3.7 that $\gamma\left(\alpha\left(L_{2}\right)\right)=L_{2}$. Moreover, for all $a \in \Sigma, X \in \wp\left(\Sigma^{*}\right)$ we have that:

$$
\begin{aligned}
\gamma \alpha(a X)= & {[\text { In GCs } \gamma=\gamma \alpha \gamma] } \\
\gamma \alpha \gamma \alpha(a X)= & {\left[\text { By Lemma 4.3.2 (b) with } \rho_{\leqslant_{N_{2}}^{\ell}}=\gamma \alpha\right] } \\
\gamma \alpha \gamma \alpha(\operatorname{a\gamma } \alpha(X))= & {[\text { In GCs } \gamma=\gamma \alpha \gamma] } \\
\gamma \alpha(\operatorname{ar} \alpha(X)) . &
\end{aligned}
$$

(b) $\left(\mathrm{AC}_{\left\langle\wp\left(Q_{2}\right), \subseteq\right\rangle}\right.$, ᄃ) is effective because $Q_{2}$ is finite.

(c) By Lemma 4.4.1 (c) we have that $\alpha\left(\operatorname{Pre}_{\mathcal{N}_{1}}(\gamma(\vec{X}))\right)=\operatorname{Pre}_{\mathcal{N}_{1}}^{\mathcal{N}_{2}}(\vec{X})$ for all vectors $\vec{X} \in \alpha\left(\wp\left(\Sigma^{*}\right)\right)^{\left|Q_{1}\right|}$.

(d) $\alpha(\{\epsilon\})=\left\{F_{2}\right\}$ and $\alpha(\varnothing)=\varnothing$, hence $\left\lfloor\alpha\left(\overrightarrow{\boldsymbol{\epsilon}}^{F_{1}}\right)\right\rfloor$ is trivial to compute.

(e) Since $\alpha\left({\overrightarrow{L_{2}}}^{I_{1}}\right)=\left\langle\alpha\left(\psi_{\Sigma^{*}}^{L_{2}}\left(q \in I_{1}\right)\right)\right\rangle_{q \in Q_{1}}$, for all $\vec{Y} \in \alpha\left(\wp\left(\Sigma^{*}\right)\right)^{\left|Q_{1}\right|}$ the relation $\vec{Y} \sqsubseteq \alpha\left(\vec{L}_{2}^{I_{1}}\right)$ trivially holds for all components $q \notin I_{1}$. For the components $q \in I_{1}$, it suffices to show that $Y_{q} \sqsubseteq \alpha\left(L_{2}\right) \Leftrightarrow \forall S \in Y_{q}, S \cap I_{2} \neq \varnothing$, which is the check performed by lines $2-5$ of algorithm FAINcS.

$$
\begin{aligned}
& Y_{q} \sqsubseteq \alpha\left(L_{2}\right) \Leftrightarrow\left[\text { Because } Y_{q}=\alpha(U) \text { for some } U \in \wp\left(\Sigma^{*}\right)\right] \\
& \alpha(U) \sqsubseteq \alpha\left(L_{2}\right) \Leftrightarrow[\text { By GC] } \\
& U \subseteq \gamma\left(\alpha\left(L_{2}\right)\right) \Leftrightarrow\left[\text { By L. 4.3.2, 4.3.7 and 4.4.1, } \gamma\left(\alpha\left(L_{2}\right)\right)=L_{2}\right] \\
& U \subseteq L_{2} \Leftrightarrow\left[\text { By definition of } \operatorname{pre}_{u}^{\mathcal{N}_{2}}\right] \\
&\left.\forall u \in U, \operatorname{pre}_{u}^{\mathcal{N}_{2}}\left(F_{2}\right) \cap I_{2} \neq \varnothing \Leftrightarrow\left[\text { Since } Y_{q}=\alpha(U)=\left\lfloor\operatorname{pre}_{u}^{\mathcal{N}_{2}}\left(F_{2}\right) \mid u \in U\right\}\right\rfloor\right] \\
& \forall S \in Y_{q}, S \cap I_{2} \neq \varnothing .
\end{aligned}
$$

Thus, by Theorem 4.2.11, Algorithm FAIncS decides $\mathcal{L}\left(\mathcal{N}_{1}\right) \subseteq \mathcal{L}\left(\mathcal{N}_{2}\right)$.

\subsubsection{Relationship to the Antichains Algorithm}

Wulf et al. [2006] introduced two so-called antichains algorithms, denoted forward and backward, for deciding the universality of the language accepted by an 
NFA, i.e. whether the language is $\Sigma^{*}$ or not. Then, they extended the backward algorithm to decide the inclusion between the languages accepted by two NFAs.

In what follows we show that Algorithm FAINCS is equivalent to the corresponding extension of the forward algorithm and, therefore, dual to the backward antichains algorithm for language inclusion by Wulf et al. [2006][Theorem 6].

To do that, we first define the poset of antichains in which the forward antichains algorithm computes its fixpoint. Then, we give a formal definition of the forward antichains algorithm for deciding language inclusion and show that this algorithm coincides with FAINCS when applied to the reverse automata. Since language inclusion between the languages generated by two NFAs holds iff inclusion holds between the languages generated by their reverse NFAs, we conclude that the algorithm FAINCS is equivalent to the forward antichains algorithm.

Finally, we show how the different variants of the antichains algorithm, including the original backward antichains algorithm [Wulf et al. 2006][Theorem 6], can be derived within our framework by considering the adequate quasiorders.

\section{Forward Antichains Algorithm}

Let $\mathcal{N}_{1}=\left\langle Q_{1}, \Sigma, \delta_{1}, I_{1}, F_{1}\right\rangle$ and $\mathcal{N}_{2}=\left\langle Q_{2}, \Sigma, \delta_{2}, I_{2}, F_{2}\right\rangle$ be two NFAs and consider the language inclusion problem $\mathcal{L}\left(\mathcal{N}_{1}\right) \subseteq \mathcal{L}\left(\mathcal{N}_{2}\right)$. Let us consider the following poset of antichains $\left\langle\mathrm{AC}_{\left\langle\wp\left(Q_{2}\right), \subseteq\right\rangle}, \widetilde{\Xi}\right\rangle$ where

$$
X \widetilde{\subseteq} Y \stackrel{\text { def }}{\Leftrightarrow} \forall y \in Y, \exists x \in X, x \subseteq y
$$

and notice that $\widetilde{\Xi}$ coincides with the reverse relation $\complement^{-1}$. As observed by Wulf et al. [2006, Lemma 1], it turns out that $\left\langle\mathrm{AC}_{\left\langle\wp\left(Q_{2}\right), \subseteq\right\rangle}, \widetilde{\Xi}, \widetilde{\sqcup}, \widetilde{\Pi},\{\varnothing\}, \varnothing\right\rangle$ is a finite lattice, where $\widetilde{\square}$ and $\widetilde{\Pi}$ denote, resp., lub and glb, and $\{\varnothing\}$ and $\varnothing$ are, resp., the least and greatest elements. This lattice $\left\langle\mathrm{AC}_{\left\langle\wp\left(Q_{2}\right), \subseteq\right\rangle}, \widetilde{\Xi}\right\rangle$ is the domain in which the forward antichains algorithm computes on for deciding universality [Wulf et al. 2006, Theorem 3]. The following result extends this forward algorithm in order to decide language inclusion.

TheORem 4.4.3 ([Wulf et al. 2006, Theorems 3 and 6]). Let

$$
\overrightarrow{\mathcal{F P}} \stackrel{\text { def }}{=} \widetilde{\sqcup}\left\{\vec{X} \in\left(\operatorname{AC}_{\left\langle\wp\left(Q_{2}\right), \subseteq\right\rangle}\right)^{\left|Q_{1}\right|} \mid \vec{X}=\operatorname{Post}_{\mathcal{N}_{1}}^{\mathcal{N}_{2}}(\vec{X}) \tilde{\Pi}\left\langle\psi_{\varnothing}^{\left\{I_{2}\right\}}\left(q \in I_{1}\right)\right\rangle_{q \in Q_{1}}\right\}
$$

where

$$
\begin{aligned}
& \operatorname{Post}_{\mathcal{N}_{1}}^{\mathcal{N}_{2}}\left(\left\langle X_{q}\right\rangle_{q \in Q_{1}}\right) \stackrel{\text { def }}{=}\left\langle L \text { post }_{a}^{\mathcal{N}_{2}}(x) \in \wp\left(Q_{2}\right)\right| \exists a \in \Sigma, q^{\prime} \in Q_{1}, \\
& \\
&\left.\left.\left.q \in \delta_{1}\left(q^{\prime}, a\right) \wedge x \in X_{q^{\prime}}\right\}\right\rfloor\right\rangle_{q \in Q_{1}} .
\end{aligned}
$$

Then, $\mathcal{L}\left(\mathcal{N}_{1}\right) \nsubseteq \mathcal{L}\left(\mathcal{N}_{2}\right)$ if and only if there exists $q \in F_{1}$ such that $\overrightarrow{\mathcal{F}}_{q} \widetilde{\Xi}\left\{F_{2}^{c}\right\}$. 
Proof. Let us first introduce some notation necessary to describe the forward antichains algorithm by Wulf et al. [2006] for deciding $\mathcal{L}\left(\mathcal{N}_{1}\right) \subseteq \mathcal{L}\left(\mathcal{N}_{2}\right)$. Let us consider the poset $\left\langle Q_{1} \times \wp\left(Q_{2}\right), \subseteq_{\times}\right\rangle$where

$$
\left(q_{1}, x_{1}\right) \subseteq_{\times}\left(q_{2}, x_{2}\right) \stackrel{\text { def }}{\Leftrightarrow} q_{1}=q_{2} \wedge x_{1} \subseteq x_{2} .
$$

Then, let $\left\langle\mathrm{AC}_{\left\langle Q_{1} \times \wp\left(Q_{2}\right), \subseteq_{x}\right\rangle}, \widetilde{\Xi}_{x}, \widetilde{\sqcup}_{\times}, \widetilde{\Pi}_{x}\right\rangle$ be the lattice of antichains over the poset $\left\langle Q_{1} \times \wp\left(Q_{2}\right), \subseteq_{\times}\right\rangle$where:

$$
\begin{aligned}
X \widetilde{\Xi}_{X} Y & \stackrel{\text { def }}{\Leftrightarrow} \forall(q, y) \in Y, \exists(q, x) \in X, x \subseteq y \\
\min _{\times}(X) & \stackrel{\text { def }}{=}\left\{(q, x) \in X \mid \forall\left(q^{\prime}, x^{\prime}\right) \in X, q=q^{\prime} \Rightarrow x^{\prime} \nsubseteq x\right\} \\
X \widetilde{\sqcup}_{X} Y & \stackrel{\text { def }}{=} \min _{\times}(\{(q, x \cup y) \mid(q, x) \in X,(q, y) \in Y\}) \\
X \widetilde{\Pi}_{X} Y & \stackrel{\text { def }}{=} \min _{\times}(\{(q, z) \mid(q, z) \in X \cup Y\}) .
\end{aligned}
$$

Also, let Post : $\mathrm{AC}_{\left\langle Q_{1} \times \wp\left(Q_{2}\right), \subseteq_{x}\right\rangle} \rightarrow \mathrm{AC}_{\left\langle Q_{1} \times \wp\left(Q_{2}\right), \subseteq_{x}\right\rangle}$ be defined as follows:

$$
\begin{aligned}
\operatorname{Post}(X) \stackrel{\text { def }}{=} \min _{\times}\left(\left\{\left(q, \operatorname{post}_{a}^{\mathcal{N}_{2}}(x)\right) \in Q_{1} \times \wp\left(Q_{2}\right) \mid \exists a \in \Sigma, q \in Q_{1},\right.\right. \\
\\
\left.\left.\quad\left(q^{\prime}, x\right) \in X, q^{\prime} \stackrel{a}{\rightarrow} \mathcal{N}_{1} q\right\}\right) .
\end{aligned}
$$

Then, it turns out that the dual of the backward antichains algorithm of Wulf et al. [2006, Theorem 6] states that $\mathcal{L}\left(\mathcal{N}_{1}\right) \nsubseteq \mathcal{L}\left(\mathcal{N}_{2}\right)$ iff there exists $q \in F_{1}$ such that $\mathcal{F} \mathcal{P} \widetilde{\Xi}_{\times}\left\{\left(q, F_{2}^{c}\right)\right\}$ where

$$
\mathcal{F P}=\widetilde{\bigsqcup}_{\times}\left\{X \in \mathrm{AC}_{\left\langle Q_{1} \times \varphi_{(}\left(Q_{2}\right), \subseteq_{\times}\right\rangle} \mid X=\operatorname{Post}(X) \widetilde{\Pi}_{\times}\left(I_{1} \times\left\{I_{2}\right\}\right)\right\} .
$$

We observe that for every $X \in \operatorname{AC}_{\left\langle Q_{1} \times \wp\left(Q_{2}\right), \subseteq_{X}\right\rangle}$, a pair $(q, x) \in Q_{1} \times \wp\left(Q_{2}\right)$ such that $(q, x) \in X$ is used by Wulf et al. [2006, Theorem 6] simply as a way to associate states $q$ of $\mathcal{N}_{1}$ with sets $x$ of states of $\mathcal{N}_{2}$. In fact, every antichain $X \in \mathrm{AC}_{\left\langle Q_{1} \times \wp\left(Q_{2}\right), \subseteq_{x}\right\rangle}$ can be equivalently formalized by a vector

$$
\left\langle\left\{x \in \wp\left(Q_{2}\right) \mid(q, x) \in X\right\}\right\rangle_{q \in Q_{1}} \in\left(\operatorname{AC}_{\left\langle\wp\left(Q_{2}\right), \subseteq\right\rangle}\right)^{\left|Q_{1}\right|}
$$

indexed by states $q \in Q_{1}$ and whose components are antichains in $\mathrm{AC}_{\left\langle\wp\left(Q_{2}\right), \subseteq\right\rangle}$.

Correspondingly, we consider the lattice $\left\langle\mathrm{AC}_{\left\langle\wp\left(Q_{2}\right), \subseteq\right\rangle}\right.$, $\left.\widetilde{\Xi}\right\rangle$, where for every pair of elements $X, Y \in \mathrm{AC}_{\left\langle\wp\left(Q_{2}\right), \subseteq\right\rangle}$ we have that

$$
X \widetilde{\subseteq} Y \stackrel{\text { def }}{\Leftrightarrow} \forall y \in Y, \exists x \in X, x \subseteq y
$$




$$
\begin{aligned}
& \min (X) \stackrel{\text { def }}{=}\left\{x \in X \mid \forall x^{\prime} \in X, x^{\prime} \not \subset x\right\} \\
& X \widetilde{\sqcup} Y \stackrel{\text { def }}{=} \min \left(\left\{x \cup y \in \wp\left(Q_{2}\right) \mid x \in X, y \in Y\right\}\right) \\
& X \widetilde{\sqcap} Y \stackrel{\text { def }}{=} \min \left(\left\{z \in \wp\left(Q_{2}\right) \mid z \in X \cup Y\right\}\right) .
\end{aligned}
$$

Then, Post can be replaced by $\operatorname{Post}_{\mathcal{N}_{1}}^{\mathcal{N}_{2}}:\left(\mathrm{AC}_{\left\langle\wp\left(Q_{2}\right), \subseteq\right\rangle}\right)^{\left|Q_{1}\right|} \rightarrow\left(\mathrm{AC}_{\left\langle\wp\left(Q_{2}\right), \subseteq\right\rangle}\right)^{\left|Q_{1}\right|}$, its equivalent formulation on vectors defined as follows:

$$
\begin{aligned}
& \operatorname{Post}_{\mathcal{N}_{1}}^{\mathcal{N}_{2}}\left(\left\langle X_{q}\right\rangle_{q \in Q_{1}}\right) \stackrel{\text { def }}{=}\left\langle\operatorname { m i n } \left(\left\{\operatorname{post}_{a}^{\mathcal{N}_{2}}(x) \in \wp\left(Q_{2}\right) \mid \exists a \in \Sigma, q^{\prime} \in Q_{1},\right.\right.\right. \\
&\left.\left.\left.x \in X_{q^{\prime}}, q^{\prime} \stackrel{a}{\rightarrow} \mathcal{N}_{1} q\right\}\right)\right\rangle_{q \in Q_{1}} .
\end{aligned}
$$

In turn, $\mathcal{F P} \in \mathrm{AC}_{\left\langle Q_{1} \times \wp\left(Q_{2}\right), \subseteq_{\times}\right\rangle}$is replaced by the following vector:

$$
\overrightarrow{\mathcal{F P}} \stackrel{\text { def }}{=} \widetilde{\bigsqcup}\left\{\vec{X} \in\left(\mathrm{AC}_{\left\langle\wp\left(Q_{2}\right), \subseteq\right\rangle}\right)^{\left|Q_{1}\right|} \mid \vec{X}=\operatorname{Post}_{\mathcal{N}_{1}}^{\mathcal{N}_{2}}(\vec{X}) \widetilde{\Pi}\left\langle\psi_{\varnothing}^{\left\{I_{2}\right\}}\left(q \in I_{1}\right)\right\rangle_{q \in Q_{1}}\right\} .
$$

Finally, the check $\exists q \in F_{1}, \mathcal{F P} \widetilde{\Xi}_{\times}\left\{\left(q, F_{2}^{c}\right)\right\}$ becomes $\exists q \in F_{1}, \overrightarrow{\mathcal{F P}}_{q} \widetilde{\subseteq}\left\{F_{2}^{c}\right\}$

Let $\mathcal{N}^{R}$ denote the reverse automaton of $\mathcal{N}$, where arrows are flipped and the initial/final states become final/initial. Note that language inclusion can be decided by considering the reverse automata since

$$
\mathcal{L}\left(\mathcal{N}_{1}\right) \subseteq \mathcal{L}\left(\mathcal{N}_{2}\right) \Leftrightarrow \mathcal{L}\left(\mathcal{N}_{1}^{R}\right) \subseteq \mathcal{L}\left(\mathcal{N}_{2}^{R}\right)
$$

Furthermore, it is straightforward to check that Post ${ }_{\mathcal{N}_{1}}^{\mathcal{N}_{2}}=\operatorname{Pre}_{\mathcal{N}_{1}^{R}}^{\mathcal{N}_{2}^{R}}$. We therefore obtain the following result as a consequence of Theorem 4.4.3.

COROLlary 4.4.4. Let

$$
\overrightarrow{\mathcal{F P}} \stackrel{\text { def }}{=} \widetilde{\square}\left\{\vec{X} \in\left(\mathrm{AC}_{\left\langle\wp\left(Q_{2}\right), \subseteq\right\rangle}\right)^{\left|Q_{1}\right|} \mid \vec{X}=\operatorname{Pre}_{\mathcal{N}_{1}}^{\mathcal{N}_{2}}(\vec{X}) \widetilde{\Pi}\left\langle\psi_{\varnothing}^{\left\{F_{2}\right\}}\left(q \in F_{1}\right)\right\rangle_{q \in Q_{1}}\right\} .
$$

Then, $\mathcal{L}\left(\mathcal{N}_{1}\right) \nsubseteq \mathcal{L}\left(\mathcal{N}_{2}\right)$ iff there exists $q \in I_{1}$ such that $\overrightarrow{\mathcal{F P}}_{q} \widetilde{\Xi}\left\{I_{2}^{c}\right\}$

\section{From the Forward Antichains Algorithm to FAIncS}

Since $\widetilde{\Xi}=\sqsubseteq^{-1}$, we have that $\widetilde{\Pi}=\sqcup, \widetilde{\sqcup}=\sqcap$ and the greatest element $\varnothing$ for $\widetilde{\Xi}$ is the least element for $\sqsubseteq$. Moreover, by (4.17), $\alpha\left(\overrightarrow{\boldsymbol{\epsilon}}^{F_{1}}\right)=\left\langle\psi_{\varnothing}^{\left\{F_{2}\right\}}\left(q \in F_{1}\right)\right\rangle_{q \in Q_{1}}$. Therefore, we can rewrite the vector $\overrightarrow{\mathcal{F P}}$ of Corollary 4.4 .4 as

$$
\overrightarrow{\mathcal{F P}}=\prod\left\{\overrightarrow{\boldsymbol{X}} \in\left(\mathrm{AC}_{\left\langle\wp\left(Q_{2}\right), \subseteq\right\rangle}\right)^{\left|Q_{1}\right|} \mid \overrightarrow{\boldsymbol{X}}=\operatorname{Pre}_{\mathcal{N}_{1}}^{\mathcal{N}_{2}}(\vec{X}) \sqcup \alpha\left(\overrightarrow{\boldsymbol{\epsilon}}^{F_{1}}\right)\right\}
$$

which is precisely the lfp in $\left\langle\left(\mathrm{AC}_{\left\langle\wp\left(Q_{2}\right), \subseteq\right\rangle}\right)^{\left|Q_{1}\right|}, \sqsubseteq\right\rangle$ of $\operatorname{Pre}_{\mathcal{N}_{1}}^{\mathcal{N}_{2}}$ above $\alpha\left(\overrightarrow{\boldsymbol{\epsilon}}^{F_{1}}\right)$. 
Hence, it turns out that the Kleene iterates of the least fixpoint computation that converge to $\overrightarrow{\mathcal{F P}}$ exactly coincide with the iterates computed by the KLEENE procedure of the state-based algorithm FAINCS. In particular, if $\vec{Y}$ is the output vector of the call to KLEENE at line 1 of FAINCS then $\vec{Y}=\overrightarrow{\mathcal{F P}}$. Furthermore,

$$
\exists q \in I_{1}, \overrightarrow{\mathcal{F P}}_{q} \widetilde{\Xi}\left\{I_{2}^{c}\right\} \Leftrightarrow \exists q \in I_{1}, \exists S \in \overrightarrow{\mathcal{F P}}_{q}, S \cap I_{2}=\varnothing .
$$

Summing up, the $\sqsubseteq$-lfp algorithm FAINcS coincides with the $\widetilde{\Xi}$-gfp antichains algorithm given by Corollary 4.4.4.

\section{Backward Antichains Algorithm}

We can also derive an antichains algorithm for deciding language inclusion fully equivalent to the backward one of Wulf et al. [2006][Theorem 6] by considering

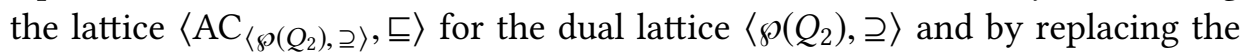
functions $\alpha, \gamma$ and $\operatorname{Pre}_{\mathcal{N}_{1}}^{\mathcal{N}_{2}}$ of Lemma 4.4.1, respectively, with:

$$
\begin{aligned}
& \alpha^{c}(X) \stackrel{\text { def }}{=}\left\lfloor\left\{\operatorname{cpre}_{u}^{\mathcal{N}_{2}}\left(F_{2}^{c}\right) \in \wp\left(Q_{2}\right) \mid u \in X\right\}\right\rfloor, \\
& \gamma^{c}(Y) \stackrel{\text { def }}{=}\left\{u \in \Sigma^{*} \mid \exists y \in Y, y \supseteq \operatorname{cpre}_{u}^{\mathcal{N}_{2}}\left(F_{2}^{c}\right)\right\}, \\
& \operatorname{CPre}_{\mathcal{N}_{1}}^{\mathcal{N}_{2}}\left(\left\langle X_{q}\right\rangle_{q \in Q_{1}}\right) \stackrel{\text { def }}{=}\left\langle\left\lfloor\left\{\operatorname{cpre}_{a}^{\mathcal{N}_{2}}(S) \in \wp\left(Q_{2}\right) \mid \exists a \in \Sigma, q^{\prime} \in Q_{1},\right.\right.\right. \\
& \left.\left.\left.q^{\prime} \in \delta_{1}(q, a) \wedge S \in X_{q^{\prime}}\right\}\right\rfloor\right\rangle_{q \in Q_{1}} .
\end{aligned}
$$

where $\operatorname{cpre}_{u}^{\mathcal{N}_{2}}(S) \stackrel{\text { def }}{=}\left(\operatorname{pre}_{u}^{\mathcal{N}_{2}}\left(S^{c}\right)\right)^{c}$ for $u \in \Sigma^{*}$.

When instantiating Theorem 4.2.11 using these functions, we obtain an lfp

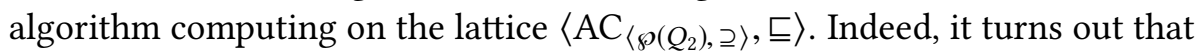

$$
\mathcal{L}\left(\mathcal{N}_{1}\right) \subseteq \mathcal{L}\left(\mathcal{N}_{2}\right) \Leftrightarrow \operatorname{KLEENE}\left(\lambda \vec{X} . \operatorname{CPre}_{\mathcal{N}_{1}}^{\mathcal{N}_{2}}(\vec{X}) \sqcup \alpha^{c}\left(\overrightarrow{\boldsymbol{\epsilon}}^{F_{1}}\right), \vec{\varnothing}\right) \sqsubseteq \alpha^{c}\left({\overrightarrow{\boldsymbol{L}_{2}}}^{I_{1}}\right) .
$$

It is easily seen that this algorithm coincides with the backward antichains algorithm defined by Wulf et al. [2006, Theorem 6] since both compute on the same lattice, $\lfloor X\rfloor$ corresponds to the maximal (w.r.t. set inclusion) elements of $X$, $\alpha^{c}(\{\epsilon\})=\left\{F_{2}^{c}\right\}$ and for all $X \in \alpha^{c}\left(\wp\left(\Sigma^{*}\right)\right), X \sqsubseteq \alpha^{c}\left(L_{2}\right) \Leftrightarrow \forall S \in X, I_{2} \nsubseteq S$.

\section{Variants of the Antichains Algorithm}

We have shown that the two forward/backward antichains algorithms introduced by Wulf et al. [2006] can be systematically derived by instantiating our framework and (possibly) considering the reverse automata. Similarly, we can derive within our framework an algorithm equivalent to the backward antichains algorithm applied to the reverse automata and an algorithm equivalent to the 
forward antichains algorithm (without reverting the automata). Table 4.1 summarizes the relation between our framework and the antichains algorithms given (explicitly or implicitly) by Wulf et al. [2006].

\begin{tabular}{c|c|c} 
& Backward & Forward \\
\hline $\mathcal{L}\left(\mathcal{N}_{1}\right) \subseteq \mathcal{L}\left(\mathcal{N}_{2}\right)$ & $\operatorname{cpre}_{u}^{\mathcal{N}_{2}}\left(F_{2}^{c}\right) \subseteq \operatorname{cpre}_{v}^{\mathcal{N}_{2}}\left(F_{2}^{c}\right)$ & $\operatorname{pre}_{u}^{\mathcal{N}_{2}}\left(I_{2}\right) \subseteq \operatorname{pre}_{v}^{\mathcal{N}_{2}}\left(I_{2}\right)$ \\
$\mathcal{L}\left(\mathcal{N}_{1}^{R}\right) \subseteq \mathcal{L}\left(\mathcal{N}_{2}^{R}\right)$ & $\operatorname{cpost}_{u}^{\mathcal{N}_{2}}\left(I_{2}^{c}\right) \subseteq \operatorname{cpost}_{v}^{\mathcal{N}_{2}}\left(I_{2}^{c}\right)$ & $\operatorname{post}_{u}^{\mathcal{N}_{2}}\left(I_{2}\right) \subseteq \operatorname{post}_{v}^{\mathcal{N}_{2}}\left(I_{2}\right)$
\end{tabular}

Table 4.1: Summary of the quasiorders that should be used within our framework, i.e. using Theorem 4.2.11, to derive the different antichains algorithms that are (explicitly or implicitly) given by Wulf et al. [2006]. Each cell of the form $f(u) \subseteq f(v)$ is the definition of the quasiorder $u \leqslant v \stackrel{\text { def }}{=} f(u) \subseteq f(v)$ that should be used to derive the antichains algorithm given by the column for solving the language inclusion given by the row.

The original antichains algorithms were later improved by Abdulla et al. [2010] and, subsequently, by Bonchi and Pous [2013]. Among their improvements, they showed how to exploit a precomputed binary relation between pairs of states of the input automata such that language inclusion holds for all the pairs in the relation. When that binary relation is a simulation relation, our framework allows to partially match their results by using the quasiorder $\leq_{\mathcal{N}}^{r}$ defined in Section 4.3.3. However, this quasiorder relation $\leq_{\mathcal{N}}^{r}$ does not consider pairs of states $Q_{1} \times Q_{1}$ whereas the aforementioned algorithms do.

\subsection{Inclusion for Context Free Languages}

In Section 4.2 we used the general abstraction scheme presented in Section 4.1 to derive two techniques (Theorems 4.2 .10 and 4.2.11) for defining algorithms for solving language inclusion problems. Then, in Sections 4.3 and 4.4 we applied these techniques on different scenarios and derived algorithms for solving language inclusion problems $L_{1} \subseteq L_{2}$ where $L_{1}$ and $L_{2}$ are regular languages.

In this section, we show that the abstraction scheme from Section 4.1 is general enough to cover language inclusion problems $L_{1} \subseteq L_{2}$ where $L_{1}$ is contextfree. In particular, we replicate the developments from Sections 4.2, 4.3 and 4.4 in order to extend our quasiorder-based framework for deciding the inclusion $L_{1} \subseteq L_{2}$ where $L_{1}$ is a context-free language and $L_{2}$ is regular. 


\subsubsection{Extending the Framework to CFGs}

Similarly to the case of automata, a CFG $\mathcal{G}=(\mathcal{V}, \Sigma, P)$ in CNF induces the following set of equations:

$$
\operatorname{Eqn}(\mathcal{G}) \stackrel{\text { def }}{=}\left\{X_{i}=\bigcup_{X_{i} \rightarrow \beta_{j} \in P} \beta_{j} \mid i \in[0, n]\right\} .
$$

Given a subset of variables $S \subseteq \mathcal{V}$ of a grammar, the set of words generated from some variable in $S$ is defined as

$$
W_{S}^{\mathcal{G}} \stackrel{\text { def }}{=}\left\{w \in \Sigma^{*} \mid \exists X \in S, X \rightarrow^{*} w\right\} .
$$

When $S=\{X\}$ we slightly abuse the notation and write $W_{X}^{\mathcal{G}}$. Also, we drop the superscript $\mathcal{G}$ when the grammar is clear from the context. The language generated by $\mathcal{G}$ is therefore $\mathcal{L}(\mathcal{G})=W_{X_{0}}^{\mathcal{G}}$.

Next, we define the function $\mathrm{Fn}_{\mathcal{G}}: \wp\left(\Sigma^{*}\right)^{|\mathcal{V}|} \rightarrow \wp\left(\Sigma^{*}\right)^{|\mathcal{V}|}$ and the vector $\vec{b} \in \wp\left(\Sigma^{*}\right)^{|\mathcal{V}|}$, which are used to formalize the equations in $\operatorname{Eqn}(\mathcal{G})$, as follows:

$$
\vec{b} \stackrel{\text { def }}{=}\left\langle b_{i}\right\rangle_{i \in[0, n]} \in \wp\left(\Sigma^{*}\right)^{|\mathcal{V}|} \quad \text { with } b_{i} \stackrel{\text { def }}{=}\left\{\beta \mid X_{i} \rightarrow \beta \in P, \beta \in \Sigma \cup\{\epsilon\}\right\},
$$

$\operatorname{Fn}_{\mathcal{G}}(\vec{X}) \stackrel{\text { def }}{=}\left\langle\beta_{1}^{(i)} \cup \ldots \cup \beta_{k_{i}}^{(i)}\right\rangle_{i \in[0, n]} \quad$ with $\beta_{j}^{(i)} \in \mathcal{V}^{2}$ and $X_{i} \rightarrow \beta_{j}^{(i)} \in P$.

Notice that function $\lambda \vec{X} \cdot \vec{b} \cup \mathrm{Fn}_{\mathcal{G}}(\vec{X})$ is a well-defined monotone function in $\wp\left(\Sigma^{*}\right)^{|\mathcal{V}|} \rightarrow \wp\left(\Sigma^{*}\right)^{|\mathcal{V}|}$, which therefore has the least fixpoint

$$
\left\langle Y_{i}\right\rangle_{i \in[0, n]}=\operatorname{lfp}\left(\lambda \vec{X} \cdot \vec{b} \cup \operatorname{Fn}_{\mathcal{G}}(\vec{X})\right)
$$

It is known [Ginsburg and Rice 1962] that the language $\mathcal{L}(\mathcal{G})$ accepted by $\mathcal{G}$ is such that $\mathcal{L}(\mathcal{G})=Y_{0}$.

Example 4.5.1. Consider the following grammar in CNF:

$$
\mathcal{G}=\left\langle\left\{X_{0}, X_{1}\right\},\{a, b\},\left\{X_{0} \rightarrow X_{0} X_{1}\left|X_{1} X_{0}\right| b, X_{1} \rightarrow a\right\}\right\rangle .
$$

The corresponding equation system is

$$
\operatorname{Eqn}(\mathcal{G})=\left\{\begin{array}{l}
X_{0}=X_{0} X_{1} \cup X_{1} X_{0} \cup\{b\} \\
X_{1}=\{a\}
\end{array}\right.
$$

so that

$$
\left(\begin{array}{c}
W_{X_{0}} \\
W_{X_{1}}
\end{array}\right)=\operatorname{lfp}\left(\lambda\left(\begin{array}{c}
X_{0} \\
X_{1}
\end{array}\right) \cdot\left(\begin{array}{c}
X_{0} X_{1} \cup X_{1} X_{0} \cup\{b\} \\
\{a\}
\end{array}\right)\right)=\left(\begin{array}{c}
a^{*} b a^{*} \\
a
\end{array}\right) .
$$

Moreover, we have that $\overrightarrow{\boldsymbol{b}} \in \wp\left(\Sigma^{*}\right)^{2}$ and $\mathrm{Fn}_{\mathcal{G}}: \wp\left(\Sigma^{*}\right)^{2} \rightarrow \wp\left(\Sigma^{*}\right)^{2}$ are given by

$$
\vec{b}=\langle\{b\},\{a\}\rangle \quad \operatorname{Fn}_{\mathcal{G}}\left(\left\langle X_{0}, X_{1}\right\rangle\right)=\left\langle X_{0} X_{1} \cup X_{1} X_{0}, \varnothing\right\rangle
$$


Thus, it follows from Equation (4.18) that

$$
\mathcal{L}(\mathcal{G}) \subseteq L_{2} \Leftrightarrow \operatorname{lfp}\left(\lambda \vec{X} \cdot \vec{b} \cup \operatorname{Fn}_{\mathcal{G}}(\vec{X})\right) \subseteq{\overrightarrow{L_{2}}}^{X_{0}}
$$

where $\overrightarrow{\boldsymbol{L}_{2}} X_{0} \stackrel{\text { def }}{=}\left\langle\psi_{\Sigma^{*}}^{L_{2}}(i=0)\right\rangle_{i \in[0, n]}$.

As we did for the automata case in Section 4.2, we next apply Theorem 4.1.1 in order to derive algorithms for solving the language inclusion problem $\mathcal{L}(\mathcal{G}) \subseteq L_{2}$ by using backward complete abstractions of $\wp\left(\Sigma^{*}\right)$.

THEOREM 4.5.2. Let $\rho \in \operatorname{uco}\left(\wp\left(\Sigma^{*}\right)\right)$ be backward complete for both $\lambda X . X a$ and $\lambda X . a X$, for all $a \in \Sigma$ and let $\mathcal{G}=(\mathcal{V}, \Sigma, P)$ be a $C F G$ in $C N F$. Then $\rho$ is backward complete for $\operatorname{Fn}_{\mathcal{G}}$ and $\lambda \vec{X} \cdot \vec{b} \cup \operatorname{Fn}_{\mathcal{G}}(\vec{X})$.

Proof. Let us first show that backward completeness for left and right concatenation can be extended from letter to words. We give the proof for the concatenation to the left, the case of the concatenation to the right is symmetric. We prove that $\rho(w X)=\rho(w \rho(X))$ for every $w \in \Sigma^{*}$. We proceed by induction on the length of $w$.

The base case is trivial because $\rho$ is idempotent. For the inductive case $|w|>$ 0 let $w=a u$ for some $u \in \Sigma^{*}$ and $a \in \Sigma$, so that:

$$
\begin{aligned}
\rho(a u X)= & {[\text { By backward completeness for } \lambda X . a X] } \\
\rho(a \rho(u X))= & \text { By inductive hypothesis }] \\
\rho(a \rho(u \rho(X)))= & \text { [By backward completeness for } \lambda X . a X] \\
\rho(a u \rho(X)) . &
\end{aligned}
$$

Next we turn to the binary concatenation case, i.e. we prove that $\rho(Y Z)=$ $\rho(\rho(Y) \rho(Z))$ for all $Y, Z \in \wp\left(\Sigma^{*}\right)$ :

$$
\begin{aligned}
& \rho(\rho(Y) \rho(Z))=\text { [By definition of concatenation] } \\
& \rho\left(\bigcup_{u \in \rho(Y)} u \rho(Z)\right)=\text { [By Equation (3.2)] } \\
& \left.\rho\left(\bigcup_{u \in \rho(Y)} \rho(u \rho(Z))\right)=\quad \text { [By backward completeness of } \lambda X . w X\right] \\
& \rho\left(\bigcup_{u \in \rho(Y)} \rho(u Z)\right)=\quad[\text { By Equation (3.2)] } \\
& \rho\left(\cup_{u \in \rho(Y)} u Z\right)=\text { [By definition of concatenation] } \\
& \rho(\rho(Y) Z)=\text { [By definition of concatenation] } \\
& \rho\left(\cup_{v \in Z} \rho(Y) v\right)=\text { [By Equation (3.2)] } \\
& \rho\left(\bigcup_{v \in Z} \rho(\rho(Y) v)\right)=\text { [By backward completeness of } \lambda X . X w \text { ] } \\
& \rho\left(\bigcup_{v \in Z} \rho(Y v)\right)=\text { [By Equation (3.2)] }
\end{aligned}
$$




$$
\begin{array}{r}
\left.\rho\left(\bigcup_{v \in Z} Y v\right)=\quad \text { [By definition of concatenation }\right] \\
\rho(Y Z) .
\end{array}
$$

Then, the proof follows the same lines of the proof of Theorem 4.2.3. Indeed, it follows from the definition of $\mathrm{Fn}_{\mathcal{G}}\left(\left\langle X_{i}\right\rangle_{i \in[0, n]}\right)$ that:

$$
\begin{aligned}
& \left.\rho\left(\bigcup_{j=1}^{k_{i}} \beta_{j}^{(i)}\right)=\quad \text { [By definition of } \beta_{j}^{(i)}\right] \\
& \rho\left(\bigcup_{j=1}^{k_{i}} X_{j}^{(i)} Y_{j}^{(i)}\right)=\quad \text { [By Equation (3.2)] } \\
& \rho\left(\bigcup_{j=1}^{k_{i}} \rho\left(X_{j}^{(i)} Y_{j}^{(i)}\right)\right)=\text { [By backward comp. of } \rho \text { for concatenation] } \\
& \rho\left(\bigcup_{j=1}^{k_{i}} \rho\left(\rho\left(X_{j}^{(i)}\right) \rho\left(Y_{j}^{(i)}\right)\right)\right)=\quad \text { [By Equation (3.2)] } \\
& \rho\left(\bigcup_{j=1}^{k_{i}} \rho\left(X_{j}^{(i)}\right) \rho\left(Y_{j}^{(i)}\right)\right) \text {. }
\end{aligned}
$$

Hence, by a straightforward componentwise application on vectors in $\wp\left(\Sigma^{*}\right)^{|\mathcal{V}|}$, we obtain that $\rho$ is backward complete for $\mathrm{Fn}_{\mathcal{G}}$. Finally, $\rho$ is backward complete for $\lambda \vec{X} .\left(\vec{b} \cup \operatorname{Fn}_{\mathcal{G}}(\vec{X})\right)$, because:

$$
\begin{aligned}
\rho\left(\overrightarrow{\boldsymbol{b}} \cup \mathrm{Fn}_{\mathcal{G}}(\rho(\overrightarrow{\boldsymbol{X}}))\right)= & {[\text { By Equation }(3.2)] } \\
\rho\left(\rho(\overrightarrow{\boldsymbol{b}}) \cup \rho\left(\operatorname{Fn}_{\mathcal{G}}(\rho(\vec{X}))\right)\right)= & {\left[\text { By backward comp. for } \mathrm{Fn}_{\mathcal{G}}\right] } \\
\rho\left(\rho(\overrightarrow{\boldsymbol{b}}) \cup \rho\left(\operatorname{Fn}_{\mathcal{G}}(\vec{X})\right)\right)= & {[\text { By Equation }(3.2)] } \\
\rho\left(\overrightarrow{\boldsymbol{b}} \cup \mathrm{Fn}_{\mathcal{G}}(\overrightarrow{\boldsymbol{X}})\right) . &
\end{aligned}
$$

As a consequence, by backward completeness of $\rho$ for $\lambda \vec{X} .\left(\vec{b} \cup \mathrm{Fn}_{\mathcal{G}}(\vec{X})\right)$, by (4.1) it turns out that:

$$
\rho\left(\operatorname{lfp}\left(\lambda \vec{X} \cdot \vec{b} \cup \operatorname{Fn}_{\mathcal{G}}(\vec{X})\right)\right)=\operatorname{lfp}\left(\lambda \vec{X} \cdot \rho\left(\vec{b} \cup \operatorname{Fn}_{\mathcal{G}}(\vec{X})\right)\right) .
$$

Note that if $\rho$ is backward complete for both left and right concatenation and $\rho\left(L_{2}\right)=L_{2}$ then, as a straightforward consequence of Equivalence (4.19) and Theorems 4.1.1 and 4.5.2, we have that:

$$
\mathcal{L}(\mathcal{G}) \subseteq L_{2} \Leftrightarrow \operatorname{lfp}\left(\lambda \vec{X} \cdot \rho\left(\vec{b} \cup \operatorname{Fn}_{\mathcal{G}}(\vec{X})\right)\right) \subseteq{\overrightarrow{L_{2}}}^{X_{0}}
$$

Next, we present two techniques for solving the language inclusion problem $\mathcal{L}(\mathcal{G}) \subseteq L_{2}$ by relying on Equivalence (4.20). As with the two techniques presented in Section 4.2.3, the first of these techniques allows us to define algorithms for deciding the inclusion by working on finite languages while the second one relies on the use of Galois Connections. 


\subsubsection{Solving the Abstract Inclusion Check using Finite Languages}

The following result, which is an adaptation of Corollary 4.2 .8 for grammars, shows that the fixpoint iteration for $\operatorname{lfp}\left(\rho\left(\overrightarrow{\boldsymbol{b}} \cup \mathrm{Fn}_{\mathcal{G}}(\vec{X})\right)\right)$ can be replicated by iterating on a set of functions $\mathcal{F}$, and then abstracting the result, provided that all functions in $\mathcal{F}$ meet a set of requirements.

LEMMA 4.5.3. Let $\mathcal{G}=\langle\mathcal{V}, \Sigma, P\rangle$ be a $C F G$ in $C N F$, let $\rho \in \mathrm{uco}\left(\Sigma^{*}\right)$ be backward complete for $\lambda X \in \wp\left(\Sigma^{*}\right)$. $a X$ and $\lambda X \in \wp\left(\Sigma^{*}\right)$.Xa for all $a \in \Sigma$ and let $\mathcal{F}$ be a set of functions such that every $f \in \mathcal{F}$ is of the form $f: \wp\left(\Sigma^{*}\right)^{|\mathcal{V}|} \rightarrow \wp\left(\Sigma^{*}\right)^{|\mathcal{V}|}$ and satisfies $\rho\left(\overrightarrow{\boldsymbol{b}} \cup \mathrm{Fn}_{\mathcal{G}}(\overrightarrow{\boldsymbol{X}})\right)=\rho(f(\overrightarrow{\boldsymbol{X}}))$. Then, for all $0 \leq n$,

$$
\left(\rho\left(\vec{b} \cup \operatorname{Fn}_{\mathcal{G}}(\vec{X})\right)^{n}=\rho\left(\mathcal{F}^{n}(\vec{X})\right) .\right.
$$

Proof. We proceed by induction in $n$.

- Base case: Let $n=0$. Then $\mathcal{F}^{0}(\overrightarrow{\boldsymbol{X}})=\left(\rho\left(\overrightarrow{\boldsymbol{b}} \cup \mathrm{Fn}_{\mathcal{G}}(\overrightarrow{\boldsymbol{X}})\right)^{0}=\overrightarrow{\boldsymbol{\varnothing}}\right.$.

- Inductive step: Assume that $\rho\left(\mathcal{F}^{n}(\vec{X})\right)=\left(\rho\left(\vec{b} \cup \mathrm{Fn}_{\mathcal{G}}(\vec{X})\right)^{n}\right.$ holds for some value $n \geq 0$. To simplify the notation, let $\mathcal{P}(\vec{X})=\vec{b} \cup \mathrm{Fn}_{\mathcal{G}}(\vec{X})$ so that $\rho \mathcal{F}^{n}=(\rho \mathcal{P})^{n}$. Then

$$
\begin{aligned}
\rho \mathcal{F}^{n+1}(\overrightarrow{\boldsymbol{X}})= & {\left[\text { Since } \mathcal{F}^{n+1}=\mathcal{F}^{n} \mathcal{F}\right] } \\
\rho \mathcal{F}^{n} \mathcal{F}(\overrightarrow{\boldsymbol{X}})= & {[\text { By Inductive Hypothesis }] } \\
(\rho \mathcal{P})^{n} \mathcal{F}(\overrightarrow{\boldsymbol{X}})= & {[\text { By Theorem 4.5.2, } \rho \text { is bw. complete for } \mathcal{P}] } \\
(\rho \mathcal{P})^{n} \rho \mathcal{F}(\overrightarrow{\boldsymbol{X}})= & {[\text { Since } \rho f=\rho \mathcal{P}] } \\
(\rho \mathcal{P})^{n} \rho \mathcal{P}(\overrightarrow{\boldsymbol{X}})= & {\left[\text { By definition of }(\rho(\mathcal{P}))^{n}\right] } \\
(\rho \mathcal{P})^{n+1}(\overrightarrow{\boldsymbol{X}}) &
\end{aligned}
$$

We conclude that $\left(\rho\left(\overrightarrow{\boldsymbol{b}} \cup \mathrm{Fn}_{\mathcal{G}}(\overrightarrow{\boldsymbol{X}})\right)^{n}=\rho\left(\mathcal{F}^{n}(\overrightarrow{\boldsymbol{X}})\right)\right.$ for all $0 \leq n$.

We are now in position to show that the procedure $\overline{\operatorname{KLEENE}}(\mathrm{AbsEq}, \mathcal{F}, b)$ can be used to compute $\operatorname{lfp}\left(\lambda \overrightarrow{\boldsymbol{X}} . \rho\left(\overrightarrow{\boldsymbol{b}} \cup \mathrm{Fn}_{\mathcal{G}}(\overrightarrow{\boldsymbol{X}})\right)\right)$.

LEMMA 4.5.4. Let $\rho \in \operatorname{uco}\left(\Sigma^{*}\right)$ be backward complete for $\lambda X \in \wp\left(\Sigma^{*}\right)$. aX and $\lambda X \in \wp\left(\Sigma^{*}\right)$.Xa for all $a \in \Sigma$ such that $\left\langle\left\{\rho(S) \mid S \in \wp\left(\Sigma^{*}\right)\right\}, \subseteq\right\rangle$ is an ACC CPO and let $\mathcal{G}=\langle\mathcal{V}, \Sigma, P\rangle$ be a $C F G$ in $C N F$. Let $\mathcal{F}$ be a set offunctions such that every $f \in \mathcal{F}$ is of the form $f: \wp\left(\Sigma^{*}\right)^{|\mathcal{V}|} \rightarrow \wp\left(\Sigma^{*}\right)^{|\mathcal{V}|}$ and satisfies $\rho\left(\overrightarrow{\boldsymbol{b}} \cup \mathrm{Fn}_{\mathcal{G}}(\overrightarrow{\boldsymbol{X}})\right)=\rho(f(\overrightarrow{\boldsymbol{X}}))$. Then,

$$
\operatorname{lfp}\left(\rho\left(\overrightarrow{\boldsymbol{b}} \cup \operatorname{Fn}_{\mathcal{G}}(\overrightarrow{\boldsymbol{X}})\right)\right)=\rho(\widehat{\operatorname{KLEENE}}(\mathrm{AbsEq}, \mathcal{F}, \vec{\varnothing})) .
$$


Moreover, the iterates of $\operatorname{KLEENE}\left(\rho\left(\overrightarrow{\boldsymbol{b}} \cup \mathrm{Fn}_{\mathcal{G}}(\vec{X})\right), \vec{\varnothing}\right)$ coincide in lockstep with the abstraction of the iterates of $\overline{\operatorname{KLEENE}}(\mathrm{AbsEq}, \mathcal{F}, \vec{\varnothing})$

Proof. Since $\left\langle\left\{\rho(S) \mid S \in \wp\left(\Sigma^{*}\right)\right\}, \subseteq\right\rangle$ is an ACC CPO, by Theorem 3.5.1, we have that

$$
\operatorname{lfp}\left(\rho\left(\overrightarrow{\boldsymbol{b}} \cup \operatorname{Fn}_{\mathcal{G}}(\vec{X})\right)\right)=\operatorname{KLEENE}\left(\rho\left(\overrightarrow{\boldsymbol{b}} \cup \operatorname{Fn}_{\mathcal{G}}(\vec{X})\right), \vec{\varnothing}\right)
$$

On the other hand, by Lemma 4.5.3, the iterates of the above Kleene iteration coincide in lockstep with the abstraction of the iterates of $\overline{\operatorname{KLEENE}}(\mathrm{AbsEq}, \mathcal{F}, \vec{\varnothing})$ and, therefore,

$$
\left.\operatorname{KLEENE}\left(\rho\left(\overrightarrow{\boldsymbol{b}} \cup \operatorname{Fn}_{\mathcal{G}}(\vec{X})\right)\right), \vec{\varnothing}\right)=\rho(\widehat{\operatorname{KLEENE}}(\mathrm{AbsEq}, \mathcal{F}, \vec{\varnothing}))
$$

As a consequence,

$$
\operatorname{lfp}\left(\rho\left(\overrightarrow{\boldsymbol{b}} \cup \operatorname{Fn}_{\mathcal{G}}(\vec{X})\right)\right)=\rho(\widehat{\operatorname{KLEENE}}(\mathrm{AbsEq}, \mathcal{F}, \vec{\varnothing}))
$$

We are now in position to introduce the equivalent of Theorem 4.2.10 for grammars.

THEOREM 4.5.5. Let $\mathcal{G}=\langle\mathcal{V}, \Sigma, P\rangle$ be a $C F G$ in $C N F$, let $L_{2}$ be a regular language, let $\rho \in \operatorname{uco}\left(\Sigma^{*}\right)$ and let $\mathcal{F}$ be a set of functions. Assume that the following properties hold:

(i) The abstraction $\rho$ satisfies $\rho\left(L_{2}\right)=L_{2}$ and it is backward complete for both $\lambda X \in \wp\left(\Sigma^{*}\right)$. $a X$ and $\lambda X \in \wp\left(\Sigma^{*}\right)$.Xa for all $a \in \Sigma$.

(ii) The set $\left\langle\left\{\rho(S) \mid S \in \wp\left(\Sigma^{*}\right)\right\}\right.$, $\left.\subseteq\right\rangle$ is an ACC CPO.

(iii) Every function $f_{i}$ in the set $\mathcal{F}$ is of the form $f_{i}: \wp\left(\Sigma^{*}\right)^{|\mathcal{V}|} \rightarrow \wp\left(\Sigma^{*}\right)^{|\mathcal{V}|}$, it is computable and satisfies $\rho\left(\overrightarrow{\boldsymbol{b}} \cup \mathrm{Fn}_{\mathcal{G}}(\vec{X})\right)=\rho\left(f_{i}(\vec{X})\right)$.

(iv) There is an algorithm, say $\operatorname{AbsEq}^{\sharp}(\vec{X}, \vec{Y})$, which decides the abstraction equivalence $\rho(\vec{X})=\rho(\vec{Y})$, for all $\vec{X}, \vec{Y} \in \wp\left(\Sigma^{*}\right)^{|\mathcal{V}|}$.

(v) There is an algorithm, say $\operatorname{Incl}^{\sharp}(\vec{X})$, which decides the inclusion $\rho(\vec{X}) \subseteq \overrightarrow{L_{2}} X_{0}$, for all $\vec{X} \in \wp\left(\Sigma^{*}\right)^{|\mathcal{V}|}$.

Then, the following is an algorithm which decides whether $\mathcal{L}(\mathcal{G}) \subseteq L_{2}$ :

$\left\langle Y_{i}\right\rangle_{i \in[0, n]}:=\overline{\operatorname{KLEENE}}\left(\mathrm{AbsEq}^{\sharp}, \mathcal{F}, \vec{\varnothing}\right) ;$

return $\operatorname{Incl}^{\sharp}\left(\left\langle Y_{i}\right\rangle_{i \in[0, n]}\right)$;

Proof. It follows from hypotheses (i), (ii) and (iii), by Lemma 4.5.4, that

$$
\operatorname{lfp}\left(\rho\left(\overrightarrow{\boldsymbol{\epsilon}}^{F} \cup \operatorname{Pre}_{\mathcal{N}}(\vec{X})\right)\right)=\rho(\widehat{\operatorname{KLEENE}}(\operatorname{AbsEq}, \mathcal{F}, \vec{\varnothing}))
$$


Observe that function AbsEq can be replaced by function AbsEq ${ }^{\#}$ due to hypothesis (iv). Moreover, it follows from Equivalence (4.20), which holds by hypothesis (i), and Equation (4.21) that

$$
\mathcal{L}(\mathcal{N}) \subseteq L_{2} \Leftrightarrow \rho\left(\widehat{\operatorname{KLEENE}}\left(\mathrm{AbsEq}^{\sharp}, \mathcal{F}, \vec{\varnothing}\right)\right) \subseteq \overrightarrow{\boldsymbol{L}_{2}} X_{0}
$$

Finally, hypotheses (iv) and (v) guarantee, respectively, the decidability of the inclusion $\rho \mathcal{F}(X) \subseteq \rho(X)$ performed at each step of the $\overline{\text { KLEENE }}$ iteration and the decidability of the inclusion of the lfp in $\overrightarrow{L_{2}} X_{0}$.

\subsubsection{Solving the Abstract Inclusion Check using Galois Connec- tions}

The following result is the equivalent of Theorem 4.2.11 for context-free languages. It shows that the language inclusion problem $\mathcal{L}(\mathcal{G}) \subseteq L_{2}$ can be solved by working on an abstract domain.

TheOREM 4.5.6. Let $\mathcal{G}=\langle\mathcal{V}, \Sigma, P\rangle$ be a $C F G$ in $C N F$ and let $L_{2}$ be a language over $\sum$. Let $\left\langle\wp\left(\Sigma^{*}\right), \subseteq\right\rangle \underset{\alpha}{\stackrel{\gamma}{\leftrightarrows}}\langle D, \sqsubseteq\rangle$ be a $G C$ where $\left\langle D, \leq_{D}\right\rangle$ is a poset. Assume that the following properties hold:

(i) $L_{2} \in \gamma(D)$ and for every $a \in \Sigma, X \in \wp\left(\Sigma^{*}\right), \gamma \alpha(a X)=\gamma \alpha(a \gamma \alpha(X))$ and $\gamma \alpha(X a)=\gamma \alpha \gamma(\alpha(X) a)$.

(ii) $\left(D, \leq_{D}, \sqcup, \perp_{D}\right)$ is an effective domain, meaning that: $\left(D, \leq_{D}, \sqcup, \perp_{D}\right)$ is an ACC join-semilattice with bottom $\perp_{D}$, every element of $D$ has a finite representation, the binary relation $\leq_{D}$ is decidable and the binary lub $\sqcup$ is computable.

(iii) There is an algorithm, say $\operatorname{Fn}^{\sharp}\left(\vec{X}^{\sharp}\right)$, which computes $\alpha\left(\operatorname{Fn}_{\mathcal{G}}(\gamma(\vec{X}))\right)$, for all $\vec{X}^{\sharp} \in \alpha\left(\wp\left(\Sigma^{*}\right)\right)^{|\mathcal{V}|}$.

(iv) There is an algorithm, say $b^{\sharp}$, which computes $\alpha(\vec{b})$.

(v) There is an algorithm, say $\operatorname{Inc} \mathbb{1}^{\sharp}\left(\vec{X}^{\sharp}\right)$, which decides the abstract inclusion $\vec{X}^{\sharp} \leq_{D} \alpha\left(\overrightarrow{L_{2}} X_{0}\right)$, for all $\vec{X}^{\sharp} \in \alpha\left(\wp\left(\Sigma^{*}\right)^{|\mathcal{V}|}\right)$.

Then, the following is an algorithm which decides whether $\mathcal{L}(\mathcal{G}) \subseteq L_{2}$ :

$$
\left\langle Y_{i}^{\sharp}\right\rangle_{i \in[0, n]}:=\operatorname{KLEENE}\left(\lambda \vec{X}^{\sharp} \cdot b^{\sharp} \sqcup \mathrm{Fn}^{\sharp}\left(\vec{X}^{\sharp}\right), \overrightarrow{\perp_{D}}\right) ;
$$

return $\operatorname{Incl}^{\sharp}\left(\left\langle Y_{i}^{\sharp}\right\rangle_{i \in[0, n]}\right)$; 
Proof. Let $\rho=\gamma \alpha \in \operatorname{uco}\left(\wp\left(\Sigma^{*}\right)\right)$. Then, it follows from property (i) that $L_{2} \in \rho$, $\rho(a X)=\rho(a \rho(X))$ and $\rho(X a)=\rho(\rho(X) a)$. Therefore

$$
\begin{aligned}
\mathcal{L}(\mathcal{N}) \subseteq L_{2} \Leftrightarrow & {[\text { By (4.20)] }} \\
\operatorname{lfp}\left(\lambda \overrightarrow{\boldsymbol{X}} \cdot \rho\left(\overrightarrow{\boldsymbol{b}} \cup \operatorname{Fn}_{\mathcal{G}}(\overrightarrow{\boldsymbol{X}})\right)\right) \subseteq{\overrightarrow{\boldsymbol{L}_{2}}}^{X_{0}} \Leftrightarrow & {[\text { By Lemma 3.6.2] }} \\
\gamma\left(\operatorname{lfp}\left(\lambda \overrightarrow{\boldsymbol{X}^{\sharp}} \cdot \alpha(\overrightarrow{\boldsymbol{b}}) \sqcup \alpha\left(\operatorname{Fn}_{\mathcal{G}}\left(\gamma\left(\overrightarrow{\boldsymbol{X}^{\sharp}}\right)\right)\right)\right) \subseteq{\overrightarrow{\boldsymbol{L}_{2}}}^{X_{0}} \Leftrightarrow\right. & {\left[\text { By GC and since } L_{2} \in \rho\right] } \\
\operatorname{lfp}\left(\lambda \overrightarrow{\boldsymbol{X}}^{\sharp} \cdot \alpha(\overrightarrow{\boldsymbol{b}}) \sqcup \alpha\left(\operatorname{Fn}_{\mathcal{G}}\left(\gamma\left(\overrightarrow{\boldsymbol{X}}^{\sharp}\right)\right)\right)\right) \leq_{D} \alpha\left({\overrightarrow{\boldsymbol{L}_{2}}}^{X_{0}}\right) . &
\end{aligned}
$$

By hypotheses (ii), (iii) and (iv) it turns out that $\operatorname{KLEENE}\left(\lambda \vec{X}^{\sharp} \cdot b^{\sharp} \sqcup \mathrm{Fn}^{\sharp}\left(\vec{X}^{\sharp}\right), \overrightarrow{\perp_{D}}\right)$ is an algorithm computing $\operatorname{lfp}\left(\lambda \vec{X}^{\sharp} . \alpha(\vec{b}) \sqcup \alpha\left(\operatorname{Fn}_{\mathcal{G}}\left(\gamma\left(\vec{X}^{\sharp}\right)\right)\right)\right)$. In particular, these hypotheses ensure that the Kleene iterates of $\operatorname{lfp}\left(\lambda \vec{X}^{\sharp} . \alpha(\vec{b}) \sqcup \alpha\left(\operatorname{Fn}_{\mathcal{G}}\left(\gamma\left(\vec{X}^{\sharp}\right)\right)\right)\right)$ starting from $\overrightarrow{\perp_{D}}$ are computable, finitely many and that it is decidable whether the iterates have reached the fixpoint. The hypothesis (v) ensures decidability of the required $\leq_{D}$-inclusion check of this least fixpoint in $\alpha\left(\wp\left(\Sigma^{*}\right)\right)^{|\mathcal{V}|}$.

\subsubsection{Instantiating the Framework}

Let us instantiate the general algorithmic framework provided by Theorem 4.5.5 to the class of closure operators induced by quasiorder relations on words. Recall that a quasiorder $\leqslant$ on $\Sigma^{*}$ is monotone if

$$
\forall x_{1}, x_{2} \in \Sigma^{*}, \forall a, b \in \Sigma, x_{1} \leqslant x_{2} \Rightarrow a x_{1} b \leqslant a x_{2} b .
$$

It follows that $x_{1} \leqslant x_{2} \Rightarrow \forall u, v \in \Sigma^{*}, u x_{1} v \leqslant u x_{2} v$. The following result is the equivalent to Lemma 4.3.2 for $L$-consistent quasiorders and it allows us to characterize $L$-consistent quasiorders in terms of the induced closure.

LеммA 4.5.7. Let $L \in \wp\left(\Sigma^{*}\right)$ and $\leqslant_{L}$ be a quasiorder on $\Sigma^{*}$. Then, $\leqslant_{L}$ is an $L$ consistent quasiorder on $\Sigma^{*}$ if and only if

(a) $\rho_{\leqslant_{L}}(L)=L$, and

(b) $\rho_{\leqslant_{L}}$ is backward complete for $\lambda X$. aXb for all $a, b \in \Sigma$.

Proof.

(a) It follows from Lemma 4.3.2 (a) since, by Definition 4.3.1, a quasiorder is $L$-consistent iff it is left and right $L$-consistent.

(b) We first prove that if $\leqslant_{L}$ is monotone. Then for all $X \in \wp\left(\Sigma^{*}\right)$ we have that $\rho_{\leqslant_{L}}(a X b)=\rho_{\leqslant_{L}}\left(a \rho_{\leqslant_{L}}(X) b\right)$ for all $a, b \in \Sigma$.

Monotonicity of concatenation together with monotonicity and extensivity of the closure $\rho_{\leqslant_{L}}$ imply that $\rho_{\leqslant_{L}}(a X b) \subseteq \rho_{\leqslant_{L}}\left(a \rho_{\leqslant_{L}}(X) b\right)$ holds. For 
the reverse inclusion, we have that:

$$
\begin{aligned}
\rho_{\leqslant_{L}}\left(a \rho_{\leqslant_{L}}(X) b\right) & =\text { [By definition of } \rho_{\leqslant_{L}} \text { ] } \\
\rho_{\leqslant_{L}}\left(\left\{a y b \mid \exists x \in X, x \leqslant_{L} y\right\}\right) & =\text { [By definition of } \rho_{\leqslant_{L}} \text { ] } \\
\left\{z \mid \exists x \in X, y \in \Sigma^{*}, x \leqslant_{L} y \wedge a y b \leqslant_{L} z\right\} & \subseteq \text { [By monotonicity of } \leqslant_{L} \text { ] } \\
\left\{z \mid \exists x \in X, y \in \Sigma^{*}, a x b \leqslant_{L} a y b \wedge a y b \leqslant_{L} z\right\} & \left.=\text { [By transitivity of } \leqslant_{L}\right] \\
\left\{z \mid \exists x \in X, a x b \leqslant_{L} z\right\} & =\text { [By definition of } \rho_{\leqslant_{L}} \text { ] } \\
\rho_{\leqslant_{L}}(a X b) & .
\end{aligned}
$$

Next, we show that if $\rho_{\leqslant_{L}}(a X b)=\rho_{\leqslant_{L}}\left(a \rho_{\leqslant_{L}}(X) b\right)$ for all $X \in \wp\left(\Sigma^{*}\right)$ and $a, b \in \sum$ then $\leqslant_{L}$ is monotone. Let $x_{1}, x_{2} \in \Sigma^{*}, a, b \in \sum$. If $x_{1} \leqslant_{L} x_{2}$ then $\left\{x_{2}\right\} \subseteq \rho_{\leqslant_{L}}\left(\left\{x_{1}\right\}\right)$, and in turn $a\left\{x_{2}\right\} b \subseteq a \rho_{\leqslant_{L}}\left(\left\{x_{1}\right\}\right) b$. Since $\rho_{\leqslant_{L}}$ is monotone, we have that $\rho_{\leqslant_{L}}\left(a\left\{x_{2}\right\} b\right) \subseteq \rho_{\leqslant_{L}}\left(a \rho_{\leqslant_{L}}\left(\left\{x_{1}\right\}\right) b\right)$, so that, by backward completeness, $\rho_{\leqslant_{L}}\left(a\left\{x_{2}\right\} b\right) \subseteq \rho_{\leqslant_{L}}\left(a\left\{x_{1}\right\} b\right)$. It follows that, $a\left\{x_{2}\right\} b \subseteq \rho_{\leqslant_{L}}\left(a\left\{x_{1}\right\} b\right)$, namely, $a x_{1} b \leqslant_{L} a x_{2} b$. By Equation (4.22), this shows that $\leqslant_{L}$ is monotone.

Analogously to the case of regular languages presented in Section 4.3, Theorem 4.5.5 induces an algorithm for deciding the language inclusion $\mathcal{L}(\mathcal{G}) \subseteq L_{2}$ for any CFG $\mathcal{G}$ and regular language $L_{2}$. Indeed, we can apply Theorem 4.5 .5 with $\left\lfloor\vec{b} \cup \operatorname{Fn}_{\mathcal{G}}(\vec{X})\right\rfloor$ interpreted as the set of functions $f_{i} \stackrel{\text { def }}{=}\left\lfloor\overrightarrow{\boldsymbol{b}} \cup \mathrm{Fn}_{\mathcal{G}}(\vec{X})\right\rfloor_{i}$ where, again, each $\lfloor\cdot\rfloor_{i}$ is a function mapping each set $X \in \wp\left(\Sigma^{*}\right)$ into a minor $\lfloor X\rfloor_{i}$.

As a consequence, we obtain Algorithm CFGINCW which, given a language $L_{2}$ whose membership problem is decidable and a decidable $L_{2}$-consistent wellquasiorder, determines whether $\mathcal{L}(\mathcal{G}) \subseteq L_{2}$ holds.

CFGIncW: Word-based algorithm for $\mathcal{L}(\mathcal{G}) \subseteq L_{2}$

Data: $C F G G=\langle\mathcal{V}, \Sigma, P\rangle$; decision procedure for $u \in L_{2}$; decidable $L_{2}$-consistent wqo $\leqslant L_{2}$.

$\mathbf{1}\left\langle Y_{i}\right\rangle_{i \in[0, n]}:=\widehat{\operatorname{KLEENE}}\left(\sqsubseteq_{L_{2}} \cap\left(\sqsubseteq_{L_{L}}\right)^{-1}, \lambda \overrightarrow{\boldsymbol{X}} \cdot\left\lfloor\overrightarrow{\boldsymbol{b}} \cup \mathrm{Fn}_{\mathcal{G}}(\vec{X})\right\rfloor, \vec{\varnothing}\right) ;$

2 forall $u \in Y_{0}$ do

3 if $u \notin L_{2}$ then return false;

4 return true;

TheOREM 4.5.8. Let $\mathcal{G}=\langle\mathcal{V}, \Sigma, P\rangle$ be a $C F G$ in $C N F$ and let $L_{2} \in \wp\left(\Sigma^{*}\right)$ be a language such that: (i) membership $u \in L_{2}$ is decidable; (ii) there exists a decidable $L_{2}$-consistent well-quasiorder on $\Sigma^{*}$. Then, Algorithm CFGINCW decides the inclusion $\mathcal{L}(\mathcal{G}) \subseteq L_{2}$. 
Proof. Let $\leqslant_{L_{2}}$ be a decidable $L_{2}$-consistent well-quasiorder on $\Sigma^{*}$. Then, we check that hypotheses (i)-(v) of Theorem 4.5.5 are satisfied.

(a) It follows from hypothesis (ii) and Lemma 4.5.7 that $\leqslant_{L_{2}}$ is backward complete for left and right concatenation and satisfies $\rho_{\leqslant_{L_{2}}}\left(L_{2}\right)=L_{2}$.

(b) Since $\leqslant_{L_{2}}$ is a well-quasiorder, it follows that $\left\langle\left\{\rho_{\leqslant_{L_{2}}}(S) \mid S \in \wp\left(\Sigma^{*}\right)\right\}, \subseteq\right\rangle$ is an ACC CPO.

(c) Let $\left\lfloor\vec{b} \cup \mathrm{Fn}_{\mathcal{G}}(\vec{X})\right\rfloor$ be the set of functions $f_{i}$ each of which maps each set $X \in \wp\left(\Sigma^{*}\right)$ into a minor of $\overrightarrow{\boldsymbol{b}} \cup \mathrm{Fn}_{\mathcal{G}}(\vec{X})$. Since $\rho_{\leqslant_{L_{2}}}(X)=\rho_{\leqslant_{L_{2}}}(\lfloor X\rfloor)$ for all $X \in \wp\left(\Sigma^{*}\right)^{|\mathcal{V}|}$, we have that all functions $f_{i}$ satisfy satisfy

$$
\rho\left(\overrightarrow{\boldsymbol{b}} \cup \operatorname{Fn}_{\mathcal{G}}(\rho(\overrightarrow{\boldsymbol{X}}))\right)=\rho\left(f_{i}(\overrightarrow{\boldsymbol{X}})\right)
$$

(d) The equality $\rho_{\leqslant_{L_{2}}}\left(S_{1}\right)=\rho_{\leqslant_{L_{2}}}\left(S_{2}\right)$ is decidable for every $S_{1}, S_{2} \in \wp\left(\Sigma^{*}\right)^{|\mathcal{V}|}$ since $\rho_{\leqslant_{L_{2}}}\left(S_{1}\right)=\rho_{\leqslant_{L_{2}}}\left(S_{2}\right) \Leftrightarrow S_{1} \sqsubseteq_{L_{2}} S_{2} \wedge S_{2} \sqsubseteq_{L_{2}} S_{1}$ and $\leqslant_{L_{2}}$ is decidable.

\left. (e) Since ${\overrightarrow{L_{2}}}^{X_{0}}=\left\langle\psi_{\Sigma^{*}}^{L_{2}}(i=0)\right\rangle_{i \in[0, n]}\right)$, the inclusion trivially holds for all components $Y_{i}$ with $i \neq 0$. Therefore, it suffices to check whether $Y_{0} \subseteq L_{2}$ holds. Since $Y_{0}=\lfloor S\rfloor$ for some set $S \in \wp\left(\Sigma^{*}\right)$, the inclusion $Y_{0} \subseteq L_{2}$ can be decided by performing finitely many membership tests, which is exactly the check performed by lines 2-4 of Algorithm CFGIncW. By hypothesis (i), this check is decidable.

\subsubsection{Myhill and State-based Quasiorders}

In the following, we will consider two quasiorders on $\Sigma^{*}$ and we will show that they fulfill the requirements of Theorem 4.5.8, so that they yield algorithms for deciding the inclusion $\mathcal{L}(\mathcal{G}) \subseteq L_{2}$ for every CFG $\mathcal{G}$ and regular language $L_{2}$.

The context of a word $w \in \Sigma^{*}$ w.r.t a given language $L \in \wp\left(\Sigma^{*}\right)$ is defined as:

$$
\operatorname{ctx}_{L}(w) \stackrel{\text { def }}{=}\left\{(u, v) \in \Sigma^{*} \times \Sigma^{*} \mid u w v \in L\right\} .
$$

Correspondingly, let us define the following quasiorder relation on $\Sigma^{*}$ :

$$
u \leqslant L v \stackrel{\text { def }}{\Leftrightarrow} \operatorname{ctx}_{L}(u) \subseteq \operatorname{ctx}_{L}(v)
$$

de Luca and Varricchio [1994, Section 2] call $\leqslant_{L}$ the Myhill quasiorder relative to $L$. The following result is the analogue of Lemma 4.3.5 for L-consistent and Myhill's quasiorders: it shows that the Myhill's quasiorder is the weakest (i.e. greatest w.r.t. set inclusion between binary relations) $L$-consistent quasiorder for which Algorithm CFGINCW can be instantiated to decide the inclusion $\mathcal{L}(\mathcal{G}) \subseteq L$. 


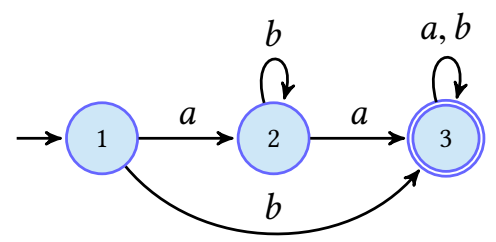

Figure 4.3: A finite automaton $\mathcal{N}$ with $\mathcal{L}(\mathcal{N})=\left(b+a b^{*} a\right)(a+b)^{*}$.

LEMMA 4.5.9. Let $L \in \wp\left(\Sigma^{*}\right)$.

(a) $\leqslant_{L}$ is an L-consistent quasiorder. If $L$ is regular then, additionally, $\leqslant_{L}$ is a decidable well-quasiorder.

(b) If $\leqslant$ is an L-consistent quasiorder on $\Sigma^{*}$ then $\rho_{\leqslant L} \subseteq \rho_{\leqslant}$.

Proof. The proof follows the same lines of the proof of Lemma 4.3.5.

(a) de Luca and Varricchio [1994, Section 3] observe that $\leqslant_{L}$ is monotone. Moreover, if $L$ is regular then $\leqslant_{L}$ is a wqo [de Luca and Varricchio 1994, Proposition 2.3]. Let us observe that given $u \in L$ and $v \notin L$ we have that $(\epsilon, \epsilon) \in \operatorname{ctx}_{L}(u)$ while $(\epsilon, \epsilon) \notin \operatorname{ctx}_{L}(v)$. Hence, $\leqslant L \cap\left(L \times L^{c}\right)=\varnothing$ and, therefore, $\leqslant_{L}$ is an $L$-consistent quasiorder.

Finally, if $L$ is regular then $\leqslant_{L}$ is clearly decidable.

(b) As shown by de Luca and Varricchio [1994], $\leqslant_{L}$ is maximum in the set of all $L$-consistent quasiorders, i.e. every $L$-consistent quasiorder $\leqslant$ is such that $x \leqslant y \Rightarrow x \leqslant_{L} y$. As a consequence, $\rho_{\leqslant}(X) \subseteq \rho_{\leqslant_{L}}(X)$ holds for all $X \in \wp\left(\Sigma^{*}\right)$, namely $\leqslant \subseteq \leqslant{ }_{L}$.

Example 4.5.10. Let us illustrate the use of the Myhill quasiorder $\leqslant \mathcal{L}(\mathcal{N})$ in Algorithm CFGINCW for solving the language inclusion $\mathcal{L}(\mathcal{G}) \subseteq \mathcal{L}(\mathcal{N})$, where $\mathcal{G}$ is the CFG in Example 4.5.1 and $\mathcal{N}$ is the NFA depicted in Figure 4.3. Recall that the equations for $\mathcal{G}$ are:

$$
\operatorname{Eqn}(\mathcal{G})=\left\{\begin{array}{l}
X_{0}=X_{0} X_{1} \cup X_{1} X_{0} \cup\{b\} \\
X_{1}=\{a\}
\end{array}\right.
$$

We write $\{(S, T)\} \cup\{(X, Y)\}$ to denote the set $\{(u, v) \mid(u, v) \in S \times T \cup X \times Y\}$. Then, we have the following contexts (among others) for $L=\mathcal{L}(\mathcal{N})=\left(b+a b^{*} a\right)(a+b)^{*}$ :

$$
\begin{aligned}
& \operatorname{ctx}_{L}(\epsilon)=\{(\epsilon, L)\} \cup\left\{\left(a b^{*}, b^{*} a \Sigma^{*}\right)\right\} \cup\left\{\left(L, \Sigma^{*}\right)\right\} \\
& \operatorname{ctx}_{L}(a)=\left\{\left(\epsilon, b^{*} a \Sigma^{*}\right)\right\} \cup\left\{a b^{*}, \Sigma^{*}\right\} \cup\left\{\left(L, \Sigma^{*}\right)\right\}
\end{aligned}
$$




$$
\begin{aligned}
\operatorname{ctx}_{L}(b) & =\left\{\left(\epsilon, \Sigma^{*}\right)\right\} \cup\left\{\left(a b^{*}, b^{*} a \Sigma^{*}\right)\right\} \cup\left\{\left(L, \Sigma^{*}\right)\right\} \\
\operatorname{ctx}_{L}(b a) & =\left\{\left(\epsilon, \Sigma^{*}\right)\right\} \cup\left\{\left(a b^{*}, \Sigma^{*}\right)\right\} \cup\left\{\left(L, \Sigma^{*}\right)\right\}
\end{aligned}
$$

Moreover, $\operatorname{ctx}_{L}(a b)=\operatorname{ctx}_{L}(a)$ and $\operatorname{ctx}_{L}(b a)=\operatorname{ctx}_{L}(a a)=\operatorname{ctx}_{L}(a a a)=\operatorname{ctx}_{L}(a a b)=$ $\operatorname{ctx}_{L}(a b a)$ and, since $a \leqslant_{L} b a$ and $\varepsilon \leqslant_{L} b$, it follows that $\left\lfloor\Sigma^{*}\right\rfloor=\{\epsilon, a\}$.

Recall that, as shown in Example 4.5.1, we have that $\vec{b}=\langle\{b\},\{a\}\rangle$ and $\operatorname{Fn}_{\mathcal{G}}\left(\left\langle X_{0}, X_{1}\right\rangle\right)=\left\langle X_{0} X_{1} \cup X_{1} X_{0}, \varnothing\right\rangle$. Next, we show the computation of the Kleene iterates according to Algorithm CFGINCW when using the quasiorder $\leqslant_{L}$.

$$
\begin{aligned}
\vec{Y}^{(0)} & =\vec{\varnothing} \\
\vec{Y}^{(1)} & =\lfloor\overrightarrow{\boldsymbol{b}}\rfloor=\langle\{b\},\{a\}\rangle \\
\overrightarrow{\boldsymbol{Y}}^{(2)} & =\lfloor\overrightarrow{\boldsymbol{b}}\rfloor \sqcup\left\lfloor\operatorname{Fn}_{\mathcal{G}}\left(\vec{Y}^{(1)}\right)\right\rfloor=\langle\{b\},\{a\}\rangle \sqcup\langle\lfloor\{b a, a b\}\rfloor,\lfloor\varnothing\rfloor\rangle \\
& =\langle\lfloor\{b a, a b, b\}\rfloor,\lfloor\{a\}\rfloor\rangle=\langle\{a b, b\},\{a\}\rangle \\
\vec{Y}^{(3)} & =\lfloor\overrightarrow{\boldsymbol{b}}\rfloor \sqcup\left\lfloor\mathrm{Fn}_{\mathcal{G}}\left(\vec{Y}^{(2)}\right)\right\rfloor=\langle\{b\},\{a\}\rangle \sqcup\langle\lfloor\{a b a, b a, a a b, a b\}\rfloor,\lfloor\varnothing\rfloor\rangle \\
& =\langle\lfloor\{a b a, b a, a a b, a b, b\}\rfloor,\lfloor\{a\}\rfloor\rangle=\langle\{a b, b\},\{a\}\rangle
\end{aligned}
$$

The least fixpoint is therefore $\vec{Y}=\langle\{a b, b\},\{a\}\rangle$. Since $a b \in \vec{Y}_{0}$ but $a b \notin \mathcal{L}(\mathcal{N})$, Algorithm CFGINCW concludes that the inclusion $\mathcal{L}(\mathcal{G}) \subseteq \mathcal{L}(\mathcal{N})$ does not hold. $\diamond$

Similarly to Section 4.3 , we also consider a state-based quasiorder that can be used with Algorithm CFGINcW. First, given an NFA $\mathcal{N}=\langle Q, \delta, I, F, \Sigma\rangle$ we define the state-based equivalent of the context of a word $w \in \Sigma^{*}$ as follows:

$$
\operatorname{ctx}_{\mathcal{N}}(w) \stackrel{\text { def }}{=}\left\{\left(q, q^{\prime}\right) \in Q \times Q \mid q \stackrel{w}{\sim} q^{\prime}\right\} .
$$

Then, the quasiorder $\leqslant_{\mathcal{N}}$ on $\Sigma^{*}$ is defined as follows: for all $u, v \in \Sigma^{*}$,

$$
u \leqslant \leqslant_{\mathcal{N}} v \stackrel{\text { def }}{\Leftrightarrow} \operatorname{ctx}_{\mathcal{N}}(u) \subseteq \operatorname{ctx}_{\mathcal{N}}(v)
$$

The following result is the analogue of Lemma 4.3.7 and shows that $\leqslant_{\mathcal{N}}$ is a $\mathcal{L}(\mathcal{N})$-consistent well-quasiorder, hence it can be used with Algorithm CFGINcW to decide the language inclusion $\mathcal{L}(\mathcal{G}) \subseteq \mathcal{L}(\mathcal{N})$.

LEMMA 4.5.11. The relation $\leqslant_{\mathcal{N}}$ is a decidable $\mathcal{L}(\mathcal{N})$-consistent wqo.

Proof. For every $u \in \Sigma^{*}, \operatorname{ctx}_{\mathcal{N}}(u)$ is a finite and computable set, so that $\leqslant_{\mathcal{N}}$ is a decidable wqo. Next, we show that $\leqslant_{\mathcal{N}}$ is $\mathcal{L}(\mathcal{N})$-consistent according to Definition 4.3.1 (a)-(b).

(a) By picking $u \in \mathcal{L}(\mathcal{N})$ and $v \notin \mathcal{L}(\mathcal{N})$ we have that $\operatorname{ctx}_{\mathcal{N}}(u)$ contains a pair $\left(q_{i}, q_{f}\right)$ with $q_{i} \in I$ and $q_{f} \in F$ while $\operatorname{ctx}_{\mathcal{N}}(v)$ does not, hence $u \Varangle_{\mathcal{N}} v$. Therefore, $\leqslant \mathcal{N} \cap\left(\mathcal{L}(\mathcal{N}) \times \mathcal{L}(\mathcal{N})^{c}\right)=\varnothing$. 
(b) Let us check that $\leqslant_{\mathcal{N}}$ is monotone. To do that, first observe that function $\operatorname{ctx}_{\mathcal{N}}:\left\langle\Sigma^{*}, \leqslant_{\mathcal{N}}\right\rangle \rightarrow\left\langle\wp\left(Q^{2}\right), \subseteq\right\rangle$ is monotone. Therefore, for all $x_{1}, x_{2} \in \Sigma^{*}$ and $a, b \in \sum$ we have that

$$
\begin{aligned}
x_{1} \leqslant_{\mathcal{N}} x_{2} & \Rightarrow & {[\text { By def. of } \leqslant \mathcal{N}] } \\
\operatorname{ctx}_{\mathcal{N}}\left(x_{1}\right) \subseteq \operatorname{ctx}_{\mathcal{N}}\left(x_{2}\right) & \Rightarrow & {\left[\text { Since ctx } \mathcal{N}_{\mathcal{N}} \text { is monotone }\right] } \\
\operatorname{ctx}_{\mathcal{N}}\left(a x_{1} b\right) \subseteq \operatorname{ctx}_{\mathcal{N}}\left(a x_{2} b\right) & \Rightarrow & {\left[\text { By def. of } \leqslant_{\mathcal{N}}\right] } \\
a x_{1} b \leqslant_{\mathcal{N}} a x_{2} b & &
\end{aligned}
$$

For the Myhill wqo $\leqslant \mathcal{L}(\mathcal{N})$, it turns out that for all $u, v \in \Sigma^{*}$,

$$
u \leqslant \mathcal{L}(\mathcal{N}) v \Leftrightarrow \underset{\operatorname{ctx}_{\mathcal{L}(\mathcal{N})}(v)}{\subseteq} \begin{gathered}
\operatorname{ctx}_{\mathcal{L}(\mathcal{N})}(u) \\
\left\{(x, y) \mid x \in W_{I, q} \wedge y \in W_{q^{\prime}, F} \wedge q \stackrel{v}{\sim} q^{\prime}\right\}
\end{gathered}
$$

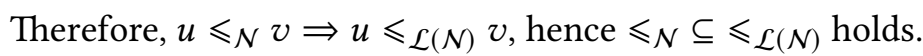

Example 4.5.12. Let us illustrate the use of the state-based quasiorder $\leqslant_{\mathcal{N}}$ to solve the language inclusion $\mathcal{L}(\mathcal{G}) \subseteq \mathcal{L}(\mathcal{N})$ of Example 4.5.10. Here, we have the following contexts (among others):

$$
\begin{aligned}
& \operatorname{ctx}_{\mathcal{N}}(\epsilon)=\left\{\left(q_{1}, q_{1}\right),\left(q_{2}, q_{2}\right),\left(q_{3}, q_{3}\right)\right\} \quad \operatorname{ctx}_{\mathcal{N}}(a)=\left\{\left(q_{1}, q_{2}\right),\left(q_{2}, q_{3}\right),\left(q_{3}, q_{3}\right)\right\} \\
& \operatorname{ctx}_{\mathcal{N}}(b)=\left\{\left(q_{1}, q_{3}\right),\left(q_{2}, q_{2}\right),\left(q_{3}, q_{3}\right)\right\} \quad \operatorname{ctx}_{\mathcal{N}}(a a)=\left\{\left(q_{1}, q_{3}\right),\left(q_{2}, q_{3}\right),\left(q_{3}, q_{3}\right)\right\}
\end{aligned}
$$

Moreover, $\operatorname{ctx}_{\mathcal{N}}(a b)=\operatorname{ctx}_{\mathcal{N}}(a)$ and $\operatorname{ctx}_{\mathcal{N}}(b a)=\operatorname{ctx}_{\mathcal{N}}(a a)=\operatorname{ctx}_{\mathcal{N}}(b a a)=$ $\operatorname{ctx}_{\mathcal{N}}(a a b)=\operatorname{ctx}_{\mathcal{N}}(a b a)$. Recall from Example 4.5.10 that for the Myhill wqo we have that $a \leqslant_{\mathcal{L}(\mathcal{N})} b a$, while for the state-based qo a $\Varangle_{\mathcal{N}} b a$. Next, we show the Kleene iterates computed by Algorithm CFGINCW when using the wqo $\leqslant_{\mathcal{N}}$.

$$
\begin{aligned}
\vec{Y}^{(0)} & =\vec{\varnothing} \\
\vec{Y}^{(1)} & =\lfloor\overrightarrow{\boldsymbol{b}}\rfloor=\langle\{b\},\{a\}\rangle \\
\vec{Y}^{(2)} & =\lfloor\overrightarrow{\boldsymbol{b}}\rfloor \sqcup\left\lfloor\operatorname{Fn}_{\mathcal{G}}\left(\vec{Y}^{(1)}\right)\right\rfloor=\langle\lfloor\{b a, a b, b\}\rfloor,\lfloor\{a\}\rfloor\rangle=\langle\{b a, a b, b\},\{a\}\rangle \\
\overrightarrow{\boldsymbol{Y}}^{(3)} & =\lfloor\overrightarrow{\boldsymbol{b}}\rfloor \sqcup\left\lfloor\operatorname{Fn}_{\mathcal{G}}\left(\vec{Y}^{(2)}\right)\right\rfloor=\langle\lfloor\{a b a, a a b, a b, b a a, a b a, b a, b\}\rfloor,\lfloor\{a\}\rfloor\rangle \\
& =\langle\{b a, a b, b\},\{a\}\rangle
\end{aligned}
$$

The least fixpoint is therefore $\vec{Y}=\langle\{b a, a b, b\},\{a\}\rangle$. Since $a b \in \vec{Y}_{0}$ but $a b \notin \mathcal{L}(\mathcal{N})$, Algorithm CFGINCW concludes that the inclusion $\mathcal{L}(\mathcal{G}) \subseteq \mathcal{L}(\mathcal{N})$ does not hold. $\diamond$ 


\subsubsection{A Systematic Approach to the Antichain Algorithm}

Consider a CFG $\mathcal{G}=\langle\mathcal{V}, \Sigma, P\rangle$ and an NFA $\mathcal{N}=\langle Q, \Sigma, \delta, I, F\rangle$ and let $\leqslant_{\mathcal{N}}$ be the $\mathcal{L}(\mathcal{N})$-consistent wqo defined in (4.24). Theorem 4.5.3 shows that the algorithm CFGINcW solves the inclusion problem $\mathcal{L}(\mathcal{G}) \subseteq \mathcal{L}(\mathcal{N})$ by working with finite languages.

Similarly to the case of the quasiorder $\leqslant_{\mathcal{N}}^{\ell}$ (Section 4.4) it suffices to keep the sets $\operatorname{ctx}_{\mathcal{N}}(u)$ of pairs of states of $Q$ for each word $u$ instead of the words themselves. Therefore, we can systematically derive a "state-based" algorithm analogous to CFGINCW but working on the antichain poset $\left\langle\mathrm{AC}_{\langle\wp(Q \times Q), \subseteq\rangle}, \sqsubseteq\right\rangle$ viewed as an abstraction of $\left\langle\wp\left(\Sigma^{*}\right), \subseteq\right\rangle$. Let us define the abstraction and concretization maps $\alpha: \wp\left(\Sigma^{*}\right) \rightarrow \mathrm{AC}_{\langle\wp(Q \times Q), \subseteq\rangle}$ and $\gamma: \mathrm{AC}_{\langle\wp(Q \times Q), \subseteq\rangle} \rightarrow \wp\left(\Sigma^{*}\right)$ and the abstract function $\operatorname{Fn}_{\mathcal{G}}^{\mathcal{N}}\left(\left\langle X_{i}\right\rangle_{i \in[0, n]}\right): \wp(Q \times Q)^{|\mathcal{V}|} \rightarrow \wp(Q \times Q)^{|\mathcal{V}|}$ as follows:

$$
\begin{aligned}
& \alpha(X) \stackrel{\text { def }}{=}\left\lfloor\left\{\operatorname{ctx}_{\mathcal{N}}(u) \mid u \in X\right\}\right\rfloor \\
& \gamma(Y) \stackrel{\text { def }}{=}\left\{u \in \Sigma^{*} \mid \exists y \in Y, y \subseteq \operatorname{ctx}_{\mathcal{N}}(u)\right\} \\
& \operatorname{Fn}_{\mathcal{G}}^{\mathcal{N}}\left(\left\langle X_{i}\right\rangle_{i \in[0, n]}\right) \stackrel{\text { def }}{=}\left\langle\left\lfloor\left\{X_{j} \circ X_{k} \mid X_{i} \rightarrow X_{j} X_{k} \in P\right\}\right\rfloor\right\rangle_{i \in[0, n]}
\end{aligned}
$$

where $X \circ Y \stackrel{\text { def }}{=}\left\{\left(q, q^{\prime}\right) \mid\left(q, q^{\prime \prime}\right) \in X \wedge\left(q^{\prime \prime}, q^{\prime}\right) \in Y\right\}$ is standard composition of two relations $X, Y \subseteq Q \times Q$.

LEMMA 4.5.13. The following hold:

(a) $\left\langle\wp\left(\Sigma^{*}\right), \subseteq\right\rangle \underset{\alpha}{\stackrel{\gamma}{\leftrightarrows}}\left\langle\mathrm{AC}_{\langle\wp(Q \times Q), \subseteq\rangle}\right.$, $\rangle$ is a $G C$.

(b) $\gamma \circ \alpha=\rho_{\leqslant N}$

(c) $\operatorname{Fn}_{\mathcal{G}}^{\mathcal{N}}(\vec{X})=\alpha \circ \operatorname{Fn}_{\mathcal{G}} \circ \gamma(\vec{X})$ for all $\vec{X} \in \alpha\left(\wp\left(\Sigma^{*}\right)^{|\mathcal{V}|}\right)$

\section{Proof.}

(a) Let us first observe that $\alpha$ and $\gamma$ are well-defined. First, $\alpha(X)$ is an antichain of $\langle\wp(Q \times Q), \subseteq\rangle$ since it is a minor for the well-quasiorder $\subseteq$ and, therefore, it is finite. On the other hand, $\gamma(Y)$ is clearly an element of $\left\langle\wp\left(\Sigma^{*}\right), \subseteq\right\rangle$ by definition.

Then, for all $X \in \wp\left(\Sigma^{*}\right)$ and $Y \in \mathrm{AC}_{\langle\wp(Q \times Q), \subseteq\rangle}$, it turns out that:

$$
\begin{aligned}
\alpha(X) \subseteq Y \Leftrightarrow & & {[\text { By definition of } \subseteq] } \\
\forall z \in \alpha(X), \exists y \in Y, y \subseteq z \Leftrightarrow & & {[\text { By definition of } \alpha \text { and }\lfloor\cdot]] } \\
\forall v \in X, \exists y \in Y, y \subseteq \operatorname{ctx}_{\mathcal{N}}(v) \Leftrightarrow & & {[\text { By definition of } \gamma] } \\
\forall v \in X, x \in \gamma(Y) \Leftrightarrow & & {[\text { By definition of } \subseteq] } \\
X \subseteq \gamma(Y) & &
\end{aligned}
$$


(b) For all $X \in \wp\left(\Sigma^{*}\right)$ we have that:

$$
\begin{array}{rlrl}
\gamma(\alpha(X)) & =\quad[\text { By definition of } \alpha, \gamma] \\
\left\{v \in \Sigma^{*} \mid \exists u \in \Sigma^{*}, \operatorname{ctx}_{\mathcal{N}}(u) \in\left\lfloor\left\{\operatorname{ctx}_{\mathcal{N}}(w) \mid w \in X\right\}\right\rfloor \wedge \operatorname{ctx} \mathcal{N}(u) \subseteq \operatorname{ctx} \mathcal{N}(v)\right\} & =\quad[\text { By definition of minor] } \\
\left\{v \in \Sigma^{*} \mid \exists u \in X, \operatorname{ctx}_{\mathcal{N}}(u) \subseteq \operatorname{ctx}_{\mathcal{N}}(v)\right\}= & \text { [By definition of } \left.\leqslant_{\mathcal{N}}\right] \\
\left\{v \in \Sigma^{*} \mid \exists u \in X, u \leqslant_{\mathcal{N}} v\right\} & =\quad\left[\text { By definition of } \rho_{\leqslant_{\mathcal{N}}}\right] \\
\rho_{\leqslant_{\mathcal{N}}}(X) & &
\end{array}
$$

(c) First, we show that $\operatorname{ctx}_{\mathcal{N}}(u v)=\operatorname{ctx}_{\mathcal{N}}(u) \circ \operatorname{ctx} \mathcal{N}_{\mathcal{N}}(v)$ for every pair of words $u, v \in \Sigma^{*}$.

$$
\begin{gathered}
\operatorname{ctx}_{\mathcal{N}}(u v)=[\text { By def. of } \operatorname{ctx} \mathcal{N}] \\
\left\{\left(q, q^{\prime}\right) \in Q^{2} \mid q \stackrel{u v}{\sim} q^{\prime}\right\}= \\
{\left[\text { Since } q \stackrel{u v}{\sim} q^{\prime} \Leftrightarrow \exists q^{\prime \prime} \in Q, q \stackrel{u}{\sim} q^{\prime \prime} \wedge q^{\prime \prime} \stackrel{v}{\sim} q^{\prime}\right]} \\
\left\{\left(q, q^{\prime}\right) \in Q^{2} \mid \exists q^{\prime \prime} \in Q, q \stackrel{u}{\sim} q^{\prime \prime} \wedge q^{\prime \prime} \stackrel{v}{\sim} q^{\prime}\right\}=
\end{gathered}
$$

[By definition of $\circ$ for binary relations]$$
\left\{\left(q, q^{\prime \prime}\right) \in Q^{2} \mid q \stackrel{u}{\sim} q^{\prime \prime}\right\} \circ\left\{\left(q^{\prime \prime}, q^{\prime}\right) \in Q^{2} \mid q^{\prime \prime} \stackrel{v}{\sim} q^{\prime}\right\}=
$$$$
\text { [By definition of } W_{q, q^{\prime}} \text { and ctx } \mathcal{N} \text { ] }
$$$$
\operatorname{ctx}_{\mathcal{N}}(u) \circ \operatorname{ctx}_{\mathcal{N}}(v)
$$

Secondly, we show that $\lfloor X \circ Y\rfloor=\lfloor\lfloor X\rfloor \circ\lfloor Y\rfloor\rfloor$ for every $X, Y \in \wp(Q \times Q)$. It is straightforward to check that $\lfloor X\rfloor \circ\lfloor Y\rfloor \subseteq X \circ Y$ and, therefore, $\lfloor\lfloor X\rfloor \circ\lfloor Y\rfloor\rfloor \subseteq\lfloor X \circ Y\rfloor$. Next, we prove the reverse inclusion by contradiction.

Let $x \circ y \in\lfloor X \circ Y\rfloor$ with $x \in X$ and $y \in Y$. Assume $x \circ y \notin\lfloor\lfloor\rfloor \circ\lfloor Y\rfloor\rfloor$. Then, there exists $\tilde{x} \in\lfloor X\rfloor$ and $\tilde{y} \in\lfloor Y\rfloor$ such that $\tilde{x} \circ \tilde{y} \in\lfloor\lfloor X\rfloor \circ\lfloor Y\rfloor\rfloor$ and $\tilde{x} \circ \tilde{y} \subseteq x \circ y$ which contradicts the fact that $x \circ y \in\lfloor X \circ Y\rfloor$ unless $\tilde{x} \circ \tilde{y}=x \circ y$, in which case $x \circ y \in\lfloor\lfloor X\rfloor \circ\lfloor Y\rfloor\rfloor$. Therefore, $\lfloor X \circ Y\rfloor \subseteq\lfloor\lfloor X\rfloor \circ\lfloor Y\rfloor\rfloor$.

Finally, we show that $\alpha\left(\operatorname{Fn}_{\mathcal{G}}(\gamma(\vec{X}))\right)=\operatorname{Fn}_{\mathcal{G}}^{\mathcal{N}}(\vec{X})$ for all $\vec{X} \in \alpha\left(\wp\left(\Sigma^{*}\right)\right)^{|\mathcal{V}|}$.

$$
\begin{array}{r}
\alpha\left(\operatorname{Fn}_{\mathcal{G}}(\gamma(\vec{X}))\right)= \\
{\left[\text { By definition of } \operatorname{Fn}_{\mathcal{G}}\right]} \\
\left\langle\alpha\left(\cup_{X_{i} \rightarrow X_{j} X_{k} \in P} \gamma\left(\vec{X}_{j}\right) \gamma\left(\vec{X}_{k}\right)\right)\right\rangle_{i \in[0, n]}=
\end{array}
$$




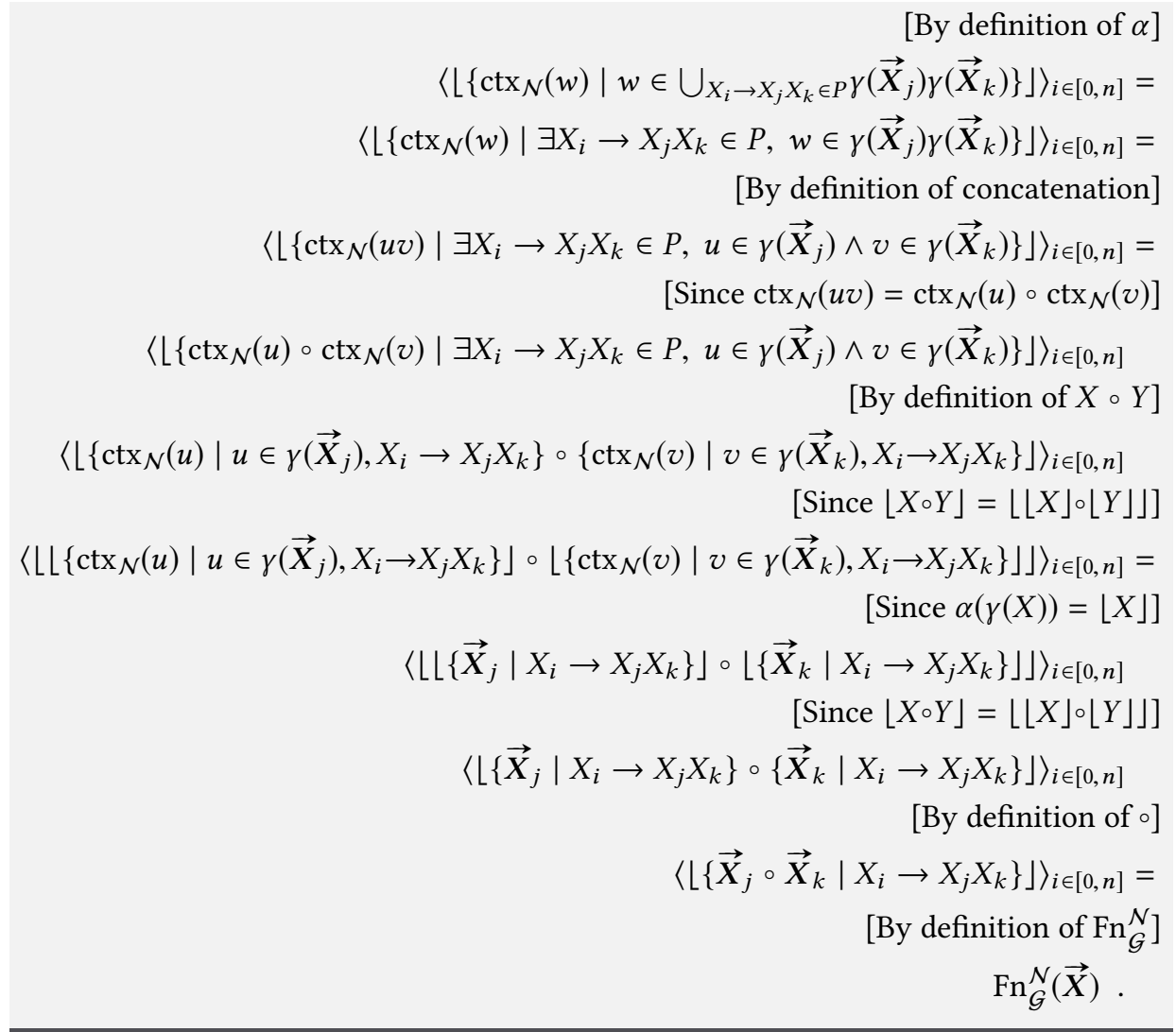

CFGINcS: State-based algorithm for $L(\mathcal{G}) \subseteq L(\mathcal{N})$

Data: $\mathrm{CFG} \mathcal{G}=\langle\mathcal{V}, \Sigma, P\rangle$ and NFA $\mathcal{N}=\langle Q, \Sigma, \delta, I, F\rangle$

$\mathbf{1}\left\langle Y_{i}\right\rangle_{i \in[0, n]}:=\operatorname{KLEENE}\left(\lambda \vec{X} \cdot\lfloor\vec{b}\rfloor \sqcup \operatorname{Fn}_{\mathcal{G}}^{\mathcal{N}}(\vec{X}), \vec{\varnothing}\right) ;$

2 forall $y \in Y_{0}$ do

3 if $y \cap(I \times F)=\varnothing$ then return false;

4 return true;

THEOREM 4.5.14. Let $\mathcal{G}$ be a CFG and $\mathcal{N}$ be an NFA. The algorithm CFGINCS decides $L(\mathcal{G}) \subseteq L(\mathcal{N})$.

Proof. We show that all the hypotheses (i)-(v) of Theorem 4.5.6 are satisfied for the abstract domain $\left\langle D, \leq_{D}\right\rangle=\left\langle\mathrm{AC}_{\langle\wp(Q \times Q), \subseteq\rangle}, \sqsubseteq\right\rangle$ as defined by the GC of Lemma 4.5.13 (a). 
(i) Since, by Lemma 4.5.13 (b), we have that $\rho_{\leqslant \mathcal{N}}(X)=\gamma(\alpha(X))$, it follows from Lemmas 4.5.7 (a) and 4.5.11 that $\gamma\left(\alpha\left(L_{2}\right)\right)=L_{2}$. Moreover, for every $a \in \sum$ and $X \in \wp\left(\Sigma^{*}\right)$ we have $\gamma \alpha(a X)=\gamma \alpha($ a $\alpha \alpha(X))$ :

$$
\begin{aligned}
\gamma \alpha(a X)= & {[\text { In GCs } \gamma=\gamma \alpha \gamma] } \\
\gamma \alpha \gamma \alpha(a X)= & {\left[\text { By Lemma } 4.5 .7\left(\text { b) with } \rho_{\leqslant_{N}}=\gamma \alpha\right]\right.} \\
\gamma \alpha \gamma \alpha(a \gamma \alpha(X))= & {[\text { In GCs } \gamma=\gamma \alpha \gamma] } \\
\gamma \alpha(\operatorname{ar} \alpha(X)) . &
\end{aligned}
$$

(ii) $\left(\mathrm{AC}_{\langle\wp(Q \times Q), \subseteq\rangle}\right.$, $)$ is effective because $Q$ is finite.

(iii) By Lemma 4.5.13 (c) we have that $\alpha\left(\operatorname{Fn}_{\mathcal{G}}(\gamma(\vec{X}))\right)=\operatorname{Fn}_{\mathcal{G}}^{\mathcal{N}}(\vec{X})$ for all vectors $\vec{X} \in \alpha\left(\wp\left(\Sigma^{*}\right)\right)^{|\mathcal{V}|}$.

(iv) $\alpha(\{b\})=\left\{\left(q, q^{\prime}\right) \mid q \stackrel{b}{\rightarrow} q^{\prime}\right\}$ and $\alpha(\varnothing)=\varnothing$, hence $\lfloor\alpha(\vec{b})\rfloor$ is trivial to compute.

(v) Since $\alpha\left(\overrightarrow{\boldsymbol{L}_{2}} X_{0}\right)=\left\langle\alpha\left(\psi_{\Sigma^{*}}^{L_{2}}(i=0)\right)\right\rangle_{i \in[0, n]}$, for all $\vec{Y} \in \alpha\left(\wp\left(\Sigma^{*}\right)\right)^{|\mathcal{V}|}$ the relation $\vec{Y} \sqsubseteq \alpha\left(\overrightarrow{\boldsymbol{L}_{2}} X_{0}\right)$ trivially holds for all components $Y_{i}$ with $i \neq 0$. For $Y_{0}$, it suffices to show that $Y_{0} \sqsubseteq \alpha\left(L_{2}\right) \Leftrightarrow \forall S \in Y_{q}, S \cap(I \times F) \neq \varnothing$, which is the check performed by lines 2-5 of algorithm CFGINCS.

$$
\begin{aligned}
Y_{0} \sqsubseteq \alpha\left(L_{2}\right) & \Leftrightarrow\left[\text { Since } Y_{0}=\alpha(U) \text { for some } U \in \wp\left(\Sigma^{*}\right)\right] \\
\alpha(U) \sqsubseteq \alpha\left(L_{2}\right) & \Leftrightarrow[\text { By GC] } \\
U \subseteq \gamma\left(\alpha\left(L_{2}\right)\right) & \Leftrightarrow
\end{aligned}
$$

[By Lemmas 4.5.7, 4.5.11 and 4.5.13, $\gamma\left(\alpha\left(L_{2}\right)\right)=L_{2}$ ]$$
U \subseteq L_{2} \Leftrightarrow\left[\text { Since } Y_{0}=\alpha(U)=\left\lfloor\left\{\operatorname{ctx}_{\mathcal{N}}(u) \mid u \in U\right\}\right\rfloor\right]
$$$$
\forall u \in U, \operatorname{ctx}_{\mathcal{N}}(u) \cap(I \times F) \neq \varnothing \Leftrightarrow \text { [By definition of } \operatorname{ctx}_{\mathcal{N}}(u) \text { ] }
$$$$
\forall S \in Y_{0}, S \cap I \neq \varnothing \text {. }
$$

Thus, by Theorem 4.5.6, Algorithm CFGINCS decides $\mathcal{L}(\mathcal{G}) \subseteq \mathcal{L}(\mathcal{N})$.

The resulting algorithm CFGINCS shares some features with two previous works. On the one hand, it is related to the work of Hofmann and Chen [2014] which defines an abstract interpretation-based language inclusion decision procedure similar to ours.

Even though Hofmann and Chen's algorithm and ours both manipulate sets of pairs of states of an automaton, their abstraction is based on equivalence relations and not quasiorders. Since quasiorders are strictly more general than equivalences our framework can be instantiated to a larger class of abstractions, most importantly coarser ones. Finally, it is worth pointing out that the approach of 
Hofmann and Chen [2014] aims at including languages of finite and also infinite words.

A second related work is that of Holík and Meyer [2015] who define an antichain like algorithm manipulating sets of pairs of states. Holík and Meyer [2015] tackle the language inclusion problem $\mathcal{L}(\mathcal{G}) \subseteq \mathcal{L}(\mathcal{N})$, where $\mathcal{G}$ is a grammar and $\mathcal{N}$ and automaton, by rephrasing the problem as a data flow analysis problem over a relational domain. In this scenario, the solution of the problem requires the computation of a least fixpoint on the relational domain, followed by an inclusion check between sets of relations. Then, they use the "antichains principle" to improve the performance of the fixpoint computation and, finally, they move from manipulating relations to manipulating pairs of states. As a consequence, Holík and Meyer [2015] obtain an antichains algorithm for checking the inclusion $\mathcal{L}(\mathcal{G}) \subseteq \mathcal{L}(\mathcal{N})$.

By contrast, our approach is direct and systematic, since we derive CFGINCS starting from the well-known Myhill quasiorder. We believe our approach evidences the relationship between the original antichains algorithm of Wulf et al. [2006] for regular languages and the one of Holík and Meyer [2015] for contextfree languages, which is the relation between Algorithms FAINCS and CFGINCS. Specifically, we show that these two algorithms are conceptually identical and differ in the quasiorder used to define the abstraction in which the computation takes place.

\subsection{An Equivalent Greatest Fixpoint Algorithm}

Let us recall from Cousot [2000, Theorem 4] that if $g: C \rightarrow C$ is a monotone function on a complete lattice $\langle C, \leq, \vee, \wedge\rangle$ which admits its unique right-adjoint $\widetilde{g}: C \rightarrow C$, i.e. $\forall c, c^{\prime} \in C, g(c) \leq c^{\prime} \Leftrightarrow c \leq \widetilde{g}\left(c^{\prime}\right)$ holds, then the following equivalence holds for all $c, c^{\prime} \in C$

$$
\operatorname{lfp}(\lambda x . c \vee g(x)) \leq c^{\prime} \Leftrightarrow c \leq \operatorname{gfp}\left(\lambda y . c^{\prime} \wedge \widetilde{g}(y)\right) .
$$

This property has been used by Cousot [2000] to derive equivalent least/greatest fixpoint-based invariance proof methods for programs.

In the following, we use Equivalence (4.25) to derive an algorithm for deciding the language inclusion $\mathcal{L}\left(\mathcal{N}_{1}\right) \subseteq \mathcal{L}\left(\mathcal{N}_{2}\right)$, which relies on the computation of a greatest fixpoint rather than a least fixpoint. This can be achieved by exploiting the following simple observation, which provides an adjunction between concatenation and quotients of sets of words.

LEMMA 4.6.1. For all $X, Y \in \wp\left(\Sigma^{*}\right)$ and $w \in \Sigma^{*}, w Y \subseteq Z \Leftrightarrow Y \subseteq w^{-1} Z$ and $Y w \subseteq Z \Leftrightarrow Y \subseteq Z w^{-1}$. 
Proof. By definition, for all $u \in \Sigma^{*}, u \in w^{-1} Z$ iff $w u \in Z$. Hence,

$$
Y \subseteq w^{-1} Z \Leftrightarrow \forall u \in Y, w u \in Z \Leftrightarrow w Y \subseteq Z
$$

Symmetrically, $Y w \subseteq Z \Leftrightarrow Y \subseteq Z w^{-1}$ holds.

Given an NFA $\mathcal{N}=\langle Q, \Sigma, \delta, I, F\rangle$, we define $\widetilde{\operatorname{Pre}}_{\mathcal{N}}: \wp\left(\Sigma^{*}\right)^{|Q|} \rightarrow \wp\left(\Sigma^{*}\right)^{|Q|}$ as a function on $Q$-indexed vectors of sets of words as follows:

$$
\widetilde{\operatorname{Pre}}_{\mathcal{N}}\left(\left\langle X_{q}\right\rangle_{q \in Q}\right) \stackrel{\text { def }}{=}\left\langle\bigcap_{a \in \Sigma, q^{\prime} \in \delta(q, a)} a^{-1} X_{q}\right\rangle_{q^{\prime} \in Q}
$$

where, as usual, $\bigcap \varnothing=\Sigma^{*}$. It turns out that $\widetilde{\operatorname{Pre}}_{\mathcal{N}}$ is the usual weakest liberal precondition which is right-adjoint of $\operatorname{Pre}_{\mathcal{N}}$.

LemмA 4.6.2. For all $\vec{X}, \vec{Y} \in \wp\left(\Sigma^{*}\right)^{|Q|}, \operatorname{Pre}_{\mathcal{N}}(\vec{X}) \subseteq \vec{Y} \Leftrightarrow \vec{X} \subseteq \widetilde{\operatorname{Pre}}_{\mathcal{N}}(\vec{Y})$.

Proof. For all $\vec{X}, \vec{Y} \in \wp\left(\Sigma^{*}\right)^{|Q|}$,

$$
\begin{array}{rll}
\operatorname{Pre}_{\mathcal{N}}\left(\left\langle X_{q}\right\rangle_{q \in Q}\right) \subseteq\left\langle Y_{q}\right\rangle_{q \in Q} \Leftrightarrow & {[\text { By definition of Pre }} \\
\left.\forall q \in Q, \bigcup_{q \rightarrow q^{\prime}}\right] \\
\forall X_{q^{\prime}} \subseteq Y_{q} \Leftrightarrow & \\
\forall q, q^{\prime} \in Q, q \stackrel{a}{\rightarrow} q^{\prime} \Rightarrow a X_{q^{\prime}} \subseteq Y_{q} \Leftrightarrow & {[\text { By Lemma 4.6.1] }} \\
\forall q, q^{\prime} \in Q, q \stackrel{a}{\rightarrow} q^{\prime} \Rightarrow X_{q^{\prime}} \subseteq a^{-1} Y_{q} \Leftrightarrow & {\left[\left(\forall i \in I, X \subseteq Y_{i}\right) \Leftrightarrow X \subseteq \cap_{i \in I} Y_{i}\right]} \\
\forall q^{\prime} \in Q, X_{q^{\prime}} \subseteq \bigcap_{q \rightarrow q^{\prime}} a^{-1} Y_{q} \Leftrightarrow & {\left[\text { By definition of } \widetilde{\operatorname{Pre}}_{\mathcal{N}}\right]} \\
\left\langle X_{q}\right\rangle_{q \in Q} \subseteq \widetilde{\operatorname{Pre}}_{\mathcal{N}}\left(\left\langle Y_{q}\right\rangle_{q \in Q}\right) &
\end{array}
$$

Hence, from Equivalences (4.6) and (4.25) we obtain:

$$
\mathcal{L}\left(\mathcal{N}_{1}\right) \subseteq L_{2} \Leftrightarrow \overrightarrow{\boldsymbol{\epsilon}} F^{F_{1}} \subseteq \operatorname{gfp}\left(\lambda \vec{X} \cdot{\overrightarrow{L_{2}}}_{I_{1}} \cap \widetilde{\operatorname{Pre}}_{\mathcal{N}_{1}}(\vec{X})\right)
$$

The following algorithm FAINCGFP decides the inclusion $\mathcal{L}\left(\mathcal{N}_{1}\right) \subseteq L_{2}$ by implementing the greatest fixpoint computation from Equivalence (4.26). The intuition behind algorithm FAINCGFP is that

$$
\mathcal{L}\left(\mathcal{N}_{1}\right) \subseteq L_{2} \Leftrightarrow \epsilon \in \bigcap_{w \in \mathcal{L}\left(\mathcal{N}_{1}\right)^{w^{-1}} L_{2}}
$$

Therefore, FAIncGFP computes the set $\bigcap_{w \in \mathcal{L}\left(\mathcal{N}_{1}\right)} w^{-1} L_{2}$ by using the automaton $\mathcal{N}_{1}$ and by considering prefixes of $\mathcal{L}\left(\mathcal{N}_{1}\right)$ of increasing lengths. This means that 
FAINCGFP: Greatest fixpoint algorithm for $\mathcal{L}\left(\mathcal{N}_{1}\right) \subseteq L_{2}$

Data: NFA $\mathcal{N}_{1}=\left\langle Q_{1}, \delta_{1}, I_{1}, F_{1}, \Sigma\right\rangle$; regular language $L_{2}$.

$1\left\langle Y_{q}\right\rangle_{q \in Q}:=\operatorname{KLEENE}\left(\lambda \vec{X} \cdot{\overrightarrow{L_{2}}}_{I_{1}} \cap \widetilde{\operatorname{Pre}}_{\mathcal{N}_{1}}(\vec{X}), \overrightarrow{\Sigma^{*}}\right)$;

2 forall $q \in F_{1}$ do

3 if $\epsilon \notin Y_{q}$ then return false;

4 return true;

after $n$ iterations of the KLEENE procedure, Algorithm FAIncGFp has computed, for every state $q \in Q_{1}$, the set

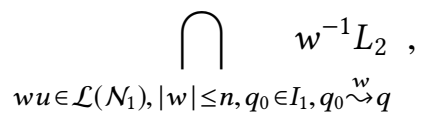

The regularity of $L_{2}$ together with the property of regular languages of being closed under intersections and quotients show that each iterate computed by $\operatorname{KLEENE}\left(\lambda \vec{X} \cdot{\overrightarrow{L_{2}}}^{I_{1}} \cap \overrightarrow{\operatorname{Pre}}_{\mathcal{N}_{1}}(\vec{X}), \overrightarrow{\Sigma^{*}}\right)$ is a (computable) regular language. To the best of our knowledge, this language inclusion algorithm FAINCGFP has never been described in the literature before.

Next, we discharge the fundamental assumption on which the correctness of this algorithm FAINCGFP depends on: the Kleene iterates computed by FAINCGFP are finitely many. In order to do that, we consider an abstract version of the greatest fixpoint computation exploiting a closure operator which guarantees that the abstract Kleene iterates are finitely many. This closure operator $\rho_{\leqslant_{N_{2}}}$ will be defined by using an ordering relation $\leqslant_{N_{2}}$ induced by an NFA $\mathcal{N}_{2}$ such that $L_{2}=\mathcal{L}\left(\mathcal{N}_{2}\right)$ and will be shown to be forward complete for the function $\lambda \vec{X} \cdot{\overrightarrow{L_{2}}}_{I_{1}} \cap \widetilde{\operatorname{Pre}_{\mathcal{N}_{1}}}(\vec{X})$ used by FAIncGFP.

Forward completeness of abstract interpretations [Giacobazzi and Quintarelli 2001], also called exactness [Miné 2017, Definition 2.15], is different from and orthogonal to backward completeness introduced in Section 4.1 and crucially used in Sections 4.2-4.5. In particular, a remarkable consequence of exploiting a forward complete abstraction is that the Kleene iterates of the concrete and abstract greatest fixpoint computations coincide.

The intuition here is that this forward complete closure $\rho_{\leq_{N_{2}}}$ allows us to establish that all Kleene iterates of $\operatorname{gfp}\left(\vec{X} \cdot{\overrightarrow{L_{2}}}_{I_{1}} \cap \widetilde{\operatorname{Pre}}_{\mathcal{N}_{1}}(\vec{X})\right)$ belong to the image of the closure $\rho_{\leqslant_{N_{2}}}$. More precisely, every Kleene iterate is a language which is upward closed for $\leqslant_{\mathcal{N}_{2}}$. Interestingly, a similar phenomenon occurs in wellstructured transition systems [Abdulla et al. 1996; Finkel and Schnoebelen 2001].

Let us now describe in detail this abstraction. A closure $\rho \in \operatorname{uco}(C)$ on a 
concrete domain $C$ is forward complete for a monotone function $f: C \rightarrow C$ iff $\rho f \rho=f \rho$ holds. The intuition here is that forward completeness means that no loss of precision is accumulated when the output of a computation of $f \rho$ is approximated by $\rho$, or, equivalently, $f$ maps abstract elements of $\rho$ into abstract elements of $\rho$. Dually to the case of backward completeness, forward completeness implies that $\operatorname{gfp}(f)=\operatorname{gfp}(f \rho)=\operatorname{gfp}(\rho f \rho)$, when these greatest fixpoints exist (this is the case, e.g., when $C$ is a complete lattice).

It turns out that forward and backward completeness are related by the following duality on function $f$.

LEMMA 4.6.3 ([Giacobazzi and Quintarelli 2001, Corollary 1]). Let $\langle C, \leq\rangle$ be a complete lattice and assume that $f: C \rightarrow C$ admits the right-adjoint $\widetilde{f}: C \rightarrow C$, i.e. $f(c) \leq c^{\prime} \Leftrightarrow c \leqq \widetilde{f}\left(c^{\prime}\right)$ holds. Then, $\rho$ is backward complete for $f$ iff $\rho$ is forward complete for $\widetilde{f}$.

Thus, by Lemma 4.6.3, in the following result instead of assuming the hypotheses implying that a closure $\rho$ is forward complete for the right-adjoint $\operatorname{Pre}_{\mathcal{N}_{1}}$ we state some hypotheses which guarantee that $\rho$ is backward complete for its left-adjoint, which, by Lemma 4.6.2, is $\operatorname{Pre}_{\mathcal{N}_{1}}$.

THeOREM 4.6.4. Let $\mathcal{N}_{1}=\left\langle Q_{1}, \delta_{1}, I_{1}, F_{1}, \Sigma\right\rangle$ be an $N F A$, let $L_{2}$ be a regular language and let $\rho \in \operatorname{uco}\left(\left\langle\wp\left(\Sigma^{*}\right), \subseteq\right\rangle\right)$. Let us assume that:

(a) $\rho\left(L_{2}\right)=L_{2}$;

(b) $\rho$ is backward complete for $\lambda X$. aX for all $a \in \Sigma$.

Then

$$
\mathcal{L}\left(\mathcal{N}_{1}\right) \subseteq L_{2} \Leftrightarrow \overrightarrow{\boldsymbol{\epsilon}}^{F_{1}} \subseteq \operatorname{gfp}\left(\overrightarrow{\boldsymbol{X}} \cdot \rho\left(\overrightarrow{\boldsymbol{L}}_{2}^{I_{1}} \cap \widetilde{\operatorname{Pre}}_{\mathcal{N}_{1}}(\rho(\overrightarrow{\boldsymbol{X}}))\right)\right) .
$$

Moreover, the Kleene iterates computed by $\operatorname{gfp}\left(\overrightarrow{\boldsymbol{X}} \cdot \rho\left({\overrightarrow{\boldsymbol{L}_{2}}}_{I_{1}} \cap \widetilde{\operatorname{Pre}}_{\mathcal{N}_{1}}(\rho(\vec{X}))\right)\right)$ coincide in lockstep with those of $\operatorname{gfp}\left(\vec{X} \cdot \vec{L}_{2}^{I_{1}} \cap \widetilde{\operatorname{Pre}}_{\mathcal{N}_{1}}(\vec{X})\right)$.

Proof. Theorem 4.2.3 shows that if $\rho$ is backward complete for $\lambda X . a X$ for every $a \in \Sigma$ then it is backward complete for Pre $\mathcal{N}_{1}$. Thus, by Lemma 4.6.3, $\rho$ is forward complete for $\widetilde{\operatorname{Pre}}_{\mathcal{N}_{1}}$, hence it is forward complete for $\lambda \vec{X} \cdot \overrightarrow{L_{2}^{I_{1}}} \cap \widetilde{\operatorname{Pre}}_{\mathcal{N}_{1}}(\vec{X})$ since:

$$
\begin{aligned}
\rho\left(\overrightarrow{\boldsymbol{L}}_{2}^{I_{1}} \cap \widetilde{\operatorname{Pre}}_{\mathcal{N}_{1}}(\rho(\overrightarrow{\boldsymbol{X}}))\right)= & {\left[\text { By forward comp. for } \widetilde{\operatorname{Pre}}_{\mathcal{N}_{1}} \text { and } \rho\left(L_{2}\right)=L_{2}\right] } \\
\rho\left(\rho\left(\overrightarrow{\boldsymbol{L}}_{2} I_{1}\right) \cap \rho\left(\widetilde{\operatorname{Pre}}_{\mathcal{N}_{1}}(\rho(\overrightarrow{\boldsymbol{X}}))\right)\right)= & {[\text { Since } \rho(\cap \rho(X))=\cap \rho(X)] } \\
\rho\left(\overrightarrow{\boldsymbol{L}}_{2}^{I_{1}}\right) \cap \rho\left(\widetilde{\operatorname{Pre}}_{\mathcal{N}_{1}}(\rho(\overrightarrow{\boldsymbol{X}}))\right)= & {\left[\text { By forward comp. for } \widetilde{\operatorname{Pre}}_{\mathcal{N}_{1}} \text { and } \rho\left(L_{2}\right)=L_{2}\right] } \\
{\overrightarrow{\boldsymbol{L}_{2}}}_{I_{1}} \cap \widetilde{\operatorname{Pre}}_{\mathcal{N}_{1}}(\rho(\overrightarrow{\boldsymbol{X}})) &
\end{aligned}
$$


Since, by forward completeness, we have that

$$
\operatorname{gfp}\left(\overrightarrow{\boldsymbol{X}} \cdot{\overrightarrow{\boldsymbol{L}_{2}}}_{I_{1}}^{I_{1}} \cap \widetilde{\operatorname{Pre}}_{\mathcal{N}_{1}}(\overrightarrow{\boldsymbol{X}})\right)=\operatorname{gfp}\left(\overrightarrow{\boldsymbol{X}} \cdot \rho\left({\overrightarrow{\boldsymbol{L}_{2}}}_{I_{1}}^{I_{1}} \cap \widetilde{\operatorname{Pre}}_{\mathcal{N}_{1}}(\rho(\vec{X}))\right)\right),
$$

by Equivalence (4.26), we conclude that

$$
\mathcal{L}\left(\mathcal{N}_{1}\right) \subseteq L_{2} \Leftrightarrow \overrightarrow{\boldsymbol{\epsilon}}^{F_{1}} \subseteq \operatorname{gfp}\left(\vec{X} \cdot \rho\left({\overrightarrow{L_{2}}}^{I_{1}} \cap \widetilde{\operatorname{Pre}}_{\mathcal{N}_{1}}(\rho(\vec{X}))\right)\right)
$$

Finally, we observe that the Kleene iterates computing $\operatorname{gfp}\left(\lambda \vec{X} \cdot{\overrightarrow{L_{2}}}_{I_{1}} \cap \widetilde{\operatorname{Pre}_{\mathcal{N}_{1}}}(\vec{X})\right)$ and those computing $\operatorname{gfp}\left(\overrightarrow{\boldsymbol{X}} \cdot \rho\left({\overrightarrow{\boldsymbol{L}_{2}}}_{I_{1}}^{I_{1}} \cap \widetilde{\operatorname{Pre}}_{\mathcal{N}_{1}}(\rho(\overrightarrow{\boldsymbol{X}}))\right)\right)$ coincide in lockstep since $\rho\left(\overrightarrow{\boldsymbol{L}}_{2}^{I_{1}} \cap \widetilde{\operatorname{Pre}}_{\mathcal{N}_{1}}(\rho(\overrightarrow{\boldsymbol{X}}))\right)={\overrightarrow{\boldsymbol{L}_{2}}}^{I_{1}} \cap \overrightarrow{\operatorname{Pre}}_{\mathcal{N}_{1}}(\rho(\overrightarrow{\boldsymbol{X}}))$ and $\rho\left(\overrightarrow{\boldsymbol{L}}_{2}^{I_{1}}\right)={\overrightarrow{\boldsymbol{L}_{2}}}_{I_{1}}$.

We can now establish that the sequence of Kleene iterates computed by $\operatorname{gfp}\left(\vec{X} \cdot \vec{L}_{2}^{I_{1}} \cap \widehat{\operatorname{Pre}}_{\mathcal{N}_{1}}(\vec{X})\right)$ is finite. Let $L_{2}=\mathcal{L}\left(\mathcal{N}_{2}\right)$, for some NFA $\mathcal{N}_{2}$, and consider the corresponding left state-based quasiorder $\leqslant_{N_{2}}^{l}$ on $\Sigma^{*}$ as defined by (4.12).

Lemma 4.3.7 tells us that $\leqslant_{\mathcal{N}_{2}}^{l}$ is a left $L_{2}$-consistent wqo. Furthermore, since $Q_{2}$ is finite we have that both $\leqslant_{N_{2}}^{l}$ and $\left(\leqslant_{N_{2}}^{l}\right)^{-1}$ are wqos, so that, in turn, $\left\langle\rho_{\leqslant_{N_{2}}^{l}}, \subseteq\right\rangle$ is a poset which is both ACC and DCC. In particular, the definition of $\leqslant_{N_{2}}^{l}$ implies that every chain in $\left\langle\rho_{\leqslant_{N_{2}}}, \subseteq\right\rangle$ has at most $2^{\left|Q_{2}\right|}$ elements, so that if we compute $2^{\left|Q_{2}\right|}$ Kleene iterates then we have surely computed the greatest fixpoint.

Moreover, as a consequence of the DCC we have that the Kleene iterates of $\operatorname{gfp}\left(\lambda \vec{X} \cdot \rho_{\leq_{N_{2}}}\left(\overrightarrow{\boldsymbol{L}}_{2}^{I_{1}} \cap \widetilde{\operatorname{Pre}}_{\mathcal{N}_{1}}\left(\rho_{\leq_{\mathcal{N}_{2}}}(\vec{X})\right)\right)\right)$ are finitely many, hence so are the iterates of $\operatorname{gfp}\left(\lambda \vec{X} \cdot{\overrightarrow{L_{2}}}^{I_{1}} \cap \widetilde{\operatorname{Pre}}_{\mathcal{N}_{1}}(\vec{X})\right)$ because they go in lockstep as stated by Theorem 4.6.4.

COROLLARY 4.6.5. Let $\mathcal{N}_{1}$ be an NFA and let $L_{2}$ be a regular language. Then, the algorithm FAINCGFP decides the inclusion $\mathcal{L}\left(\mathcal{N}_{1}\right) \subseteq L_{2}$

Finally, it is worth citing that Fiedor et al. [2015] put forward an algorithm for deciding WS1S formula which relies on the same lfp computation used in FAINcS. Then, they derive a dual gfp computation by relying on Park's duality [Park 1969]: $\operatorname{lfp}(\lambda X . f(X))=\left(\operatorname{gfp}\left(\lambda X .\left(f\left(X^{c}\right)\right)^{c}\right)\right)^{c}$. Their approach differs from ours since we use the Equivalence (4.25) to compute a gfp, different from the lfp, which still allows us to decide the inclusion problem. Furthermore, their algorithm decides whether a given automaton accepts $\epsilon$ and it is not clear how their algorithm could be extended for deciding language inclusion. 


\section{SeArching on Compressed TeXt}

In this chapter, we show how to instantiate the quasiorder-based framework presented in Chapter 4 to search on compressed text. Specifically, we adapt Algorithm CFGINCS to report the number of lines in a grammar-compressed text containing a match for a given regular expression.

The problem of searching in compressed text is of practical interest due the growing amount of information handled by modern systems, which demands efficient techniques both for compression, to reduce the storage cost, and for regular expression searching, to speed up querying. As an evidence of the importance of this problem, note that state of the art tools for searching with regular expressions, such as grep and ripgrep, provide a method to search on compressed files by decompressing them on-the-fly.

In the following, we focus on the problem of counting, i.e. finding the number of lines of the input text that contain a match for the expression. This type of query is supported out of the box by many tools ${ }^{1}$, which evidences its practical interest. However, when the text is given in compressed form, the fastest approach in practice is the decompress and search approach, i.e. querying the uncompressed text as it is recovered by the decompressor. In this chapter, we challenge this approach.

Lossless compression of textual data is achieved by finding repetitions in the input text and replacing them by references. We focus on grammar-based compression schemes in which each tuple "reference $\rightarrow$ repeated text" is considered as a rule of a context-free grammar. The resulting grammar, produced as the

\footnotetext{
${ }^{1}$ Tools such as grep, ripgrep, awk and ag, among others, can be used to report the number of matching lines in a text.
} 
output of the compression, generates a language consisting of a single word: the uncompressed text. Figure 5.1 depicts the output of a grammar-based compression algorithm.

$$
\begin{aligned}
& X_{6} \rightarrow X_{5} X_{5} \\
& X_{5} \rightarrow X_{3} X_{4} \\
& X_{4} \rightarrow X_{2} \mathrm{~b} \\
& X_{3} \rightarrow X_{1} \$ \\
& X_{2} \rightarrow \mathrm{a} \$ \\
& X_{1} \rightarrow \mathrm{ab}
\end{aligned}
$$

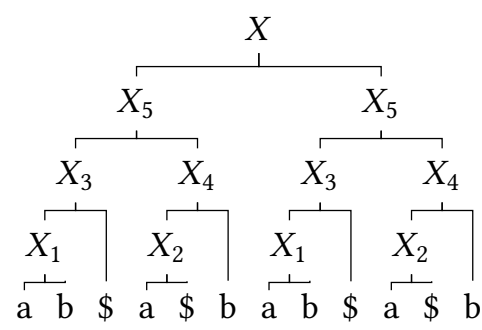

Figure 5.1: List of grammar rules (left) generating the string " $a b \$ a \$ b a b \$ a \$ b$ " (and no other) as evidenced by the parse tree (right).

Intuitively, the decompress and search approach prevents the searching algorithm from taking advantage of the repetitions in the data found by the compressor. For instance, in the grammar shown in Figure 5.1, the decompress and search approach results in processing the subsequence "ab\$a $\$ b$ " twice. By working on the compressed data, our algorithm would process that subsequence once and reuse the information each time it finds the variable $X_{5}$.

Given a grammar-compressed text and a regular expression, deciding whether the compressed text matches the expression amounts to deciding the emptiness of the intersection of the languages generated by the grammar and an automaton built for the regular expression.

In order to solve this emptiness problem, we reduce it to an inclusion problem. Note that this reduction is possible since the grammar generates a single word and, therefore, $\{w\} \cap L \neq \varnothing \Leftrightarrow w \in L$, where $L$ is the language generated by the regular expression. Then, we could instantiate the quasiorder-based framework described in Chapter 4 with different quasiorders to decide the inclusion.

However, in order to go beyond a yes/no answer and report or count the exact matches, we need to compute some extra information for each variable of the grammar. This extra information is computed for the terminals of the grammar and then propagated through the variables according to the grammar rules in a bottom-up fashion. To do that, we iterate thorough the grammar rules and compose, for each of them, the information previously computed for the variables on the right hand side. For example, when processing rule $X_{3} \rightarrow X_{1} \$$ of Figure 5.1 our algorithm composes the information for $X_{1}$ with the one for $\$$. The information computed for the string "ab\$", will be reused every time the variable $X_{3}$ appears in the right hand side of a rule. 
Following this idea, we present an algorithm for counting the lines in a grammar-compressed text containing a match for a regular expression whose runtime does not depend on the size $T$ of the uncompressed text. Instead, it runs in time linear in the size of its compressed version. Furthermore, the information computed for counting can be used to perform an on-the-fly, lazy decompression to recover the matching lines from the compressed text. Note that, for reporting the matching lines, the dependency on $T$ in unavoidable.

The salient features of our approach are:

\section{Generality}

Our algorithm is not tied to any particular grammar-based compressor. Instead, we consider the compressed text is given by a straight line program (SLP for short), i.e. a grammar generating the uncompressed text and nothing else.

Finding the smallest SLP $g$ generating a text of length $T$ is an NP-hard problem, as shown by Charikar et al. [2005], for which grammar-based compressors such as LZ78 [Ziv and Lempel 1978], LZW [Welch 1984], RePair [Larsson and Moffat 1999] and Sequitur [Nevill-Manning and Witten 1997] produce different approximations. For instance, Hucke et al. [2016] showed that the LZ78 algorithm produces a representation of size $\Omega\left(|g| \cdot(T / \log T)^{2 / 3}\right)$ and the representation produced by the RePair algorithm has size $\Omega(|g| \cdot \log T / \log (\log T))$.

Since it is defined over SLPs, our algorithm applies to all such approximations, including $g$ itself.

\section{Nearly optimal data structures}

We define data structures enabling the algorithm to run in time linear in the size of the compressed text. With these data structures our algorithm runs in $O\left(t \cdot s^{3}\right)$ time using $O\left(t \cdot s^{2}\right)$ space where $t$ is the size of the compressed text, i.e. the grammar, and $s$ is the size of the automaton built from the regular expression. When the automaton is deterministic, the complexity drops to $O(t \cdot s)$ time and $O(t \cdot s)$ space.

As shown by Abboud et al. [2017], there is no combinatorial ${ }^{2}$ algorithm improving these time complexity bounds beyond polylog factors, hence our algorithm is nearly optimal.

\footnotetext{
${ }^{2}$ Interpreted as any practically efficient algorithm that does not suffer from the issues of Fast Matrix Multiplication such as large constants and inefficient memory usage.
} 


\section{Efficient implementation}

We present zearch, a purely sequential implementation of our algorithm which uses the above mentioned data structures. ${ }^{3}$

The experiments show that zearch requires up to $25 \%$ less time than the state of the art: running hyperscan on the uncompressed text as it is recovered by $1 z 4$ (in parallel). Furthermore, when the grammar-based compressor achieves high compression ratio (above 13:1), running zearch on the compressed text is as fast as running hyperscan directly on the uncompressed text. Such compression ratios are achieved, for instance, when working with automatically generated $\log$ files.

\subsection{Finding the Matches}

Recall that the problem of deciding whether a grammar-compressed text contains a match for a regular expression can be reduced to an emptiness problem for the intersection of the languages generated by a grammar and an automaton. Indeed, given an SLP $\mathcal{P}$ generating a text $T$ over an alphabet $\Sigma$, i.e. $\mathcal{L}(\mathcal{P})=\{T\}$ where $T \in \Sigma^{*}$, and an automaton $\mathcal{N}=\langle Q, \Sigma, \delta, I, F\rangle$ representing a regular expression, we find that:

There exists a substring of $T$ in $\mathcal{L}(\mathcal{N}) \Leftrightarrow \mathcal{L}(\mathcal{P}) \cap\left(\Sigma^{*} \cdot \mathcal{L}(\mathcal{N}) \cdot \Sigma^{*}\right) \neq \varnothing$.

On the other hand, since $\mathcal{L}(\mathcal{P})$ contains exactly one word we have that

$$
\mathcal{L}(\mathcal{P}) \cap\left(\Sigma^{*} \cdot \mathcal{L}(\mathcal{N}) \cdot \Sigma^{*}\right) \neq \varnothing \Leftrightarrow \mathcal{L}(\mathcal{P}) \subseteq\left(\Sigma^{*} \cdot \mathcal{L}(\mathcal{N}) \cdot \Sigma^{*}\right)
$$

As a consequence, the problem of deciding whether a grammar-compressed text contains a match for a regular expression can be solved by using Algorithm CFGINCS with the quasiorder $\leqslant_{\mathcal{N}}$ as described in Chapter 4.

Observe that, as the following example evidences, when restricting to SLPs the iteration of the KLEENE procedure updates the abstraction for each variable of the grammar exactly once since there are no loops in SLPs. As a consequence, it is enough to process the rules in an orderly manner and compute the abstraction for each variable, i.e. $\alpha(X)$, exactly once.

Example 5.1.1. Let $\mathcal{P}$ be the SLP from Figure 5.1 and let $\mathcal{N}$ and $\mathcal{N}^{\prime}$ be the automata from Figure 5.2. Next, we show the Kleene iterates computed by Algorithm CFGINCS which, as shown in Chapter 4, works on the abstract domain $\left\langle\mathrm{AC}_{\langle\wp(Q \times Q), \subseteq\rangle}, \sqsubseteq\right\rangle$ with the abstraction function defined as $\alpha(X)=\left\lfloor\left\{\operatorname{ctx}_{\mathcal{N}}(u) \mid u \in X\right\}\right\rfloor$.

\footnotetext{
${ }^{3}$ zearch can optionally report the matching lines.
} 


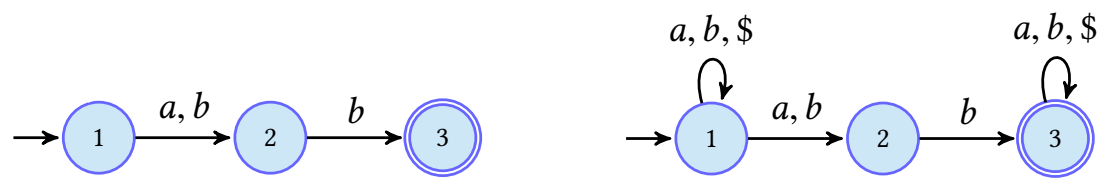

Figure 5.2: NFAs $\mathcal{N}^{\prime}$ (left) and $\mathcal{N}$ (right) on $\Sigma=\{a, b, \$\}$ with $\mathcal{L}\left(\mathcal{N}^{\prime}\right)=\{a b, b b\}$ and $\mathcal{L}(\mathcal{N})=\Sigma^{*} \cdot \mathcal{L}\left(\mathcal{N}^{\prime}\right) \cdot \Sigma^{*}$

To simplify the notation, we denote the pair $\left(q_{i}, q_{j}\right)$ by $i j$.

$\left(\begin{array}{c}\alpha\left(W_{X_{\mathscr{S}}}^{\mathcal{P}}\right) \\ \alpha\left(W_{X_{X}}^{\mathcal{P}}\right) \\ \alpha\left(W_{X_{X}}^{\mathcal{P}}\right) \\ \alpha\left(W_{X_{3}}^{\mathcal{P}}\right) \\ \alpha\left(W_{X_{2}}^{\mathcal{P}}\right) \\ \alpha\left(W_{X_{1}}^{\mathcal{P}}\right)\end{array}\right)=\left(\begin{array}{c}\varnothing \\ \varnothing \\ \varnothing \\ \varnothing \\ \lfloor\{11,33\}\rfloor \\ \lfloor\{11,33,13\}\rfloor\end{array}\right) \Rightarrow\left(\begin{array}{c}\varnothing \\ \varnothing \\ \lfloor\{11,33\}\rfloor \\ \lfloor\{11,33,13\}\rfloor \\ \lfloor\{11,33\}\rfloor \\ \lfloor\{11,33,13\}\rfloor\end{array}\right) \Rightarrow\left(\begin{array}{c}\varnothing \\ \lfloor\{11,33,13\}\rfloor \\ \lfloor\{11,33\}\rfloor \\ \lfloor\{11,33,13\}\rfloor \\ \lfloor\{11,33\}\rfloor \\ \lfloor\{11,33,13\}\rfloor\end{array}\right) \Rightarrow\left(\begin{array}{c}\lfloor\{11,33,13\}\rfloor \\ \lfloor\{11,33,13\}\rfloor \\ \lfloor\{11,33\}\rfloor \\ \lfloor\{11,33,13\}\rfloor \\ \lfloor\{11,33\}\rfloor \\ \lfloor\{11,33,13\}\rfloor\end{array}\right)$

Since for every variable $X_{n}$ the value of $\alpha\left(X_{n}\right)$ is computed by combining the values of $\alpha\left(X_{i}\right)$ and $\alpha\left(X_{j}\right)$ for some $i, j<n$, the KLEENE procedure is equivalent to computing, sequentially, the values of $\alpha\left(X_{1}\right), \alpha\left(X_{2}\right), \ldots, \alpha\left(X_{5}\right)$.

In this case, since $\left(q_{1}, q_{3}\right) \in \alpha\left(X_{5}\right)$ Algorithm CFGINCS concludes that the inclusion $\mathcal{L}(\mathcal{P}) \subseteq \mathcal{L}(\mathcal{N})$ holds, i.e. there exists a substring $w$ of the uncompressed such that $w \in \mathcal{L}\left(\mathcal{N}^{\prime}\right)$.

Furthermore, in an SLP each variable generates exactly one word and, therefore, the abstraction of a variable consists of a single set, i.e. $\alpha(X) \in \mathrm{AC}_{\langle\wp(Q \times Q), \subseteq\rangle}$ is a singleton as shown in Example 5.1.1. As a consequence, we can drop the $\lfloor\cdot\rfloor$ from function $\operatorname{Fn}_{\mathcal{P}}^{\mathcal{N}}$ defined in Section 4.5.5, since $\left\lfloor\left\{\operatorname{ctx}_{\mathcal{N}}(w)\right\}\right\rfloor=\left\{\operatorname{ctx}_{\mathcal{N}}(w)\right\}$ for any word, and write:

$$
\operatorname{Fn}_{\mathcal{P}}^{\mathcal{N}}\left(\left\langle X_{i}\right\rangle_{i \in[0, n]} \stackrel{\text { def }}{=}\left\langle\left\{X_{j} \circ X_{k} \mid X_{i} \rightarrow X_{j} X_{k} \in P\right\}\right\rangle_{i \in[0, n]}\right.
$$

Recall that, by definition, for all $X_{j}, X_{k} \in \wp(Q \times Q)^{|\mathcal{V}|}$,

$$
X_{j} \circ X_{k}=\left\{\left(q_{1}, q_{2}\right) \mid \exists q^{\prime} \in Q,\left(q_{1}, q^{\prime}\right) \in X_{j} \wedge\left(q^{\prime}, q_{2}\right) \in X_{k}\right\} .
$$

Finally, given an NFA $\mathcal{N}^{\prime}$ it is straightforward to build an automaton $\mathcal{N}$ generating the language $\Sigma^{*} \cdot \mathcal{L}(\mathcal{N}) \cdot \Sigma^{*}$ by adding self-loops reading each letter of the alphabet to every initial and every final state of $\mathcal{N}^{\prime}$ as shown in Figure 5.2. Instead of adding these transitions to $\mathcal{N}$, which, as shown in Example 5.1.1, results in adding the pairs $\{(q, q) \mid q \in I \cup F\}$ to $\operatorname{ctx}_{\mathcal{N}}(w)$ for every word $w \in \Sigma^{*}$, we consider them as implicit. 
As a consequence, when the input grammar is an SLP and we are interested in deciding whether $\mathcal{L}(\mathcal{P}) \subseteq \Sigma^{*} \cdot \mathcal{L}(\mathcal{N}) \cdot \Sigma^{*}$, Algorithm CFGINCS can be written as Algorithm SLPINCS. Observe that Algorithm SLPINCS uses the transition function $\delta$ to store and manipulate the sets $\operatorname{ctx}_{\mathcal{N}}\left(X_{i}\right)$ for each variable $X_{i}$ of the grammar, i.e.

$$
\left(q_{1}, X_{i}, q_{2}\right) \in \delta \Leftrightarrow\left(q_{1}, q_{2}\right) \in \operatorname{ctx}_{\mathcal{N}}\left(X_{i}\right)
$$

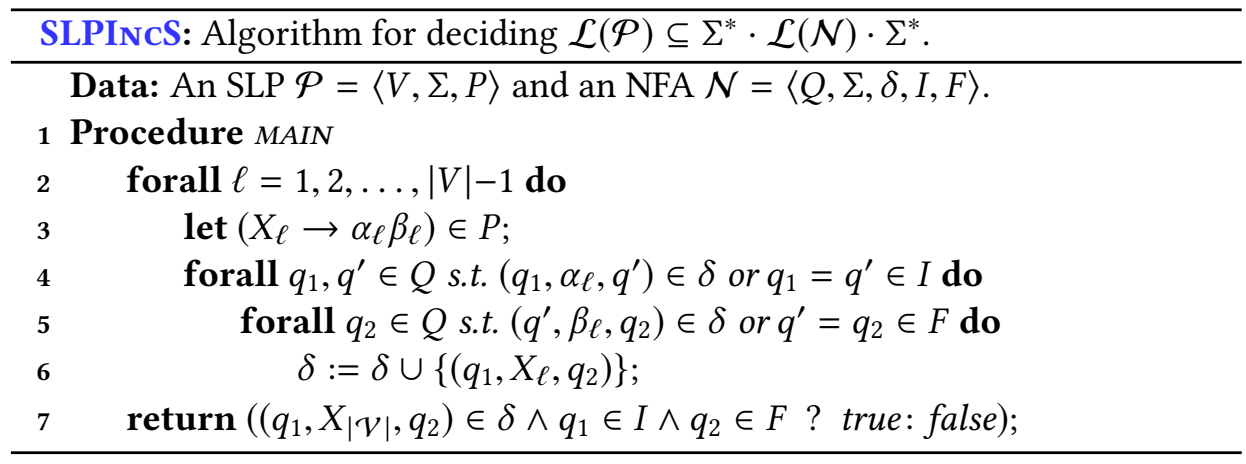

\subsection{Counting Algorithm}

State of the art tools for regular expression search are equipped with a number of features ${ }^{4}$ to perform different operations beyond deciding the existence of a match in the text. Among the most relevant of these features we find counting. Tools like grep ${ }^{5}, \mathrm{rg}^{6}, \mathrm{ack}^{7} \mathrm{or} \mathrm{ag}^{8}$ report the number of lines containing a match, ignoring matches across lines. Next we extend Algorithm SLPINCS to perform this sort of counting.

Let $\mho$ denote the new-line delimiter and let $\widehat{\Sigma}=\Sigma \backslash\{\hookleftarrow\}$. Given a string $w \in \Sigma^{+}$compressed as an $\operatorname{SLP} \mathcal{P}=\langle V, \Sigma, P\rangle$ and an automaton $\mathcal{N}=\langle Q, \widehat{\Sigma}, \delta, I, F\rangle$ built from a regular expression, Algorithm CountLines reports the number of lines in $w$ containing a match for the expression. Note that, as the tools mentioned in the previous paragraph, we deliberately ignore matches across lines.

As an overview, our algorithm computes some counting information for each alphabet symbol of the grammar (procedure INIT_AUTOMATON) which is then propagated, in a bottom-up manner, to the axiom rule. Such propagation is

\footnotetext{
${ }^{4}$ https://beyondgrep.com/feature-comparison/

${ }^{5}$ https://www.gnu.org/software/grep

${ }^{6} \mathrm{https} / / /$ github.com/BurntSushi/ripgrep

${ }^{7}$ https://github.com/beyondgrep/ack2

${ }^{8}$ https://geoff.greer.fm/ag/
} 
achieved by iterating through the grammar rules (loop in line 14) and combining, for each rule, the information for the symbols on the right hand side to obtain the information for the variable on the left (procedure counT). Finally, the output of the algorithm is computed from the information propagated to the axiom symbol (line 22).

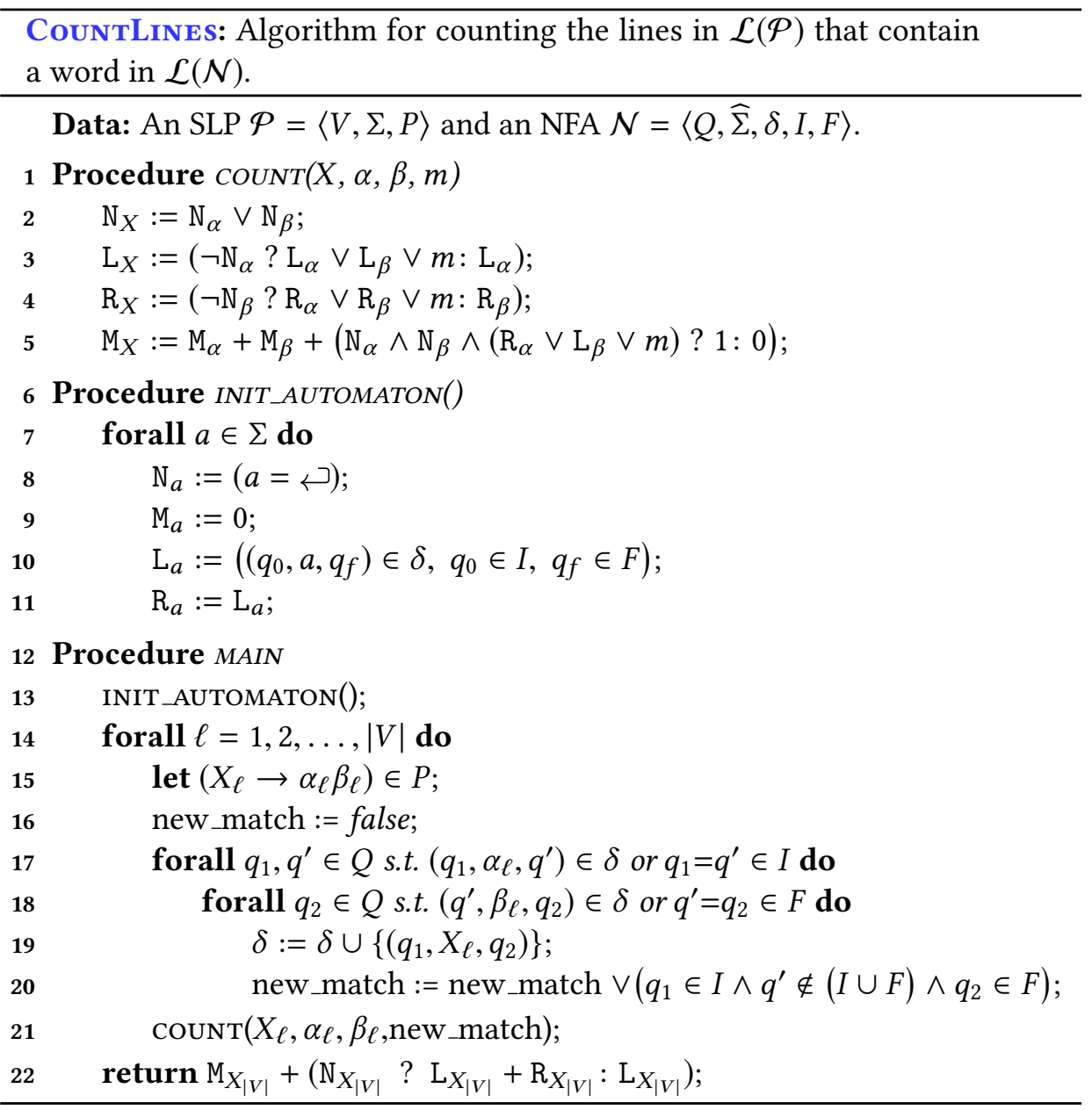

Define a line as a maximal factor of $w$ each symbol of which belongs to $\widehat{\Sigma}$, a closed line as a line which is not a prefix nor a suffix of $w$ and a matching line as a line in $\overline{\mathcal{L}(\mathcal{N})}$, where $\overline{\mathcal{L}(\mathcal{N})}=\widehat{\Sigma}^{*} \cdot \mathcal{L}(\mathcal{N}) \cdot \widehat{\Sigma}^{*}$.

Example 5.2.1. Consider the word $\left.w=" a b_{\hookleftarrow}\right\lrcorner a_{\hookleftarrow} b_{a} b_{\hookleftarrow}$ ” and an NFA $\mathcal{N}$ with $\mathcal{L}(\mathcal{N})=\{b a\}$. Then the strings " $a b$ ", " $a$ " and "bab" are lines of which only the strings " $a b$ " and " $a$ " are closed lines and " $b a b$ " is the only matching line. 
Definition 5.2.2 (Counting Information). Let $\mathcal{N}$ be an NFA and let $\mathcal{P}=\langle\mathcal{V}, \Sigma, P\rangle$ be an SLP. The counting information of $\tau \in(V \cup \Sigma)$, with $\tau \Rightarrow^{*} u$ and $u \in \Sigma^{+}$, is the tuple $C_{\tau} \stackrel{\text { def }}{=}\left\langle\mathrm{N}_{\tau}, \mathrm{L}_{\tau}, \mathrm{R}_{\tau}, \mathrm{M}_{\tau}\right\rangle$ where

$$
\begin{array}{ll}
\mathrm{N}_{\tau} \stackrel{\text { def }}{=} \exists k ;(u)_{k}=\prec & \mathrm{L}_{\tau} \stackrel{\text { def }}{=} \exists i,(u)_{1, i} \in \widehat{\Sigma}^{*} \cdot \mathcal{L}(\mathcal{N}) \\
\mathrm{R}_{\tau} \stackrel{\text { def }}{=} \exists j,(u)_{j, \dagger} \in \mathcal{L}(\mathcal{N}) \cdot \widehat{\Sigma}^{*} & \mathrm{M}_{\tau} \stackrel{\text { def }}{=}\left|\left\{(i+1, j-1) \mid(u)_{i, j} \in \hookleftarrow \cdot \overline{\mathcal{L}(\mathcal{N})} \cdot \hookleftarrow\right\}\right|
\end{array}
$$

Note that $\mathrm{N}_{\tau}, \mathrm{L}_{\tau}$ and $\mathrm{R}_{\tau}$ are boolean values while $\mathrm{M}_{\tau}$ is an integer. It follows from the definition that the number of matching lines in $u$, with $\tau \Rightarrow^{*} u$, is given by the number of closed matching lines $\left(\mathrm{M}_{\tau}\right)$ plus the prefix of $u$ iff it is a matching line $\left(\mathrm{L}_{\tau}\right)$ and the suffix of $u$ iff it is a matching line $\left(\mathrm{R}_{\tau}\right)$ different from the prefix $\left(\mathrm{N}_{\tau}\right)$. Since whenever $\mathrm{N}_{\tau}=$ false we have $\mathrm{L}_{\tau}=\mathrm{R}_{\tau}$, it follows that

$$
\sharp \text { matching lines in } u=M_{\tau}+\left\{\begin{array}{ll}
1 & \text { if } \mathrm{L}_{\tau} \\
0 & \text { otherwise }
\end{array}+ \begin{cases}1 & \text { if } \mathrm{N}_{\tau} \wedge \mathrm{R}_{\tau} \\
0 & \text { otherwise }\end{cases}\right.
$$

Computing the counting information of $\tau$ requires deciding membership of certain factors of $u$ in $\overline{\mathcal{L}(A)}$. As explained before, we reduce these membership queries to language inclusion checks which are solved by Algorithm SLPINCS. This operation corresponds to lines 17 to 19 of Algorithm CountLines. As a result, after processing the rule for $\tau$, we have $\left(q_{1}, \tau, q_{2}\right) \in \delta$ iff the automaton moves from $q$ to $q^{\prime}$ reading (a) $u$, (b) a suffix of $u$ and $q_{1} \in I$, or (c) a prefix of $u$ and $q_{2} \in F$.

Procedures COUNT and INIT_AUTOMATON are quite straightforward, the main difficulty being the computation of $\mathrm{M}_{X}$ which we explain next. Let $x, y \in \Sigma^{+}$be the strings generated by $\alpha$ and $\beta$, respectively. Given rule $X \rightarrow \alpha \beta, X$ generates all the matching lines generated by $\alpha$ and $\beta$ plus, possibly, a "new" matching line of the form $z=(x)_{i, \dagger}(y)_{1, j}$ with $1<i \leq|x|$ and $1 \leq j<|y|$. Such an extra matching line appears iff both $\alpha$ and $\beta$ generate a $\checkmark$ symbol and one of the following holds:

(a) The suffix of $x$ matches the expression.

(b) The prefix of $y$ matches the expression.

(c) There is a new match $m \in z$ with $m \notin x, m \notin y$ (line 20).

Example 5.2.3. Let $\mathcal{N}$ be an automaton with $\mathcal{L}(\mathcal{N})=\{a b, b a\}$ and let $X \rightarrow \alpha \beta$ be a grammar rule with $\alpha \Rightarrow^{*} b a \hookleftarrow a$ and $\beta \Rightarrow^{*} b \hookleftarrow a b a$. Then $X \Rightarrow^{*} b a \hookleftarrow a b \hookleftarrow a b a$.

The matching lines generated by $\alpha, \beta$ and $X$ are, respectively, $\{b a\},\{a b a\}$ and $\{b a, a b, a b a\}$. Furthermore

$$
C_{\alpha}=\langle\text { true, true, false, } 0\rangle \quad \text { and } \quad C_{\beta}=\langle\text { true, false, true, } 0\rangle .
$$


Therefore, applying function counT we find that $C_{X}=\langle$ true, true, true, 1$\rangle$. Therefore the number of matching lines is $1+1+1=3$, as expected.

Note that the counting information computed by Algorithm CountLines can be used to uncompress only the matching lines by performing a top-down processing of the SLP. For instance, given $X \rightarrow \alpha \beta$ with $C_{X}=\langle$ true, true, false, 0$\rangle$ and $C_{\alpha}=\langle$ true, true, false, 0$\rangle$, there is no need to decompress the string generated by $\beta$ since we are certain it is not part of any matching line (otherwise we should have $\mathrm{M}_{X}>0$ or $\mathrm{R}_{X}=$ true).

Next, we describe the data structures that we use to implement Algorithm COUNTLines with nearly optimal complexity.

\subsubsection{Data Structures}

We assume the alphabet symbols, variables and states are indexed and use the following data structures, illustrated in Figure 5.3: an array $\mathcal{A}$ with $t+|\Sigma|$ elements, where $t$ is the number of rules of the SLP, and two $s \times s$ matrices $\mathcal{M}$ and $\mathcal{K}$ where $s$ is the number of states of the automaton.

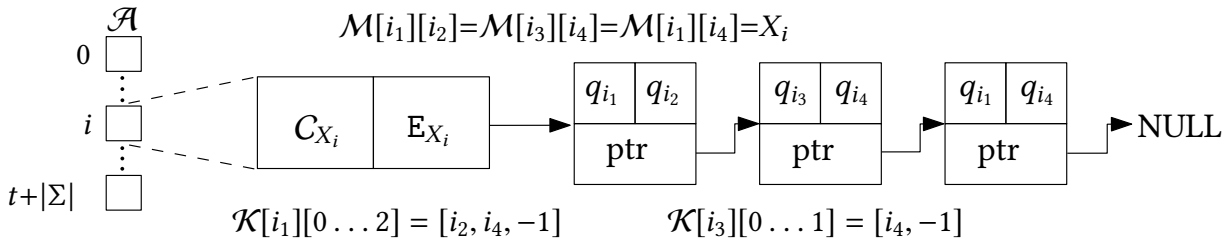

Figure 5.3: Data structures enabling nearly optimal running time for Algorithm COUntLines. The image shows the contents of $\mathcal{M}$ after processing rule $X_{i} \rightarrow \alpha_{i} \beta_{i}$ and the contents of $\mathcal{K}$ after processing $X_{\ell} \rightarrow \alpha_{\ell} \beta_{\ell}$ with $\beta_{\ell}=X_{i}$.

Each element $\mathcal{A}[i]$ contains the information related to variable $X_{i}$, i.e. $C_{X_{i}}$ and the list of transitions labeled with $X_{i}$, denoted $\mathrm{E}_{X_{i}}$. We store $C_{X}$ using one bit for each $\mathrm{N}_{X}, \mathrm{~L}_{X}$ and $\mathrm{R}_{X}$ and an integer for $\mathrm{M}_{X}$.

For each rule $X_{\ell} \rightarrow \alpha_{\ell} \beta_{\ell}$ the matrix $\mathcal{K}$ is set so that row $i$ contains the set of states reachable from the state $q_{i}$ by reading the string generated by $\beta_{\ell}$, i.e. $\mathcal{K}[i]=\left\{q_{j} \mid\left(q_{i}, \beta_{\ell}, q_{j}\right) \in \delta\right\}$. If there are less than $s$ such states we use a sentinel value ( -1 in Figure 5.3).

Finally, each element $\mathcal{M}[i][j]$ stores the index $\ell$ of the last variable for which $\left(q_{i}, X_{\ell}, q_{j}\right)$ was added to $\delta$. Note that since rules are processed one at a time, matrices $\mathcal{K}$ and $\mathcal{M}$ can be reused for all rules.

Observe that it is straightforward to update the matrices $\mathcal{M}$ and $\mathcal{K}$ in $O\left(s^{2}\right)$ time for each rule $X_{\ell} \rightarrow \alpha_{\ell} \beta_{\ell}$ since there are up to $s^{2}$ transitions $\left(q_{i}, \beta_{\ell}, q_{j}\right) \in \delta$. These data structures provide $O(1)$ runtime for the following operations: 
- Accessing the information corresponding to $\alpha_{\ell}$ and $\beta_{\ell}$ at line 15 (using $\mathcal{A}$ ).

- Accessing the list of pairs $\left(q, q^{\prime}\right)$ with $\left(q, \alpha_{\ell}, q^{\prime}\right) \in \delta$ at line 17 (using $\left.\mathrm{E}_{X_{i}}\right)$.

- Accessing the list of states $q_{2}$ with $\left(q^{\prime}, \beta_{\ell}, q_{2}\right) \in \delta$ at line 18 (using $\mathcal{K}$ ).

- Inserting a pair $\left(q, q^{\prime}\right)$ in $\mathrm{E}_{X_{i}}$ (avoiding duplicates) at line 19 (using $\mathcal{M}$ ).

As a result, Algorithm CountLines runs in $O\left(t \cdot s^{3}\right)^{9}$ time using $O\left(t \cdot s^{2}\right)$ space when the automaton built from the regular expression is an NFA and it runs in $O(t \cdot s)$ time and $O(t \cdot s)$ space when the automaton is a DFA (each row of $\mathcal{K}$ stores up to one state, hence the loop in line 18 results in, at most, one iteration).

Abboud et al. [2017, Thm. 3.2] proved that, under the Strong Exponential Time Hypothesis, there is no combinatorial algorithm for deciding whether a grammar-compressed text contains a match for a DFA running in $O\left((t \cdot s)^{1-\varepsilon}\right)$ time with $\varepsilon>0$. For NFAs, they proved [Abboud et al. 2017, Thm. 4.2] that, under the $k$-Clique Conjecture, there is no combinatorial algorithm running in $O\left(\left(t \cdot s^{3}\right)^{1-\varepsilon}\right)$ time. Therefore, our algorithm is nearly optimal in both scenarios.

\subsection{Implementation}

We implemented Algorithm CountLines, using the data structures described in the previous section, in a tool named zearch ${ }^{10}$. Our tool works on repair ${ }^{11}$ compressed text and, beyond counting the matching lines, it can also report them by partially decompressing the input file. The implementation consists of less than 2000 lines of $\mathrm{C}$ code.

The choice of this particular compressor, which implements the RePair algorithm of Larsson and Moffat [1999], is due to the little effort required to adapt Algorithm CountLines to the specifics of the grammar built by repair and the compression it achieves (see Table 5.1). However zearch can handle any grammar-based compression scheme by providing a way to recover the SLP from the input file.

Recall that we assume the alphabet symbols, variables and states are indexed. For repair-compressed text, the indexes of the alphabet symbols are $0 \ldots 255(\Sigma$ is fixed $\left.{ }^{12}\right)$ and the indexes of the variables are $256 \ldots t+256$. Typically, grammarbased compressors such as repair encode the grammar so that rule $X \rightarrow \alpha \beta$ appears always after the rules with $\alpha$ and $\beta$ on the left hand side. Thus, each iteration of the loop in line 15 reads a subsequent rule from the compressed input.

\footnotetext{
${ }^{9}$ The algorithm performs $t$ iterations of loop in line 15, up to $s^{2}$ iterations of loop in line 17 and up to $s$ iterations for loop in line 18 .

${ }^{10} \mathrm{https}$ //github.com/pevalme/zearch

${ }^{11}$ https://storage.googleapis.com/google-code-archive-downloads/v2/code.google.com/ re-pair/repair110811.tar.gz

${ }^{12}$ Our algorithm also applies to larger alphabets, such as UTF8, without altering its complexity.
} 
We translate the input regular expression into an $\varepsilon$-free NFA using the automata library libfa ${ }^{13}$ which applies Thompson's algorithm [Thompson 1968] with on-the-fly $\varepsilon$-removal.

\subsection{Empirical Evaluation}

Next we present a summary of the experiments carried out to assess the performance of zearch. The details of the experiments, including the runtime and number of matching lines reported for each expression on each file and considering more tools, file sizes and regular expressions are available on-line ${ }^{14}$, where we report graphs as the ones shown in Figure 5.4. The following explanations about how the experiments reported in this thesis were carried out also apply to the larger set of experiments available on-line.

All tools for regular expression searching considered in this benchmark are used to count the matching lines without reporting them. As expected, all tools report the exact same result for all benchmarks. To simplify the terminology, we refer to counting the matching lines as searching, unless otherwise stated.

\subsubsection{Tools}

Our benchmark compares the performance of zearch against the fastest implementations we found for the following operations:

(i) Searching the compressed text without decompression.

(ii) Searching the uncompressed text.

(iii) Decompressing the text without searching.

(iv) Searching the uncompressed text as it is recovered by the decompressor.

For searching the compressed text we consider GNgrep, the tool developed by Navarro [2003] for searching on text compressed with the grammar-based compressor LZW defined by Welch [1984]. To the best of our knowledge, this is the only existing tool departing from the decompress and search approach.

For searching uncompressed text we consider grep and hyperscan. We improve the performance of grep by compiling it without perl regular expression compatibility, which is not supported by zearch. We used the library hyperscan by means of the tool (provided with the library) simplegrep, which we modified $^{15}$ to efficiently read data either from stdin or an input file. These tools are

\footnotetext{
${ }^{13} \mathrm{http}: / /$ augeas.net/libfa/index.html

${ }^{14}$ https://pevalme.github.io/zearch/graphs/index.html

${ }^{15}$ https://gist.github.com/pevalme/f94bedc9ff08373a0301b8c795063093
} 


\section{Regular Expressions}

r1: ".", r2: "wosel", r3: "port", r4: "20[0-9]\{2\}", r5: "([0-9]\{3\}1.)\{3\}[0-9]", r6: "[0-9]\{4\}", r7: "([a-z]+1.)+[a-z]+ - -", r8: "GET *" ([13-9]|2[1-9]|2-[1-9])",

r9: "(([0-9])|([0-2][0-9])|([3][0-1]))/(Jan|Feb|Mar|Apr|May|Jun|Jul|Aug|Sep|Oct|Nov|Dec)/[0-9]\{4\}", r10: "(([0-9])|([0-2][0-9])|([3][0-1]))-(Jan|Feb|Mar|Apr|May|Jun|Jul|Aug|Sep|Oct|Nov|Dec)-[0-9]\{4\}",

\section{Graphs}

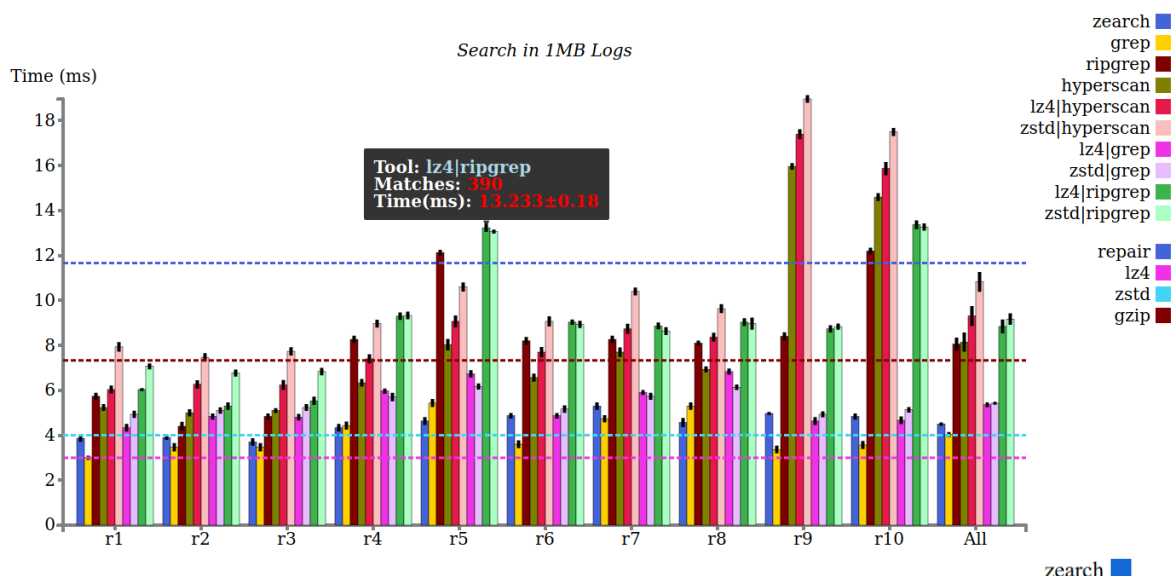

Cactus diagram for searching in $1 M B \operatorname{Logs}$

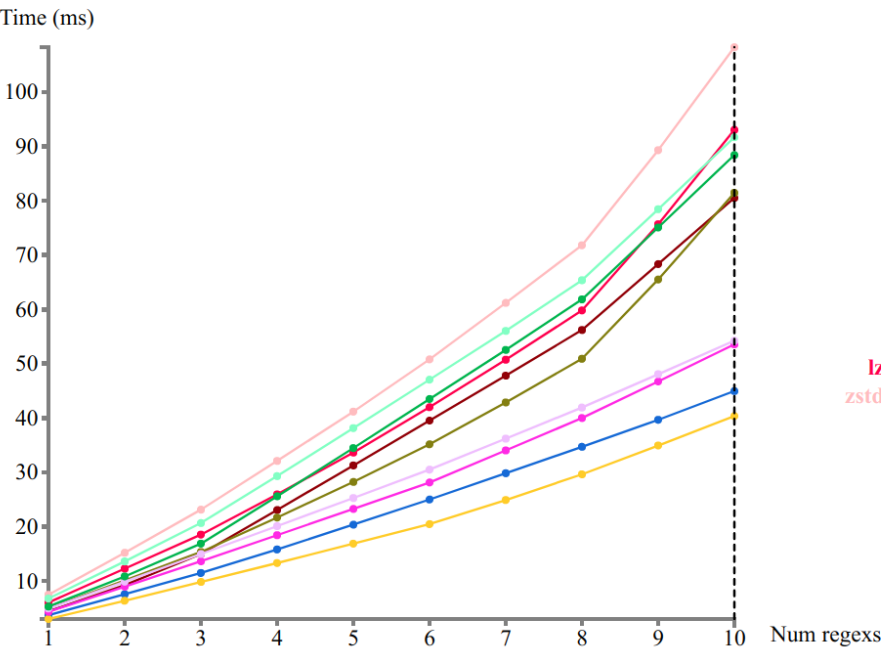

ripgrep

hyperscan

lz4|hyperscan zstd|hyperscan

lz4|grep zstd|grep lz4|ripgrep zstd|ripgrep

zearch: $\mathbf{4 4 . 9 6 7}$

grep: 40.36 '

ripgrep: $\mathbf{8 0 . 5 3 3}$

hyperscan: 81.466

lz4|hyperscan: 93.067

Iz4|grep: $\mathbf{5 3 . 5 6 6}$

zstd|grep: 54.23

lz4|ripgrep: 88.432

zstd|ripgrep: 91.7

Figure 5.4: The first graph shows the time required to report the number of lines in a log file matching a regular expression. All tools are fed with the same regular expression. The decompress and search approach is implemented in parallel i.e. searching on the output uncompressed text as it is recovered by the decompressor. As a reference, we show the time required for decompressing the file with different tools (horizontal lines). The second graph is the cactus plot corresponding the the data from the first graph. In this case, we observe that zearch is faster than any other tool, except grep. 
top of the class ${ }^{16}$ for regular expression searching.

For (de)compressing the files we use zstd and $1 z 4$ which are among the best lossless compressors ${ }^{17}$, being $1 z 4$ considerably faster while $z$ std achieves better compression. We use both tools with the highest compression level, which has little impact on the time required for decompression.

We use versions grep v3.3, hyperscan v5.0.0, lz4 v1.8.3 and zstd v1.3.6 running in an Intel Xeon E5640 CPU $2.67 \mathrm{GHz}$ with $20 \mathrm{~GB}$ RAM which supports SIMD instructions up to SSE4-2. We restrict to ASCII inputs and set LC_ALL=C for all experiments, which significantly improves the performance of grep. Since both hyperscan and GNgrep count positions of the text where a match ends, we extend each regular expression (when used with these tools) to match the whole line. We made this decision to ensure all tools solve the same counting problem and therefore produce the same output.

\subsubsection{Files and Regular Expressions}

Our benchmark consists of an automatically generated $\log ^{18}$ of HTTP requests, English Subtitles [Lison and Tiedemann 2016], and a concatenation of English $B o o k s^{19}$. Table 5.1 shows how each compressor behaves on these files.

\begin{tabular}{|c|c|c|c|c|c|c|c|c|c|c|c|c|c|}
\hline & \multirow[b]{2}{*}{ File } & \multicolumn{4}{|c|}{ Compressed size } & \multicolumn{4}{|c|}{ Compression time } & \multicolumn{4}{|c|}{ Decompression time } \\
\hline & & LZW & repair & zstd & $1 z 4$ & LZW & repair & zstd & $1 z 4$ & LZW & repair & zstd & $1 z 4$ \\
\hline \multirow{6}{*}{ 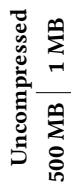 } & Logs & 0.19 & 0.08 & 0.07 & 0.12 & 0.04 & 0.19 & 0.51 & 0.03 & 0.02 & 0.01 & 0.01 & 0.004 \\
\hline & Subtitles & 0.36 & 0.13 & 0.11 & 0.15 & 0.04 & 0.25 & 0.3 & 0.03 & 0.02 & 0.01 & 0.01 & 0.004 \\
\hline & Books & 0.42 & 0.34 & 0.27 & 0.43 & 0.04 & 0.29 & 0.42 & 0.08 & 0.02 & 0.02 & 0.01 & 0.004 \\
\hline & $\log s$ & 96 & 38 & 33 & 65 & 16.9 & 123.2 & 819.1 & 13.3 & 7.8 & 5.5 & 1.1 & 0.64 \\
\hline & Subtitles & 191 & 66 & 55 & 114 & 19.9 & 169.3 & 415.2 & 22.8 & 8.6 & 8.2 & 1.2 & 0.81 \\
\hline & Books & 206 & 153 & 129 & 216 & 20.2 & 198.6 & 646.3 & 40.6 & 8.6 & 9.7 & 2.0 & 0.8 \\
\hline
\end{tabular}

Table 5.1: Sizes (in MB) of the compressed files and (de)compression times (in seconds). Maximum compression levels enabled. $($ Blue $=$ best; bold black = second best; red $=$ worst $)$.

We first run each experiment 3 times as warm up so that the files are loaded in memory. Then we measure the running time 30 times and compute the confidence interval (with $95 \%$ confidence) for the running time required to count the number of matching lines for a regular expression in a certain file using a certain tool.

We consider the point estimate of the confidence interval and omit the margin of error which never exceeds the $9 \%$ of the point estimate for the reported experiments. The on-line version of these experiment does report the margin

\footnotetext{
${ }^{16}$ https://rust-leipzig.github.io/regex/2017/03/28/comparison-of-regex-engines/

${ }^{17}$ https://quixdb.github.io/squash-benchmark/

${ }^{18} \mathrm{http} / / /$ ita.ee.lbl.gov/html/contrib/NASA-HTTP.html

${ }^{19}$ https://web.eecs.umich.edu/ lahiri/gutenberg_dataset.html
} 
of error as a black mark on the top of each bar. The height of the bar is the point estimate computed for the given experiment while the black mark denotes the confidence interval (see Figure 5.4). Figure 5.5 summarizes the obtained results when considering, for all files, the regular expressions: "what", "HTTP”, ".", "I .* you ", " $[a-z]\{4\}$ ", " $[a-z] *[a-z]\{3\}$ ", "[0-9]\{4\}”, "[0-9] $\{2\} /$ (Jun | Jul | Aug)/[0-9] \{4\}".

For clarity, we report only on the most relevant tools among the ones considered. For $1 z 4$ and zstd, we report the time required to decompress the file and send the output to /dev/null.
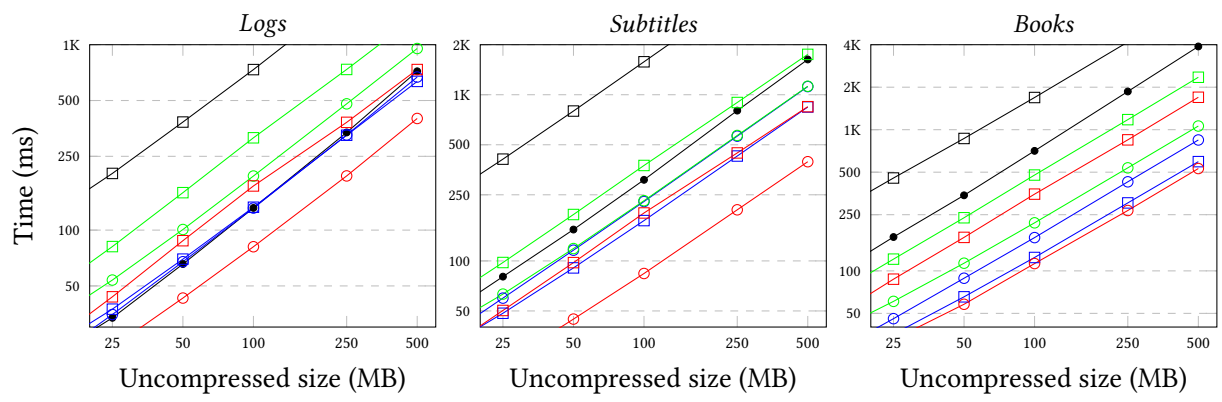

$\multimap$ zearch $\multimap$ grep $\square$ hyperscan

zstd|hyperscan $\odot$ lz4|hyperscan $\odot$ lz4 $\square$ zstd $\square$ GNgrep

Figure 5.5: Average running time required to report the number of lines matching a regular expression in a file and time required for decompression. Colors indicate whether the tool performs the search on the uncompressed text (blue); the compressed text (black); the output of the decompressor (green); or decompresses the file without searching (red).

\subsubsection{Analysis of the Results.}

Figure 5.5 and Table 5.1 show that the performance of zearch improves with the compression ratio. This is to be expected since zearch processes each grammar rule exactly once and better compression results in less rules to be processed. In consequence, zearch is the fastest tool for counting matching lines in compressed $\log$ files while it is the second slowest one for the Books.

In particular, zearch is more than $25 \%$ faster than any other tool working on compressed Log files. Actually zearch is competitive with grep and hyperscan, even though these tools operate on the uncompressed text. These results are remarkable since hyperscan, unlike zearch, uses algorithms specifically designed to take advantage of SIMD parallelization. ${ }^{20}$

Finally, the fastest tool for counting matching lines in compressed Subtitles and Books, $1 \mathrm{z} 4$ / hyperscan, applies to files larger than the ones obtained when

\footnotetext{
${ }^{20}$ According to the documentation, hyperscan requires, at least, support for SSSE3.
} 
compressing the data with repair (see Table 5.1). However, when considering a better compressor such as zstd, which achieves slightly more compression than repair, the decompression becomes slower. As a result, zearch outperforms zstd | hyperscan by more than 7\% for Subtitles files and 50\% for Logs.

\section{Contrived Example}

Next, we discharge the full potential of our approach by considering a contrived experiment in which the data is highly repetitive. In particular, we consider a file where all lines are identical and consist of the sentence "This is a contrived experiment. $\longleftrightarrow$ ”. Table 5.2 shows the compression achieved on this data for each of the compressors.

\begin{tabular}{|c|c|c|c|c|c|c|c|c|c|c|c|c|}
\hline \multirow[b]{2}{*}{ File size } & \multicolumn{4}{|c|}{ Compressed size } & \multicolumn{4}{|c|}{ Compression time } & \multicolumn{4}{|c|}{ Decompression time } \\
\hline & LZW & repair & zstd & $1 z 4$ & LZW & repair & zstd & $1 z 4$ & LZW & repair & zstd & $1 z 4$ \\
\hline $1 \mathrm{MB}$ & 13 & 0.072 & 0.135 & 4.1 & 0.0 & 0.08 & 0.01 & 0.004 & 0.01 & 0.01 & 0.003 & 0.003 \\
\hline $500 \mathrm{MB}$ & 950 & 0.09 & 44 & 2000 & 14.5 & 53.1 & 0.9 & 0.28 & 3.9 & 3.3 & 0.24 & 0.2 \\
\hline
\end{tabular}

Table 5.2: Sizes (in KB) of the compressed files and (de)compression times (in seconds). Maximum compression levels enabled. $($ Blue $=$ best; bold black $=$ second best; red $=$ worst $)$.

As expected this contrived file results in really high compression ratios. As we show next, this scenario evidences the virtues of zearch which is capable of searching in $500 \mathrm{MB}$ of data by processing a grammar consisting of 57 rules.

Table 5.3 summarizes the results obtained when searching the $500 \mathrm{MB}$ contrived file for different regular expressions. ${ }^{21}$ For each expression, we report the time required to (i) search on the compressed data without decompression, (ii) search on the uncompressed data and (iii) search with the best implementation of the parallel decompress and search approach. ${ }^{22}$

As shown by Tables 5.2 and 5.3, our tool is about 10 times faster at searching than $1 z 4$ at decompression. Therefore, zearch clearly outperforms any decompress and search approach, even if decompression and search are done in parallel. This is to be expected since zearch only needs to process 90 Bytes of data (the size of the grammar) while the rest of the tools need to process $500 \mathrm{MB}$.

Similarly, GNgrep processes $950 \mathrm{~KB}$ of data (the size of the LZW-compressed data). As a consequence, when there are no matches of the expression, GNgrep is faster than decompression as evidenced by the last two rows of Table 5.3. However, GNgrep reports the number of matching lines by explicitly finding the pos-

\footnotetext{
${ }^{21}$ We run each experiment 30 times and report the point estimate of the confidence interval with $95 \%$ confidence.

${ }^{22}$ The best implementation might vary depending on the expression.
} 


\begin{tabular}{|c|c|c|c|c|}
\hline Expression & zearch & GNgrep / grep & | hyperscan | decompress & and search \\
\hline "experiment" & | 2.267 & $14 \mathrm{~K} \mid 1352$ & $1784 \mid$ & 1652 \\
\hline "This" & | 2.533 & $14 \mathrm{~K}$ | 764 & 2166 | & 959 \\
\hline “.” & | 2.467 & $14 \mathrm{~K}$ | 703 & 1276 & 886 \\
\hline "[a-z] $\{4\} "$ & | 2.667 & 14K | 1138 & 1270 | & 1360 \\
\hline$"[a-z]\{11\} "$ & | 2.233 & $37 \mid 1690$ & 1312 | & 1397 \\
\hline "That" & | 2.433 & 37.2 | 607 & 239 | & 444 \\
\hline
\end{tabular}

Table 5.3: Time (ms) required to report the number of lines matching a regular expression in the $500 \mathrm{MB}$ large contrived file. $($ Blue = fastest; bold black = second fastest; red = slowest).

itions in the data where the match begins, which results rather inefficient when all lines of the file contain a match, as evidenced by the first 4 rows of Table 5.3.

\subsection{Fine-Grained Analysis of the Implementation}

The grammars produced by repair break the definition of SLP in behalf of compression by allowing the axiom rule to have more than two symbols on the right hand side. This is due to the fact that the axiom rule is built with the remains of the input text after creating all grammar rules.

Typically, the length of the axiom is larger or equal than the number of rules in the SLP so the way in which the axiom is processed heavily influences the performance of zearch.

On the other hand, our experiments show that the performance of zearch is typically far from its worst case complexity. This is because the worst case scenario assumes each string generated by a grammar variable labels a path between each pair of states of the automaton. However, we only observed such behavior in contrived examples.

\subsubsection{Processing the Axiom Rule.}

Algorithm CountLines could process the axiom rule $X_{|V|} \rightarrow \sigma$ by building an SLP with the set of rules

$$
\left\{S_{1} \rightarrow(\sigma)_{1}(\sigma)_{2}\right\} \cup\left\{S_{i} \rightarrow S_{i-1}(\sigma)_{i+1}|i=2 \ldots| \sigma \mid-2\right\} \cup\left\{X_{|V|} \rightarrow S_{|\sigma|-2}(\sigma)_{\dagger}\right\}
$$

However it is more efficient to compute the set of states reachable from the initial ones when reading the string generated with $S_{1}$ and update this set for each symbol $(\sigma)_{i}$. To perform the counting note that $C_{S_{i}}$ is only used to compute $C_{S_{i+1}}$ and can be discarded afterwards. This yields an algorithm running in 
$\mathcal{O}\left(|V| \cdot s^{3}+|\sigma| \cdot s^{2}\right)$ time using $\mathcal{O}\left(|V| \cdot s^{2}\right)$ space where $|V|$ is the number of rules of the input grammar and $X_{|V|} \rightarrow \sigma$ its axiom.

\subsubsection{Number of Operations Performed by the Algorithm}

Define $s_{\tau, q}=\left|\left\{q^{\prime} \mid\left(q, \tau, q^{\prime}\right) \in \delta\right\}\right|$ and $s_{\tau}=\sum_{q \in Q} s_{\tau, q}$ and let us recall the complexity of Algorithm CountLines according to the data structures described in Section 5.2.1. The algorithm iterates over the $|V|$ rules of the grammar and, for each of them:

(i) Initializes matrix $\mathcal{K}$ with $s_{\beta_{\ell}}$ elements $^{23}$

(ii) Iterates through $\mathcal{K}\left[q^{\prime}\right]\left[0 \ldots s_{\beta_{\ell}, q^{\prime}}\right]$ for each pair $\left(q_{1}, q^{\prime}\right) \in \mathrm{E}_{\alpha_{\ell}}$.

Then it processes the axiom rule iterating, for each symbol $(\sigma)_{i}$, through $s_{(\sigma)_{i}}$ transitions.

These are all the operations performed by the algorithm with running time dependent on the size of the input. Hence, Algorithm CountLines runs in

$$
O\left(\sum_{\ell=1}^{|V|} \tilde{s}_{\ell}+\sum_{i=1}^{|\sigma|} s_{(\sigma)_{i}}\right) \text { time, where } \tilde{s}_{\ell}=s_{\beta_{\ell}}+s+\sum_{\left(q_{1}, q^{\prime}\right) \in \mathrm{E}_{\alpha_{\ell}}}\left(1+s_{\beta_{\ell}, q^{\prime}}\right) p .
$$

Note that $\tilde{s}_{\ell} \leq s^{3}, s_{(\sigma)_{i}} \leq s^{2}$ so the worst case time complexity of the algorithm is $O\left(|V| \cdot s^{3}+|\sigma| \cdot s^{2}\right)$. However, in the experiments we observed that $\tilde{s}_{\ell}$ and $s_{\left(\sigma_{i}\right)}$ are usually much smaller than $s^{3}$ and $s^{2}$, respectively, as reported in Table 5.4.

\begin{tabular}{|c|c|c|c|c|c|c|c|c|c|c|c|c|c|}
\hline \multirow{2}{*}{ Expression } & \multirow[b]{2}{*}{$S$} & \multirow{2}{*}{$s^{3}$} & \multicolumn{5}{|c|}{ percentiles for $\tilde{s}_{\ell}$} & \multirow[t]{2}{*}{$s^{2}$} & \multicolumn{5}{|c|}{ percentiles for $s_{(\sigma)_{i}}$} \\
\hline & & & $50 \%$ & $75 \%$ & $95 \%$ & $98 \%$ & $100 \%$ & & $50 \%$ & $75 \%$ & $95 \%$ & $98 \%$ & $100 \%$ \\
\hline "what" & 5 & 125 & 0 & 0 & 1 & 1 & 9 & 25 & 0 & 0 & 1 & 1 & 2 \\
\hline “HTTP" & 5 & 125 & 0 & 0 & 0 & 0 & 10 & 25 & 0 & 0 & 0 & 0 & 2 \\
\hline “.” & 2 & 8 & 0 & 0 & 0 & 0 & 4 & 4 & 0 & 0 & 0 & 0 & 1 \\
\hline "I * you" & 9 & 729 & 3 & 13 & 16 & 18 & 29 & 81 & 3 & 3 & 5 & 5 & 9 \\
\hline$"[a-z] 4 "$ & 7 & 343 & 2 & 10 & 11 & 12 & 18 & 49 & 1 & 1 & 2 & 2 & 4 \\
\hline$"[a-z] *[a-z] 3 "$ & 7 & 343 & 3 & 11 & 14 & 18 & 31 & 49 & 1 & 3 & 3 & 4 & 8 \\
\hline$"[0-9]\{4\} "$ & 6 & 216 & 8 & 8 & 8 & 8 & 18 & 36 & 1 & 1 & 1 & 1 & 5 \\
\hline “. * A $-\mathrm{Za}-\mathrm{Z}]\{5\} ”$ & 7 & 343 & 14 & 25 & 48 & 48 & 48 & 49 & 11 & 14 & 14 & 14 & 14 \\
\hline “. * $\mathrm{A}-\mathrm{Za}-\mathrm{z}]\{10\} "$ & 12 & 1728 & 29 & 51 & 86 & 95 & 98 & 144 & 16 & 26 & 29 & 29 & 29 \\
\hline “. *[A-Za-z ] $\{20\} "$ & 22 & 10648 & 57 & 87 & 132 & 153 & 198 & 484 & 23 & 38 & 52 & 58 & 59 \\
\hline "((((.)*.)*.)*. $) * "$ & 6 & 216 & 12 & 29 & 209 & 209 & 209 & 36 & 29 & 29 & 29 & 29 & 29 \\
\hline$"((((() *) *) *) *) * "$. & 7 & 343 & 14 & 34 & 249 & 249 & 249 & 49 & 34 & 34 & 34 & 34 & 34 \\
\hline
\end{tabular}

Table 5.4: Analysis of the values $\tilde{s}_{\ell}$ and $s_{(\sigma)_{i}}$ obtained when considering different regular expressions to search Subtitles (100 MB uncompressed long). The fifth column of the fourth row indicates that when considering the expression "I .* you", for 75\% of the grammar rules we have $\tilde{s}_{\ell} \leq 13$ while $s^{3}=729$.

\footnotetext{
${ }^{23}$ We need to set up to $s$ sentinel values for the rows in $\mathcal{K}$ not used for storing $s_{\beta_{\ell}}$
} 
As the experiments show, zearch exhibits almost linear behavior with respect to the size of the automaton built from the expression. Nevertheless, there are regular expressions that trigger the worst case behavior (last two rows in Table 5.4), which cannot be avoided due to the result of Abboud et al. [2017] described before.

\subsection{Fine-Grained Complexity}

In Section 5.2 we obtained upper bounds for the worst-case time complexity of our algorithm depending on whether the automata built from the expression is an NFA or a DFA.

However, we observed in Section 5.5 that the actual behavior of our implementation is, in general, far from its worst-case scenario (see Table 5.4). This is due to the fact that the worst-case scenario assumes an NFA where each pair of states are connected by a transition for each symbol in the alphabet but this is rarely the type of automata obtained from non-contrived regular expressions.

This difference between the worst-case time complexity of the algorithm an its behavior in practice also appears when considering the problem of searching with regular expressions on plain text. Indeed, this problem led Backurs and Indyk [2016] to analyze the complexity of searching on plain text for different classes of regular expressions.

In their work, Backurs and Indyk [2016] restrict themselves to homogeneous regular expressions, i.e. regular expressions in which operators at the same level of the formula are equal ${ }^{24}$, which are grouped in classes depending on the sequence of the operators involved. Then, they obtain a lower bound for the search complexity for each class of expressions by building reductions from the Orthogonal Vector Problem (OVP for short) which, given two sets of vectors $A, B \subseteq\{0,1\}^{d}$ in $d$ dimensions, with $N$ and $M$ elements respectively, asks whether there exists $a \in A$ and $b \in B$ such that $a \cdot b=0$.

Conjecture (OV Conjecture [Bringmann and Künnemann 2015]). There are no reals $\varepsilon, d>0$ such that the OVP in $d<N^{o(1)}$ dimensions with $M=\Theta\left(N^{\alpha}\right)$ for $\alpha \in(0,1]$ can be solved in $O\left((N \cdot M)^{1-\varepsilon}\right)$ time.

The idea behind the conjecture is that any algorithm defying it would yield an algorithm for SAT violating the Strong Exponential Time Hypothesis.

Backurs and Indyk [2016] relied on the OV conjecture to determine whether a search problem is easy, i.e. there is an algorithm running in $O(T+s)$ time where $T$

\footnotetext{
${ }^{24}$ Write the regular expression as a tree. The expression is homogeneous if all non-leaf nodes at the same depth have the same label.
} 
is the size of the input text and $s$ is the number of states of the automaton, or hard, i.e. assuming the Strong Exponential Time Hypothesis (SETH) any algorithm has $\Omega\left((T \cdot s)^{1-\varepsilon}\right)$ time complexity with $\varepsilon>0$.

This analysis can be extended to consider searching on compressed text and decide whether our implementation is optimal on different classes of homogeneous regular expressions. To do that, we apply the following remark, inherited from Abboud et al. [2017], who used the OVP to analyze whether the decompress and solve approach can be outperformed by manipulating the compressed text for different problems.

Remark 5.6.1. Let $A=\left\{a_{1}, \ldots, a_{N}\right\} \subseteq\{0,1\}^{d}$ and $B=\left\{b_{1}, \ldots, b_{M}\right\} \subseteq\{0,1\}^{d}$ be an instance of the OVP in $d \leq N^{o(1)}$ dimensions with $M=O(N)$. We define a string $T$ with a representation as an SLP of sizet $=O(N \cdot d)$ and a regular expression $\pi$ of size $s=O(M \cdot d)$ such that the string contains a match for the expression iff we have a solution for the OVP.

If there is an algorithm for regular expression searching on compressed text that operates, for a class of regular expressions that includes $\pi$, in $O\left((t \cdot s)^{1-\varepsilon}\right)$ with $\epsilon>0$ then it would solve the OVP in $O\left((N \cdot M)^{1-\varepsilon}\right)$ (since the dimension is fixed) which contradicts the OV conjecture.

\subsubsection{Complexity of Searching on Compressed Text}

Given a regular expression, we say it is homogeneous of type "I+" iff the regular expression is a disjunction of + operators and terminals. We extend this notation to any combination of operators. For instance, the expressions " $a+b+$ " and " $a+b$ " are homogeneous of type ".+" while " $a+b *$ " is not homogeneous. Recall that the size of a regular expression is the number of operators and terminals used to define the expression. For instance, " $a+b+$ " and " $a+b$ " have size 4 and 3 , respectively.

The following three results use Remark 5.6.1 to show that the time complexity of regular expression searching on compressed text is $\Omega(t \cdot s)$, where $t$ is the size of the SLP and $s$ is the size of the expression, when the regular expression is homogeneous of type “.+" “.*” or “.।".

THEOREM 5.6.2. There is no algorithm for searching with a regular expression on grammar-compressed text that operates in $O\left((t \cdot s)^{1-\epsilon}\right)$ time with $\varepsilon>0$, where $t$ is the size of the compressed text and $s$ is the size of the regular expression, when the expression is homogeneous of type ".+". 
Proof. Let $A=\left\{a_{1}, \ldots, a_{N}\right\} \subseteq\{0,1\}^{d}$ and $B=\left\{b_{1}, \ldots, b_{M}\right\} \subseteq\{0,1\}^{d}$ be an instance of the OVP in $d \leq N^{o(1)}$ dimensions with $M=O(N)$. Without loss of generality, assume the dimension is even. Consider the regular expression

$$
\pi \stackrel{\text { def }}{=} F\left(b_{1}\right) z F\left(b_{2}\right) z \ldots z F\left(b_{M}\right) z "
$$

on the alphabet $\Sigma=\{x, y, z\}$ with $F\left(b_{i}\right) \stackrel{\text { def }}{=} f\left(b_{i}, 1\right) f\left(b_{i}, 2\right), \ldots, f\left(b_{i}, d\right)$ and

$$
f(b, j) \stackrel{\text { def }}{=}\left\{\begin{array}{lll}
x x^{+} & \text {if } \quad(b)_{j}=1 \text { and } j \text { is even } \\
x^{+} & \text {if } \quad(b)_{j}=0 \text { and } j \text { is even } \\
y y^{+} & \text {if } \quad(b)_{j}=1 \text { and } j \text { is odd } \\
y^{+} & \text {if } \quad(b)_{j}=0 \text { and } j \text { is odd }
\end{array},\right.
$$

where $(b)_{j}$ is the $j$-th component of the vector $b$. Clearly, $\pi$ is homogeneous of type ".+" and has size $s=O(M \cdot d)$.

Now, we define an SLP $\mathcal{P}$ on $\Sigma=\{x, y, z\}$ such that $\mathcal{L}(\mathcal{P})=\{w\}$ with

$$
w \stackrel{\text { def }}{=}\left((x x y y)^{\frac{d}{2}} z\right)^{M-1} \tilde{F}\left(a_{1}\right) z \ldots\left((x x y y)^{\frac{d}{2}} z\right)^{M-1} \tilde{F}\left(a_{N}\right) z\left((x x y y)^{\frac{d}{2}} z\right)^{M-1}
$$

where $\tilde{F}\left(a_{i}\right) \stackrel{\text { def }}{=} \tilde{f}\left(a_{i}, 1\right) \tilde{f}\left(a_{i}, 2\right), \ldots, \tilde{f}\left(a_{i}, d\right)$ and

$$
\tilde{f}(a, j) \stackrel{\text { def }}{=}\left\{\begin{array}{lll}
x & \text { if } & (a)_{j}=1 \text { and } j \text { is even } \\
x x & \text { if } & (a)_{j}=0 \text { and } j \text { is even } \\
y & \text { if } & (a)_{j}=1 \text { and } j \text { is odd } \\
y y & \text { if } & (a)_{j}=0 \text { and } j \text { is odd }
\end{array}\right.
$$

The substring $\left((x x y y)^{\frac{d}{2}} z\right)^{M-1}$ can be generated with an SLP of size $O(d+\log M)$, hence $w$ can be compressed as an SLP $\mathcal{P}$ of size $t=O(N \cdot d+d+\log M)$ and, since $d \leq N^{o(1)}$ is a constant and $M=O(N)$, we find that $t=O(N)$.

Clearly, $\pi$ and $\mathcal{P}$ can be built in $O(M \cdot d)$ and $O(N \cdot d)$ time, respectively.

Finally, we show that there exists $a \in A, b \in B$ such that $a \cdot b=0$ iff there is a factor of $w$ that matches $\pi$. Let $a_{i_{1}} \in A$ and $b_{i_{2}} \in B$. Then $a_{i_{1}} \cdot b_{i_{2}}=0$ iff

(i) The factor $\left((x x y y)^{\frac{d}{2}} z\right)^{i_{2}-1}$ of $w$ that precedes $\tilde{F}\left(a_{i_{1}}\right) z$ matches the subexpression " $F\left(b_{1}\right) z \ldots F\left(b_{i_{2}-1}\right) z$ ".

(ii) The factor $\tilde{F}\left(a_{i_{1}}\right) z$ of $w$ matches the subexpression " $F\left(b_{i_{2}}\right) z$ ".

(iii) The factor $\left((x x y y)^{\frac{d}{2}} z\right)^{M-i_{2}}$ of $w$ that succeeds $\tilde{F}\left(a_{i_{1}}\right) z$ matches the subexpression " $F\left(b_{i_{2}+1}\right) z \ldots F\left(b_{M}\right) z$ ". 
It follows from Remark 5.6.1 that there is no algorithm for searching with an homogeneous regular expression of type “.+" working on $O\left((t \cdot s)^{1-\varepsilon}\right)$ time.

Finally, note that if the dimension of the OVP is odd it suffices to replace the factors $\left((x x y y)^{\frac{d}{2}} z\right)^{M-1}$ from $w$ by $\left((x x y y)^{\frac{d-1}{2}} x x z\right)^{M-1}$.

Note that for any homogeneous regular expression of type ".+" of size $s$, we can build in $O(s)$ time an equivalent homogeneous regular expression of type ".*” and size $O(s)$. Therefore, we obtain the following corollary from Theorem 5.6.2.

COROLLARY 5.6.3. There is no algorithm for searching with a regular expression on grammar-compressed text that operates in $O\left((t \cdot s)^{1-\epsilon}\right)$ with $\varepsilon>0$, where $t$ is the size of the compressed text and $s$ is the size of the regular expression, when the expression is homogeneous of type “**”.

THEOREM 5.6.4. There is no algorithm for searching with regular expressions on grammar-compressed text that operates in $O\left((t \cdot s)^{1-\epsilon}\right)$ with $\varepsilon>0$, where $t$ is the size of the compressed text and $s$ is the size of the regular expression, when the expression is homogeneous of type ". I".

Proof. The proof is identical to that of Theorem 5.6.2 but considering the expression

$$
\pi \stackrel{\text { def }}{=} F\left(b_{1}\right) z F\left(b_{2}\right) z \ldots z F\left(b_{M}\right) z "
$$

on the alphabet $\Sigma=\{0,1, z\}$ with

$$
F\left(b_{i}\right)=f\left(b_{i}, 1\right) f\left(b_{i}, 2\right), \ldots, f\left(b_{i}, d\right) \text { and } f(b, j)=\left\{\begin{array}{lll}
0 & \text { if } & (b)_{j}=1 \\
0 \mid 1 & \text { if } & (b)_{j}=0
\end{array}\right.
$$

and the word

$$
w \stackrel{\text { def }}{=}\left(0^{d} z\right)^{M-1} a_{1} z\left(0^{d} z\right)^{M-1} a_{2} z \ldots\left(0^{d} z\right)^{M-1} a_{N} z\left(0^{d} z\right)^{M-1}
$$

Note that, unlike the proof of Theorem 5.6.2, this proof does not depend on the parity of the dimension of the OVP.

\subsubsection{Complexity of Our Implementation}

In the following, we analyze the complexity of the implementation of Algorithm CountLines described in Section 5.3 when the input regular expression is homogeneous of type “.+”, “.*” or “. |" 
As explained in Section 5.3, zearch uses libfa, which applies Thompson's algorithm [Thompson 1968] with on-the-fly $\varepsilon$-removal, to build an NFA for the input regular expression.

However, given a regular expression of size $s$ we can decide in $O(s)$ time whether a expression is homogeneous of type ".+" ".*” or ".|" and, as we show next, use an specialized algorithm for building a DFA with $O(s)$ states in $O(s)$ time for the given expression. Therefore, zearch admits a straightforward modification that allows it to search on grammar-compressed text with homogeneous regular expressions of type ".+", ".*" or ".।" in $O(t \cdot s)$ time, where $s$ is the size of the expression. Next, we show how to build such DFAs from the given regular expressions.

First, observe that every homogeneous regular expression of type ".+" of size $s$ such that it contains no concatenation of the form "a+a+" can be captured by a DFA with $s+1$ states as we show next. Let $a_{1}, \ldots, a_{n}$ be the sequence of letters that appear in an homogeneous expression of type ".+". Then,

$$
\mathcal{D}=\left\langle\left\{q_{i} \mid 0 \leq i \leq n\right\}, \Sigma,\left\{\left(q_{i}, a_{i}, q_{i}\right),\left(q_{i-1}, a_{i}, q_{i}\right) \mid 1 \leq i \leq n\right\},\left\{q_{1}\right\},\left\{q_{n}\right\}\right\rangle
$$

is a DFA for the given expression.

If the expression contains a concatenation of the form " $a+a+$ " then $\mathcal{D}$ is no longer deterministic. In that case, we can replace " $a+a+$ " by "aa+" and, therefore, remove from $\mathcal{D}$ the self-loop corresponding to the first $a$. It is straightforward to check that this change results in a deterministic automaton and it does not alter the generated language, hence it does not alter the result of the search. Figure 5.6 shows the DFA for an homogeneous regular expression of type ".+".

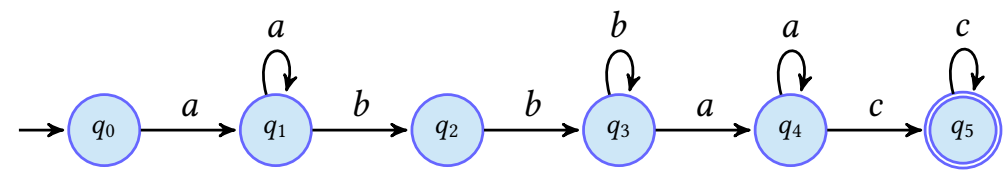

Figure 5.6: DFA for the regular expression " $\mathrm{a}+\mathrm{b}+\mathrm{b}+\mathrm{a}+\mathrm{c}+"=\mathrm{a}+\mathrm{b}+\mathrm{b}+\mathrm{a}+\mathrm{c}+$ ".

On the other hand, let $a_{1}, \ldots, a_{n}$ be the sequence of letters that appear in an homogeneous expression of type “**". For every $a$, let $j_{a}^{k}$ be the smallest index such that $k \leq j_{a}^{k} \leq n$ and $a=a_{j_{a}^{k}}$. Then, the DFA obtained by making every state of $\mathcal{D}$ final and adding the transitions $\left\{\left(q_{i-1}, a_{k}, q_{j_{a_{k}}}\right) \mid 1 \leq i \leq k \leq n\right\}$, is an automaton for the given expression. Note that, if the expression contains a concatenation of the form "a*a*", which will break the determinism of our automata, then we can safely replace it by "a*”. Figure 5.7 shows the DFA for an homogeneous regular expression of type “.*”. 


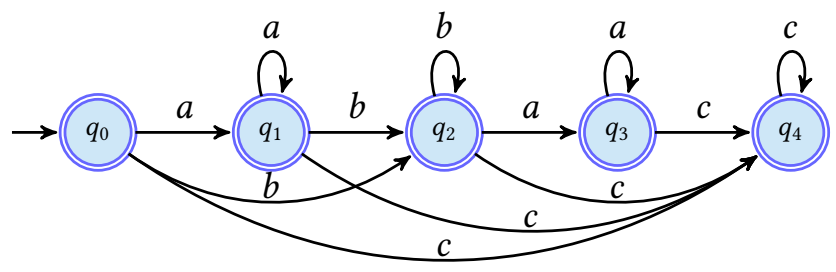

Figure 5.7: DFA for the regular expression " $\mathrm{a} * \mathrm{~b} * \mathrm{~b} * \mathrm{a} * \mathrm{c} *$ " " $\mathrm{a} * \mathrm{~b} * \mathrm{a} * \mathrm{c} *$ ".

Finally, it is straightforward to build a DFA for an homogeneous regular expression of the type ". |" with $n+1$ states where $n$ is the number of concatenations. Figure 5.8 shows the DFA for an homogeneous regular expression of type ". I".

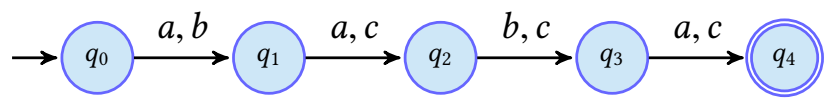

Figure 5.8: DFA for the regular expression " $(\mathrm{a} \mid \mathrm{b})(\mathrm{a} \mid \mathrm{c})(\mathrm{b} \mid \mathrm{c})(\mathrm{a} \mid \mathrm{c})$ ".

It is worth to remark that the DFA of Figure 5.8 is the result of applying Thompson's construction on the input expression. As a consequence, zearch already builds a DFA when the input expression is homogeneous of type ".।" and, therefore, it performs the search in $O(t \cdot s)$.

We conclude that zearch admits a straightforward modification to exhibit $O(t \cdot s)$ time complexity when working on homogeneous regular expressions of types ".+", ".*" and ".|" and, therefore, be nearly optimal for these classes of regular expressions. 



\section{Building Residual Automata}

As shown in Chapter 3, residual automata (RFAs for short) are a class of automata that lies between deterministic (DFAs) and nondeterministic automata (NFAs). They share with DFAs a significant property: the existence of a canonical minimal form for any regular language. On the other hand, they share with NFAs the existence of automata that are exponentially smaller (in the number of states) than the corresponding minimal DFA for the language. These properties make RFAs specially appealing in certain areas of computer science such as Grammatical Inference [Denis et al. 2004; Kasprzik 2011].

RFAs were first introduced by Denis et al. [2000; 2002] who defined an algorithm for residualizing an automaton (see Section 3.2), showed that there exists a unique canonical RFA for every regular language and proved that the residualequivalent of double-reversal method for DFAs [Brzozowski 1962] holds for RFAs, i.e. residualizing an automaton $\mathcal{N}$ whose reverse is residual yields the canonical RFA for $\mathcal{L}(\mathcal{N})$. Later, Tamm [2015] generalized the double-reversal method for RFAs in the same lines as that of Brzozowski and Tamm [2014] for the doublereversal method for DFAs.

The similarities between the determinization and residualization (see Section 3.2) operations and between the double-reversal methods for DFAs and RFAs evidence the existence of a relationship between these two classes of automata. However, the connection between them is not clear and, as a consequence, the relation between the generalization by Brzozowski and Tamm [2014] of the doublereversal method for DFAs and the one by Tamm [2015] for RFAs is not immediate.

In this chapter, we show that quasiorders are fundamental to RFAs as congruences are for DFAs, which evidences the relation between these two classes of automata. To do that, we define a framework of finite-state automata construc- 
tions based on quasiorders over words.

As explained in Chapter 2, Ganty et al. [2019] studied the problem of building DFAs using congruences, i.e., equivalence relations over words with good properties w.r.t. concatenation, and derived several well-known results about minimization of DFAs, including the double-reversal method and its generalization by Brzozowski and Tamm [2014]. While the use of congruences over words suited for the construction of a subclass of residual automata, namely, deterministic automata, these are no longer useful to describe the more general class of nondeterministic residual automata. By moving from congruences to quasiorders, we are able to introduce nondeterminism in our automata constructions.

We consider quasiorders with good properties w.r.t. right and left concatenation. In particular, we define the so-called right language-based quasiorder, whose definition relies on a given regular language; and the right automata-based quasiorder, whose definition relies on a finite representation of the language, i.e., an automaton. We also give counterpart definitions for quasiorders that behave well with respect to left concatenation.

When instantiating our automata constructions using the right languagebased quasiorder, we obtain the canonical RFA for the given language; while using the right automata-based quasiorder yields an RFA for the language generated by the automaton that has, at most, as many states as the RFA obtained by the residualization operation defined by Denis et al. [2002]. Similarly, left automata-based and language-based quasiorders yield co-residual automata, i.e., automata whose reverse is residual.

Our quasiorder-based framework allows us to give a simple correctness proof of the double-reversal method for building the canonical RFA. Moreover, it allows us to generalize this method in the same fashion as Brzozowski and Tamm [2014] generalized the double-reversal method for building the minimal DFA. Specifically, we give a characterization of the class of automata for which our automata-based quasiorder construction yields the canonical RFA.

We compare our characterization with the class of automata, defined by Tamm [2015], for which the residualization operation of Denis et al. [2002] yields the canonical RFA and show that her class of automata is strictly contained in the class we define. Furthermore, we highlight the connection between the generalization of Brzozowski and Tamm [2014] and the one of Tamm [2015] for the double-reversal methods for DFAs and RFAs, respectively.

Finally, we revisit the problem of learning RFAs from a quasiorder-based perspective. Specifically, we observe that the $\mathrm{NL}^{*}$ algorithm defined by Bollig et al. [2009], inspired by the popular Angluin's L* algorithm for learning DFAs [Angluin 1987], can be seen as an algorithm that starts from a quasiorder 
and refines it at each iteration. At the end of each iteration, the automaton built by $\mathrm{NL}^{*}$ coincides with our quasiorder-based automata construction applied to the refined quasiorder.

\subsection{Automata Constructions from Quasiorders}

In this chapter, we consider monotone quasiorders on $\Sigma^{*}$ (and their corresponding closures) and we use them to define RFAs constructions for regular languages. The following lemma gives a characterization of right and left quasiorders.

LЕмма 6.1.1. The following properties hold:

(a) $\leqslant^{r}$ is a right quasiorder iff $\rho_{\leqslant}(u) v \subseteq \rho_{\leqslant}(u v)$, for all $u, v \in \Sigma^{*}$.

(b) $\leqslant^{\ell}$ is a left quasiorder iff $v \rho_{\leqslant \ell}(u) \subseteq \rho_{\leqslant \ell}(v u)$, for all $u, v \in \Sigma^{*}$.

\section{Proof.}

(a) To simplify the notation, we denote $\rho_{\leqslant r}$, the closure induced by $\leqslant^{r}$, by $\rho$.

$(\Rightarrow)$ Let $x \in \rho(v) u$, i.e. $x=\tilde{v} u$ with $v \leqslant^{r} \tilde{v}$. Since $\leqslant^{r}$ is a right quasiorder and $v \leqslant^{r} \tilde{v}$ then $v u \leqslant^{r} \tilde{v} u$. Therefore $x \in \rho(v u)$.

$(\Leftarrow)$ Assume that for each $u, v \in \Sigma^{*}$ and $\tilde{v} \in \rho(v)$ we have that $\tilde{v} u \in$ $\rho(v u)$. Then, $v \leqslant^{r} \tilde{v} \Rightarrow v u \leqslant^{r} \tilde{v} u$.

(b) To simplify the notation we denote $\rho_{\leqslant}$, the closure induced by $\leqslant^{\ell}$, by $\rho$.

$(\Rightarrow)$ Let $x \in u \rho(v)$, i.e. $x=u \tilde{v}$ with $v \leqslant^{\ell} \tilde{v}$. Since $\leqslant^{\ell}$ is a left quasiorder and $v \leqslant^{\ell} \tilde{v}$ then $u v \leqslant^{\ell} u \tilde{v}$. Therefore $x \in \rho(u v)$.

$(\Leftarrow)$ Assume that for each $u, v \in \Sigma^{*}$ and $\tilde{v} \in \rho(v)$ we have that $u \tilde{v} \in$ $\rho(u v)$. Then $v \leqslant^{\ell} \tilde{v} \Rightarrow u v \leqslant^{\ell} u \tilde{v}$.

Given a regular language $L$, we are interested in left and right $L$-consistent quasiorders. We will use the principals of these quasiorders as states of automata constructions that yield RFAs and co-RFAs generating the language $L$. Therefore, in the sequel, we will only consider quasiorders that induce a finite number of principals, i.e., quasiorders $\leqslant$ such that the induced equivalence $\sim \stackrel{\text { def }}{=} \leqslant \cap(\leqslant)^{-1}$ has finite index.

Next, we introduce the notion of L-composite principals which, intuitively, correspond to states of our automata constructions that can be removed without altering the generated language.

Definition 6.1.2 (L-Composite Principal). Let $L$ be a regular language and let $\leqslant^{r}$ (resp. $\leqslant^{\ell}$ ) be a right (resp. left) quasiorder on $\Sigma^{*}$. Given $u \in \Sigma^{*}$, the principal $\rho_{\leqslant}(u)$ 
(resp. $\left.\rho_{\leqslant \ell}(u)\right)$ is L-composite iff

$$
\left.u^{-1} L=\bigcup_{x \in \Sigma^{*}, x<r^{r} u} x^{-1} L \quad \text { (resp. } L u^{-1}=\bigcup_{x \in \Sigma^{*}, x<^{\ell} u} L x^{-1}\right)
$$

If $\rho_{\leqslant r}(u)\left(\right.$ resp. $\left.\rho_{\leqslant}(u)\right)$ is not $L$-composite then it is $L$-prime.

We sometimes use the terms composite and prime principal when the language $L$ is clear from the context. Observe that, if $\rho_{\leqslant r}(u)$ is $L$-composite, for some $u \in \Sigma^{*}$, then so is $\rho_{\leqslant r}(v)$, for every $v \in \Sigma^{*}$ such that $u \sim^{r} v$. The same holds for a left quasiorder $\leqslant \ell$.

Given a regular language $L$ and a right $L$-consistent quasiorder $\leqslant^{r}$, the following automata construction yields an RFA that generates exactly $L$.

Definition 6.1.3 (Automata construction $\mathrm{H}^{r}\left(\leqslant^{r}, L\right)$ ). Let $\leqslant^{r}$ be a right quasiorder and let $L \subseteq \Sigma^{*}$ be a language. Define the automaton $\mathrm{H}^{r}\left(\leqslant^{r}, L\right) \stackrel{\text { def }}{=}\langle Q, \Sigma, \delta, I, F\rangle$ where $Q=\left\{\rho_{\leqslant r}(u) \mid u \in \Sigma^{*}, \rho_{\leqslant r}(u)\right.$ is L-prime $\}, I=\left\{\rho_{\leqslant r}(u) \in Q \mid \varepsilon \in \rho_{\leqslant r}(u)\right\}$, $F=\left\{\rho_{\leqslant r}(u) \in Q \mid u \in L\right\}$ and $\delta\left(\rho_{\leqslant r}(u), a\right)=\left\{\rho_{\leqslant r}(v) \in Q \mid \rho_{\leqslant r}(u) \cdot a \subseteq \rho_{\leqslant r}(v)\right\}$ for all $\rho_{\leqslant r}(u) \in Q, a \in \Sigma$.

LЕммА 6.1.4. Let $L \subseteq \Sigma^{*}$ be a regular language and let $\leqslant^{r}$ be a right $L$-consistent quasiorder. Then, $\mathrm{H}^{r}\left(\leqslant^{r}, L\right)$ is an RFA such that $\mathcal{L}\left(\mathrm{H}^{r}\left(\leqslant^{r}, L\right)\right)=L$.

Proof. To simplify the notation, we denote $\rho_{\leqslant r}$, the closure induced by the quasiorder $\leqslant^{r}$, simply by $\rho$. Let $\mathcal{H}=\mathrm{H}^{r}\left(\leqslant^{r}, L\right)=\langle Q, \Sigma, \delta, I, F\rangle$. We first show that $\mathcal{H}$ is an RFA, i.e.

$$
W_{\rho(u), F}^{\mathcal{H}}=u^{-1} L, \quad \text { for each } \rho(u) \in Q .
$$

Let us prove that $w \in u^{-1} L \Rightarrow w \in W_{\rho(u), F}^{\mathcal{H}}$. We proceed by induction on the length of $w$.

- Base case: Assume $w=\varepsilon$. Then,

$$
\varepsilon \in u^{-1} L \Rightarrow u \in L \Rightarrow \rho(u) \in F \Rightarrow \varepsilon \in W_{\rho(u), F}^{\mathcal{H}} .
$$

- Inductive step: Assume that the hypothesis holds for each word $x \in \Sigma^{*}$ with $|x| \leq n$, where $n \geq 1$, and let $w \in \Sigma^{*}$ be such that $|w|=n+1$. Then $w=a x$ with $|x|=n$ and $a \in \Sigma$.

$$
\begin{aligned}
a x \in u^{-1} L & \Rightarrow \text { [By definition of quotient] } \\
x \in(u a)^{-1} L & \Rightarrow
\end{aligned}
$$


[By Def. 6.1.2, $\rho(u a)$ is $L$-prime (so $z \stackrel{\text { def }}{=} u a$ ) or $(u a)^{-1} L=\bigcup_{x_{i}<r} x_{i} x_{i}^{-1} L$ (so $z \stackrel{\text { def }}{=} x_{i}$ )]

$$
\begin{array}{ccc}
\exists \rho(z) \in Q, x \in z^{-1} L \wedge \rho(u a) \subseteq \rho(z) & \Rightarrow & \text { [By I.H., Lemma 6.1.1 and Def. 6.1.3] } \\
x \in W_{\rho(z), F}^{\mathcal{H}} \wedge \rho(z) \in \delta(\rho(u), a) & \Rightarrow & {\left[\text { By definition of } W_{S, T}\right]} \\
a x \in W_{\rho(u), F}^{\mathcal{H}} & &
\end{array}
$$

We now prove the other side of the implication, $w \in W_{\rho(u), F}^{\mathcal{H}} \Rightarrow w \in u^{-1} L$.

- Base case: Let $w=\varepsilon$. By Definition 6.1.3,

$$
\varepsilon \in W_{\rho(u), F}^{\mathcal{H}} \Rightarrow \exists \rho(x) \in Q, x \in L \wedge \rho(u) \varepsilon \subseteq \rho(x) .
$$

Since $\rho(L)=L$, we have that $u \varepsilon \in L$, hence $\varepsilon \in u^{-1} L$.

- Inductive step: Assume the hypothesis holds for each $x \in \Sigma^{*}$ with $|x| \leq n$, where $n \geq 1$, and let $w \in \Sigma^{*}$ be such that $|w|=n+1$. Then $w=a x$ with $|x|=n$ and $a \in \Sigma$.

$$
\begin{array}{rll}
a x \in W_{\rho(u), F}^{\mathcal{H}} & \Rightarrow & {[\text { By Definition 6.1.3] }} \\
x \in W_{\rho(y), F}^{\mathcal{H}} \wedge \rho(u) a \subseteq \rho(y) \Rightarrow & {\left[\text { By I.H. and since } \rho \text { is induced by } \leqslant^{r}\right. \text { ] }} \\
x \in y^{-1} L \wedge y \leqslant^{r} u a \Rightarrow & {[\text { By de Luca and Varricchio [1994]] }} \\
x \in y^{-1} L \wedge y^{-1} L \subseteq(u a)^{-1} L \Rightarrow & {\left[\text { Since } x \in(u a)^{-1} L \Rightarrow a x \in u^{-1} L\right]} \\
a x \in u^{-1} L . &
\end{array}
$$

We have shown that $\mathcal{H}$ is an RFA. Finally, we show that $\mathcal{L}(\mathcal{H})=L$. First note that

$$
\mathcal{L}(\mathcal{H})=\bigcup_{\rho(u) \in I} W_{\rho(u), F}^{\mathcal{H}}=\bigcup_{\rho(u) \in I} u^{-1} L
$$

where the first equality holds by definition of $\mathcal{L}(\mathcal{H})$ and the second by Equation (6.1). On one hand, we have that $\bigcup_{\rho(u) \in I} u^{-1} L \subseteq L$ since, by Definition 6.1.3, $\varepsilon \in \rho(u)$ for each $\rho(u) \in I$, hence $u \leqslant^{r} \varepsilon$ which, as shown by de Luca and Varricchio [1994], implies that $u^{-1} L \subseteq \varepsilon^{-1} L=L$. Then,

Let us now show that $L \subseteq \bigcup_{\rho(u) \in I} u^{-1} L$. First, let us assume that $\rho(\varepsilon) \in I$.

$$
L=\varepsilon^{-1} L \subseteq \bigcup_{\rho(u) \in I} u^{-1} L
$$


Now suppose that $\rho(\varepsilon) \notin I$, i.e. $\rho(\varepsilon)$ is $L$-composite. Then,

$$
L=\varepsilon^{-1} L=\bigcup_{u r^{r} \varepsilon} u^{-1} L=\bigcup_{\rho(u) \in I} u^{-1} L .
$$

where the last equality follows from $\rho(u) \in I \Leftrightarrow \varepsilon \in \rho(u)$.

Given a regular language $L$ and a left $L$-consistent quasiorder $\leqslant^{\ell}$, we can give a similar automata construction of a co-RFA that recognizes exactly $L$

Definition 6.1.5 (Automata construction $\mathrm{H}^{\ell}\left(\leqslant^{\ell}, L\right)$ ). Let $\leqslant^{\ell}$ be a left quasiorder and let $L \subseteq \Sigma^{*}$ be a language. Define the automaton $\mathrm{H}^{\ell}\left(\leqslant^{\ell}, L\right) \stackrel{\text { def }}{=}\langle Q, \Sigma, \delta, I, F\rangle$ where $Q=\left\{\rho_{\leqslant \ell}(u) \mid u \in \Sigma^{*}, \rho_{\leqslant \ell}(u)\right.$ is L-prime $\}, I=\left\{\rho_{\leqslant^{\ell}}(u) \in Q \mid u \in L\right\}, F=$ $\left\{\rho_{\leqslant^{\ell}}(u) \in Q \mid \varepsilon \in \rho_{\leqslant^{\ell}}(u)\right\}$, and $\delta\left(\rho_{\leqslant^{\ell}}(u), a\right)=\left\{\rho_{\leqslant \ell}(v) \in Q \mid a \cdot \rho_{\leqslant^{\ell}}(v) \subseteq \rho_{\leqslant \ell}(u)\right\}$ for all $\rho_{\leqslant \ell}(u) \in Q, a \in \Sigma$.

LEMMA 6.1.6. Let $L \subseteq \Sigma^{*}$ be a language and let $\leqslant^{\ell}$ be a left $L$-consistent quasiorder. Then $\mathrm{H}^{\ell}\left(\leqslant^{\ell}, L\right)$ is a co-RFA such that $\mathcal{L}\left(\mathrm{H}^{\ell}\left(\leqslant^{\ell}, L\right)\right)=L$.

Proof. To simplify the notation we denote $\rho_{\leqslant}$, the closure induced by the quasiorder $\leqslant^{\ell}$, simply by $\rho$. Let $\mathcal{H}=\mathrm{H}^{\ell}\left(\leqslant^{\ell}, L\right)=\langle Q, \Sigma, \delta, I, F\rangle$. We first show that $\mathcal{H}$ is a co-RFA.

$$
W_{I, \rho(u)}^{\mathcal{H}}=L u^{-1}, \quad \text { for each } \rho(u) \in Q .
$$

Let us prove that $w \in L u^{-1} \Rightarrow w \in W_{I, \rho(u)}^{\mathcal{H}}$. We proceed by induction.

- Base case: Let $w=\varepsilon$. Then

$$
\varepsilon \in L u^{-1} \Rightarrow u \in L \Rightarrow \rho(u) \in I \Rightarrow \varepsilon \in W_{I, \rho(u)}^{\mathcal{H}} .
$$

- Inductive step: Assume the hypothesis holds for all $x \in \Sigma^{*}$ with $|x| \leq n$, where $n \geq 1$, and let $w \in \Sigma^{*}$ be such that $|w|=n+1$. Then $w=x a$ with $|x|=n$ and $a \in \Sigma$.

$$
\begin{aligned}
x a \in L u^{-1} & \Rightarrow \quad[\text { By definition of quotient }] \\
x \in L(a u)^{-1} & \Rightarrow
\end{aligned}
$$

[By Def. 6.1.2, $\rho(u a)$ is $L$-prime (so $z \stackrel{\text { def }}{=} a u$ ) or $L(a u)^{-1}=\bigcup_{x_{i}<^{\ell} a u} L x_{i}^{-1}$ (so $z \stackrel{\text { def }}{=} x_{i}$ )] $\exists \rho(z) \in Q, x \in L z^{-1} \wedge \rho(a u) \subseteq \rho(z) \Rightarrow \quad$ [By I.H., Lemma 6.1.1 and Def. 6.1.5] 


$$
\begin{gathered}
x \in W_{I, \rho(z)}^{\mathcal{H}} \wedge \rho(u) \in \delta(\rho(z), a) \Rightarrow \quad\left[\text { By definition of } W_{S, T}\right] \\
x a \in W_{I, \rho(u)}^{\mathcal{H}} .
\end{gathered}
$$

We now prove the other side of the implication, $w \in W_{I, \rho(u)}^{\mathcal{H}} \Rightarrow w \in L u^{-1}$.

- Base case: Let $w=\varepsilon$. Then

$$
\varepsilon \in W_{I, \rho(u)}^{\mathcal{H}} \Rightarrow \exists \rho(x) \in Q, x \in L \wedge \varepsilon \rho(u) \subseteq \rho(x) .
$$

Since $\rho(L)=L$, we have that $\varepsilon u \in L$, hence $\varepsilon \in L u^{-1}$.

- Inductive step: Assume the hypothesis holds for each $x \in \Sigma^{*}$ with $|x| \leq n$, where $n \geq 1$, and let $w \in \Sigma^{*}$ be such that $|w|=n+1$. Then $w=x \cdot a$ with $|x|=n$ and $a \in \Sigma$.

$$
\begin{array}{rll}
x a \in W_{I, \rho(u)}^{\mathcal{H}} \Rightarrow & {[\text { By Definition 6.1.5] }} \\
a \cdot \rho(u) \subseteq \rho(y) \wedge x \in W_{I, \rho(y)}^{\mathcal{H}} \Rightarrow & {\left[\text { By I.H. and since } \rho \text { is induced by } \leqslant^{\ell}\right]} \\
y \leqslant^{\ell} a u \wedge x \in L y^{-1} \Rightarrow & {[\text { By de Luca and Varricchio [1994]] }} \\
L y^{-1} \subseteq L(a u)^{-1} \wedge x \in L y^{-1} \Rightarrow & {\left[\text { Since } x \in L(a u)^{-1} \Rightarrow x a \in L u^{-1}\right]} \\
x a \in u^{-1} L . &
\end{array}
$$

We have shown that $\mathcal{H}$ is a co-RFA. Finally, we show that $\mathcal{L}(\mathcal{H})=L$. First note that

$$
\mathcal{L}(\mathcal{H})=\bigcup_{\rho(u) \in F} W_{I, \rho(u)}^{\mathcal{H}}=\bigcup_{\rho(u) \in F} L u^{-1},
$$

where the first equality holds by definition of $\mathcal{L}(\mathcal{H})$ and the second by Equation (6.2). On one hand, we have that $\bigcup_{\rho(u) \in F} L u^{-1} \subseteq L$ since, by Definition 6.1.5, $\varepsilon \in \rho(u)$ for each $\rho(u) \in F$, hence $u \leqslant^{\ell} \varepsilon$ which, as shown by de Luca and Varricchio [1994], implies that $L u^{-1} \subseteq L \varepsilon^{-1}=L$.

Let us now show that $L \subseteq \bigcup_{\rho(u) \in F} L u^{-1}$. First, let us assume that $\rho(\varepsilon) \in F$. Then,

$$
L=L \varepsilon^{-1} \subseteq \bigcup_{\rho(u) \in F} L u^{-1} .
$$

Now suppose that $\rho(\varepsilon) \notin F$, i.e. $\rho(\varepsilon)$ is $L$-composite. Then,

$$
L=L \varepsilon^{-1}=\bigcup_{u<^{\ell} \varepsilon} L u^{-1}=\bigcup_{\rho(u) \in F} u^{-1} L .
$$

where the last equality follows from $\rho(u) \in F \Leftrightarrow \varepsilon \in \rho(u)$. 
Observe that the automaton $\mathcal{H}^{r}=\mathrm{H}^{r}\left(\leqslant^{r}, L\right)\left(\right.$ resp. $\left.\mathcal{H}^{\ell}=\mathrm{H}^{\ell}\left(\leqslant^{\ell}, L\right)\right)$ is finite, since we assume $\leqslant^{r}$ (resp. $\leqslant^{\ell}$ ) induces a finite number of principals. Note also that $\mathcal{H}^{r}$ (resp. $\mathcal{H}^{\ell}$ ) possibly contains empty (resp. unreachable) states but no state is unreachable (resp. empty).

Moreover, notice that by keeping all principals of $\leqslant^{r}$ (resp. $\left.\leqslant^{\ell}\right)$ as states, instead of only the $L$-prime ones as in Definition 6.1.3 (resp. Definition 6.1.5), we would obtain an RFA (resp. a co-RFA) with (possibly) more states that also recognizes $L$.

Finally, Lemma 6.1.7 shows that $\mathcal{H}^{\ell}$ and $\mathcal{H}^{r}$ inherit the left-right duality between $\leqslant^{\ell}$ and $\leqslant^{r}$ through the reverse operation.

Lемма 6.1.7. Let $\leqslant^{r}$ and $\leqslant^{\ell}$ be a right and a left quasiorder, respectively, and let $L \subseteq \Sigma^{*}$ be a language. If the following property holds

$$
u \leqslant^{r} v \Leftrightarrow u^{R} \leqslant^{\ell} v^{R}
$$

then $\mathrm{H}^{r}\left(\leqslant^{r}, L\right)$ is isomorphic to $\left(\mathrm{H}^{\ell}\left(\leqslant^{\ell}, L^{R}\right)\right)^{R}$.

Proof. Let $\mathrm{H}^{r}\left(\leqslant^{r}, L\right)=\langle Q, \Sigma, \delta, I, F\rangle$ and $\left(\mathrm{H}^{\ell}\left(\leqslant^{\ell}, L^{R}\right)\right)^{R}=\langle\widetilde{Q}, \Sigma, \widetilde{\delta}, \widetilde{I}, \widetilde{F}\rangle$. We will show that $\mathrm{H}^{r}\left(\leqslant^{r}, L\right)$ is isomorphic to $\left(\mathrm{H}^{\ell}\left(\leqslant^{\ell}, L^{R}\right)\right)^{R}$.

Let $\varphi: Q \rightarrow \widetilde{Q}$ be a mapping assigning to each state $\rho_{\leqslant r}(u) \in Q$ with $u \in \Sigma^{*}$, the state $\rho_{\leqslant \ell}\left(u^{R}\right) \in \widetilde{Q}$. Next, we show that $\varphi$ is an NFA isomorphism between $\mathrm{H}^{r}\left(\leqslant^{r}, L\right)$ and $\left(\mathrm{H}^{\ell}\left(\leqslant^{\ell}, L^{R}\right)\right)^{R}$.

Observe that:

$$
\begin{aligned}
u^{-1} L=\bigcup_{x<r^{r} u} x^{-1} L \Leftrightarrow & {\left[\text { Since }\left(\bigcup S_{i}\right)^{R}=\bigcup S_{i}^{R}\right] } \\
\left(u^{-1} L\right)^{R}=\bigcup_{x<^{r} u}\left(x^{-1} L\right)^{R} \Leftrightarrow & {\left[\text { Since }\left(u^{-1} L\right)^{R}=L^{R}\left(u^{R}\right)^{-1}\right] } \\
L^{R}\left(u^{R}\right)^{-1}=\bigcup_{x<r^{r} u} L^{R}\left(x^{R}\right)^{-1} \Leftrightarrow & \text { [By Equation (6.3)] } \\
L^{R}\left(u^{R}\right)^{-1}=\bigcup_{x^{R}<^{\ell} u^{R}} L^{R}\left(x^{R}\right)^{-1} . &
\end{aligned}
$$

Therefore $\rho_{\leqslant}(u)$ is $L$-composite iff $\rho_{\leqslant}\left(u^{R}\right)$ is $L^{R}$-composite, hence $\varphi(Q)=\widetilde{Q}$.

Since

$$
\varepsilon \in \rho_{\leqslant}(u) \Leftrightarrow u \leqslant^{r} \varepsilon \Leftrightarrow u^{r} \leqslant^{\ell} \varepsilon \Leftrightarrow \varepsilon \in \rho_{\leqslant \ell}\left(u^{R}\right),
$$

we have that $\rho_{\leqslant r}(u)$ is an initial state of $\mathrm{H}^{r}\left(\leqslant^{r}, L\right)$ iff $\rho_{\leqslant \ell}\left(u^{R}\right)$ is a final state of $\mathrm{H}^{\ell}\left(\leqslant^{\ell}, L^{R}\right)$, i.e. an initial state of $\left(\mathrm{H}^{\ell}\left(\leqslant^{\ell}, L^{R}\right)\right)^{R}$. Therefore, $\varphi(I)=\widetilde{I}$. 
Since

$$
\rho_{\leqslant}(u) \subseteq L \Leftrightarrow u \in L \Leftrightarrow u^{r} \in L^{R},
$$

we have that $\rho_{\leqslant r}(u)$ is a final state of $\mathrm{H}^{r}\left(\leqslant^{r}, L\right)$ iff $\rho_{\leqslant \ell}\left(u^{R}\right)$ is an initial state of $\mathrm{H}^{\ell}\left(\leqslant^{\ell}, L^{R}\right)$, i.e. a final state of $\left(\mathrm{H}^{\ell}\left(\leqslant^{\ell}, L^{R}\right)\right)^{R}$. Therefore, $\varphi(F)=\widetilde{F}$.

It remains to show that $q^{\prime} \in \delta(q, a) \Leftrightarrow \varphi\left(q^{\prime}\right) \in \widetilde{\delta}(\varphi(q), a)$, for all $q, q^{\prime} \in Q$ and $a \in \sum$. Assume that $q=\rho_{\leqslant r}(u)$ for some $u \in \Sigma^{*}, q^{\prime}=\rho_{\leqslant r}(v)$ for some $v \in \Sigma^{*}$ and $q^{\prime} \in \delta(q, a)$ with $a \in \Sigma$. Then,

$$
\begin{aligned}
\rho_{\leqslant r}(v) \in \delta\left(\rho_{\leqslant r}(u), a\right) & \Leftrightarrow & {[\text { By Definition 6.1.3] }} \\
\rho_{\leqslant r}(u) a \subseteq \rho_{\leqslant r}(v) & \Leftrightarrow & {\left[\text { By definition of } \rho_{\leqslant r}\right. \text { and Lemma 6.1.1] }} \\
v \leqslant^{r} u a \Leftrightarrow & & {\left[\text { By Equation }(6.3) \text { and }(u a)^{R}=a u^{R}\right] } \\
v^{r} \leqslant^{\ell} a u^{R} & \Leftrightarrow & {\left[\text { By definition of } \rho_{\leqslant \ell}\right. \text { and Lemma 6.1.1] }} \\
a \rho_{\leqslant \ell}\left(u^{R}\right) \subseteq \rho_{\leqslant \ell}\left(v^{R}\right) & \Leftrightarrow & \text { [By Definition 6.1.5] } \\
\rho_{\leqslant \ell}\left(v^{R}\right) \in \widetilde{\delta}\left(\rho_{\leqslant \ell}\left(u^{R}\right), a\right) & \Leftrightarrow & {\left[\text { Definition of } q, q^{\prime} \text { and } \varphi\right] } \\
\varphi\left(q^{\prime}\right) \in \widetilde{\delta}(\varphi(q), a) . & &
\end{aligned}
$$

\subsubsection{On the Size of $\mathrm{H}^{r}\left(\leqslant^{r}, L\right)$ and $\mathrm{H}^{\ell}\left(\leqslant^{\ell}, L\right)$}

We conclude this section with a note on the sizes of the automata constructions $\mathrm{H}^{r}\left(\leqslant^{r}, L\right)$ and $\mathrm{H}^{\ell}\left(\leqslant^{\ell}, L\right)$ when applied to quasiorders satisfying $\leqslant_{1}^{r} \subseteq \leqslant_{2}^{r}$ and $\leqslant_{1}^{\ell} \subseteq \leqslant_{2}^{\ell}$, respectively.

The following result establishes a relationship between the $L$-composite principals for two comparable right quasiorders $\leqslant_{1}^{r} \subseteq \leqslant_{2}^{r}$. This result is then used in Theorem 6.1.9 to show that the number of $L$-prime principals induced by $\leqslant_{1}^{r}$ is greater or equal than the number number of $L$-prime principals induced by $\leqslant_{2}^{r}$.

As a consequence, if $\leqslant_{1}^{r} \subseteq \leqslant_{2}^{r}$ then the automaton $\mathrm{H}^{r}\left(\leqslant_{1}^{r}, L\right)$ has, at least, as many states as $\mathrm{H}^{r}\left(\leqslant_{2}^{r}, L\right)$. The same holds for left quasiorders and $\mathrm{H}^{\ell}$.

LЕмМА 6.1.8. Let $L \subseteq \Sigma^{*}$ be a regular language and let $u \in \Sigma^{*}$. Let $\leqslant_{1}^{r}$ and $\leqslant_{2}^{r}$ be two L-consistent right quasiorders such that $\leqslant_{1}^{r} \subseteq \leqslant_{2}^{r}$. Then

$\rho_{\leqslant_{1}^{r}}(u)$ is L-composite $\Rightarrow\left(\rho_{\leqslant_{2}^{r}}(u)\right.$ is L-composite $\left.\vee \exists x<_{1}^{r} u, \rho_{\leqslant_{2}^{r}}(u)=\rho_{\leqslant_{2}^{r}}(x)\right)$.

Similarly holds for left quasiorders. 
Proof. Let $u \in \Sigma^{*}$ be such that $\rho_{\leqslant 1}(u)$ is $L$-composite. Then we have that $u^{-1} L=\bigcup_{x \in \Sigma^{*}, x<{ }_{1}^{r} u} x^{-1} L$. On the other hand, since $\leqslant_{2}^{r}$ is a right $L$-consistent quasiorder, we have that $\leqslant_{2}^{r} \subseteq \leqslant_{L}^{r}$, as shown by de Luca and Varricchio [1994]. Therefore $u^{-1} L \supseteq \bigcup_{x \in \Sigma^{*}, x<{ }_{2}^{r} u} x^{-1} L$. There are now two possibilities:

- For all $x \in \Sigma^{*}$ such that $x<{ }_{1}^{r} u$ we have that $x<{ }_{2}^{r} u$. In that case we have that $u^{-1} L=\bigcup_{x \in \Sigma^{*}, x<{ }_{2}^{r} u} x^{-1} L$, hence $\rho_{\leqslant_{2}^{r}}(u)$ is $L$-composite.

- There exists $x \in \Sigma^{*}$ such that $x<_{1}^{r} u$, hence $x \leqslant_{2}^{r} u$, but $x \nless_{2}^{r} u$. In that case, it follows that $\rho_{\leqslant_{2}^{r}}(x)=\rho_{\leqslant_{2}^{r}}(u)$.

The proof for left quasiorders is symmetric.

THEOREM 6.1.9. Let $L \subseteq \Sigma^{*}$ and let $\leqslant_{1}$ and $\leqslant_{2}$ be two right or two left $L$-consistent quasiorders such that $\leqslant_{1} \subseteq \leqslant_{2}$. Then

$\mid\left\{\rho_{\leqslant_{1}}(u) \mid u \in \Sigma^{*} \wedge \rho_{\leqslant_{1}}(u)\right.$ is L-prime $\}|\geq|\left\{\rho_{\leqslant_{2}}(u) \mid u \in \Sigma^{*} \wedge \rho_{\leqslant_{2}}(u)\right.$ is L-prime $\} \mid$

Proof. We proceed by showing that for every $L$-prime $\rho_{\leqslant_{2}}(u)$ there exists an $L$-prime $\rho_{\leqslant_{1}}(x)$ such that $\rho_{\leqslant_{2}}(x)=\rho_{\leqslant_{2}}(u)$. Clearly, this entails that there are, at least, as many $L$-prime principals for $\leqslant_{1}$ as there are for $\leqslant_{2}$.

Let $\rho_{\leqslant_{2}}(u)$ be $L$-prime.

If $\rho_{\leqslant_{1}}(u)$ is $L$-prime, we are done. Otherwise, by Lemma 6.1.8, we have that there exists $x<_{1} u$ such that $\rho_{\leqslant_{2}}(u)=\rho_{\leqslant_{2}}(x)$.

We repeat the reasoning with $x$. If $\rho_{\leqslant_{1}}(x)$ is $L$-prime, we are done. Otherwise, there exists $x_{1}<_{1} x$ such that $\rho_{\leqslant_{2}}(u)=\rho_{\leqslant_{2}}(x)=\rho_{\leqslant_{2}}\left(x_{1}\right)$.

Since $\leqslant_{1}$ induces finitely many principals, there are no infinite strictly descending chains and, therefore, there exists $x_{n}$ such that $\rho_{\leqslant_{2}}(u)=\rho_{\leqslant_{2}}(x)=$ $\rho_{\leqslant_{2}}\left(x_{1}\right)=\ldots=\rho_{\leqslant_{2}}\left(x_{n}\right)$ and $\rho_{\leqslant_{1}}\left(x_{n}\right)$ is $L$-prime.

\subsection{Language-based Quasiorders and their Approximation using NFAs}

In this section we instantiate our automata constructions using two classes of quasiorders, namely, the so-called Nerode's quasiorders [de Luca and Varricchio 1994], whose definition is based on a given regular language; and the automatabased quasiorders, whose definition is based on a finite representation of the language, i.e, an automaton.

Both quasiorders have been used previously in Chapter 4 in order to derive algorithms for solving the language inclusion problem between regular languages. 
We recall their definitions next:

$$
\begin{array}{lr}
u \leqslant_{L}^{r} v \stackrel{\text { def }}{\Leftrightarrow} u^{-1} L \subseteq v^{-1} L & \text { Right-language-based Quasiorder } \\
u \leqslant_{L}^{\ell} v \stackrel{\text { def }}{\Leftrightarrow} L u^{-1} \subseteq L v^{-1} & \text { Left-language-based Quasiorder } \\
u \leqslant_{\mathcal{N}}^{r} v \stackrel{\text { def }}{\Leftrightarrow} \operatorname{post}_{u}^{\mathcal{N}}(I) \subseteq \operatorname{post}_{v}^{\mathcal{N}}(I) & \text { Right-Automata-based Quasiorder } \\
u \leqslant_{\mathcal{N}}^{\ell} v \stackrel{\text { def }}{\Leftrightarrow} \operatorname{pre}_{u}^{\mathcal{N}}(F) \subseteq \operatorname{pre}_{v}^{\mathcal{N}}(F) & \text { Left-Automata-based Quasiorder }
\end{array}
$$

As explained in Chapter 4, de Luca and Varricchio [2011] showed that for every regular language $L$ there exists a finite number of quotients $u^{-1} L$ and, therefore, $\leqslant_{L}^{r}$ and $\leqslant_{L}^{\ell}$ are well-quasiorders. On the other hand, the automatabased quasiorders are also well-quasiorders. Therefore, all the quasiorders defined above induce a finite number of principals.

Remark 6.2.1. The pairs of quasiorders $\leqslant_{L}^{r}-\leqslant_{L}^{\ell}$ and $\leqslant_{\mathcal{N}}^{r}-\leqslant_{\mathcal{N}}^{\ell}$ are dual, i.e.

$$
u \leqslant_{L}^{r} v \Leftrightarrow u^{R} \leqslant_{L}^{\ell} v^{R} \text { and } u \leqslant_{\mathcal{N}}^{r} v \Leftrightarrow u^{R} \leqslant_{\mathcal{N}}^{\ell} v^{R} \text {. }
$$

The following result shows that the principals of $\leqslant_{\mathcal{N}}^{r}$ and $\leqslant_{\mathcal{N}}^{\ell}$ can be described, respectively, as intersections of left and right languages of the states of $\mathcal{N}$ while the principals of $\leqslant_{L}^{r}$ and $\leqslant_{L}^{\ell}$ can be described as intersections of left and right quotients of $L$.

LEMMA 6.2.2. Let $\mathcal{N}=\langle Q, \Sigma, \delta, I, F\rangle$ be an NFA with $\mathcal{L}(\mathcal{N})=L$. Then, for every $u \in \Sigma^{*}$,

$$
\begin{array}{ll}
\rho_{\leqslant_{\mathcal{N}}^{r}}(u)=\bigcap_{q \in \operatorname{post}_{u}^{\mathcal{N}}(I)} W_{I, q}^{\mathcal{N}} & \rho_{\leqslant_{L}^{r}}(u)=\bigcap_{w \in \Sigma^{*}, w \in u^{-1} L} L w^{-1} \\
\rho_{\leqslant_{\mathcal{N}}^{\ell}}(u)=\bigcap_{q \in \operatorname{pre}_{u}^{\mathcal{N}}(I)} W_{q, F}^{\mathcal{N}} & \rho_{\leqslant_{L}^{\ell}}(u)=\bigcap_{w \in \Sigma^{*}, w \in L u^{-1} L^{-1} L .}
\end{array}
$$

Proof. We prove the lemma for the principals induced by $\leqslant_{L}^{r}$ and $\leqslant_{\mathcal{N}}^{r}$. The proofs for the left quasiorders are symmetric.

For each $u \in \Sigma^{*}$ we have that

$$
\begin{aligned}
\rho_{\leqslant_{\mathcal{N}}^{r}}(u)= & {\left[\text { By definition of } \rho_{\leqslant_{\mathcal{N}}^{r}}\right] } \\
\left\{v \in \Sigma^{*} \mid \operatorname{post}_{u}^{\mathcal{N}}(I) \subseteq \operatorname{post}_{v}^{\mathcal{N}}(I)\right\}= & \text { [By definition of set inclusion] } \\
\left\{v \in \Sigma^{*} \mid \forall q \in \operatorname{post}_{u}^{\mathcal{N}}(I), q \in \operatorname{post}_{v}^{\mathcal{N}}(I)\right\}= & {\left[\text { Since } q \in \operatorname{post}_{v}^{\mathcal{N}}(I) \Leftrightarrow v \in W_{I, q}^{\mathcal{N}}\right] } \\
\left\{v \in \Sigma^{*} \mid \forall q \in \operatorname{post}_{u}^{\mathcal{N}}(I), v \in W_{I, q}^{\mathcal{N}}\right\}= & \text { [By definition of intersection] } \\
\bigcap\left\{\operatorname{post}_{u}^{\mathcal{N}(I)} W_{I, q}^{\mathcal{N}} .\right. &
\end{aligned}
$$


On the other hand,

$$
v \in \bigcap_{w \in \Sigma^{*}, w \in u^{-1} L} L w^{-1} \Leftrightarrow \quad \text { [By definition of intersection] }
$$

$\forall w \in \Sigma^{*}, w \in u^{-1} L \Rightarrow v \in L w^{-1} \Leftrightarrow \quad\left[\right.$ Since $\left.\forall x, y \in \Sigma^{*}, x \in L y^{-1} \Leftrightarrow y \in x^{-1} L\right]$

$\forall w \in \Sigma^{*}, w \in u^{-1} L \Rightarrow w \in v^{-1} L \Leftrightarrow \quad$ [By definition of set inclusion]

$$
\begin{aligned}
& \left.u^{-1} L \subseteq v^{-1} L \Leftrightarrow \quad \text { [By definition of } \rho_{\leqslant_{L}^{\ell}}(u)\right] \\
& v \in \rho_{\leqslant_{L}^{r}}(u)
\end{aligned}
$$

As shown by Lemma 4.3.7, given an NFA $\mathcal{N}$ with $L=\mathcal{L}(\mathcal{N})$, the quasiorders $\leqslant_{L}^{r}$ and $\leqslant_{\mathcal{N}}^{r}$ are right $L$-consistent, while the quasiorders $\leqslant_{L}^{\ell}$ and $\leqslant_{\mathcal{N}}^{\ell}$ are left $L$ consistent. Therefore, by Lemma 6.1.4 and 6.1.6, our automata constructions applied to these quasiorders yield automata for $L$.

Finally, recall that, as shown by de Luca and Varricchio [1994], $\leqslant_{\mathcal{N}}^{r}$ is finer than $\leqslant_{L}^{r}$, i.e., $\leqslant_{\mathcal{N}}^{r} \subseteq \leqslant_{L}^{r}$. In that sense we say $\leqslant_{\mathcal{N}}^{r}$ approximates $\leqslant_{L}^{r}$. As the following lemma shows, the approximation is precise, i.e., $\leqslant_{\mathcal{N}}^{r}=\leqslant_{L}^{r}$, whenever $\mathcal{N}$ is a co-RFA.

LEMMA 6.2.3. Let $\mathcal{N}=\langle Q, \Sigma, \delta, I, F\rangle$ be a co-RFA with $L=\mathcal{L}(\mathcal{N})$. Then $\leqslant_{L}^{r}=\leqslant_{\mathcal{N}}^{r}$. Similarly, if $\mathcal{N}$ is an RFA then $\leqslant_{L}^{\ell}=\leqslant_{\mathcal{N}}^{\ell}$

Proof. It is straightforward to check that the following implication holds for every NFA $\mathcal{N}$ and $u, v \in \Sigma^{*}$.

$$
\operatorname{post}_{u}^{\mathcal{N}}(I) \subseteq \operatorname{post}_{v}^{\mathcal{N}}(I) \Rightarrow W_{\operatorname{post}_{u}^{\mathcal{N}}(I), F}^{\mathcal{N}} \subseteq W_{\operatorname{post}_{v}^{\mathcal{N}}(I), F}^{\mathcal{N}}
$$

Next we show that the reverse implication also holds when $\mathcal{N}$ is a co-RFA. Let $u, v \in \Sigma^{*}$ be such that $W_{\operatorname{post}_{u}^{\mathcal{N}}(I), F}^{\mathcal{N}} \subseteq W_{\operatorname{post}_{v}^{\mathcal{N}}(I), F}^{\mathcal{N}}$. Then,

$$
\begin{aligned}
& q \in \operatorname{post}_{u}^{\mathcal{N}}(I) \Rightarrow \quad[\text { Since } \mathcal{N} \text { is co-RFA] } \\
& \exists x \in \Sigma^{*}, u \in W_{I, q}=L x^{-1} \Rightarrow \quad\left[\text { Since } u \in L x^{-1} \Rightarrow x \in u^{-1} L\right] \\
& x \in W_{\operatorname{post}_{u}^{\mathcal{N}}(I), F} \Rightarrow \quad\left[\text { Since } W_{\operatorname{post}_{u}^{\mathcal{N}}(I), F}^{\mathcal{N}} \subseteq W_{\operatorname{post}_{v}^{\mathcal{N}}(I), F}^{\mathcal{N}}\right] \\
& x \in W_{\text {post }_{v}^{\mathcal{N}}(I), F} \Rightarrow \quad\left[\text { By definition of } W_{S, T}^{\mathcal{N}}\right] \\
& \exists q^{\prime} \in Q, x \in W_{q^{\prime}, F} \wedge v \in W_{I, q^{\prime}} \Rightarrow \quad\left[\text { Since } x \in W_{q^{\prime}, F} \Rightarrow W_{I, q^{\prime}} \subseteq L x^{-1}\right] \\
& v \in L x^{-1} \Rightarrow \quad\left[\text { Since } L x^{-1}=W_{I, q}\right] \\
& v \in W_{I, q} \Rightarrow \quad\left[\text { By definition of } \operatorname{post}_{v}^{\mathcal{N}}(I)\right] \\
& q \in \operatorname{post}_{v}^{\mathcal{N}}(I) \text {. }
\end{aligned}
$$


Therefore, $W_{\text {post }_{u}^{\mathcal{N}}(I), F}^{\mathcal{N}} \subseteq W_{\text {post }_{v}^{\mathcal{N}}(I), F}^{\mathcal{N}} \Rightarrow \operatorname{post}_{u}^{\mathcal{N}}(I) \subseteq \operatorname{post}_{v}^{\mathcal{N}}(I)$.

The proof for RFAs and left quasiorders is symmetric.

Finally, the following lemma shows that, for the Nerode's quasiorders, the $L$-composite principals can be described as intersections of $L$-prime principals.

LEMмA 6.2.4. Let $\mathcal{N}=\langle Q, \Sigma, \delta, I, F\rangle$ be an NFA with $\mathcal{L}(\mathcal{N})=L$. Then,

$$
u^{-1} L=\bigcup_{x \in \Sigma^{*}, x<{ }_{L}^{r} u} x^{-1} L \Longrightarrow \rho_{\leqslant_{L}^{r}}(u)=\bigcap_{x \in \Sigma^{*}, x<{ }_{L}^{r} u} \rho_{\leqslant_{L}^{r}}(x) \text {. }
$$

Similarly holds for the left Nerode's quasiorder $\leqslant_{L}^{\ell}$.

Proof. Observe that the inclusion $\rho_{\leqslant_{L}^{r}}(u) \subseteq \bigcap_{x \in \Sigma^{*}, x<{ }_{L}^{r} u} \rho_{\leqslant_{L}^{r}}(x)$ always holds since $x<{ }_{L}^{r} u \Rightarrow \rho_{\leqslant_{L}^{r}}(u) \subseteq \rho_{\leqslant_{L}^{r}}(x)$. Next, we prove the reverse inclusion.

Let $w \in \bigcap_{x \in \Sigma^{*}, x<{ }_{L}^{r} u} \rho_{\leqslant_{L}^{r}}(x)$ and assume that the left hand side of Equation (6.8) holds. Then, by definition of intersection and $\rho_{\leqslant_{L}^{r}}^{r}$, we have that $x \leqslant_{L}^{r} w$ for every $x \in \Sigma^{*}$ such that $x<_{L}^{r} u$, i.e., $x^{-1} L \subseteq w^{-1} L$ for every $x \in \Sigma^{*}$ such that $x^{-1} L \subsetneq u^{-1} L$. Since, by hypothesis, $u^{-1} L=\bigcup_{x \in \Sigma^{*}, x<_{L}^{r} u} x^{-1} L$, it follows that $u^{-1} L \subseteq w^{-1} L$ and, therefore, $w \in \rho_{\leqslant r}(u)$.

We conclude that $\bigcap_{x \in \Sigma^{*}, x<{ }_{L}^{r} u} \rho_{\leqslant_{L}^{r}}(x) \subseteq \rho_{\leqslant_{L}^{r}}(u)$.

\subsubsection{Automata Constructions}

In what follows, we will use Can and Res to denote the construction $\mathrm{H}$ when applied, respectively, to the language-based quasiorders induced by a regular language and the automata-based quasiorders induced by an NFA.

Definition 6.2.5 (Res and Can). Let $\mathcal{N}$ be an NFA with $L=\mathcal{L}(\mathcal{N})$. Define:

$$
\begin{array}{ll}
\operatorname{Can}^{r}(L) \stackrel{\text { def }}{=} \mathrm{H}^{r}\left(\leqslant_{L}^{r}, L\right) & \operatorname{Res}^{r}(\mathcal{N}) \stackrel{\text { def }}{=} \mathrm{H}^{r}\left(\leqslant_{\mathcal{N}}^{r}, L\right) \\
\operatorname{Can}^{\ell}(L) \stackrel{\text { def }}{=} \mathrm{H}^{\ell}\left(\leqslant_{L}^{\ell}, L\right) & \operatorname{Res}^{\ell}(\mathcal{N}) \stackrel{\text { def }}{=} \mathrm{H}^{\ell}\left(\leqslant_{\mathcal{N}}^{\ell}, L\right) .
\end{array}
$$

Given an NFA $\mathcal{N}$ generating the language $L=\mathcal{L}(\mathcal{N})$, all constructions in the above definition yield automata generating $L$. However, while the constructions using the right quasiorders result in RFAs, those using left quasiorders result in co-RFAs. Furthermore, it follows from Remark 6.2.1 and Lemma 6.1.7 that $\operatorname{Can}^{\ell}(L)$ is isomorphic to $\left(\operatorname{Can}^{r}\left(L^{R}\right)\right)^{R}$ and $\operatorname{Res}^{\ell}(\mathcal{N})$ is isomorphic to $\left(\operatorname{Res}^{r}\left(\mathcal{N}^{R}\right)\right)^{R}$.

It follows from Theorem 6.1.9 that the automata $\operatorname{Res}^{r}(\mathcal{N})$ and $\operatorname{Res}^{\ell}(\mathcal{N})$ have, at least, as many states as $\operatorname{Can}^{r}(L)$ and $\operatorname{Can}^{\ell}(L)$, respectively. Intuitively, $\operatorname{Can}^{r}(L)$ 
is the minimal RFA for $L$, i.e. it is isomorphic to the canonical RFA for $L$, since, as shown by de Luca and Varricchio [1994], $\leqslant_{L}^{r}$ is the coarsest right $L$-consistent quasiorder. On the other hand, as we shall see in Example 6.2.9, $\operatorname{Res}^{r}(\mathcal{N})$ is a sub-automaton of $\mathcal{N}^{\text {res }}$ [Denis et al. 2002] for every NFA $\mathcal{N}$.

Finally, it follows from Lemma 6.2.3 that residualizing $\left(\operatorname{Res}^{r}\right)$ a co-RFA (for instance, $\left.\operatorname{Res}^{\ell}(\mathcal{N})\right)$ results in the canonical RFA for $\mathcal{L}(\mathcal{N})\left(\operatorname{Can}^{r}(\mathcal{L}(\mathcal{N}))\right)$.

We formalize all these notions in Theorem 6.2.6.

THEOREM 6.2.6. Let $\mathcal{N}$ be an $N F A$ with $L=\mathcal{L}(\mathcal{N})$. Then the following hold:

(a) $\mathcal{L}\left(\operatorname{Can}^{r}(L)\right)=\mathcal{L}\left(\operatorname{Can}^{\ell}(L)\right)=L=\mathcal{L}\left(\operatorname{Res}^{r}(\mathcal{N})\right)=\mathcal{L}\left(\operatorname{Res}^{\ell}(\mathcal{N})\right)$.

(b) $\operatorname{Can}^{\ell}(L)$ is isomorphic to $\left(\operatorname{Can}^{r}\left(L^{R}\right)\right)^{R}$.

(c) $\operatorname{Res}^{\ell}(\mathcal{N})$ is isomorphic to $\left(\operatorname{Res}^{r}\left(\mathcal{N}^{R}\right)\right)^{R}$.

(d) $\operatorname{Can}^{r}(L)$ is isomorphic to the canonical RFA for $L$.

(e) $\operatorname{Res}^{r}(\mathcal{N})$ is isomorphic to a sub-automaton of $\mathcal{N}^{\text {res }}$.

(f) $\operatorname{Res}^{r}\left(\operatorname{Res}^{\ell}(\mathcal{N})\right)$ is isomorphic to $\operatorname{Can}^{r}(L)$.

Proof. In the following, let $\mathcal{N}=\langle Q, \Sigma, \delta, I, F\rangle$.

(a) By Definition 6.2.5, $\operatorname{Can}^{r}(L)=\mathrm{H}^{r}\left(\leqslant_{L}^{r}, L\right)$ and $\operatorname{Res}^{r}(\mathcal{N})=\mathrm{H}^{r}\left(\leqslant_{\mathcal{N}}^{r}, L\right)$. On the other hand, by Lemma 6.1.4, $\mathcal{L}\left(\mathrm{H}^{r}\left(\leqslant_{L}^{r}, L\right)\right)=\mathcal{L}\left(\mathrm{H}^{r}\left(\leqslant_{N}^{r}, L\right)\right)=L$. Therefore, $\mathcal{L}\left(\operatorname{Can}^{r}(L)\right)=\mathcal{L}\left(\operatorname{Res}^{r}(L)\right)=L$.

Similarly, it follows from Lemma 6.1.6 that $\mathcal{L}\left(\operatorname{Can}^{\ell}(L)\right)=\mathcal{L}\left(\operatorname{Res}^{\ell}(L)\right)=L$.

(b) For each $u, v \in \Sigma^{*}$ :

$$
\begin{array}{rlrl}
u \leqslant_{L}^{\ell} v \Leftrightarrow & & {[\text { By Definition (6.5)] }} \\
u^{-1} L \subseteq v^{-1} L \Leftrightarrow & & {\left[A \subseteq B \Leftrightarrow A^{R} \subseteq B^{R}\right]} \\
\left(u^{-1} L\right)^{R} \subseteq\left(v^{-1} L\right)^{R} \Leftrightarrow & & {\left[\text { Since }\left(u^{-1} L\right)^{R}=L^{R}\left(u^{R}\right)^{-1}\right]} \\
L^{R}\left(u^{R}\right)^{-1} \subseteq L^{R}\left(v^{R}\right)^{-1} & \Leftrightarrow & & {[\text { By Definition (6.4)] }} \\
u^{R} \leqslant_{L^{R}}^{r} v^{R} . & &
\end{array}
$$

Therefore, by Lemma 6.1.7, $\mathrm{Can}^{\ell}(L)$ is isomorphic to $\left(\operatorname{Can}^{r}\left(L^{R}\right)\right)^{R}$.

(c) For each $u, v \in \Sigma^{*}$ :

$$
\begin{aligned}
u \leqslant_{\mathcal{N}}^{\ell} v \Leftrightarrow & {[\text { By Definition (6.7)] }} \\
\operatorname{pre}_{u}^{\mathcal{N}^{R}}(F) \subseteq \operatorname{pre}_{v}^{\mathcal{N}^{R}}(F) \Leftrightarrow & {\left[\text { Since } q \in \operatorname{pre}_{x}^{\mathcal{N}^{R}}(F) \text { iff } q \in \operatorname{post}_{x^{R}}^{\mathcal{N}}(I)\right] } \\
\operatorname{post}_{u^{R}}^{\mathcal{N}}(I) \subseteq \operatorname{post}_{v^{R}}^{\mathcal{N}}(I) \Leftrightarrow & {[\text { By Definition (6.6)] }} \\
u^{R} \leqslant_{\mathcal{N}}^{\ell} v^{R} . &
\end{aligned}
$$


It follows from Lemma 6.1.7 that $\operatorname{Res}^{\ell}(\mathcal{N})$ is isomorphic to $\operatorname{Res}^{r}\left(\mathcal{N}^{R}\right)^{R}$.

(d) Let $\rho$ be the closure induced by $\leqslant_{L}^{r}$. Let $C=\langle\widetilde{Q}, \Sigma, \eta, \widetilde{I}, \widetilde{F}\rangle$ be the canonical RFA for $L$ and let $\operatorname{Can}^{r}(L)=\langle Q, \Sigma, \delta, I, F\rangle$. Let $\varphi: \widetilde{Q} \rightarrow Q$ be the mapping assigning to each state $\widetilde{q}_{i} \in \widetilde{Q}$ of the form $u^{-1} L$, the state $\rho(u) \in Q$, with $u \in \Sigma^{*}$.

We show that $\varphi$ is an NFA isomorphism between $C$ and $\operatorname{Can}^{r}(L)$.

Since

$$
u^{-1} L \subseteq L \Leftrightarrow u \leqslant_{L}^{r} \varepsilon \Leftrightarrow \varepsilon \in \rho(u),
$$

we have that $u^{-1} L$ is an initial state of $C$ iff $\rho(u)$ is an initial state of $\operatorname{Can}^{r}(L)$, hence $\varphi(\widetilde{I})=I$.

On the other hand, since

$$
\varepsilon \in u^{-1} L \Leftrightarrow u \in L,
$$

we have that $u^{-1} L$ is a final state of $C$ iff $\rho(u)$ is a final state of $\operatorname{Can}^{r}(L)$, hence $\varphi(\widetilde{F})=F$.

Moreover, since

$$
\rho(u) \cdot a \subseteq \rho(v) \Leftrightarrow v \leqslant_{L}^{r} u a \Leftrightarrow v^{-1} L \subseteq(u a)^{-1} L,
$$

we have that that $v^{-1} L=\eta\left(u^{-1} L, a\right)$ if and only if $\rho(v) \in \delta(\rho(u), a)$, for all $u^{-1} L, v^{-1} L \in \widetilde{q}$ and $a \in \Sigma$.

Finally, we need to show that $\forall u \in \Sigma^{*}, \rho(u) \in Q \Leftrightarrow \exists q_{i} \in \widetilde{Q}, q_{i}=u^{-1} L$. Observe that:

$$
\begin{aligned}
u^{-1} L=\bigcup_{x<L_{L}^{r} u} u^{-1} L \Leftrightarrow \quad \text { [By Definition (6.4)] } \\
u^{-1} L=\bigcup_{x^{-1} L \subset u^{-1} L} x^{-1} L .
\end{aligned}
$$

Therefore, $\forall u \in \Sigma^{*}, \rho(u)$ is $L$-prime $\Leftrightarrow u^{-1} L$ is prime, hence $\varphi(\widetilde{Q})=Q$.

(e) Recall that $\mathcal{N}^{\text {res }}=\left\langle Q_{r}, \Sigma, \delta_{r}, I_{r}, F_{r}\right\rangle$ is the RFA built by the residualization operation defined by Denis et al. [2002] (see Chapter 3). Let $\operatorname{Res}^{r}(\mathcal{N})=$ $\langle\widetilde{Q}, \Sigma, \widetilde{\delta}, \widetilde{I}, \widetilde{F}\rangle$.

Next, we show that there is a surjective mapping $\varphi$ that associates states and transitions of $\operatorname{Res}^{r}(\mathcal{N})$ with states and transitions of $\mathcal{N}^{\text {res }}$. Moreover, if $q \in \widetilde{Q}$ is initial (resp. final) then $\varphi(q) \in Q_{r}$ is initial (resp. final) and 
$q^{\prime} \in \widetilde{\delta}(q, a) \Leftrightarrow \varphi\left(q^{\prime}\right) \in \delta_{r}(\varphi(q), a)$. In this way, we conclude that $\operatorname{Res}^{r}(\mathcal{N})$ is isomorphic to a sub-automaton of $\mathcal{N}^{\text {res }}$.

Finally, since $\mathcal{L}\left(\mathcal{N}^{\text {res }}\right)=\mathcal{L}(\mathcal{N})$ then it follows from Lemma 6.1.4 that $\mathcal{L}\left(\mathcal{N}^{\text {res }}\right)=\mathcal{L}(\mathcal{N})=\mathcal{L}\left(\operatorname{Res}^{r}(\mathcal{N})\right)$.

Let $\rho$ be the closure induced by $\leqslant_{\mathcal{N}}^{r}$ and let $\varphi: \widetilde{Q} \rightarrow Q_{r}$ be the mapping assigning to each state $\rho(u) \in \widetilde{Q}$, the set $\operatorname{post}_{u}^{\mathcal{N}}(I) \in Q_{r}$ with $u \in \Sigma^{*}$.

Since

$$
\varepsilon \in \rho(u) \Leftrightarrow u \leqslant_{\mathcal{N}}^{r} \varepsilon \Leftrightarrow \operatorname{post}_{u}^{\mathcal{N}}(I) \subseteq \operatorname{post}_{\varepsilon}^{\mathcal{N}}(I)
$$

The initial states of $\operatorname{Res}^{r}(\mathcal{N})$ are mapped into the set the initial states of $\mathcal{N}^{\text {res }}$, hence $\varphi(\widetilde{I})=I_{r}$.

On the other hand, since

$$
\left.\rho(u) \subseteq L \Leftrightarrow u \in L \Leftrightarrow \operatorname{post}_{u}^{\mathcal{N}}(I) \cap F\right) \neq \varnothing,
$$

we have that the final states of $\operatorname{Res}^{r}(\mathcal{N})$, are mapped to the final states of $\mathcal{N}^{\text {res }}$, hence $\varphi(\widetilde{F})=F_{r}$.

Moreover, since

$$
\rho(u) \cdot a \subseteq \rho(v) \Leftrightarrow v \leqslant_{\mathcal{N}}^{r} u a \Leftrightarrow \operatorname{post}_{v}^{\mathcal{N}}(I) \subseteq \operatorname{post}_{u a}^{\mathcal{N}}(I),
$$

it follows that $\forall u, v \in \Sigma^{*}$ such that $\operatorname{post}_{u}^{\mathcal{N}}(I)$, $\operatorname{post}_{v}^{\mathcal{N}}(I) \in Q_{r}$, we have

$$
\operatorname{post}_{v}^{\mathcal{N}}(I) \in \delta_{r}\left(\operatorname{post}_{u}^{\mathcal{N}}(I), a\right) \Leftrightarrow \rho(v) \in \widetilde{\delta}(\rho(u), a) .
$$

Finally, we show that $\forall u \in \Sigma^{*}, \rho(u) \in \widetilde{Q} \Rightarrow \operatorname{post}_{u}^{\mathcal{N}}(I) \in Q_{r}$. By definition of $\widetilde{Q}$ and $Q_{r}$, this is equivalent to showing that for every word $u \in \Sigma^{*}$, if $\operatorname{post}_{u}^{\mathcal{N}}(I)$ is coverable then $\rho(u)$ is $L$-composite. Observe that:

$$
\begin{gathered}
\operatorname{post}_{u}^{\mathcal{N}}(I)=\bigcup_{\operatorname{post}_{x}^{\mathcal{N}}(I) \subset \operatorname{post}_{u}^{\mathcal{N}}(I)} \operatorname{post}_{x}^{\mathcal{N}}(I) \Leftrightarrow \quad\left[x<_{\mathcal{N}}^{r} u \Leftrightarrow \operatorname{post}_{x}^{\mathcal{N}}(I) \subset \operatorname{post}_{u}^{\mathcal{N}}(I)\right] \\
\operatorname{post}_{u}^{\mathcal{N}}(I)=\bigcup_{x<<_{\mathcal{N}}^{r}} \operatorname{post}_{x}^{\mathcal{N}}(I) \Rightarrow \quad\left[\operatorname{Since} W_{\operatorname{post}_{u}^{\mathcal{N}}(I), T}^{\mathcal{N}}=u^{-1} L\right] \\
u^{-1} L=\bigcup_{x<\mathcal{N}^{r} u} x^{-1} L .
\end{gathered}
$$

It follows that if $\operatorname{post}_{u}^{\mathcal{N}}(I)$ is coverable then $\rho(u)$ is $L$-composite, hence $\varphi(\widetilde{Q}) \subseteq Q_{r}$. 
(f) As shown by Lemma 6.1.6, $\operatorname{Res}^{\ell}(\mathcal{N})$ is a co-RFA with $\mathcal{L}\left(\operatorname{Res}^{\ell}(\mathcal{N})\right)=$ $\mathcal{L}(\mathcal{N})$. Therefore, it follows from Lemma 6.2.3 that $\operatorname{Res}^{r}\left(\operatorname{Res}^{\ell}(\mathcal{N})\right)$ is isomorphic to $\operatorname{Can}^{r}\left(\mathcal{L}\left(\operatorname{Res}^{\ell}(\mathcal{N})\right)\right)=\operatorname{Can}^{r}(\mathcal{L}(\mathcal{N}))$.

Figure 6.1 summarizes all the connections between the automata constructions given in Definition 6.2.5.

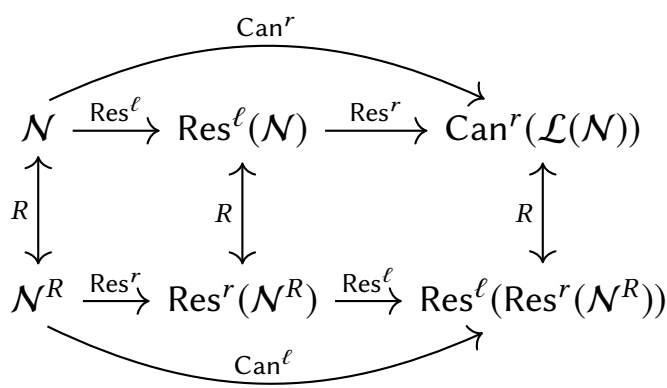

Figure 6.1: Relations between the constructions $\operatorname{Res}^{\ell}, \operatorname{Res}^{r}, \mathrm{Can}^{\ell}$ and $\operatorname{Can}^{r}$. Note that constructions $\mathrm{Can}^{r}$ and $\mathrm{Can}^{\ell}$ are applied to the language generated by the automaton in the origin of the labeled arrow while constructions $\operatorname{Res}^{r}$ and $\operatorname{Res}^{\ell}$ are applied directly to the automaton.

It is well-known that determinizing a deterministic automata yields the same automaton, i.e. $\mathcal{D}^{D}=\mathcal{D}$ for every DFA $\mathcal{D}$. As a consequence, determinizing twice and automaton is the same as doing it once, i.e. $\left(\mathcal{N}^{D}\right)^{D}=\mathcal{N}^{D}$. However, it is not clear that the same holds for our residualization operation, i.e. it is not clear whether $\operatorname{Res}^{r}\left(\operatorname{Res}^{r}(\mathcal{N})\right)=\operatorname{Res}^{r}(\mathcal{N})$.

The following lemma gives a sufficient condition on an RFA $\mathcal{H}$ built with our right automata construction so that applying our residualization operation results in the same automaton, i.e. $\operatorname{Res}^{r}(\mathcal{H})=\mathcal{H}$. In particular, we find that $\operatorname{Can}^{r}(L)$ is invariant to our residualization operation $\operatorname{Res}^{r}$.

LЕмма 6.2.7. Let $L$ be a regular language and let $\leqslant^{r}$ be a right L-consistent quasiorder. Let $\mathcal{H}=\mathrm{H}^{r}\left(\leqslant^{r}, L\right)$. If $\mathcal{H}$ is a strongly consistent RFA then $\leqslant_{\mathcal{H}}^{r}=\leqslant^{r}$.

Proof. Let $\mathcal{N}=\langle Q, \Sigma, \delta, I, F\rangle$ and $\mathcal{H}=\langle\widetilde{Q}, \Sigma, \widetilde{\delta}, \widetilde{I}, \widetilde{F}\rangle$. As shown by Lemma 6.1.4, $\mathcal{H}=\mathrm{H}^{r}\left(\leqslant^{r}, L\right)$ is an RFA generating $L$, hence each state of $\mathcal{H}$ is an $L$-prime principal $\rho_{\leqslant r}(u)$ whose right language is the quotient $u^{-1} L$ for some $u \in \Sigma^{*}$.

Observe that, by definition,

$$
\leqslant_{\mathcal{H}}^{r}=\leqslant^{r} \Leftrightarrow\left(\forall u, v \in \Sigma^{*}, \operatorname{post}_{u}^{\mathcal{H}}(\widetilde{I}) \subseteq \operatorname{post}_{v}^{\mathcal{H}}(\widetilde{I}) \Leftrightarrow u \leqslant^{r} v\right)
$$


Next we show that:

$$
\operatorname{post}_{u}^{\mathcal{H}}(\widetilde{I})=\left\{\rho_{\leqslant}(x) \in \widetilde{Q} \mid x \leqslant^{r} u\right\} .
$$

First, we prove that $\operatorname{post}_{u}^{\mathcal{H}}(\widetilde{I}) \subseteq\left\{\rho_{\leqslant}(x) \in \widetilde{Q} \mid x \leqslant^{r} u\right\}$. To simplify the notation, let $\rho$ denote $\rho_{\leqslant r}$.

$$
\begin{array}{rlrl}
\left.\rho(x) \in \operatorname{post}_{u}^{\mathcal{H}} \widetilde{I}\right) \Leftrightarrow & & \left.\left[\text { By definition of post }{ }_{u}^{\mathcal{H}} \widetilde{I}\right)\right] \\
\exists \rho\left(x_{0}\right) \in \widetilde{I}, u \in W_{\rho\left(x_{0}\right), \rho(x)}^{\mathcal{H}} & & {[\text { By Definition 6.1.3] }} \\
\exists \rho\left(x_{0}\right) \in \widetilde{Q}, \varepsilon \in \rho\left(x_{0}\right) \wedge \rho\left(x_{0}\right) \cdot u \subseteq \rho(x) \Leftrightarrow & & {[\text { By definition of } \rho]} \\
\exists \rho\left(x_{0}\right) \in \widetilde{Q}, x_{0} \leqslant^{r} \varepsilon \wedge x \leqslant^{r} u \cdot x_{0} & \Rightarrow & & {\left[\text { By mon. and trans. of } \leqslant^{r}\right]} \\
x \leqslant^{r} u . & &
\end{array}
$$

We now prove the reverse inclusion. Let $\rho(u), \rho(x) \in \widetilde{Q}$ be such that $x \leqslant^{r} u$. Then,

$$
\begin{aligned}
\rho(u) \in \widetilde{Q} & \Rightarrow[\text { By Lemma } 6.1 .4] \\
W_{\rho(u), F}^{\mathcal{H}}=u^{-1} L & \Rightarrow[\text { Since } \mathcal{H} \text { is str. cons. }] \\
u \in W_{I, \rho(u)}^{\mathcal{H}} & \Rightarrow\left[\text { By def. } W_{S, T}^{\mathcal{H}}, u=z a\right]
\end{aligned}
$$

$\exists \rho(y) \in \widetilde{Q}, \rho\left(u_{0}\right) \in \widetilde{I}, z \in W_{\rho\left(u_{0}\right), \rho(y)} \wedge a \in W_{\rho(y), \rho(u)} \Rightarrow$ [By Definition 6.1.3]

$$
\begin{aligned}
& z \in W_{\rho\left(u_{0}\right), \rho(y)} \wedge \rho(y) \cdot a \subseteq \rho(u) \Rightarrow \text { [By def. } \rho=\rho_{\leqslant r} \text { ] } \\
& z \in W_{\rho\left(u_{0}\right), \rho(y)} \wedge u \leqslant^{r} y \cdot a \Rightarrow \text { [Since } x \leqslant^{r} u \text { ] } \\
& z \in W_{\rho\left(u_{0}\right), \rho(y)} \wedge x \leqslant^{r} y a \Rightarrow \text { [By Def. 6.1.3] } \\
& z \in W_{\rho\left(u_{0}\right), \rho(y)} \wedge \rho(x) \in \delta(\rho(y), a) \Rightarrow\left[\text { By def. } \operatorname{post}_{u}(I)\right] \\
& \rho(x) \in \operatorname{post}_{u}(I) \text {. }
\end{aligned}
$$

It follows from Equation (6.9) that $\operatorname{post}_{u}^{\mathcal{H}}(I) \subseteq \operatorname{post}_{v}^{\mathcal{H}}(I) \Leftrightarrow u \leqslant^{r} v$, i.e. $\leqslant_{\mathcal{H}}^{r}=\leqslant^{r}$.

Finally, note that if $\leqslant_{L}^{r}=\leqslant_{\mathcal{N}}^{r}$ then clearly the automata $\operatorname{Can}^{r}(L)$ and $\operatorname{Res}^{r}(\mathcal{N})$ coincide for any NFA $\mathcal{N}$ with $L=\mathcal{L}(\mathcal{N})$. The following result shows that the reverse implication also holds.

LемMA 6.2.8. Let $\mathcal{N}$ be an NFA with $L=\mathcal{L}(\mathcal{N})$. Then $\leqslant_{L}^{r}=\leqslant_{\mathcal{N}}^{r}$ iff $\operatorname{Res}^{r}(\mathcal{N})$ is isomorphic to $\operatorname{Can}^{r}(L)$. 
Proof. As shown by Theorem 6.2.6(d), $\operatorname{Can}^{r}(L)$ is the canonical RFA for $L$, hence it is strongly consistent and, by Lemma 6.2.7, we have that $\leqslant_{\operatorname{Can}^{r}(L)}^{r}=\leqslant_{L}^{r}$. On the other hand, if $\operatorname{Res}^{r}(\mathcal{N})$ is isomorphic to $\operatorname{Can}^{r}(L)$ we have that $\leqslant_{\operatorname{Res}^{r}(\mathcal{N})}^{r}=$ $\leqslant_{\operatorname{Can}^{r}(L)}^{r}$, and by Lemma 6.2.7, $\leqslant_{\operatorname{Res}^{r}(\mathcal{N})}^{r}=\leqslant_{\mathcal{N}^{\prime}}^{r}$. It follows that if $\operatorname{Res}^{r}(\mathcal{N})$ is isomorphic to $\operatorname{Can}^{r}(L)$ then $\leqslant_{L}^{r}=\leqslant_{\mathcal{N}^{r}}^{r}$.

Finally, if $\leqslant_{L}^{r}=\leqslant_{\mathcal{N}}^{r}$ then $\mathrm{H}^{r}\left(\leqslant_{L}^{r}, L\right)=\mathrm{H}^{r}\left(\leqslant_{\mathcal{N}}^{r}, \mathcal{L}(\mathcal{N})\right)$, in other words, $\operatorname{Can}^{r}(L)=\operatorname{Res}^{r}(\mathcal{N})$.

The following example illustrates the differences between our residualization operation, $\operatorname{Res}^{r}(\mathcal{N})$, and the one defined by Denis et al. [2001], $\mathcal{N}^{\text {res }}$, on a given NFA $\mathcal{N}$ : the automaton $\operatorname{Res}^{r}(\mathcal{N})$ has, at most, as many states as $\mathcal{N}^{\text {res }}$. This follows from the fact that for every $u \in \Sigma^{*}$, if $\operatorname{post}_{u}^{\mathcal{N}}(I)$ is coverable then $\rho_{\leqslant_{\mathcal{N}}^{r}}(u)$ is composite but not vice-versa.

Example 6.2.9. Let $\mathcal{N}=\langle Q, \Sigma, \delta, I, F\rangle$ be the automata on the left of Figure 6.2 and let $L=\mathcal{L}(\mathcal{N})$. In order to build $\mathcal{N}^{\text {res }}$ we compute $\operatorname{post}_{u}^{\mathcal{N}}(I)$, for all $u \in \Sigma^{*}$. Let $C \stackrel{\text { def }}{=} L^{c} \backslash\{\varepsilon, a, b, c\}$.

$$
\begin{array}{lll}
\operatorname{post}_{\varepsilon}^{\mathcal{N}}(I)=\{0\} & \operatorname{post}_{a}^{\mathcal{N}}(I)=\{1,2\} & \forall w \in L, \operatorname{post}_{w}^{\mathcal{N}}(I)=\{5\} \\
\operatorname{post}_{c}^{\mathcal{N}}(I)=\{1,2,3,4\} & \operatorname{post}_{b}^{\mathcal{N}}(I)=\{1,3\} & \forall w \in C, \operatorname{post}_{w}^{\mathcal{N}}(I)=\varnothing
\end{array}
$$

Since none of these sets is coverable by the others, the are all states of $\mathcal{N}^{\text {res }}$. The resulting $R F A \mathcal{N}^{\text {res }}$ is shown in the center of Figure 6.2.

On the other hand, let us denote $\rho_{\leqslant_{N}^{r}}$ simply by $\rho$. In order to build $\operatorname{Res}^{r}(\mathcal{N})$ we need to compute the principals $\rho(u)$, for all $u \in \Sigma^{*}$. By definition of $\leqslant_{\mathcal{N}}^{r}$, we have that $w \in \rho(u) \Leftrightarrow \operatorname{post}_{u}^{\mathcal{N}}(I) \subseteq \operatorname{post}_{w}^{\mathcal{N}}(I)$. Therefore, we obtain:

$$
\begin{array}{lll}
\rho(\varepsilon)=\{\varepsilon\} & \rho(a)=\{a, c\} & \forall w \in L, \rho(w)=L \\
\rho(c)=\{c\} & \rho(b)=\{b, c\} & \forall w \in C, \rho(w)=\Sigma^{*}
\end{array}
$$

Since $a<_{\mathcal{N}}^{r} c, b<_{\mathcal{N}}^{r} c$ and $\forall w \in \Sigma^{*}, c w \subseteq L \Leftrightarrow(a w \subseteq L \vee b w \subseteq L)$, it follows that $\rho(c)$ is L-composite. The resulting $R F A \operatorname{Res}^{r}(\mathcal{N})$ is shown on the right of Figure 6.2.

\subsection{Double-Reversal Method for Building the Canonical RFA}

Denis et al. [2002] show that their residualization operation satisfies the residualequivalent of the double-reversal method for building the minimal DFA. More 


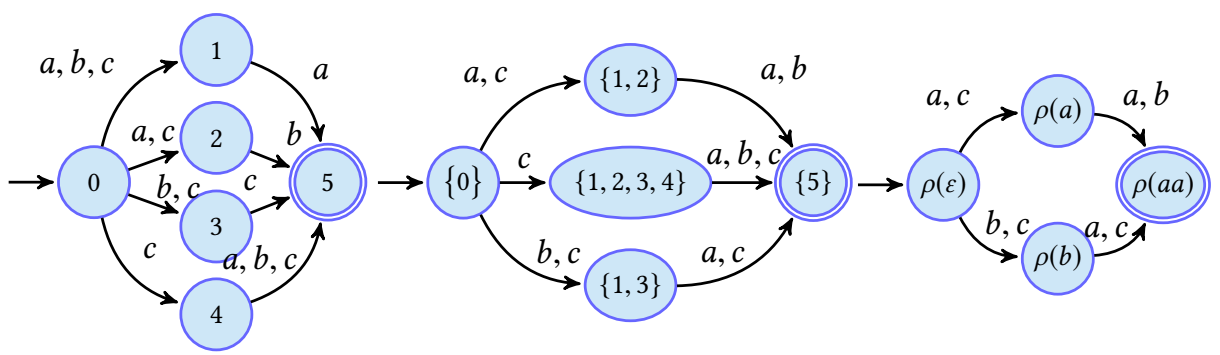

Figure 6.2: Form left to right: an NFA $\mathcal{N}$ and the $R F A s \mathcal{N}^{\text {res }}$ and $\operatorname{Res}^{r}(\mathcal{N})$. We omit the empty states for clarity.

specifically, they prove that if an NFA $\mathcal{N}$ is a co-RFA, then their residualization operation applied to $\mathcal{N}$ results in the canonical RFA for $\mathcal{L}(\mathcal{N})$. As a consequence,

$$
\left(\left(\left(\mathcal{N}^{R}\right)^{\text {res }}\right)^{R}\right)^{\text {res }} \text { is the canonical RFA for } \mathcal{L}(\mathcal{N}) .
$$

In this section we show that the residual-equivalent of the double-reversal method works when using our automata constructions based on quasiorders, i.e.

$$
\operatorname{Res}^{r}\left(\left(\operatorname{Res}^{r}\left(\mathcal{N}^{R}\right)\right)^{R}\right) \text { is isomorphic to } \operatorname{Can}^{r}(\mathcal{N}) \text {. }
$$

Then, we generalize this method along the lines of the generalization of the double-reversal method for building the minimal DFA given by Brzozowski and Tamm [2014].

To this end, we extend the work of Ganty et al. [2019] where they use congruences to offer a new perspective on the generalization of Brzozowski and Tamm [2014]. By switching from congruences to monotone quasiorders, we are able to give a necessary and sufficient condition on an NFA $\mathcal{N}$ that guarantees that our residualization operation yields the canonical RFA for $\mathcal{L}(\mathcal{N})$. Finally, we compare our generalization with the one given by Tamm [2015].

\subsubsection{Double-reversal Method}

We give a simple proof of the double-reversal method for building the canonical RFA for the language generated by a given NFA $\mathcal{N}$.

Theorem 6.3.1 (Double-Reversal). Let $\mathcal{N}$ be an NFA. Then $\operatorname{Res}^{r}\left(\left(\operatorname{Res}^{r}\left(\mathcal{N}^{R}\right)\right)^{R}\right)$ is isomorphic to the canonical RFA for $\mathcal{L}(\mathcal{N})$.

Proof. It follows from Theorem 6.2.6 (c), (d) and (f).

Note that Theorem 6.3.1 can be inferred from Figure 6.1 by following the path starting at $\mathcal{N}$, labeled with $R-\operatorname{Res}^{r}-R-\operatorname{Res}^{r}$ and ending in $\operatorname{Can}^{r}(\mathcal{L}(\mathcal{N}))$. 


\subsubsection{Generalization of the Double-reversal Method}

Next we show that residualizing an automaton yields the canonical RFA iff the left language of every state is closed w.r.t. the right Nerode quasiorder.

THEOREM 6.3.2. Let $\mathcal{N}=\langle Q, \Sigma, \delta, I, F\rangle$ be an NFA with $L=\mathcal{L}(\mathcal{N})$. Then $\operatorname{Res}^{r}(\mathcal{N})$ is the canonical RFA for $L$ iff $\forall q \in Q, \rho_{\leqslant_{L}^{r}}\left(W_{I, q}^{\mathcal{N}}\right)=W_{I, q}^{\mathcal{N}}$.

Proof. We first show that $\forall q \in Q, \rho_{\leqslant_{L}^{r}}\left(W_{I, q}^{\mathcal{N}}\right)=W_{I, q}^{\mathcal{N}}$ is a necessary condition, i.e. if $\operatorname{Res}^{r}(\mathcal{N})$ is the canonical RFA for $L$ then $\forall q \in Q, \rho_{\leqslant_{L}^{r}}\left(W_{I, q}^{\mathcal{N}}\right)=W_{I, q}^{\mathcal{N}}$ holds.

By Lemma 6.2.8 we have that if $\operatorname{Res}^{r}(\mathcal{N})$ is the canonical RFA for $L$ then $\leqslant_{L}^{r}=\leqslant_{\mathcal{N}}^{r}$. Moreover:

$$
\begin{aligned}
& \rho_{\leqslant_{L}^{r}}^{r}\left(W_{I, q}^{\mathcal{N}}\right)=\left[\text { By definition of } \rho_{\leqslant_{L}^{r}}\right] \\
&\left\{w \in \Sigma^{*} \mid \exists u \in W_{I, q}^{\mathcal{N}}, u^{-1} L \subseteq w^{-1} L\right\}=\left[\text { Since } \leqslant_{L}^{r}=\leqslant_{\mathcal{N}}^{r}\right] \\
&\left\{w \in \Sigma^{*} \mid \exists u \in W_{I, q}^{\mathcal{N}}, \operatorname{post}_{u}^{\mathcal{N}}(I) \subseteq \operatorname{post}_{w}^{\mathcal{N}}(I)\right\} \subseteq\left[\text { Since } u \in W_{I, q}^{\mathcal{N}} \Leftrightarrow q \in \operatorname{post}_{u}^{\mathcal{N}}(I)\right] \\
&\left\{w \in \Sigma^{*} \mid q \in \operatorname{post}_{w}^{\mathcal{N}}(I)\right\}=\left[\text { By definition of } W_{I, q}^{\mathcal{N}}\right] \\
& W_{I, q}^{\mathcal{N}} .
\end{aligned}
$$

By reflexivity of $\leqslant_{L}^{r}$, we conclude that $\rho_{\leqslant_{L}^{r}}\left(W_{I, q}^{\mathcal{N}}\right)=W_{I, q}^{\mathcal{N}}$.

Next, we show that $\forall q \in Q, \rho_{\leqslant_{L}^{r}}\left(W_{I, q}^{\mathcal{N}}\right)=W_{I, q}^{\mathcal{N}}$ is also a sufficient condition. By Lemma 6.2.2 and condition $\forall q \in Q, \rho_{\leqslant_{L}^{r}}\left(W_{I, q}^{\mathcal{N}}\right)=W_{I, q}^{\mathcal{N}}$, we have that

$$
\rho_{\leqslant_{\mathcal{N}}^{r}}(u)=\bigcap_{q \in \operatorname{post}_{u}^{\mathcal{N}}(I)} W_{I, q}^{\mathcal{N}}=\bigcap_{q \in \operatorname{post}_{u}^{\mathcal{N}}(I)} \rho_{\leqslant_{L}^{r}}\left(W_{I, q}^{\mathcal{N}}\right) .
$$

Since $u \in \rho_{\leqslant_{L}^{r}}\left(W_{I, q}^{\mathcal{N}}\right)$ for all $q \in \operatorname{post}_{u}^{\mathcal{N}}(I)$, it follows that $\rho_{\leqslant_{L}^{r}}(u) \subseteq \rho_{\leqslant_{L}^{r}}\left(W_{I, q}^{\mathcal{N}}\right)$ for all $q \in \operatorname{post}_{u}^{\mathcal{N}}(I)$ and, since $\rho_{\leqslant_{N}^{r}}(u)=\bigcap_{q \in \operatorname{post}_{u}^{\mathcal{N}}(I)} \rho_{\leqslant_{L}^{r}}\left(W_{I, q}^{\mathcal{N}}\right)$, we have that $\rho_{\leqslant_{L}^{r}}(u) \subseteq \rho_{\leqslant_{N}^{r}}(u)$ for every $u \in \Sigma^{*}$, i.e., $\leqslant_{L}^{r} \subseteq \leqslant_{\mathcal{N}}^{r}$.

On the other hand, as shown by de Luca and Varricchio [1994], we have that $\leqslant_{\mathcal{N}}^{r} \subseteq \leqslant_{L}^{r}$. We conclude that $\leqslant_{\mathcal{N}}^{r}=\leqslant_{L}^{r}$, hence $\operatorname{Res}^{r}(\mathcal{N})=\operatorname{Can}^{r}(L)$.

It is worth to remark that Theorem 6.3.2 does not hold when considering the residualization operation $\mathcal{N}^{\text {res }}$ [Denis et al. 2002]. As a counterexample we have the automaton $\mathcal{N}$ in Figure 6.2 where $\operatorname{Res}^{r}(\mathcal{N})$ is the canonical RFA for $\mathcal{L}(\mathcal{N})$, hence $\mathcal{N}$ satisfies the condition of Theorem 6.3.2, while $\mathcal{N}^{\text {res }}$ is not canonical. 


\subsubsection{Co-atoms and co-rests}

The condition of Theorem 6.3.2 is analogue to the one Ganty et al. [2019, Theorem 16] give for building the minimal DFA, except that the later is formulated in terms of congruences instead of quasiorders. In that case they prove that, given an NFA $\mathcal{N}=\langle Q, \Sigma, \delta, I, F\rangle$ with $L=\mathcal{L}(\mathcal{N})$,

$\mathcal{N}^{D}$ is the minimal DFA for $L$ iff $\forall q \in Q, \rho_{\sim_{L}^{r}}\left(W_{I, q}^{\mathcal{N}}\right)=W_{I, q}^{\mathcal{N}}$,

where $\sim_{L}^{r} \stackrel{\text { def }}{=} \leqslant_{L}^{r} \cap\left(\leqslant_{L}^{r}\right)^{-1}$ is the right Nerode's congruence.

Moreover, Ganty et al. [2019] show that the principals of $\sim_{L}^{r}$ coincide with the so-called co-atoms [Ganty et al. 2019], which are non-empty intersections of complemented and uncomplemented right quotients of the language. This allowed them to connect their result with the generalization of the double-reversal method for DFAs of Brzozowski and Tamm [2014], who establish that determinizing an NFA $\mathcal{N}$ yields the minimal DFA for $\mathcal{L}(\mathcal{N})$ iff the left languages of the states of $\mathcal{N}$ are unions of co-atoms of $\mathcal{L}(\mathcal{N})$.

Next, we give a formulation of the condition from Theorem 6.3.2 along the lines of the one given by Brzozowski and Tamm [2014] for their generalization of the double-reversal method for building the minimal DFA.

To do that, let us call the intersections used in Lemma 6.2.2 to describe the principals of $\leqslant_{L}^{\ell}$ and $\leqslant_{L}^{r}$ as rests and co-rests of $L$, respectively.

Definition 6.3.3 (Rest and Co-rest). Let $L$ be a regular language. A rest (resp. co-rest) is any non-empty intersection of left (resp. right) quotients of $L$.

As shown by Theorem 6.3.2, residualizing an NFA $\mathcal{N}$ yields the canonical RFA for $\mathcal{L}(\mathcal{N})$ iff the left language of every state of $\mathcal{N}$ satisfies $\rho_{\leqslant_{L}^{r}}\left(W_{I, q}^{\mathcal{N}}\right)=W_{I, q}^{\mathcal{N}}$. By definition, $\rho_{\leqslant_{L}^{r}}(S)=S$ iff $S$ is a union of principals of $\leqslant_{L}^{r}$.

Therefore we derive the following statement, equivalent to Theorem 6.3.2, that we consider as the residual-equivalent of the generalization of the doublereversal for building the minimal DFA [Brzozowski and Tamm 2014].

COROLlarY 6.3.4. Let $\mathcal{N}$ be an NFA with $L=\mathcal{L}(\mathcal{N})$. Then $\operatorname{Res}^{r}(\mathcal{N})$ is the canonical RFA for $L$ iff the left languages of $\mathcal{N}$ are union of co-rests.

\subsubsection{Tamm's Generalization of the Double-reversal Method for RFAs}

Tamm [2015] generalized the double-reversal method of Denis et al. [2002] by showing that $\mathcal{N}^{\text {res }}$ is the canonical RFA for $\mathcal{L}(\mathcal{N})$ iff the left languages of $\mathcal{N}$ are union of the left languages of the canonical RFA for $\mathcal{L}(\mathcal{N})$. 
In this section, we compare the generalization of Tamm [2015] with ours. The two approaches differ in the definition of the residualization operation they consider and, as we show next, the sufficient and necessary condition from Theorem 6.3.2 is more general than that of Tamm [2015, Theorem 4].

Lемма 6.3.5. Let $\mathcal{N}=\langle Q, \Sigma, \delta, I, F\rangle$ be an $N F A$ with $L=\mathcal{L}(\mathcal{N})$ and let $C=\operatorname{Can}^{r}\left(\leqslant_{L}^{r}, L\right)=\langle\widetilde{Q}, \Sigma, \widetilde{\delta}, \widetilde{I}, \widetilde{F}\rangle$ be the canonical RFA for $L$. Then

$$
W_{I, q}^{\mathcal{N}}=\bigcup_{q \in \widetilde{Q}} W_{\widetilde{I}, q}^{C} \Longrightarrow \rho_{\leqslant_{L}^{r}}\left(W_{I, q}^{\mathcal{N}}\right)=W_{I, q}^{\mathcal{N}}
$$

Proof. Since the canonical RFA, $C$, is strongly consistent then it follows from Lemma 6.2.7 that $\leqslant_{C}^{r}=\leqslant_{L}^{r}$ and, consequently, $\operatorname{Res}^{r}(C)$ is isomorphic to $\operatorname{Can}^{r}(L)$. It follows from Theorem 6.3 .2 that $\rho_{\leqslant_{L}^{r}}\left(W_{\widetilde{I}, q}^{C}\right)=W_{\widetilde{I}, q}^{C}$ for every $q \in \widetilde{Q}$. Therefore,

$$
\begin{aligned}
\rho_{\leqslant_{L}^{r}}\left(W_{I, q}^{\mathcal{N}}\right)= & {\left[\text { Since } W_{I, q}^{\mathcal{N}}=\bigcup_{q \in \widetilde{Q}} W_{\widetilde{I}, q}^{C} \text { and } \rho_{\leqslant_{L}^{r}}\left(\cup S_{i}\right)=\cup \rho_{\leqslant_{L}^{r}}\left(S_{i}\right)\right] } \\
\cup_{q \in \widetilde{Q}} \rho_{\leqslant_{L}^{r}}\left(W_{\widetilde{I}, q}^{C}\right)= & {\left[\text { Since } \rho_{\leqslant_{L}^{r}}\left(W_{\widetilde{I}, q}^{C}\right)=W_{\widetilde{I}, q}^{C} \text { for every } q \in \widetilde{Q}\right] } \\
\bigcup_{q \in \widetilde{Q}} W_{\widetilde{I}, q}^{C} . &
\end{aligned}
$$

Observe that, since the canonical RFA $C=\langle\widetilde{Q}, \Sigma, \widetilde{\delta}, \widetilde{I}, \widetilde{F}\rangle$ for a language $L$ is strongly consistent, the left language of each state is a principal of $\leqslant_{L}^{r}$. In particular, if the right language of a state is $u^{-1} L$ then its left language is the principal $\rho_{\leqslant_{L}^{r}}(u)$. Therefore, if $W_{I, q}^{\mathcal{N}}=\cup_{q \in \widetilde{Q}} W_{\widetilde{I}, q}^{C}$ then $W_{I, q}^{\mathcal{N}}$ is a closed set in $\rho_{\leqslant_{L}^{r}}^{r}$. However, the reverse implication does not hold since only the L-prime principals are left languages of states of $C$.

On the other hand, Lemma 6.2.4 shows that $L$-composite principals can be described as intersections of $L$-prime principals when we consider the Nerode's quasiorder $\leqslant_{L}^{r}$. As a consequence, our residualization operation applied on an NFA $\mathcal{N}$ yields the canonical RFA for $\mathcal{L}(\mathcal{N})$ iff the left languages of states of $\mathcal{N}$ are union of non-empty intersections of left languages of the canonical RFA while Tamm [2015] proves that $\mathcal{N}^{\text {res }}$ yields to the canonical RFA iff the left languages of states of $\mathcal{N}$ are union of left languages of the canonical RFA.

\subsection{Learning Residual Automata}

Bollig et al. [2009] devised the $\mathrm{NL}^{*}$ algorithm for learning the canonical RFA for a given regular language. The algorithm describes the behavior of a Learner that infers a language $L$ by performing membership queries on $L$ (which are answered 
by a Teacher) and equivalence queries between the language generated by a candidate automaton and $L$ (which are answered by an Oracle). The algorithm terminates when the Learner builds an RFA generating the language $L$.

For the shake of completeness, we offer an overview of the $\mathrm{NL}^{*}$ algorithm as presented by Bollig et al. [2009].

\subsubsection{The NL* Algorithm [Bollig et al. 2009]}

The Learner maintains a prefix-closed finite set $\mathcal{P} \subseteq \Sigma^{*}$ and a suffix-closed finite set $\mathcal{S} \subseteq \Sigma^{*}$. The Learner groups the words in $\mathcal{P}$ by building a table $T=(\mathcal{T}, \mathcal{P}, \mathcal{S})$ where $T:(\mathcal{P} \cup \mathcal{P} \cdot \Sigma) \times \mathcal{S} \rightarrow\{+,-\}$ is a function such that for every $u \in \mathcal{P} \cup \mathcal{P} \cdot \Sigma$ and $v \in \mathcal{S}$ we have that $T(u, v)=+\Leftrightarrow u v \in L$. Otherwise $T(u, v)=-$.

For every word $u \in \mathcal{P} \cup \mathcal{P} \cdot \Sigma$, define the function $\mathrm{r}(u): \mathcal{S} \rightarrow\{+,-\}$ as $\mathrm{r}(u)(v) \stackrel{\text { def }}{=} T(u, v)$. The set of all rows of a table $\mathcal{T}$ is denoted by $\operatorname{Rows}(\mathcal{T})$.

The algorithm uses the table $\mathcal{T}=(T, \mathcal{P}, \mathcal{S})$ to build an automaton whose states are some of the rows of $\mathcal{T}$. In order to do that, it is necessary to define the notions of union of rows, prime row and composite row.

Definition 6.4.1 (Join Operator). Let $\mathcal{T}=(T, \mathcal{P}, \mathcal{S})$ be a table. For every pair of rows $r_{1}, r_{2} \in \operatorname{Rows}(\mathcal{T})$, define the join $r_{1} \sqcup r_{2}: \mathcal{S} \rightarrow\{+,-\}$ as:

$$
\forall x \in \mathcal{S},\left(r_{1} \sqcup r_{2}\right)(x) \stackrel{\text { def }}{=} \begin{cases}+ & \text { if } r_{1}(x)=+\vee r_{2}(x)=+ \\ - & \text { otherwise }\end{cases}
$$

Note that the join operator is associative, commutative and idempotent. However, the join of two rows is not necessarily a row of $\mathcal{T}$.

Definition 6.4.2 (Covering Relation). Let $\mathcal{T}=(T, \mathcal{P}, \mathcal{S})$ be a table. Then, for every pair of rows $r_{1}, r_{2} \in \operatorname{Rows}(\mathcal{T})$ we have that

$$
r_{1} \sqsubseteq r_{2} \stackrel{\text { def }}{\Leftrightarrow} \forall x \in \mathcal{S}, r_{1}(x)=+\Rightarrow r_{2}(x)=+.
$$

We write $r_{1} \sqsubset r_{2}$ to denote $r_{1} \sqsubseteq r_{2}$ and $r_{1} \neq r_{2}$.

Definition 6.4.3 (Composite and Prime Rows). Let $\mathcal{T}=(T, \mathcal{P}, \mathcal{S})$ be a table. We say a row $r \in \operatorname{Rows}(\mathcal{T})$ is $\mathcal{T}$-composite if it is the join of all the rows that it strictly covers, i.e. $r=\bigsqcup_{r^{\prime} \in \operatorname{Rows}}(\mathcal{T}), r^{\prime} \sqsubset r r^{\prime}$. Otherwise, we say $r$ is $\mathcal{T}$-prime.

Definition 6.4.4 (Closed and Consistent Table). Let $\mathcal{T}=(T, \mathcal{P}, \mathcal{S})$ be a table. Then

(a) $\mathcal{T}$ is closed iff

$$
\forall u \in \mathcal{P}, a \in \Sigma, \mathrm{r}(u a)=\bigsqcup\{\mathrm{r}(v) \mid v \in \mathcal{P}, \mathrm{r}(v) \sqsubseteq \mathrm{r}(u a) \wedge \mathrm{r}(v) \text { is } \mathcal{T} \text {-prime }\} .
$$


(b) $\mathcal{T}$ is consistent iff

$$
\mathrm{r}(u) \sqsubseteq \mathrm{r}(v) \Rightarrow \mathrm{r}(u a) \sqsubseteq \mathrm{r}(v a) \text { for every } u, v \in \mathcal{P} \text { and } a \in \Sigma .
$$

At each iteration of the algorithm, the Learner checks whether the current table $\mathcal{T}=(T, \mathcal{P}, \mathcal{S})$ is closed and consistent.

If $\mathcal{T}$ is not closed, then it finds $\mathrm{r}(u a)$ with $u \in \mathcal{P}, a \in \Sigma$ such that $\mathrm{r}(u a)$ is $\mathcal{T}$-prime and it is not equal to some $\mathrm{r}(v)$ with $v \in \mathcal{P}$. Then the Learner adds $u a$ to $\mathcal{P}$ and updates the table $\mathcal{T}$.

Similarly, if $\mathcal{T}$ is not consistent, the Learner finds $u, v \in \mathcal{P}, a \in \Sigma, x \in \mathcal{S}$ such that $\mathrm{r}(u) \subseteq \mathrm{r}(v)$ but $\mathrm{r}(u a)(x)=+\wedge \mathrm{r}(v a)(x)=-$. Then the Learner adds ax to $\mathcal{S}$ and updates $\mathcal{T}$.

When the table $\mathcal{T}$ is closed and consistent, the Learner builds the RFA $\operatorname{R}(\mathcal{T})$.

Definition 6.4.5 (Automata Construction $\mathrm{R}(\mathcal{T})$ ). Let $\mathcal{T}=(T, \mathcal{P}, \mathcal{S})$ be a closed and consistent table. Define the automaton $\mathrm{R}(\mathcal{T}) \stackrel{\text { def }}{=}\langle Q, \Sigma, \delta, I, F\rangle$ with $Q=\{\mathrm{r}(u) \mid$ $u \in \mathcal{P} \wedge \mathrm{r}(u)$ is $\mathcal{T}$-prime $\}, I=\{\mathrm{r}(u) \in Q \mid \mathrm{r}(u) \sqsubseteq \mathrm{r}(\varepsilon)\}, F=\{\mathrm{r}(u) \in Q \mid \mathrm{r}(u)(\varepsilon)=+\}$ and $\mathrm{r}(v) \in \delta(\mathrm{r}(u), a)=\{\mathrm{r}(v) \in Q \mid \mathrm{r}(v) \sqsubseteq \mathrm{r}(u a)\}$ for all $\mathrm{r}(u) \in Q, a \in \Sigma$.

The Learner asks the Oracle whether $\mathcal{L}(\mathrm{R}(\mathcal{T}))=L$. If the Oracle answers yes then the algorithm terminates. Otherwise, the Oracle returns a counterexample $w$ for the language equivalence. Then the Learner adds every suffix of $w$ to $\mathcal{S}$, updates the table $\mathcal{T}$ and repeats the process.

\subsubsection{The $\mathrm{NL}^{\leqslant}$Algorithm}

In this section we present a quasiorder-based perspective on the $\mathrm{NL}^{*}$ algorithm in which the Learner iteratively refines a quasiorder on $\Sigma^{*}$ by querying the Teacher and uses and adaption of the automata construction from Definition 6.1.3 to build an RFA that is used to query the Oracle. We capture this approach in the so-called $N L^{\leqslant}$algorithm.

Next we explain the behavior of algorithm $\mathrm{NL}^{\leqslant}$and give the necessary definitions in order to understand it and its relation with the algorithm $\mathrm{NL}^{*}$.

The Learner maintains a prefix-closed finite set $\mathcal{P} \subseteq \Sigma^{*}$ and a suffix-closed finite set $\mathcal{S} \subseteq \Sigma^{*}$. The set $\mathcal{S}$ is used to approximate the principals in $\leqslant_{L}^{r}$ for the words in $\mathcal{P}$. In order to manipulate these approximations, we define the following two operators.

Definition 6.4.6. Let $L$ be a language, $\mathcal{S} \subseteq \Sigma^{*}$ and $u \in \Sigma^{*}$. Define:

$$
\begin{aligned}
& u^{-1} L=\mathcal{S} v^{-1} L \stackrel{\text { def }}{\Leftrightarrow}\left(u^{-1} L \cap \mathcal{S}\right)=\left(v^{-1} L \cap \mathcal{S}\right) \\
& u^{-1} L \subseteq \mathcal{S} v^{-1} L \stackrel{\text { def }}{\Leftrightarrow}\left(u^{-1} L \cap \mathcal{S}\right) \subseteq\left(v^{-1} L \cap \mathcal{S}\right) .
\end{aligned}
$$




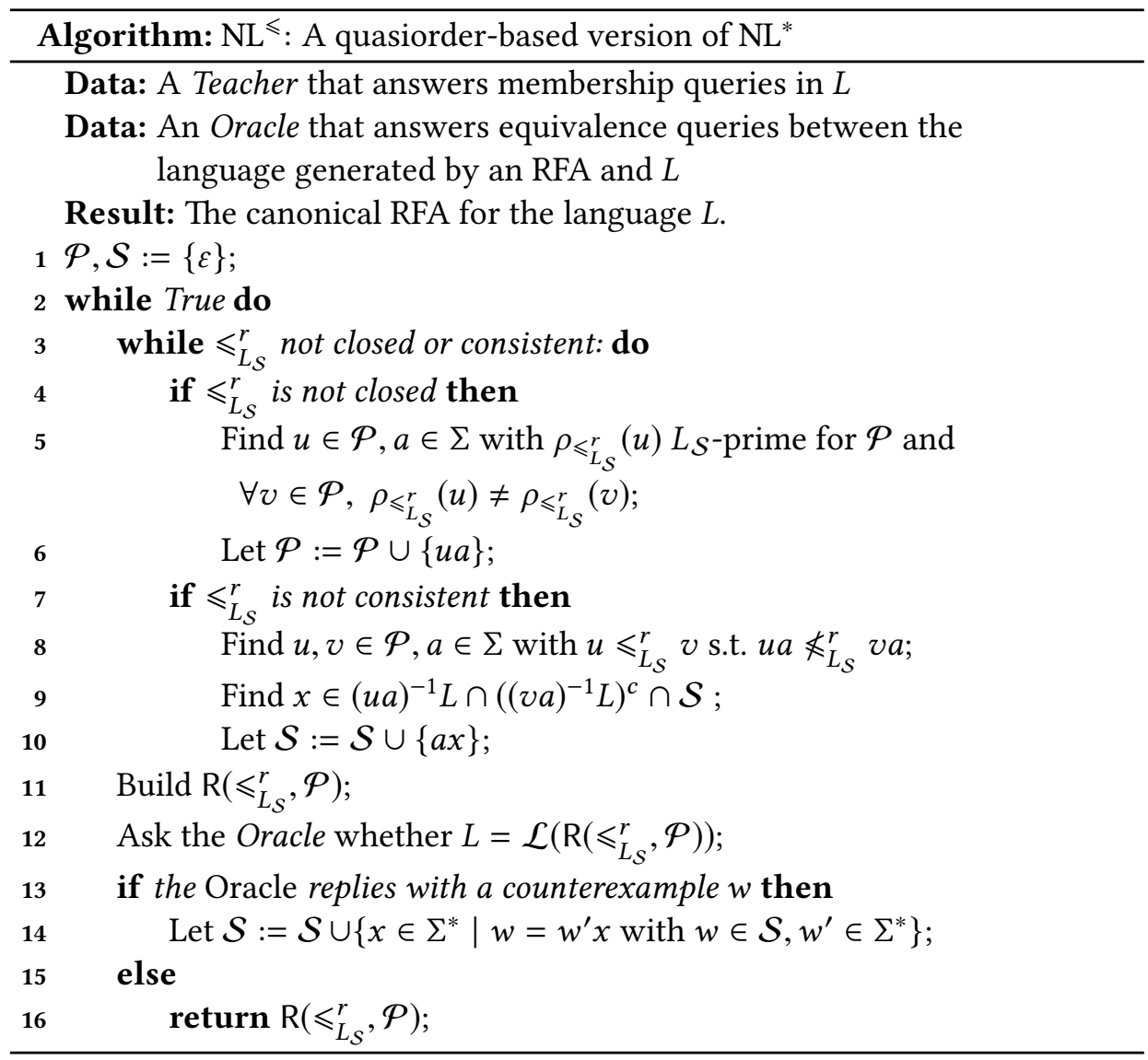

These operators allow us to define an over-approximation of Nerode's quasiorder that can be decided with finitely many membership tests.

Definition 6.4.7 (Right-language-based quasiorder w.r.t. $\mathcal{S}$ ). Let $L$ be a language, $\mathcal{S} \subseteq \Sigma^{*}$ and $u, v \in \Sigma^{*}$. Define $u \leqslant_{L_{\mathcal{S}}}^{r} v \stackrel{\text { def }}{\Leftrightarrow} u^{-1} L \subseteq \mathcal{S} v^{-1} L$.

Recall that the Learner only manipulates the principals for the words in $\mathcal{P}$. Therefore, we need to adapt the notion of composite principal for $\leqslant_{L_{\mathcal{S}}}^{r}$.

Definition 6.4.8 $\left(L_{\mathcal{S}}\right.$-Composite Principal w.r.t. $\left.\mathcal{P}\right)$. Let $\mathcal{P}, \mathcal{S} \subseteq \Sigma^{*}$ with $u \in \mathcal{P}$ and let $L \subseteq \Sigma^{*}$ be a language. We say $\rho_{\leqslant_{L_{\mathcal{S}}}^{r}}(u)$ is $L_{\mathcal{S}}$-composite w.r.t. $\mathcal{P}$ iff

$$
u^{-1} L=\mathcal{S} \bigcup_{x \in \mathcal{P}, x<L_{L_{\mathcal{S}}}^{r} u} x^{-1} L .
$$

Otherwise, we say it is $L_{\mathcal{S}}$-prime w.r.t. $\mathcal{P}$. 
The Learner uses the quasiorder $\leqslant_{L_{\mathcal{S}}}^{r}$ to build an automaton by adapting the construction from Definition 6.1.3 in order to use only the information that is available by means of the sets $\mathcal{S}$ and $\mathcal{P}$. Building such an automaton requires the quasiorder to satisfy two conditions: it must be closed and consistent w.r.t. $\mathcal{P}$.

Definition 6.4.9 (Closedness and Consistency of $\leqslant_{L_{\mathcal{S}}}^{r}$ w.r.t. $\mathcal{P}$ ).

(a) $\leqslant_{L_{\mathcal{S}}}^{r}$ is closed w.r.t. $\mathcal{P}$ iff

$\forall u \in \mathcal{P}, a \in \Sigma, \rho_{L_{\mathcal{S}}^{r}}(u a)$ is $L_{\mathcal{S}}$-prime w.r.t. $\mathcal{P} \Rightarrow \exists v \in \mathcal{P}, \rho_{\leqslant_{L_{\mathcal{S}}^{r}}^{r}}(u a)=\rho_{\leqslant_{L_{\mathcal{S}}}^{r}}(v)$.

(b) $\leqslant_{L_{\mathcal{S}}}^{r}$ is consistent w.r.t. $\mathcal{P}$ iff

$$
\forall u, v \in \mathcal{P}, a \in \Sigma: u \leqslant_{L_{\mathcal{S}}}^{r} v \Rightarrow u a \leqslant_{L_{\mathcal{S}}}^{r} v a .
$$

At each iteration, the Learner checks whether the quasiorder $\leqslant_{L_{\mathcal{S}}}^{r}$ is closed and consistent w.r.t. $\mathcal{P}$. If $\leqslant_{L_{\mathcal{S}}}^{r}$ is not closed w.r.t. $\mathcal{P}$, then it finds $\rho_{\leqslant_{L_{\mathcal{S}}}^{r}}(u a)$ with $u \in \mathcal{P}, a \in \sum$ such that $\rho_{\leqslant_{L_{\mathcal{S}}}^{r}}(u a)$ is $L_{\mathcal{S}}$-prime w.r.t. $\mathcal{P}$ and it is not equal to some $\rho_{L_{\mathcal{S}}^{r}}(v)$ with $v \in \mathcal{P}$. Then the Learner adds $u a$ to $\mathcal{P}$.

Similarly, if $\leqslant_{L_{\mathcal{S}}}^{r}$ is not consistent w.r.t. $\mathcal{P}$ then the Learner finds $u, v \in \mathcal{P}$, $a \in \Sigma, x \in \mathcal{S}$ such that $u \leqslant_{L_{\mathcal{S}}}^{r} v$ but $u a x \in L \wedge$ vax $\notin L$. Then the Learner adds ax to $\mathcal{S}$. When the quasiorder $\leqslant_{L_{\mathcal{S}}}^{r}$ is closed and consistent w.r.t. $\mathcal{P}$, the Learner builds the RFA $\mathrm{R}\left(\leqslant_{L_{\mathcal{S}}}^{r}, \mathcal{P}\right)$.

Definition 6.4.10 is an adaptation of the automata construction $\mathrm{H}^{r}$ from Definition 6.1.3. Instead of considering all principals, it considers only those that correspond to words in $\mathcal{P}$. Moreover, the notion of $L$-primality is replaced by $L_{\mathcal{S}}-$ primality w.r.t. $\mathcal{P}$ since the algorithm does not manipulate quotients of $L$ by words in $\Sigma^{*}$ but the approximation through $\mathcal{S}$ of the quotients of $L$ by words in $\mathcal{P}$ (see Definition 6.4.6).

Definition 6.4.10 (Automata construction $\mathrm{L}\left(\leqslant_{L_{\mathcal{S}}}^{r}, \mathcal{P}\right)$ ). Let $L \subseteq \Sigma^{*}$ be a regular language and let $\mathcal{P}, \mathcal{S} \subseteq \Sigma^{*}$. Define the automaton $\mathrm{L}\left(\leqslant_{L_{\mathcal{S}}}^{r}, \mathcal{P}\right)=\langle Q, \Sigma, \delta, I, F\rangle$ with $Q=\left\{\rho_{\leqslant_{L_{\mathcal{S}}}^{r}}(u) \mid u \in \mathcal{P}, \rho_{\leqslant_{L_{\mathcal{S}}}^{r}}(u)\right.$ is $L_{\mathcal{S}}$-prime w.r.t. $\left.\mathcal{P}\right\}, I=\left\{\rho_{\leqslant_{L_{\mathcal{S}}}^{r}}(u) \in Q \mid\right.$ $\left.\varepsilon \in \rho_{\leqslant_{L_{\mathcal{S}}}^{r}}(u)\right\}, F=\left\{\rho_{\leqslant_{L_{\mathcal{S}}}^{r}}(u) \in Q \mid u \in L\right\}$ and $\delta\left(\rho_{\leqslant_{L_{\mathcal{S}}}^{r}}(u), a\right)=\left\{\rho_{L_{L_{\mathcal{S}}}^{r}}(v) \in Q \mid\right.$ $\left.\rho_{\Sigma_{L_{\mathcal{S}}}^{r}}(u) a \subseteq \rho_{\leftarrow_{L_{\mathcal{S}}}^{r}}(v)\right\}$ for all $\rho_{\leqslant_{L_{\mathcal{S}}}^{r}}(u) \in Q$ and $a \in \Sigma$.

Finally, the Learner asks the Oracle whether $\mathcal{L}\left(\mathrm{R}\left(\leqslant_{L_{\mathcal{S}}}^{r}, \mathcal{P}\right)\right)=L$. If the Oracle answers yes then the algorithm terminates. Otherwise, the Oracle returns a counterexample $w$ for the language equivalence. Then, the Learner adds every suffix of $w$ to $\mathcal{S}$ and repeats the process.

Theorem 6.4.11 shows that the $\mathrm{NL}^{\leqslant}$algorithm exactly coincides with $\mathrm{NL}^{*}$. 
THEOREM 6.4.11. $N L^{\leqslant}$builds the same sets $\mathcal{P}$ and $\mathcal{S}$, performs the same queries to the Oracle and the Teacher and returns the same RFA as $N L^{*}$, provided that both algorithms perform the same non-deterministic choices.

Proof. Let $\mathcal{P}, \mathcal{S} \subseteq \Sigma^{*}$ be a prefix-closed and a suffix-closed finite set, respectively, and let $\mathcal{T}=(T, \mathcal{P}, \mathcal{S})$ be the table built by algorithm $\mathrm{NL}^{*}$. Observe that for every $u, v \in \mathcal{P}$ :

$$
\begin{aligned}
u \leqslant_{L_{\mathcal{S}}}^{r} v \Leftrightarrow & & \text { [By Definition 6.4.7] } \\
u^{-1} L \subseteq \mathcal{S} v^{-1} L \Leftrightarrow & & \text { [By definition of quotient w.r.t } S \text { ] } \\
\forall x \in S, u x \in L \Rightarrow v x \in L \Leftrightarrow & & \text { [By definition of } \mathcal{T} \text { ] } \\
\forall x \in S,(\mathrm{r}(u)(x)=+) \Rightarrow(\mathrm{r}(v)(x)=+) \Leftrightarrow & & \text { [By Definition 6.4.2] } \\
\mathrm{r}(u) \sqsubseteq \mathrm{r}(v) . & &
\end{aligned}
$$

Moreover, for every $u, v \in \mathcal{P}$ we have that $u^{-1} L=\mathcal{S} v^{-1} L$ iff $\mathrm{r}(u)=\mathrm{r}(v)$.

Next, we show that the join operator applied to rows corresponds to the set union applied to quotients w.r.t $S$. Let $u, v \in \mathcal{P}$ and let $x \in \mathcal{S}$. Then,

$$
\begin{array}{rll}
(\mathrm{r}(u) \sqcup \mathrm{r}(v))(x)=+\Leftrightarrow & {[\text { By Definition 6.4.1] }} \\
(\mathrm{r}(u)(x)=+) \vee(\mathrm{r}(v)(x)=+) \Leftrightarrow & {[\text { By definition of row }]} \\
(u x \in L) \vee(v x \in L) \Leftrightarrow & {[\text { By definition of quotient w.r.t } \mathcal{S} \text { ] }} \\
\left(x \in u^{-1} L\right) \vee\left(x \in v^{-1} L\right) \Leftrightarrow & {[\text { By definition of } \cup]} \\
x \in u^{-1} L \cup v^{-1} L . &
\end{array}
$$

Therefore, we can prove that $\mathrm{r}(u)$ is $\mathcal{T}$-prime iff $\rho_{\leqslant_{L_{\mathcal{S}}}^{r}}(u)$ is $L_{\mathcal{S}}$-prime w.r.t. $\mathcal{P}$.

$$
\begin{aligned}
& \mathrm{r}(u)=\bigsqcup_{v \in \mathcal{P}, \mathrm{r}(v) \sqsubset \mathrm{r}(u)} \mathrm{r}(v) \Leftrightarrow \quad \text { [By Equation (6.11)] } \\
& \mathrm{r}(u)=\bigsqcup_{v \in \mathcal{P}, v^{-1} L \subseteq_{\mathcal{S}} u^{-1} L} \mathrm{r}(v) \Leftrightarrow \quad \text { [By Equation (6.12)] }
\end{aligned}
$$

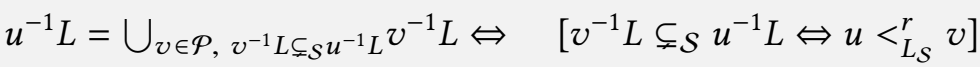

$$
\begin{aligned}
& u^{-1} L=\bigcup_{v \in \mathcal{P}, u<_{L_{\mathcal{S}}}^{r} v^{-1} L} \text {. }
\end{aligned}
$$

It follows from Definitions 6.4.9 (a) and 6.4.4 (a) and Equation (6.12) that $\mathcal{T}$ is closed iff $\leqslant_{L_{S}}^{r}$ is closed.

Moreover, it follows from Definitions 6.4.9 (b) and 6.4.4 (b) that $\mathcal{T}$ is consistent iff $\leqslant_{L_{\mathcal{S}}}^{r}$ is consistent. 
On the other hand, for every $u, v \in \mathcal{P}, a \in \Sigma$ and $x \in \mathcal{S}$ we have that:

$$
\begin{gathered}
(\mathrm{r}(u) \subseteq \mathrm{r}(v)) \wedge(\mathrm{r}(u a)(x)=+) \wedge(\mathrm{r}(v a)(x)=-) \Leftrightarrow \quad \text { [By Equation (6.11)] } \\
\left(u \leqslant_{L_{\mathcal{S}}}^{r} v\right) \wedge(u a x \in L) \wedge(v a x \notin L)
\end{gathered}
$$

It follows that if $\mathcal{T}$ and $\leqslant_{L_{S}}^{r}$ are not consistent then both $\mathrm{NL}^{*}$ and $\mathrm{NL}^{\leqslant}$can find the same word $a x \in \sum \mathcal{S}$ and add it to $\mathcal{S}$. Similarly, it is straightforward to check that if $\mathrm{r}(u a)$ with $u \in \mathcal{P}$ and $a \in \sum$ break consistency, i.e. it is $\mathcal{T}$-prime and it is not equal to any $\mathrm{r}(v)$ with $v \in \mathcal{P}$, then $\rho_{\leqslant_{L_{\mathcal{S}}^{r}}^{r}}(u a)$ is $L_{\mathcal{S}}$-prime for $\mathcal{P}$ and not equal to any $\rho_{\leqslant_{L_{\mathcal{S}}}^{r}}(v)$ with $v \in \mathcal{P}$. Thus, if $\mathcal{T}$ and $\leqslant_{L_{\mathcal{S}}}^{r}$ are not closed then both $\mathrm{NL}^{*}$ and $\mathrm{NL}^{\leqslant}$can find the same word $u a$ and add it to $\mathcal{P}$.

It remains to show that both algorithms build the same automaton modulo isomorphism, i.e., $\mathrm{R}(\mathcal{T})=\langle\widetilde{Q}, \Sigma, \widetilde{(\delta)}, \widetilde{I}, \widetilde{F}\rangle$ is isomorphic to $\mathrm{R}\left(\leqslant_{L_{\mathcal{S}}}^{r}, \mathcal{P}\right)=$ $\langle Q, \Sigma, \delta, I, F\rangle$. Define the mapping $\varphi: Q \rightarrow \widetilde{Q}$ as $\varphi\left(\rho_{\leqslant_{L_{\mathcal{S}}}^{r}}(u)\right)=\mathrm{r}(u)$. Then:

$$
\begin{aligned}
& \varphi(Q)=\left\{\varphi\left(\rho_{\leqslant_{L_{\mathcal{S}}}^{r}}(u)\right) \mid u \in \mathcal{P} \wedge \rho_{\leqslant_{L_{\mathcal{S}}}^{r}}(u) \text { is } L_{\mathcal{S}} \text {-prime w.r.t. } \mathcal{P}\right\} \\
& =\{\mathrm{r}(u) \mid u \in \mathcal{P} \wedge \mathrm{r}(u) \text { is } \mathcal{T} \text {-prime }\}=\widetilde{Q} . \\
& \varphi(I)=\left\{\varphi\left(\rho_{\leqslant_{L_{\mathcal{S}}}^{r}}(u)\right) \mid \varepsilon \in \rho_{\leqslant_{L_{\mathcal{S}}}^{r}}(u)\right\}=\left\{\mathrm{r}(u) \mid u \leqslant_{L_{\mathcal{S}}}^{r} \varepsilon\right\} \\
& =\{\mathrm{r}(u) \mid \mathrm{r}(u) \sqsubseteq \mathrm{r}(\varepsilon)\}=\widetilde{I} . \\
& \varphi(F)=\left\{\varphi\left(\rho_{\leqslant_{\mathcal{S}}^{r}}(u)\right) \mid u \in L \cap \mathcal{P}\right\}=\{\operatorname{r}(u) \mid u \in L \cap \mathcal{P}\} \\
& =\{\mathrm{r}(u) \mid \mathrm{r}(u)(\varepsilon)=+\}=\widetilde{F} . \\
& \varphi\left(\delta\left(\rho_{\leqslant_{L_{\mathcal{S}}}^{r}}(u), a\right)\right)=\varphi\left(\rho_{\leqslant_{L_{\mathcal{S}}}^{r}}(u a)\right)=\left\{\mathrm{r}(v) \mid \rho_{\leqslant_{L_{\mathcal{S}}}^{r}}(u) \in Q \wedge \rho_{\leqslant_{L_{\mathcal{S}}}^{r}}(u) a \subseteq \rho_{\leqslant_{L_{\mathcal{S}}}^{r}}(v)\right\} \\
& =\left\{r(v) \mid r(v) \in \widetilde{Q} \wedge v \leqslant_{L_{\mathcal{S}}}^{r} u a\right\} \\
& =\{\mathrm{r}(v) \mid \mathrm{r}(v) \in \widetilde{Q} \wedge \mathrm{r}(v) \sqsubseteq \mathrm{r}(u a)\} \\
& =\widetilde{\delta}(\mathrm{r}(u), a)=\widetilde{\delta}\left(\varphi\left(\rho_{\leqslant_{L_{\mathcal{S}}}^{r}}(u)\right), a\right) \text {. }
\end{aligned}
$$

Finally, we show that $\varphi$ is an isomorphism. Clearly, the function $\varphi$ is surjective since, for every $u \in \mathcal{P}$, we have that $\mathrm{r}(u)=\varphi\left(\rho_{\leqslant_{L_{\mathcal{S}}}^{r}}(u)\right)$. Moreover $\varphi$ is injective since for every $u, v \in \mathcal{P}, \mathrm{r}(u)=\mathrm{r}(v) \Leftrightarrow u^{-1} L=\mathcal{S} v^{-1} L$, hence $\mathrm{r}(u)=\mathrm{r}(v) \Leftrightarrow \rho_{\leqslant_{L_{\mathcal{S}}}^{r}}(u)=\rho_{\leqslant_{L_{\mathcal{S}}}^{r}}(v)$.

We conclude that $\varphi$ is an NFA isomorphism between $\left.\mathrm{R}\left(\leqslant_{L_{\mathcal{S}}}^{r}, \mathcal{P}\right)\right)$ and $\mathrm{R}(\mathcal{T})$. Therefore $\mathrm{NL}^{*}$ and $\mathrm{NL} \leqslant$ exhibit the same behavior, provided that both algorithms perform the same non-deterministic choices, as they both maintain the same sets $\mathcal{P}$ and $\mathcal{S}$ and build the same automata at each step. 


\section{Termination of $\mathrm{NL}^{*}$ and $\mathrm{NL}^{\leqslant}$}

At each iteration of the $\mathrm{NL}^{\leqslant}$algorithm, it either terminates or the counterexample $w$ given by the Oracle refines the quasiorder $\leqslant_{L_{\mathcal{S}}}^{r}$ which results in having, at least, one new principal $\rho_{\leqslant_{\mathcal{S}}^{r}}^{r}(w)$.

Since

$$
\rho_{\leqslant_{L}^{r}}^{r}(u) \neq \rho_{\leqslant_{L}^{r}}^{r}(v) \Rightarrow \exists s \in \mathcal{S}, u s \in L \wedge v s \notin L \Rightarrow \rho_{\leqslant_{L}^{r}}(u) \neq \rho_{\leqslant_{L}^{r}}(v),
$$

we conclude that the number of principals for $\leqslant_{L_{\mathcal{S}}}^{r}$ is smaller o equal than the number of principals for $\leqslant_{L}^{r}$. Given that $\leqslant_{L}^{r}$ induces finitely many principals, algorithm $\mathrm{NL} \leqslant$ can only add finitely many principals to $\leqslant_{L_{\mathcal{S}}}^{r}$ and, therefore, the algorithm terminates.

It is worth to remark that, in order to prove the termination of the $\mathrm{NL}^{*}$ algorithm, Bollig et al. [2009] first had to show that the number of rows built during the computation of the $\mathrm{NL}^{*}$ algorithm is a lower bound for the number of rows computed during an execution of the $\mathrm{L}^{*}$ algorithm of Angluin [1987]. Then, the termination of the $\mathrm{NL}^{*}$ algorithms follows from the termination of $\mathrm{L}^{*}$.

Finally, observe that, by replacing the right quasiorder $\leqslant_{L_{\mathcal{S}}}^{r}$ by the right congruence $\sim_{L_{\mathcal{S}}} \stackrel{\text { def }}{=} \leqslant_{L_{\mathcal{S}}}^{r} \cap\left(\leqslant_{L_{\mathcal{S}}}^{r}\right)^{-1}$ in the above algorithm (precisely, in Definitions 6.4.9 and 6.4.10), the resulting algorithm corresponds to the $\mathrm{L}^{*}$ algorithm of Angluin [1987]. Note that, in that case, all principals $\rho_{\sim_{L_{\mathcal{S}}}}(u)$, with $u \in \Sigma^{*}$, are $L_{\mathcal{S}}$-prime w.r.t. $\mathcal{P}$. 


\section{FUTURE WORK}

We believe that we have only scratched the surface on the use of well-quasiorders on words for solving problems from Formal Language Theory.

In this section, we present some directions for further developments that show how our work could be extended to (i) take full advantage of simulation relations, (ii) better understand, and possibly improve, the performance of zearch and (iii) develop new algorithms for building smaller residual automata.

\subsection{The Language Inclusion Problem}

Consider the inclusion problem $\mathcal{L}\left(\mathcal{N}_{1}\right) \subseteq \mathcal{L}\left(\mathcal{N}_{2}\right)$, where $\mathcal{N}_{1}$ and $\mathcal{N}_{2}$ are NFAs. Even though we have shown in Chapter 4 that simulations can be used to derive an algorithm for solving this language inclusion problem, we are not on par with the thoughtful use of simulation relations made by Abdulla et al. [2010] and Bonchi and Pous [2013]. The main reason for which we are not able to accommodate within our framework their use of simulations is that our abstraction only manipulates sets of states of $\mathcal{N}_{2}$. As a consequence, any use of simulations that involves states of $\mathcal{N}_{1}$ is out of reach.

However, it is possible to overcome this limitation by using alternating automata as we show next. Intuitively, since alternating automata can be complemented without altering their number of states, we can reduce any language inclusion problem $\mathcal{L}\left(\mathcal{A}_{1}\right) \subseteq \mathcal{L}\left(\mathcal{A}_{2}\right)$, where $\mathcal{A}_{1}$ and $\mathcal{A}_{2}$ are alternating automata, into a universality problem $\Sigma^{*} \subseteq \mathcal{L}\left(\mathcal{A}_{3}\right)$, where $\mathcal{A}_{3}=\mathcal{A}_{1}^{c} \cup \mathcal{A}_{2}$. Since $\mathcal{A}_{3}$ is built by combining the two input automata its states are the union of the states of $\mathcal{A}_{1}$ and $\mathcal{A}_{2}$. Therefore, simulations applicable within our framework to decide $\Sigma^{*} \subseteq \mathcal{L}\left(\mathcal{A}_{3}\right)$, which only involve states of $\mathcal{A}_{3}$, now involve states of $\mathcal{A}_{1}$ and $\mathcal{A}_{2}$. 


\subsubsection{Language Inclusion Through Alternating Automata}

Let $S$ be a set. We denote by $\mathcal{B}^{+}(S)$ the set of positive Boolean formulas over $S$ which are of the form $\Phi \stackrel{\text { def }}{=} s\left|\Phi_{1} \vee \Phi_{2}\right| \Phi_{1} \wedge \Phi_{2} \mid$ false, where $s \in S$ and $\Phi_{1}, \Phi_{2} \in \mathcal{B}^{+}(S)$. We say $S^{\prime} \subseteq S$ satisfies a formula $\Phi \in \mathcal{B}^{+}(S)$ iff $\Phi$ is true when assigning the value true to all elements in $S^{\prime}$ and false to the elements in $S \backslash S^{\prime}$. Given $\Phi \in \mathcal{B}^{+}(S)$, we denote $\llbracket \Phi \rrbracket$ the set of all subsets of $S$ that satisfy $\Phi$. Clearly, if $S^{\prime}$ satisfies a formula $\Phi$, any set $S^{\prime \prime} \subseteq S$ such that $S^{\prime} \subseteq S^{\prime \prime}$ also satisfies $\Phi$. Therefore, the set $\llbracket \Phi \rrbracket$ is an $\subseteq$-upward closed set, i.e. $\rho_{\subseteq}(\llbracket \Phi \rrbracket)=\llbracket \Phi \rrbracket$. Finally, if a formula $\Phi$ is not satisfiable, i.e. no set $S^{\prime} \subseteq S$ satisfies $\Phi$, then $\llbracket \Phi \rrbracket=\varnothing$.

Definition (AFA). An alternating finite-state automata (AFA for short) is a tuple $\mathcal{A} \stackrel{\text { def }}{=}\langle Q, \Sigma, \delta, I, F\rangle$ where $Q$ is the finite set of states, $\Sigma$ is the finite alphabet, $\delta: Q \times \Sigma \rightarrow \mathcal{B}^{+}(Q)$ is the transition function, $I \subseteq Q$ are the initial states and $F \subseteq Q$ are the final states.

Intuitively, given an active state $q \in Q$ and an alphabet symbol $a \in \Sigma$ an AFA can activate any set of states in $\llbracket \delta(q, a) \rrbracket$. Figure 7.1 shows an example of an AFA.

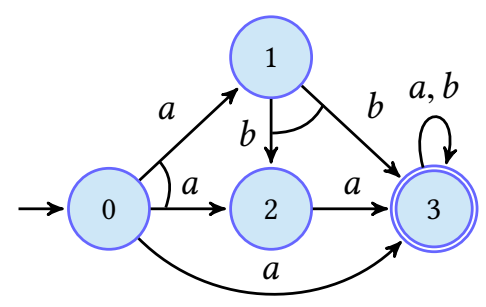

$$
\begin{aligned}
\mathcal{A}=\langle Q, \Sigma, \delta, I, F\rangle \text { with } Q=\left\{q_{0}, q_{1}, q_{2}, q_{3}\right\}, \\
\Sigma=\{a, b\}, I=\left\{q_{0}\right\}, F=\left\{q_{3}\right\} \text { and } \\
\delta\left(q_{0}, a\right)=\left(q_{1} \wedge q_{2}\right) \vee q_{3} \\
\delta\left(q_{1}, b\right)=q_{2} \wedge q_{3} \\
\delta\left(q_{2}, a\right)=\delta\left(q_{3}, a\right)=\delta\left(q_{3}, b\right)=q_{3} \\
\delta\left(q_{0}, b\right)=\delta\left(q_{1}, a\right)=\delta\left(q_{2}, b\right)=\text { false }
\end{aligned}
$$

Figure 7.1: Alternating automaton $\mathcal{A}$ generating the language $\mathcal{L}(\mathcal{A})=a(a+b)^{*}$.

Given an AFA $\mathcal{A}=\langle Q, \Sigma, \delta, I, F\rangle$, we extend the transition function $\delta$ to sets of states obtaining $\Delta: \wp(Q) \times \Sigma \rightarrow \mathcal{B}^{+}$defined as $\Delta(S, a) \stackrel{\text { def }}{=} \bigwedge_{s \in S} \delta(s, a)$. Intuitively, $\Delta(S, a)$ indicates the states that will be activated after reading $a$ when all states in $S$ are active. Let $X \uplus Y \stackrel{\text { def }}{=}\{x \cup y \mid x \in X, y \in Y\}$. Then

$$
\llbracket \Delta(S, a) \rrbracket= \begin{cases}\varnothing & \text { if } \exists s \in S \text { s.t. } \delta(s, a)=\text { false } \\ \biguplus_{s \in S} \llbracket \delta(s, a) \rrbracket & \text { otherwise }\end{cases}
$$

We say a word $w$ is accepted by an AFA $\mathcal{A}=\langle Q, \Sigma, \delta, I, F\rangle$ iff there exists a sequence of sets of active states $S_{0}, \ldots, S_{|w|}$ such that $S_{0}=\left\{q_{i}\right\}$ with $q_{i} \in I$, $S_{n} \subseteq F, S_{n} \neq \varnothing$ and $S_{i} \in \llbracket \Delta\left(S_{i-1},(w)_{i}\right) \rrbracket$ for $1 \leq i \leq|w|$. 
Example 7.1.1. Let us consider the alternating automaton $\mathcal{A}$ in Figure 7.1. In this case, we have that

$$
\begin{aligned}
\Delta\left(\left\{q_{0}\right\}, a\right) & =\delta\left(q_{0}, a\right)=\left(q_{1} \wedge q_{2}\right) \vee q_{3} . \\
\llbracket \Delta\left(\left\{q_{0}\right\}, a\right) \rrbracket & =\llbracket \delta\left(q_{0}, a\right) \rrbracket=\rho_{\subseteq}\left(\left\{\left\{q_{1}, q_{2}\right\},\left\{q_{3}\right\}\right\}\right) . \\
\Delta\left(\left\{q_{1}, q_{2}\right\}, b\right) & =\delta\left(q_{1}, b\right) \wedge \delta\left(q_{2}, b\right)=q_{2} \wedge q_{3} \wedge \text { false }=\text { false } \\
\llbracket \Delta\left(\left\{q_{1}, q_{2}\right\}, b\right) \rrbracket & =\llbracket \delta\left(q_{1}, b\right) \rrbracket \biguplus \llbracket \delta\left(q_{2}, b\right) \rrbracket=\rho_{\subseteq}\left(\left\{\left\{q_{2}, q_{3}\right\}\right\}\right) \uplus \varnothing=\varnothing . \\
\Delta\left(\left\{q_{1}, q_{2}\right\}, a\right) & =\delta\left(q_{1}, a\right) \wedge \delta\left(q_{2}, a\right)=\text { false } \wedge q_{3}=\text { false } \\
\llbracket \Delta\left(\left\{q_{1}, q_{2}\right\}, a\right) \rrbracket & =\llbracket \delta\left(q_{1}, a\right) \rrbracket \biguplus \llbracket \delta\left(q_{2}, a\right) \rrbracket=\varnothing \uplus \rho_{\subseteq}\left(\left\{\left\{q_{3}\right\}\right\}\right)=\varnothing . \\
\Delta\left(\left\{q_{3}\right\}, a\right) & =\Delta\left(\left\{q_{3}\right\}, b\right)=q_{3} \\
\llbracket \Delta\left(\left\{q_{3}\right\}, a\right) \rrbracket & =\llbracket \Delta\left(\left\{q_{3}\right\}, b\right) \rrbracket=\rho_{\subseteq}\left(\left\{\left\{q_{3}\right\}\right\}\right) .
\end{aligned}
$$

Since $q_{0}$ is the only initial state and $F=\left\{q_{3}\right\}$, it follows that the language generated by the automaton is $\mathcal{L}(\mathcal{A})=a(a+b)^{*}$.

We denote the reflexo-transitive closure of $\llbracket \Delta \rrbracket$ as $\leadsto$. Thus, the language of an AFA, $\mathcal{A}$, is $\mathcal{L}(\mathcal{A})=\left\{w \in \Sigma^{*} \mid \exists q_{i} \in I, S \subseteq F, S \neq \varnothing \wedge\left\{q_{i}\right\} \stackrel{w}{\sim} S\right\}$.

One of the most interesting properties of AFAs is that their complement, i.e. an AFA generating the complement language, can be built in polynomial time.

Definition 7.1.2 (Complement of an AFA). Let $\mathcal{A}=\langle Q, \Sigma, \delta, I, F\rangle$ be an AFA with $L=\mathcal{L}(\mathcal{A})$. Its complement AFA, denoted $\mathcal{A}^{c}$ is the $A F A \mathcal{A}^{c} \stackrel{\text { def }}{=}\left\langle Q, \Sigma, \delta^{c}, I, Q \backslash F\right\rangle$ where $\delta^{c}(q, a)$ is the result of switching $\wedge$ and $\vee$ operators in $\delta(q, a)$.

The simplicity of the computation of the complement for AFAs, which does not alter the number of states of the automaton, allows us to use them in order to solve the language inclusion problem $\mathcal{L}\left(\mathcal{N}_{1}\right) \subseteq \mathcal{L}\left(\mathcal{N}_{2}\right)$, where $\mathcal{N}_{1}$ and $\mathcal{N}_{2}$ are NFAs, by reducing it to universality of alternating automata as follows:

$$
\begin{aligned}
\mathcal{L}\left(\mathcal{N}_{1}\right) \subseteq \mathcal{L}\left(\mathcal{N}_{2}\right) \Leftrightarrow & {[\text { Since NFAs } \subseteq \text { AFAs }] } \\
\mathcal{L}\left(\mathcal{A}_{1}\right) \subseteq \mathcal{L}\left(\mathcal{A}_{2}\right) \Leftrightarrow & {\left[A \subseteq B \Leftrightarrow A \cap B^{c}=\varnothing\right] } \\
\mathcal{L}\left(\mathcal{A}_{1}\right) \cap\left(\mathcal{L}\left(\mathcal{A}_{2}\right)\right)^{c}=\varnothing \Leftrightarrow & {\left[(A \cap B)^{c}=A^{c} \cup B^{c} \text { and } \varnothing^{c}=\Sigma^{*}\right] } \\
\left(\mathcal{L}\left(\mathcal{A}_{1}\right)\right)^{c} \cup \mathcal{L}\left(\mathcal{A}_{2}\right)=\Sigma^{*} \Leftrightarrow & {[\text { AFAs are closed under complement] }} \\
\mathcal{L}\left(\mathcal{A}_{1}^{c}\right) \cup \mathcal{L}\left(\mathcal{A}_{2}\right)=\Sigma^{*} \Leftrightarrow & {\left[A=\Sigma^{*} \Leftrightarrow \Sigma^{*} \subseteq A\right] } \\
\Sigma^{*} \subseteq \mathcal{L}\left(\mathcal{A}_{1}^{c}\right) \cup \mathcal{L}\left(\mathcal{A}_{2}\right) \Leftrightarrow & {\left[\text { With } \mathcal{A}_{3}=\mathcal{A}_{1}^{c} \cup \mathcal{A}_{2}\right] } \\
\Sigma^{*} \subseteq \mathcal{L}\left(\mathcal{A}_{3}\right) &
\end{aligned}
$$


On the other hand, $\Sigma^{*}$ is the lfp of the equation $\lambda X .\{\varepsilon\} \cup \cup_{a \in \Sigma} a X$. Therefore

$$
\Sigma^{*} \subseteq \mathcal{L}\left(\mathcal{A}_{3}\right) \Leftrightarrow \operatorname{lfp}\left(\lambda X .\{\varepsilon\} \cup \bigcup_{a \in \Sigma} a X\right) \subseteq \mathcal{L}\left(\mathcal{A}_{3}\right)
$$

We are now in position to leverage our quasiorder-based framework from Chapter 4 to derive an algorithm for deciding the universality of a regular language given by an AFA $\mathcal{A}$. To do that, we adapt our right state-based quasiorder from Equation 4.12, which requires defining the successor operator for AFAs $\operatorname{post}_{w}^{\mathcal{A}}: \wp(\wp(Q)) \rightarrow \wp(\wp(Q))$, where $w \in \Sigma^{*}$, as follows:

$$
\operatorname{post}_{w}^{\mathcal{A}}(X) \stackrel{\text { def }}{=}\left\{S^{\prime} \in \wp(Q) \mid \exists S \in X, S \stackrel{w}{\rightarrow} S^{\prime}\right\} .
$$

It is straightforward to check that post ${ }_{w a}^{\mathcal{A}}(X)=\operatorname{post}_{a}^{\mathcal{A}}\left(\operatorname{post}_{w}^{\mathcal{A}}(X)\right)$. The following example illustrates the behavior of the function $\operatorname{post}_{w}^{\mathcal{A}}$ on the AFA from Figure 7.1.

Example 7.1.3. Consider again the AFA $\mathcal{A}$ from Figure 7.1. We have that

$$
\begin{aligned}
& \operatorname{post}_{a}^{\mathcal{A}}\left(\left\{\left\{q_{0}\right\}\right\}\right)=\rho_{\subseteq}\left(\left\{\left\{q_{1}, q_{2}\right\},\left\{q_{3}\right\}\right\}\right) \\
& \operatorname{post}_{a a}^{\mathcal{A}}\left(\left\{\left\{q_{0}\right\}\right\}\right)=\operatorname{post}_{a}^{\mathcal{A}}\left(\left\{\left\{q_{1}, q_{2}\right\},\left\{q_{3}\right\}\right\}\right)=\rho_{\subseteq}\left(\left\{\left\{q_{3}\right\}\right\}\right) \\
& \operatorname{post}_{a b}^{\mathcal{A}}\left(\left\{\left\{q_{0}\right\}\right\}\right)=\operatorname{post}_{b}^{\mathcal{A}}\left(\left\{\left\{q_{1}, q_{2}\right\},\left\{q_{3}\right\}\right\}\right)=\rho_{\subseteq}\left(\left\{\left\{q_{3}\right\}\right\}\right) .
\end{aligned}
$$

Similarly to what we did in Section 4.3.3 for NFAs, we next define a satebased quasiorder for AFAs, $\leqslant \mathcal{A}$. To do that, let $I_{\{\}}$be the set of singleton subsets of $I$, i.e. $I_{\{\}} \stackrel{\text { def }}{=}\{\{q\} \mid q \in I\}$. Then

$$
u \leqslant \mathcal{A} v \Leftrightarrow \operatorname{post}_{u}^{\mathcal{A}}\left(I_{\{\}}\right) \subseteq \operatorname{post}_{v}^{\mathcal{A}}\left(I_{\{\}}\right)
$$

LEмMA 7.1.4. Let $\mathcal{A}=\langle Q, \Sigma, \delta, I, F\rangle$ be an $A F A$ with $L=\mathcal{L}(\mathcal{A})$. Then $\leqslant \mathcal{A}$ is a right L-consistent well-quasiorder.

Proof. First, we show that $\leqslant_{\mathcal{A}}$ is right monotone. Let $u, v \in \Sigma^{*}$ and $a \in \sum$. Recall that $\operatorname{post}_{a}^{\mathcal{A}}$ is a monotonic function and that

$$
\operatorname{post}_{u v}^{\mathcal{A}}=\operatorname{post}_{v}^{\mathcal{A}} \circ \operatorname{post}_{u}^{\mathcal{A}}
$$

Then

$$
\begin{array}{rlrl}
u \leqslant \mathcal{A} v & \Rightarrow & {[\text { By definition of } \leqslant \mathcal{A}]} \\
\operatorname{post}_{u}^{\mathcal{A}}\left(I_{\{\}}\right) \subseteq \operatorname{post}_{v}^{\mathcal{A}}\left(I_{\{\}}\right) & \Rightarrow & {\left[\text { Since post }{ }_{a}^{\mathcal{A}}\right. \text { is monotonic] }} \\
\operatorname{post}_{a}^{\mathcal{A}}\left(\operatorname{post}_{u}^{\mathcal{A}}\left(I_{\{\}}\right)\right) \subseteq \operatorname{post}_{a}^{\mathcal{A}}\left(\operatorname{post}_{v}^{\mathcal{A}}\left(I_{\{\}}\right)\right) \Leftrightarrow & {[\text { By Equation }(7.5)]} \\
\operatorname{post}_{u a}^{\mathcal{A}}\left(I_{\{\}}\right) \subseteq \operatorname{post}_{v a}^{\mathcal{A}}\left(I_{\{\}}\right) \Leftrightarrow & {[\text { By definition of } \leqslant \mathcal{A}]} \\
u a \leqslant \mathcal{A} v a . & & &
\end{array}
$$


On the other hand, $\leqslant_{\mathcal{A}}$ is $L$-consistent since, by definition

$$
\forall w \in \Sigma^{*}, w \in L \Leftrightarrow \exists S \in \operatorname{post}_{w}^{\mathcal{A}}\left(I_{\{\}}\right), S \neq \varnothing \wedge S \subseteq F .
$$

Therefore, if $u \in L$ and $u \leqslant \mathcal{A} v$ then it follows that $v \in L$.

Finally, it is straightforward to check that $\leqslant \mathcal{A}$ is a well-quasiorder since $\wp(\wp(Q))$ is finite.

Since membership in AFAs is decidable, it follows from Lemma 7.1.4 and Theorem 4.3.4 that Algorithm FAIncWR instantiated with the wqo $\leqslant \mathcal{A}$ decides the inclusion $\Sigma^{*} \subseteq \mathcal{L}(\mathcal{A})$, where $\mathcal{A}$ is an AFA.

Following the developments of Chapter 4, given an AFA $\mathcal{A}=\langle Q, \Sigma, \delta, I, F\rangle$, we could define a Galois Connection $\left\langle\wp\left(\Sigma^{*}\right), \subseteq\right\rangle \stackrel{\gamma}{\stackrel{\gamma}{\leftrightarrows}}\left\langle\mathrm{AC}_{\langle\wp(\wp(Q)), \subseteq\rangle}\right.$, $\rangle$ that yields an antichains algorithm for deciding the universality of AFAs by manipulating sets of sets of states. By doing so, we would obtain an algorithm that computes the set $Y=\left\lfloor\left\{\operatorname{post}_{w}^{\mathcal{A}}\left(I_{\{\}}\right) \mid w \in \Sigma^{*}\right\}\right\rfloor$ and checks whether all elements $y \in Y$ satisfy $\exists s \in y, s \neq \varnothing \wedge s \subseteq F$.

Moreover, we could enhance the state-based quasiorder for AFAs by using simulations between the states of $\mathcal{A}$ which, recall, are the union of the states of the input automata $\mathcal{N}_{1}$ and $\mathcal{N}_{2}$. This would allow us to use the simulations that relate states of both automata, similarly to Abdulla et al. [2010] and Bonchi and Pous [2013].

Therefore, we believe that the full development of an antichains algorithm for AFAs is an interesting line for future work since it will allow us to understand how close our framework can get to the results of Abdulla et al. [2010] and Bonchi and Pous [2013].

\subsection{The Complexity of Searching on Compressed Text}

We believe the good results obtained during the evaluation of zearch (see Figure 5.5) invite for a deeper study of our algorithm in order to better understand its behavior and improve its performance.

For instance, it is yet to be considered how the performance of zearch is affected by the choice of the grammar-based compression algorithm. By using different heuristics to build the grammar, the resulting SLP will have different properties, such as depth, width or length of the rules, which would definitely affect zearch's performance. Figure 7.2 shows the grammars built by different compression algorithms for the same string.

In particular, there are grammar-based compression algorithms such as Sequitur [Nevill-Manning and Witten 1997] that produce SLPs which are not 


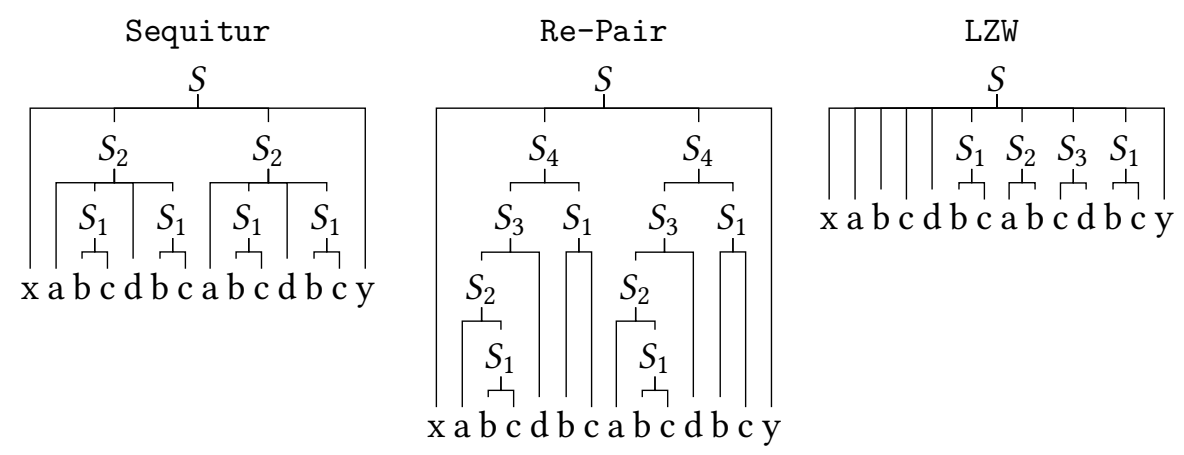

Figure 7.2: From left to right, grammars built by the compression algorithms sequitur [Nevill-Manning and Witten 1997], repair [Larsson and Moffat 1999] and LZW [Welch 1984] for "xabcdbcabcdbcy".

in CNF, i.e. in which rules might have more than two symbols on the right hand side. Processing such a grammar, instead of the one built built by repair reduces the number of rules to be processed at the expense of a greater cost for processing each rule. It is worth considering whether adapting zearch to work on such SLPs will have a positive impact on its performance.

On the other hand, Algorithm CountLines allows for a conceptually simple parallelization since any set of rules such that no variable appearing on the left hand side of a rule appears on the right hand side of another, can be processed simultaneously. Indeed, a theoretical result by Ullman and Gelder [1988] on the parallelization of Datalog queries can be used to show that counting the number of lines in a grammar-compressed text containing a match for a regular expression is in $\mathcal{N} C^{2}$, i.e. it is solvable in polylogarithmic time on parallel computer with a polynomial number of processors, when the automaton built from the expression is acyclic. Therefore, we are optimistic about the possibilities of a parallel version of zearch.

Finally, patterns are a commonly used subclass of regular expressions for which specific searching algorithms have been developed [Kida et al. 1998; Navarro and Tarhio 2005; Gawrychowski 2011; 2014]. Since the standard automata construction from regular expressions yields a DFA when the expression is a pattern, our algorithm allows us to search for patterns in $O(t \cdot s)$ time, where $t$ is the size of the grammar and $s$ is the length of the pattern. However, as shown by Gawrychowski [2011], it is possible to decide the existence of a pattern in an LZW-compressed text in $O(t+s)$ time. It is yet to be considered whether the algorithm of Gawrychowski [2011] can be adapted to the more general scenario of searching on grammar-compressed text and whether it can be extended to re- 
port the number of matching lines without altering its complexity as we did with Algorithm CountLines.

\subsection{The Performance of Residualization}

In Chapter 6 we presented the automata construction $\operatorname{Res}^{r}(\mathcal{N})$ as an alternative to $\mathcal{N}^{\text {res }}$, the residualization operation defined by Denis et al. [2002]. We have shown in Theorem 6.2.6 (e) that given an NFA $\mathcal{N}$, the automaton $\operatorname{Res}^{r}(\mathcal{N})$ is a sub-automaton of $\mathcal{N}^{\text {res }}$, meaning that our construction yields smaller automata.

On the other than, it is clear that, given an NFA $\mathcal{N}=\langle Q, \Sigma, \delta, I, F\rangle$, finding the coverable sets in $\left\{\operatorname{post}_{u}^{\mathcal{N}}(I) \mid u \in \Sigma^{*}\right\}$ is easier than finding the $L$-composite principals in $\left\{\rho_{\leqslant_{N}^{r}}(u) \mid u \in \Sigma^{*}\right\}$. However, it is yet to be considered the performance of both algorithms and the actual difference in size between the RFAs $\operatorname{Res}^{r}(\mathcal{N})$ and $\mathcal{N}^{\text {res }}$.

\subsubsection{Reducing RFAs with Simulations}

Let $\mathcal{N}$ be an NFA with $L=\mathcal{L}(\mathcal{N})$. As shown by Lemma 4.3.9, the simulationbased quasiorder $\leq_{\mathcal{N}}^{r}$ is an $L$-consistent right well-quasiorder. Therefore, it follows from Lemma 6.1.4 that $\mathrm{H}^{r}\left(\leq_{\mathcal{N}}^{r}, L\right)$ is an RFA generating the language $L$. Moreover, as shown in Section 4.3.3.2, we have the following relation between the state-based, the simulation-based and the Nerode's right quasiorders:

$$
\leqslant_{\mathcal{N}}^{r} \subseteq \leq_{\mathcal{N}}^{r} \subseteq \leqslant_{\mathcal{L}(\mathcal{N})}^{r} .
$$

Therefore, by Theorem 6.1.9, we have that

$$
\begin{gathered}
\mid\left\{\rho_{\leqslant_{N}^{r}}(u) \mid u \in \Sigma^{*} \text { and } \rho_{\leqslant_{N}^{r}}(u) \text { is } L \text {-prime }\right\} \mid \\
\mid\left\{\rho_{\leq_{N}^{r}}(u) \mid u \in \Sigma^{*} \text { and } \rho_{\leq_{N}^{r}} \text { is } L \text {-prime }\right\} \mid \\
\mid\left\{\rho_{\leqslant_{L}^{r}}(u) \mid u \in \Sigma^{*} \text { and } \rho_{\leqslant_{L}^{r}}(u) \text { is } L \text {-prime }\right\} \mid .
\end{gathered}
$$

One promising direction for future work is to fully develop this idea of using simulation-based quasiorders to build even smaller RFAs. Such technique should be implemented and evaluated in practice in comparison with the residualization operations $\operatorname{Res}^{r}(\mathcal{N})$ and $\mathcal{N}^{\text {res }}$. 



\section{Conchusions}

In this thesis, we have shown that well-quasiorders are the right tool for addressing different problems from Formal Language Theory. Indeed, we presented two quasiorder-based frameworks in Chapters 4 and 6 that allowed as to offer a new perspective on The Language Inclusion Problem and Residual Automata, respectively. In both cases, our frameworks allowed us to (i) offer a new perspective on known algorithms that facilitates their understanding and evidences the relationships between them and (ii) systematically derive new algorithms, some of which proved to be of practical interest due to their performance.

\section{The Language Inclusion Problem}

We have been able to systematically derive well-known algorithms such as the antichains algorithms for regular languages of Wulf et al. [2006], with its multiple variants (see Section 4.4), and the antichains algorithm for grammars of Holík and Meyer [2015]. These systematic derivations result in a simpler presentation of the antichains algorithm for grammars of Holík and Meyer [2015] as an straightforward extension of the antichains algorithm for regular languages. Indeed, we have shown that the antichains algorithm for regular languages and for grammars are conceptually identical and correspond to two instantiations of our framework with different quasiorders. Recall that, previously, the use of antichains for grammars was justified through a reduction to data flow analysis.

Our framework has also allowed us to derive algorithms for deciding the inclusion of a regular language in the trace set of a one-counter net. In doing so, we have shown that the right Nerode quasiorder for the trace set of a onecounter net is an undecidable well-quasiorder, thereby closing a conjecture made by de Luca and Varricchio [1994, Section 6]. 
Finally, our quasiorder-based framework also allowed us to derive novel algorithms, such as gfp-based Algorithm FAIncGfp, for deciding the inclusion between regular languages. It is yet to be considered the performance of this algorithm in order to decide whether it is of practical interest.

\section{Searching on Compressed Text}

We then adapted the antichains algorithm for grammars to the problem of searching with regular expressions in grammar compressed text. As a result, we have presented the first algorithm for counting the number of lines in a grammarcompressed text containing a match for a regular expression. It is worth to remark that our algorithm applies to any grammar-based compression scheme while being nearly optimal.

Together with the presentation of our algorithm, we described in Chapter 5 the data structures required to achieve nearly optimal complexity for searching in compressed text and used them to implement a sequential tool -zearch- that significantly outperforms the parallel state of the art to solve this problem. Indeed, when the grammar-based compressor achieves hight compression ratio, which is the case, for instance, for automatically generated $\log$ files, zearch uses up to $25 \%$ less time than $1 z 4$ |hyperscan, even outperforming grep and being competitive with hyperscan.

Our results evidence that compression of textual data and regular expression searching, two problems considered independent in practice, are connected. Intuitively, the search can take advantage of the information about repetitions in the text, highlighted by the compressor, to skip parts of the uncompressed text.

\section{Residual Automata}

Denis et al. [2002] introduced the notion of RFA and canonical RFA for a regular language and devised a procedure, similar to the subset construction for DFAs, to build the RFA $\mathcal{N}^{\text {res }}$ from a given automaton $\mathcal{N}$. Furthermore, they showed that the double-reversal method holds for RFAs with their residualization operation, i.e. $\mathcal{N}^{\text {res }}$ is isomorphic to the canonical RFA $C$ for $\mathcal{L}(\mathcal{N})$ for every co-RFA $\mathcal{N}$.

Later, Tamm [2015] proved the following result:

LEMмA 8.1 (Tamm [2015]). Let $\mathcal{N}$ be an NFA and let $C$ be the canonical RFA for $\mathcal{L}(\mathcal{N})$. Then, $\mathcal{N}^{\text {res }}$ is isomorphic to $C$ iff the left language of every state of $\mathcal{N}$ is a union of left languages of states of $C$.

The result of Tamm [2015] generalizes the double-reversal method for RFAs along the lines of the generalization by Brzozowski and Tamm [2014] of the double-reversal method for DFAs which we estate next. 
LeMмA 8.2 (Brzozowski and Tamm [2014]). Let $\mathcal{N}$ be an NFA and let $\mathcal{M}$ be the minimal DFA for $\mathcal{L}(\mathcal{N})$. Then $\mathcal{N}^{D}$ is isomorphic to $\mathcal{M}$ iff the left language of each state of $\mathcal{N}$ is a union of co-atoms of $\mathcal{L}(\mathcal{N})$.

Although the two generalizations have a common foundation, the connection between the two resulting characterizations is not immediate. Our work, together with the work of Ganty et al. [2019] allows us to clarify the relation between these two results and our Theorem 6.3.2. Indeed, Ganty et al. [2019] offered a congruence-based perspective of the generalized double-reversal method for building the minimal DFA which lead to the following result.

LemмA 8.3 (Ganty et al. [2019]). Let $\mathcal{N}$ be an NFA and let $\mathcal{M}$ be the minimal DFA for $\mathcal{L}(\mathcal{N})$. Then $\mathcal{N}^{D}$ is isomorphic to $\mathcal{M}$ iff

$$
\rho_{\sim_{L}^{r}}^{r}\left(W_{I, q}^{\mathcal{N}}\right)=W_{I, q}^{\mathcal{N}},
$$

where $\sim_{L}^{r} \stackrel{\text { def }}{=} \leqslant \frac{r}{=} \cap\left(\leqslant_{L}^{r}\right)^{-1}$ is the right Nerode's congruence.

We believe that the similarity between the generalizations of the doublereversal methods for the minimal DFA (Lemma 8.3) and for the canonical RFA (Theorem 6.3.2), which says that

$$
\operatorname{Res}^{r}(\mathcal{N}) \text { is isomorphic to } C \Leftrightarrow \rho_{\leqslant_{L}^{r}}\left(W_{I, q}^{\mathcal{N}}\right)=W_{I, q}^{\mathcal{N}},
$$

evidences that quasiorders are for RFAs as congruences are for DFAs. Figure 8.1 summarizes the existing results about these double-reversal methods.

Brzozowski and Tamm [2014]

$$
\mathcal{N}^{D} \equiv \mathcal{M}
$$

iff

$\forall q, W_{I, q}^{\mathcal{N}}$ is a union of co-atoms

Ganty et al. [2019]

$$
\mathcal{N}^{\text {res }} \equiv C
$$

$\forall q, W_{I, q}^{\mathcal{N}}$ is a union of $W_{I, q^{\prime}}^{C}$
Theorem 6.3.2

$$
\mathcal{N}^{D} \equiv \mathcal{M}
$$

iff

$\rho_{\sim}^{r}\left(W_{I, q}^{\mathcal{N}}\right)=W_{I, q}^{\mathcal{N}}$
Tamm [2015]

$$
\operatorname{Res}^{r}(\mathcal{N}) \equiv C
$$

$$
\begin{gathered}
\text { iff } \\
\rho_{\leqslant_{L}^{r}}\left(W_{I, q}^{\mathcal{N}}\right)=W_{I, q}^{\mathcal{N}}
\end{gathered}
$$

In the diagram: $\mathcal{N}$ is an NFA with $L=\mathcal{L}(\mathcal{N})$; $\mathcal{N}^{D}$ is the result of determinizing $\mathcal{N}$ with the standard subset construction; $\mathcal{M}$ is the minimal DFA for $L ; C=\operatorname{Can}^{r}(L)$ is the canonical RFA for $L$ and $\mathcal{N}_{1} \equiv \mathcal{N}_{2}$ denotes that automaton $\mathcal{N}_{1}$ is isomorphic to $\mathcal{N}_{2}$.

Figure 8.1: Summary of the existing results about the generalized double-reversal method for building the minimal DFA (first row) and the canonical RFA (second row) for a given language. The results on the first column are based on the notion of atoms of a language while the results on the second column are based on quasiorders. 
Moreover, as shown by Lemma 6.2.8, our residualization operation $\operatorname{Res}^{r}(\mathcal{N})$ offers a desirable property that $\mathcal{N}^{\text {res }}$ lacks: residualizing $\mathcal{N}$ yields the canonical RFA for $\mathcal{L}(\mathcal{N})$ iff $\leqslant_{L}^{r}=\leqslant_{\mathcal{N}}^{r}$. Again, this property is equivalent to the one presented by Ganty et al. [2019] for DFAs.

LEMMA 8.4 (Ganty et al. [2019]). Let $\mathcal{N}$ be an NFA and let $\mathcal{M}$ be the minimal DFA for $\mathcal{L}(\mathcal{N})$. Then $\mathcal{N}^{D}$ is isomorphic to $\mathcal{M}$ iff $\sim_{\mathcal{N}}^{r}=\sim_{L}^{r}$, where $\sim_{\mathcal{N}}^{r} \stackrel{\text { def }}{=} \leqslant_{\mathcal{N}}^{r} \cap\left(\leqslant_{\mathcal{N}}^{r}\right)^{-1}$ is the right state-based congruence.

On the other hand, since Ganty et al. [2019] showed that the left languages of the minimal DFA for a regular language are the blocks of the partition $\rho_{\sim_{L}^{r}}^{r}$, Lemma 8.3 can be equivalently stated as follows.

LEMмA 8.5 (Ganty et al. [2019]). Let $\mathcal{N}$ be an NFA and let $\mathcal{M}$ be the minimal DFA for $\mathcal{L}(\mathcal{N})$. Then $\mathcal{N}^{D}$ is isomorphic to $\mathcal{M}$ iff the left language of each state of $\mathcal{N}$ is a union of left languages of states of the minimal DFA.

Therefore, Lemma 8.5 can be seen as the DFA-equivalent of Tamm's condition for RFAs (Lemma 8.1). Therefore, Lemma 8.5 together with Lemma 8.3, evidence the connection between the generalization of the double reversal for RFAs of Tamm [2015] and the one for DFAs of Brzozowski and Tamm [2014].

Finally, we further support the idea that quasiorders are natural to residual automata by observing that the $\mathrm{NL}^{*}$ algorithm proposed by Bollig et al. [2009] for learning RFAs can be interpreted within our framework as an algorithm that, at each step, refines an approximation of the Nerode's quasiorder and builds an RFA using our automata construction. 


\section{Funding ACKNOWLEDgments}

This research was partially supported by:

1. The Spanish Ministry of Economy and Competitiveness project No. PGC2018102210-B-I00.

2. The Spanish Ministry of Science and Innovation project No. TIN201571819-P.

3. The Madrid Regional Government project No. S2018/TCS-4339 .

4. The Madrid Regional Government project No. S2013/ICE-2731

5. The Ramón y Cajal fellowship RYC-2016-20281.

6. German Academic Exchange Service (DAAD) program "Research Grants Short-Term Grants 2018 (57378443)". 



\section{BibLIOGRAPHY}

Abboud, A., A. Backurs, K. Bringmann, and M. Künnemann

2017. Fine-grained complexity of analyzing compressed data: Quantifying improvements over decompress-and-solve. In 58th IEEE Annual Symposium on Foundations of Computer Science, FOCS 2017, Berkeley, CA, USA, October 15-17, 2017, C. Umans, ed., Pp. 192-203. IEEE Computer Society.

Abdulla, P. A.

2012. Regular model checking. Int. J. Softw. Tools Technol. Transf., 14(2):109118.

Abdulla, P. A., K. Cerans, B. Jonsson, and Y. Tsay

1996. General decidability theorems for infinite-state systems. In Proceedings, 11th Annual IEEE Symposium on Logic in Computer Science, New Brunswick, New Jersey, USA, July 27-30, 1996, Pp. 313-321. IEEE Computer Society.

Abdulla, P. A., Y. Chen, L. Holík, R. Mayr, and T. Vojnar

2010. When simulation meets antichains. In Tools and Algorithms for the Construction and Analysis of Systems, 16th International Conference, TACAS 2010, Held as Part of the foint European Conferences on Theory and Practice of Software, ETAPS 2010, Paphos, Cyprus, March 20-28, 2010. Proceedings, J. Esparza and R. Majumdar, eds., volume 6015 of Lecture Notes in Computer Science, Pp. 158-174. Springer.

Adámek, J., F. Bonchi, M. Hülsbusch, B. König, S. Milius, and A. Silva 2012. A coalgebraic perspective on minimization and determinization. In 
Foundations of Software Science and Computational Structures - 15th International Conference, FOSSACS 2012, Held as Part of the European foint Conferences on Theory and Practice of Software, ETAPS 2012, Tallinn, Estonia, March 24 - April 1, 2012. Proceedings, L. Birkedal, ed., volume 7213 of Lecture Notes in Computer Science, Pp. 58-73. Springer.

Allouche, J.-P., J. Shallit, et al.

2003. Automatic sequences: theory, applications, generalizations. Cambridge university press.

Angluin, D.

1987. Learning regular sets from queries and counterexamples. Inf. Comput., 75(2):87-106.

Antimirov, V. M.

1995. Rewriting regular inequalities (extended abstract). In Fundamentals of Computation Theory, 10th International Symposium, FCT '95, Dresden, Germany, August 22-25, 1995, Proceedings, H. Reichel, ed., volume 965 of Lecture Notes in Computer Science, Pp. 116-125. Springer.

Backurs, A. and P. Indyk 2016. Which regular expression patterns are hard to match? In IEEE 57th Annual Symposium on Foundations of Computer Science, FOCS 2016, 9-11 October 2016, Hyatt Regency, New Brunswick, New Jersey, USA, I. Dinur, ed., Pp. 457466. IEEE Computer Society.

Bille, P., P. H. Cording, and I. L. Gørtz 2014. Compressed subsequence matching and packed tree coloring. In Combinatorial Pattern Matching - 25th Annual Symposium, CPM 2014, Moscow, Russia, June 16-18, 2014. Proceedings, A. S. Kulikov, S. O. Kuznetsov, and P. A. Pevzner, eds., volume 8486 of Lecture Notes in Computer Science, Pp. 40-49. Springer.

Bille, P., R. Fagerberg, and I. L. Gørtz 2009. Improved approximate string matching and regular expression matching on ziv-lempel compressed texts. ACM Trans. Algorithms, 6(1):3:1-3:14.

Bollig, B., P. Habermehl, C. Kern, and M. Leucker 2009. Angluin-style learning of NFA. In IFCAI 2009, Proceedings of the 21st International foint Conference on Artificial Intelligence, Pasadena, California, USA, fuly 11-17, 2009, C. Boutilier, ed., Pp. 1004-1009. 
Bonchi, F. and D. Pous

2013. Checking NFA equivalence with bisimulations up to congruence. In The 40th Annual ACM SIGPLAN-SIGACT Symposium on Principles of Programming Languages, POPL '13, Rome, Italy - January 23 - 25, 2013, R. Giacobazzi and R. Cousot, eds., Pp. 457-468. ACM.

Bringmann, K. and M. Künnemann

2015. Quadratic conditional lower bounds for string problems and dynamic time warping. In IEEE 56th Annual Symposium on Foundations of Computer Science, FOCS 2015, Berkeley, CA, USA, 17-20 October, 2015, V. Guruswami, ed., Pp. 79-97. IEEE Computer Society.

Brzozowski, J. A.

1962. Canonical regular expressions and minimal state graphs for definite events. Mathematical Theory of Automata, 12(6):529-561.

Brzozowski, J. A. and H. Tamm

2014. Theory of átomata. Theor. Comput. Sci., 539:13-27.

Büchi, J. R.

1989. Finite Automata, their Algebras and Grammars - Towards a Theory of Formal Expressions. Springer.

Charikar, M., E. Lehman, D. Liu, R. Panigrahy, M. Prabhakaran, A. Sahai, and A. Shelat

2005. The smallest grammar problem. IEEE Trans. Inf. Theory, 51(7):2554-2576.

Chomsky, N.

1959. On certain formal properties of grammars. Information and Control, 2(2):137-167.

Clarke, E. M., T. A. Henzinger, H. Veith, and R. Bloem 2018. Handbook of Model Checking, 1st edition. Springer Publishing Company, Incorporated.

Cousot, $\mathrm{P}$.

1978. Méthodes itératives de construction et d'approximation de points fixes d'opérateurs monotones sur un treillis, analyse sémantique de programmes (in French). Thèse d'État ès sciences mathématiques, Université Joseph Fourier, Grenoble, France. 


\section{Cousot, P.}

2000. Partial completeness of abstract fixpoint checking. In Abstraction, Reformulation, and Approximation, 4th International Symposium, SARA 2000, Horseshoe Bay, Texas, USA, fuly 26-29, 2000, Proceedings, B. Y. Choueiry and T. Walsh, eds., volume 1864 of Lecture Notes in Computer Science, Pp. 1-25. Springer.

Cousot, P. and R. Cousot

1977. Abstract interpretation: A unified lattice model for static analysis of programs by construction or approximation of fixpoints. In Conference Record of the Fourth ACM Symposium on Principles of Programming Languages, Los Angeles, California, USA, January 1977, R. M. Graham, M. A. Harrison, and R. Sethi, eds., Pp. 238-252. ACM.

Cousot, P. and R. Cousot

1979. Systematic design of program analysis frameworks. In Conference Record of the Sixth Annual ACM Symposium on Principles of Programming Languages, San Antonio, Texas, USA, Fanuary 1979, A. V. Aho, S. N. Zilles, and B. K. Rosen, eds., Pp. 269-282. ACM Press.

D'Alessandro, F. and S. Varricchio 2008. Well quasi-orders in formal language theory. In Developments in Language Theory, 12th International Conference, DLT 2008, Kyoto, Japan, September 16-19, 2008. Proceedings, M. Ito and M. Toyama, eds., volume 5257 of Lecture Notes in Computer Science, Pp. 84-95. Springer.

de Luca, A. and S. Varricchio

1994. Well quasi-orders and regular languages. Acta Inf., 31(6):539-557.

de Luca, A. and S. Varricchio

2011. Finiteness and Regularity in Semigroups and Formal Languages, 1st edition. Springer.

de Moura, E. S., G. Navarro, N. Ziviani, and R. A. Baeza-Yates

1998. Direct pattern matching on compressed text. In String Processing and Information Retrieval: A South American Symposium, SPIRE 1998, Santa Cruz de la Sierra Bolivia, September 9-11, 1998, Pp. 90-95. IEEE Computer Society.

Denis, F., A. Lemay, and A. Terlutte

2000. Learning regular languages using non deterministic finite automata. In Grammatical Inference: Algorithms and Applications, 5th International Colloquium, ICGI 2000, Lisbon, Portugal, September 11-13, 2000, Proceedings, A. L. Oliveira, ed., volume 1891 of Lecture Notes in Computer Science, Pp. 39-50. Springer. 
Denis, F., A. Lemay, and A. Terlutte 2001. Residual finite state automata. 2010:144-157.

Denis, F., A. Lemay, and A. Terlutte 2002. Residual finite state automata. Fundam. Inform., 51(4):339-368.

Denis, F., A. Lemay, and A. Terlutte 2004. Learning regular languages using rfsas. Theor. Comput. Sci., 313(2):267294.

Ehrenfeucht, A., D. Haussler, and G. Rozenberg 1983. On regularity of context-free languages. Theor. Comput. Sci., 27:311-332.

Esparza, J., P. Rossmanith, and S. Schwoon 2000. A uniform framework for problems on context-free grammars. Bulletin of the EATCS, 72:169-177.

Fiedor, T., L. Holík, O. Lengál, and T. Vojnar 2015. Nested antichains for WS1S. 9035:658-674.

Finkel, A. and P. Schnoebelen 2001. Well-structured transition systems everywhere! Theor. Comput. Sci., 256(1-2):63-92.

Ganty, P., E. Gutiérrez, and P. Valero 2019. A congruence-based perspective on automata minimization algorithms. In 44th International Symposium on Mathematical Foundations of Computer Science, MFCS 2019, August 26-30, 2019, Aachen, Germany, P. Rossmanith, P. Heggernes, and J. Katoen, eds., volume 138 of LIPIcs, Pp. 77:1-77:14. Schloss Dagstuhl - Leibniz-Zentrum für Informatik.

Gawrychowski, P.

2011. Optimal pattern matching in LZW compressed strings. In Proceedings of the Twenty-Second Annual ACM-SIAM Symposium on Discrete Algorithms, SODA 2011, San Francisco, California, USA, January 23-25, 2011, D. Randall, ed., Pp. 362-372. SIAM.

Gawrychowski, P.

2014. Simple and efficient lzw-compressed multiple pattern matching. F. Discrete Algorithms, 25:34-41.

Giacobazzi, R. and E. Quintarelli

2001. Incompleteness, counterexamples, and refinements in abstract modelchecking. In Static Analysis, 8th International Symposium, SAS 2001, Paris, 
France, fuly 16-18, 2001, Proceedings, P. Cousot, ed., volume 2126 of Lecture Notes in Computer Science, Pp. 356-373. Springer.

Giacobazzi, R., F. Ranzato, and F. Scozzari 2000. Making abstract interpretations complete. f. ACM, 47(2):361-416.

Ginsburg, S. and H. G. Rice 1962. Two families of languages related to ALGOL. f. ACM, 9(3):350-371.

Henriksen, J. G., J. L. Jensen, M. E. Jørgensen, N. Klarlund, R. Paige, T. Rauhe, and A. Sandholm 1995. Mona: Monadic second-order logic in practice. In Tools and Algorithms for Construction and Analysis of Systems, First International Workshop, TACAS '95, Aarhus, Denmark, May 19-20, 1995, Proceedings, E. Brinksma, R. Cleaveland, K. G. Larsen, T. Margaria, and B. Steffen, eds., volume 1019 of Lecture Notes in Computer Science, Pp. 89-110. Springer.

Hofman, P., R. Mayr, and P. Totzke 2013. Decidability of weak simulation on one-counter nets. In 28th Annual ACM/IEEE Symposium on Logic in Computer Science, LICS 2013, New Orleans, LA, USA, fune 25-28, 2013, Pp. 203-212. IEEE Computer Society.

Hofman, P. and P. Totzke

2018. Trace inclusion for one-counter nets revisited. Theor. Comput. Sci., 735:50-63.

Hofmann, M. and W. Chen

2014. Abstract interpretation from büchi automata. In foint Meeting of the Twenty-Third EACSL Annual Conference on Computer Science Logic (CSL) and the Twenty-Ninth Annual ACM/IEEE Symposium on Logic in Computer Science (LICS), CSL-LICS '14, Vienna, Austria, fuly 14 - 18, 2014, T. A. Henzinger and D. Miller, eds., Pp. 51:1-51:10. ACM.

Holík, L. and R. Meyer

2015. Antichains for the verification of recursive programs. In Networked Systems - Third International Conference, NETYS 2015, Agadir, Morocco, May 1315, 2015, Revised Selected Papers, A. Bouajjani and H. Fauconnier, eds., volume 9466 of Lecture Notes in Computer Science, Pp. 322-336. Springer.

Hopcroft, J. E.

1971. An $\mathrm{n} \log \mathrm{n}$ algorithm for minimizing states in a finite automaton. In Theory of machines and computations, Pp. 189-196. Elsevier. 
Hopcroft, J. E., R. Motwani, and J. D. Ullman

2001. Introduction to Automata Theory, Languages, and Computation - (2. ed.), Addison-Wesley Series in Computer Science. Addison-Wesley-Longman.

Hopcroft, J. E. and J. D. Ullman

1979. Introduction to Automata Theory, Languages, and Computation. AddisonWesley Publishing Company.

Hucke, D., M. Lohrey, and C. P. Reh

2016. The smallest grammar problem revisited. In String Processing and Information Retrieval - 23rd International Symposium, SPIRE 2016, Beppu, Japan, October 18-20, 2016, Proceedings, S. Inenaga, K. Sadakane, and T. Sakai, eds., volume 9954 of Lecture Notes in Computer Science, Pp. 35-49.

Jancar, P., J. Esparza, and F. Moller

1999. Petri nets and regular processes. F. Comput. Syst. Sci., 59(3):476-503.

Kärkkäinen, J., G. Navarro, and E. Ukkonen

2003. Approximate string matching on ziv-lempel compressed text. F. Discrete Algorithms, 1(3-4):313-338.

Kasprzik, A.

2011. Inference of residual finite-state tree automata from membership queries and finite positive data. In Developments in Language Theory - 15th International Conference, DLT 2011, Milan, Italy, fuly 19-22, 2011. Proceedings, G. Mauri and A. Leporati, eds., volume 6795 of Lecture Notes in Computer Science, Pp. 476-477. Springer.

Keil, M. and P. Thiemann

2014. Symbolic solving of extended regular expression inequalities. In 34th International Conference on Foundation of Software Technology and Theoretical Computer Science, FSTTCS 2014, December 15-17, 2014, New Delhi, India, V. Raman and S. P. Suresh, eds., volume 29 of LIPIcs, Pp. 175-186. Schloss Dagstuhl - Leibniz-Zentrum für Informatik.

Khoussainov, B. and A. Nerode

2001. Automata Theory and its Applications. Secaucus, NJ, USA: Birkhäuser Boston.

Kida, T., M. Takeda, A. Shinohara, M. Miyazaki, and S. Arikawa 1998. Multiple pattern matching in LZW compressed text. In Data Compression Conference, DCC 1998, Snowbird, Utah, USA, March 30 - April 1, 1998, Pp. 103112. IEEE Computer Society. 
Klarlund, N.

1999. A theory of restrictions for logics and automata. In Computer Aided Verification, 11th International Conference, CAV'99, Trento, Italy, fuly 6-10, 1999, Proceedings, N. Halbwachs and D. A. Peled, eds., volume 1633 of Lecture Notes in Computer Science, Pp. 406-417. Springer.

Kunc, $M$.

2005. Regular solutions of language inequalities and well quasi-orders. Theor. Comput. Sci., 348(2-3):277-293.

Larsson, N. J. and A. Moffat

1999. Offline dictionary-based compression. In Data Compression Conference, DCC 1999, Snowbird, Utah, USA, March 29-31, 1999, Pp. 296-305. IEEE Computer Society.

Lison, P. and J. Tiedemann

2016. Opensubtitles2016: Extracting large parallel corpora from movie and TV subtitles. In Proceedings of the Tenth International Conference on Language Resources and Evaluation LREC 2016, Portorož, Slovenia, May 23-28, 2016, N. Calzolari, K. Choukri, T. Declerck, S. Goggi, M. Grobelnik, B. Maegaard, J. Mariani, H. Mazo, A. Moreno, J. Odijk, and S. Piperidis, eds. European Language Resources Association (ELRA).

Lohrey, M.

2012. Algorithmics on slp-compressed strings: A survey. Groups Complexity Cryptology, 4(2):241-299.

Mäkinen, V. and G. Navarro 2006. Dynamic entropy-compressed sequences and full-text indexes. In Combinatorial Pattern Matching, 17th Annual Symposium, CPM 2006, Barcelona, Spain, fuly 5-7, 2006, Proceedings, M. Lewenstein and G. Valiente, eds., volume 4009 of Lecture Notes in Computer Science, Pp. 306-317. Springer.

Markey, N. and P. Schnoebelen

2004. A ptime-complete matching problem for slp-compressed words. Inf. Process. Lett., 90(1):3-6.

Miné, A.

2017. Tutorial on static inference of numeric invariants by abstract interpretation. Foundations and Trends in Programming Languages, 4(3-4):120-372. 
Moore, E. F.

1956. Gedanken-experiments on sequential machines. Automata studies, 23(1):60-60.

Navarro, G.

2001. A guided tour to approximate string matching. ACM Comput. Surv., 33(1):31-88.

Navarro, G.

2003. Regular expression searching on compressed text. F. Discrete Algorithms, 1(5-6):423-443.

Navarro, G. and J. Tarhio

2005. Lzgrep: a boyer-moore string matching tool for ziv-lempel compressed text. Softw. Pract. Exp., 35(12):1107-1130.

Nevill-Manning, C. G. and I. H. Witten

1997. Compression and explanation using hierarchical grammars. Comput. $\mathcal{F}$., 40(2/3):103-116.

Park, D.

1969. Fixpoint induction and proofs of program properties. Machine Intelligence, 5 .

Plandowski, W. and W. Rytter

1999. Complexity of language recognition problems for compressed words. In Jewels are Forever, Contributions on Theoretical Computer Science in Honor of Arto Salomaa, J. Karhumäki, H. A. Maurer, G. Paun, and G. Rozenberg, eds., Pp. 262-272. Springer.

Ranzato, F.

2013. Complete abstractions everywhere. In Verification, Model Checking, and Abstract Interpretation, 14th International Conference, VMCAI 2013, Rome, Italy, January 20-22, 2013. Proceedings, R. Giacobazzi, J. Berdine, and I. Mastroeni, eds., volume 7737 of Lecture Notes in Computer Science, Pp. 15-26. Springer.

Rytter, W.

2004. Grammar compression, lz-encodings, and string algorithms with implicit input. In Automata, Languages and Programming: 31st International Colloquium, ICALP 2004, Turku, Finland, July 12-16, 2004. Proceedings, J. Díaz, J. Karhumäki, A. Lepistö, and D. Sannella, eds., volume 3142 of Lecture Notes in Computer Science, Pp. 15-27. Springer. 
Sakarovitch, J.

2009. Elements of Automata Theory. Cambridge University Press.

Schaeffer, L.

2013. Deciding properties of automatic sequences. Master's thesis, University of Waterloo.

Schützenberger, M. P.

1963. On context-free languages and push-down automata. Information and Control, 6(3):246-264.

Tamm, H.

2015. Generalization of the double-reversal method of finding a canonical residual finite state automaton. In Descriptional Complexity of Formal Systems - 17th International Workshop, DCFS 2015, Waterloo, ON, Canada, June 25-27, 2015. Proceedings, J. O. Shallit and A. Okhotin, eds., volume 9118 of Lecture Notes in Computer Science, Pp. 268-279. Springer.

Thompson, K.

1968. Programming techniques: Regular expression search algorithm. Communications of the ACM.

To, A. W. and L. Libkin

2008. Recurrent reachability analysis in regular model checking. In Logic for Programming, Artificial Intelligence, and Reasoning, 15th International Conference, LPAR 2008, Doha, Qatar, November 22-27, 2008. Proceedings, I. Cervesato, H. Veith, and A. Voronkov, eds., volume 5330 of Lecture Notes in Computer Science, Pp. 198-213. Springer.

Ullman, J. D. and A. V. Gelder

1988. Parallel complexity of logical query programs. Algorithmica, 3:5-42.

Welch, T. A.

1984. A technique for high-performance data compression. IEEE Computer, 17(6):8-19.

Wolper, P. and B. Boigelot 1995. An automata-theoretic approach to presburger arithmetic constraints (extended abstract). In Static Analysis, Second International Symposium, SAS'95, Glasgow, UK, September 25-27, 1995, Proceedings, A. Mycroft, ed., volume 983 of Lecture Notes in Computer Science, Pp. 21-32. Springer. 
Wulf, M. D., L. Doyen, T. A. Henzinger, and J. Raskin 2006. Antichains: A new algorithm for checking universality of finite automata. In Computer Aided Verification, 18th International Conference, CAV 2006, Seattle, WA, USA, August 17-20, 2006, Proceedings, T. Ball and R. B. Jones, eds., volume 4144 of Lecture Notes in Computer Science, Pp. 17-30. Springer.

Ziv, J. and A. Lempel

1977. A universal algorithm for sequential data compression. IEEE Trans. Inf. Theory, 23(3):337-343.

Ziv, J. and A. Lempel

1978. Compression of individual sequences via variable-rate coding. IEEE Trans. Inf. Theory, 24(5):530-536. 



\section{Alphabetical Index}

Symbols

L-Consistent Quasiorder $\quad 48$

$L$-Composite Principal

$L_{\mathcal{S}}$-Composite Principal w.r.t. $\mathcal{P} 140$

$W_{S, T}^{\mathcal{N}}$

$W_{S}^{\mathcal{G}}$

KLEENE

$\operatorname{Post}_{\mathcal{N}_{1}}^{\mathcal{N}_{2}}$

$\operatorname{Pre}_{\mathcal{N}_{1}}^{\mathcal{N}_{2}}$

$\mathcal{N}^{\text {res }}$

$\operatorname{ctx}_{L}$

$\leqslant_{L}^{\ell}$

$\leqslant_{\mathcal{N}}^{\ell}$

$\leqslant{ }_{L}^{r}$

$\leqslant_{\mathcal{N}}^{r}$

$\leqslant_{L}$

$\mathcal{N}^{\text {res }}$

$\mathrm{Can}^{\ell}$

$\mathrm{Can}^{r}$

$\operatorname{Res}^{\ell}$

$\operatorname{Res}^{r}$

$\min _{\leqslant}$

$\operatorname{post}_{w}^{\mathcal{N}}$

pre $_{w}^{\mathcal{N}}$

$\leq_{\mathcal{N}}^{l}$

$\leq_{\mathcal{N}}^{r}$

$\sqsubseteq \leqslant$
A ACC 28

additive $\quad 33$

AFA 146

alphabet $\quad 21$

alternating automata $\quad 146$

Antichain 29

Automata construction $\mathrm{H}^{\ell}\left(\leqslant^{\ell}, L\right) \quad 120$

Automata construction $\mathrm{H}^{r}\left(\leqslant^{r}, L\right) \quad 118$

Automata construction $\mathrm{L}\left(\leqslant_{L_{S}}^{r}, \mathcal{P}\right) \quad 141$

Automata Construction $\mathrm{R}(\mathcal{T}) \quad 139$

axiom 26

B

backward antichains algorithm $\quad 14$

backward complete $\quad 37$

Big-O 34

Big-Omega 34

C

Canonical RFA 26

CFG 26

characterizing word 25

Chomsky Normal Form 27

Closed and Consistent Table $\quad 138$

closed line 97

Closedness w.r.t. $\mathcal{P} \quad 141$

Closure 28 


\begin{tabular}{|c|c|c|c|}
\hline closure & 31 & homogeneous regular expressions & 108 \\
\hline $\mathrm{CNF}$ & 27 & & \\
\hline co-additive & 33 & $\mathbf{J}$ & \\
\hline co-deterministic automaton & 23 & Join Operator & 138 \\
\hline co-DFA & 23 & join-semilattice & 28 \\
\hline co-Res & 136 & & \\
\hline co-residual automaton & 25 & K & \\
\hline co-RFA & 25 & Kleene iterates & 30 \\
\hline coarser abstraction & 32 & & \\
\hline complement & 21 & L & \\
\hline Complement of an AFA & 147 & language accepted & 22 \\
\hline complete lattice & 28 & Learner & 137 \\
\hline Composite and Prime Rows & 138 & Least Upper Bound & 28 \\
\hline configuration of an $\mathrm{OCN}$ & 59 & left language & 23 \\
\hline Consistency w.r.t. $\mathcal{P}$ & 141 & Left quasiorder & 28 \\
\hline consistent RFA & 25 & left-adjoint & 33 \\
\hline Context-Free Grammar & 26 & length & 21 \\
\hline Counting Information & 98 & line & 97 \\
\hline coverable & 25 & lub & 28 \\
\hline Covering Relation & 138 & $\mathbf{M}$ & \\
\hline D & & matching line & 97 \\
\hline DCC & 28 & Minimal DFA & 24 \\
\hline deterministic automaton & 23 & minimal elements & 29 \\
\hline determinization & 24 & Minor & 29 \\
\hline DFA & 23 & $\mathbf{N}$ & \\
\hline directed & 28 & $\mathbf{N}$ & \\
\hline directed-complete partial order & 28 & $\begin{array}{l}\text { Nerode's Quasiorders } \\
\text { NFA }\end{array}$ & $\begin{array}{l}29 \\
22\end{array}$ \\
\hline $\mathbf{E}$ & & non-deterministic automaton & 22 \\
\hline easy searching problem & 108 & O & \\
\hline empty & 23 & One-Counter Net & 58 \\
\hline $\mathbf{F}$ & & Oracle & 138 \\
\hline factor & 21 & Orthogonal Vector Problem & 108 \\
\hline forward antichains algorithm & 15 & OVP & 108 \\
\hline G & & $\mathbf{P}$ & \\
\hline Galois Connection & 32 & partially ordered set & 28 \\
\hline glb & 28 & positive Boolean formulas & 146 \\
\hline grammar & 26 & Principal & 28 \\
\hline Greatest Lower Bound & 28 & Q & \\
\hline $\mathbf{H}$ & & quasiorder & 27 \\
\hline hard searching problem & 109 & quasiordered set & 27 \\
\hline homogeneous expression & 109 & Quotient & 21 \\
\hline
\end{tabular}


composite, 22

prime, 22

$\mathbf{R}$

residual automaton

Residualization

residuals

Rest

reverse

Reverse Automaton

RFA

right language

Right quasiorder

right-adjoint

Right-language-based quasiorder w.r.t.

$\mathcal{S}$

140

S

simulation

SLP

25

136

21

23

25

23

28

33,86

56

27
Small-O

34

Straight Line Program 27

string

21

strongly consistent RFA

25

Sub-automaton

23

Subset Construction

24
T

table

Teacher

trace set

138

138

59

U

unreachable 23

Upper Closure Operator $\quad 31$

W

Well-quasiorder

29

word 